\title{
Aerodynamic Characteristics of Atmospheric Boundary Layers
}

\author{
Erich J.Plate

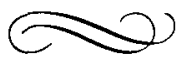 \\ Argonne National Laboratory \\ Karlsruhe University \\ Argonne, Illinois \\ Karlsruhe, West Germany
}

This report was prepared as an account of work sponsored by the United States Government. Neither the United States nor the United States Atomic Energy Commission, nor any of their employees, nor any of their contractors, subcontractors, or their employees, makes any warranty, express or implied, or assumes any legal liability or responsibility for the accuracy, completeness of usefulness of any information, apparatus, product ar process disclosed, or represents that its use would not infringe privately owned rights. 


\section{DISCLAIMER}

This report was prepared as an account of work sponsored by an agency of the United States Government. Neither the United States Government nor any agency Thereof, nor any of their employees, makes any warranty, express or implied, or assumes any legal liability or responsibility for the accuracy, completeness, or usefulness of any information, apparatus, product, or process disclosed, or represents that its use would not infringe privately owned rights. Reference herein to any specific commercial product, process, or service by trade name, trademark, manufacturer, or otherwise does not necessarily constitute or imply its endorsement, recommendation, or favoring by the United States Government or any agency thereof. The views and opinions of authors expressed herein do not necessarily state or reflect those of the United States Government or any agency thereof. 


\section{DISCLAIMER}

Portions of this document may be illegible in electronic image products. Images are produced from the best available original document. 
Available as TID-25465 for $\$ 3.00$ from

National Technical Information Service

U.S. Department of Commerce

Springfield, Virginia 22151

Library of Congress Catalog Card Number: 70-611329

Printed in the United States of America

USAEC Division of Technical Information Extension, Oak Ridge, Tennessee

May 1971 


\section{PREFACE}

The planetary or atmospheric boundary layer is that region of the atmospneric surface layer which is directly affected by the friction between the ground and the atmosphere. Since the atmospheric air is a real fluid, the velocity gradients transmit the surface shear from the ground to higher elevations, up to a certain height $h$ called the thickness of the atmospheric boundary layer, above which ground effects are no longer important. This thickness $h$ varies with local terrain conditions and is typically of the order of 100 to $1000 \mathrm{~m}$. It is many times smaller than typical horizontal length scales of the atmosphere, and we can consider the flow in the surface layer to be of boundary-layer nature, i.e., vertical velocities are small everywhere as compared with velocities parallel to the ground.

Under these conditions the air flow of the planetary boundary layer becomes uncoupled from the air flow aloft, i.e., the planetary boundary layer is affected by the outer atmosphere but not vice versa. Strictly speaking, this is of course not true because the atmospheric motions are strongly affected by the ground and in particular by orographic terrain features of large magnitude. Locally, however, and over a terrain that at most exhibits small-scale configuration changes, the planetary boundary layer can be considered as shaped by the boundary conditions impressed by the noninteracting outer atmosphere and by the lower boundary conditions set by the terrain characteristics.

In the planetary boundary layer, air motions are induced by the pressure gradients imposed by large-scale atmospheric pressure fields and by the diurnal heating cycle set up by solar radiation. The resulting temperature and velocity fields represent the natural conditions of the atmosphere in which most human activities take place. Man-made modifications of the environment affect and are affected by the planetary 
boundary layer, and in some areas these interactions have become strong enough to make it desirable that they be understood and predicted Because of recent developments in techniques for constructing high-rise buldings, the structural engineer should know the maximum wind conditions with some accuracy Agricultural meteorologists wish to make better predictions for erosion protection of solls, or of evaporation from lakes and irrigated surfaces Urban authorities would like to have avallable techniques by which to set standards for air pollution or determine which source of pollution must be eliminated before air quality has deteriorated to an unacceptable level To solve these problems, and many more of a similar type, we need to calculate wind and temperature distributions in the planetary boundary layer, or at least in 1 ts lowest part

In the last few decades, many meteorologists, physicists, and engineers have contributed to such an extent to our understanding of the planetary boundary layer, and in particular of its lowest part, that a reasonably complete physical picture of the flow processes in it is avalable One of the purposes of this report is to summarize rather completely what is known about mean flow conditions in the planetary boundary layer The discussion goes considerably beyond the well-known presentation of Lumley and Panofsky (1964) and Priestley (1959) in the coverage of mean velocity and temperature distributions, whereas the subject of turbulence has only been mentioned when necessary to obtain closures to the equations of motion and energy.

The subject has been developed in four chapters In Chap 1 the two-layer model for the planetary boundary in neutral conditions is developed A consistent formulation for mean velocity distributions in both layers is given which yields the logarithmic law near the ground This law is discussed in some detal because it is fundamental not only for micrometeorological situations but also for modeling of the atmosphere in wind tunnels The chapter contains a discussion of canopy flows and ends with some considerations on modeling

Chapter 2 covers the stratıfied boundary layer near the ground Stablity and its effect on the turbulence structure are discussed, and formulations for mean temperature and velocity distributions are reviewed on the basis of the Monın-Obukhov similarity theory The structure of free-convection layers is treated in Chap 3, from which it becomes clear that in an unstably stratified boundary layer the Ekman-type planetary boundary-layer models are not useful Finally, in Chap 4, disturbed boundary layers are discussed The two problems considered are the two-dimensional boundary layer developing downstream of a crosswind discontinuity in roughness and the flow downwind of a shelterbelt

The purpose of this report goes beyond giving a state-of-the-art review of what is known about the planetary boundary layer It also contains a series of suggestions for future research to extend the limits of our knowledge, with particular emphasis on laboratory experıments For many years I have worked on aerodynamic modeling of the lower part of the atmospheric boundary layer in a wind tunnel, and I feel that its possibilities as a basic tool for research in fundamental problems of the lower atmosphere are far from exhausted Some of the areas which invite wind-tunnel studies are outlined briefly at the end of each chapter 
It is significant that more than $50 \%$ of the references on which this report is based were written in the 1960's During the last decade much of the existing knowledge has been reevaluated and in some cases put into a better light I have made no attempt to be complete in the coverage of the literature Rather, I used references which were of direct help in developing my preferred line of thought, while parallel and perhaps equally valid developments in other papers have either been given less emphasis or no coverage at all

I thank $\mathrm{H}$ Moses and Dr $\mathrm{P}$ Gustafson for giving me the opportunity to write this report while I was a Visitıng Scientist at Argonne National Laboratory Earlier drafts of some of the chapters were reviewed by Drs J Deardorff and J Businger Their comments as well as results of discussions with Drs $\mathrm{H}$ Lettau and P Frenzen are reflected in the report, but of course the choice of material presented and the preferences expressed thereby are solely my own

Ench J Plate

Professor of Civll Engineering

Karlsruhe University

Karlsruhe, West Germany 
-

0 


\section{CONTENTS}

1 The Neutrally Stratified Boundary Layer over Uniform Terrain

Introduction

The Equations of Motion

The Planetary Boundary Layer

Velocity Distribution near the Ground

Modeling the Planetary Boundary Layer

References

\section{The Stratified Atmospheric Boundary Layer} near the Ground

Introduction

Basic Equations for Slightly Stratified Fluids

Turbulent Motions in Stratified Shear Flows to the Boussinesq Approximation

Mean Velocity Distributions in Stratified-

Boundary-Layer Flows

Temperature Profiles and Heat Flux in Stratified Air 
viii

3 Free-Convection Layer $\quad 99$

Introduction $\quad 99$

Boussinesq Equations for Free Convection $\quad 100$

Steady Convection in a Layer Between Two

Parallel and Horizontal Plates 103

Steady Convection in an Infinite-Thickness Layer $\quad 115$

Unsteady Free Convection $\quad 116$

Free Convection Capped by a Stable Layer $\quad 121$

Experiments on the Free-Convection Layer 133

References 133

4 Two-Dimensional Disturbed Boundary Layers 137

Introduction $\quad 137$

The Internal Boundary Layer $\quad 138$

$\begin{array}{ll}\text { Shelterbelts } & 161\end{array}$

Research Needs on Disturbed Boundary Layers $\quad 177$

$\begin{array}{ll}\text { References } & \mathbf{1 7 8}\end{array}$

$\begin{array}{ll}\text { Author Index } & 183\end{array}$

$\begin{array}{ll}\text { Subject Index } & 187\end{array}$ 


\section{THE NEUTRALLY STRATIFIED BOUNDARY LAYER OVER UNIFORM TERRAIN}

\section{INTRODUCTION}

The reference case for all investigations of the structure of the atmospheric boundary layer is the neutrally stratified wind over uniform terrain. The effect of temperature on the wind profile, or the change in profile due to nonuniform terrain, is measured in terms of the deviation that is caused by the actual profile from this reference condition. It is therefore appropriate to devote Chap. 1 to a discussion of this case.

It has recently been recognized, in particular by Blackadar and Tennekes (1968) and Csanady (1967), that the planetary boundary layer consists of two layers, each of which is governed by a different set of flow parameters. This flow structure is quite similar to that in the turbulent boundary layer along a flat plate. The lower layer is very closely analogous to that found in the aerodynamic boundary layer, in both cases resulting in a profile shape that is logarithmic. This analogy is essential for representing the boundary layer of the atmosphere by the boundary layer along a flat plate in a wind tunnel, and a demonstration of the analogy yields a post facto validation of wind-tunnel modeling of mean velocity prufiles for purposes of determining wind loads on structures and similar problems.

A difference between the two situations exists in the outer part of the boundary layer. No direct analogy exists because the planetary boundary layer is driven by both Coriolis and pressure forces. The balance of these forces provides a means of sustaining a motion without changing the momentum of the fluid, i.e., there can exist a constant-thickness turbulent planetary boundary layer with a velocity distribution that depends on the vertical coordinate only. Then a very simple asymptotic equation for 
the shear-stress distribution in the outer part of the boundary layer can be derived, as has recently been discovered by Swinbank (1969), which can be used to obtain the asymptotic form of the velocity distribution near the outer edge of the planetary boundary layer. With this equation we can construct a self-consistent and dimensionally homogeneous set of parameters describing the profiles, by means of a few experiments, which have been reported in the literature. The methods of obtaining the asymptotic profiles are outlined in the first half of this chapter.

The remainder of this chapter covers special forms of the logarithmic law for different surfaces, with some consideration of flow within very large roughness elements, i.e., canopy flow. Discussed last is modeling of the atmospheric boundary layer in a wind tunnel.

\section{THE EQUATIONS OF MOTION}

\section{Momentum Equations for Turbulence Flow}

Throughout this chapter a right-hand coordinate system will be used. This coordinate system is placed on the surface of the earth in such a manner that the z-axis is perpendicular to the gravitational equipotentials, pointing upward, and $x$ - and $y$-axes are in a tangential plane to the earth surface, as shown in Fig. 1.1. In such a coordinate system, the rotation of the earth gives rise to a centrifugal acceleration, which because of the large radius of the earth can be neglected, and to a Coriolis acceleration, $a_{c}$

$$
a_{c}=2 \omega k^{\prime} \times v
$$

where $\mathbf{v}$ is the velocity vector at the location considered, $\omega$ is the rotation of the earth $=2 \pi / 24 \mathrm{hr}=(7.29)\left(10^{-5}\right) \mathrm{sec}^{-1}$, and $\mathbf{k}^{\prime}$ is a unit vector in the direction of the axis of rotation. Thus the Coriolis acceleration is perpendicular both to the axis of rotation and to the plane of the velocity vector, $\mathbf{v}$.

In the contemplated situation of the planetary boundary layer, the vertical velocities are everywhere small and only that portion of the vector $\omega \mathbf{k}^{\prime}$ contributes to the Coriolis acceleration which is perpendicular to the $x-y$ plane, i.e., in the $z$ direction, and whose magnitude is $\omega \sin \lambda$, where $\lambda$ is the geographic latitude. The Coriolis acceleration then becomes

$$
2 \omega \mathbf{k}^{\prime} \times \mathbf{v} \approx 2 \omega \sin \lambda \mathbf{k} \times \mathbf{v}
$$

where $\mathbf{k}$ is the unit vector in the $\mathrm{z}$ direction. It is customary to introduce the Coriolis parameter $f$ defined to

$$
\mathrm{f}=2 \omega \sin \lambda
$$




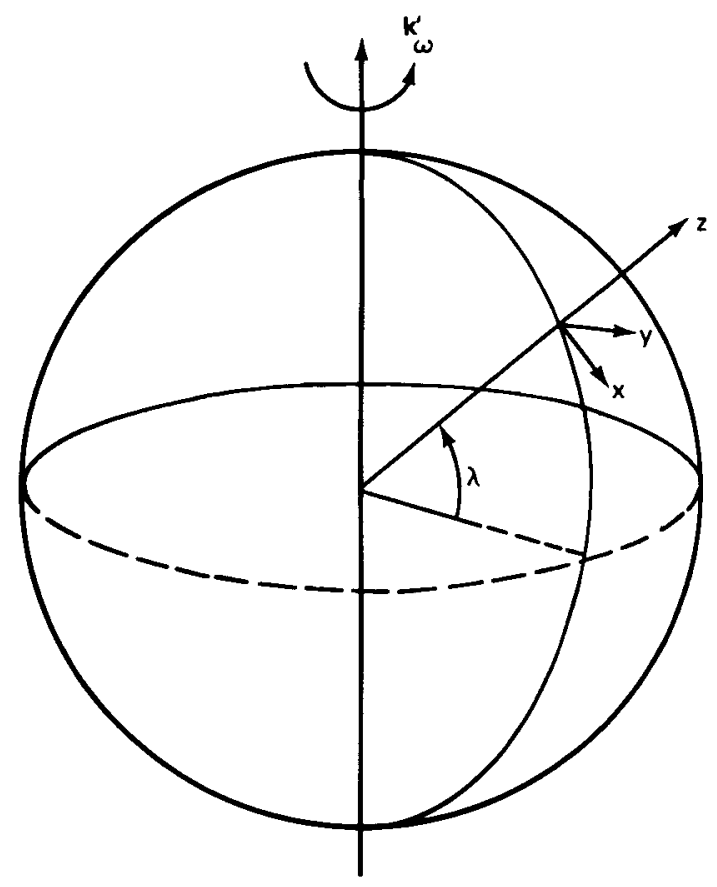

Fig. 1.1 Orientation of a local coordinate system with respect to the rotating earth.

which will be used for expressing the effect of the Coriolis acceleration.

With the Coriolis acceleration the equation of motion for a viscous fluid becomes, in vector notation,

$$
\frac{\partial \mathrm{v}}{\partial \mathrm{t}}+(\mathbf{v} \cdot \nabla) \mathrm{v}+\mathrm{fk} \times \mathrm{v}=-\frac{1}{\rho} \nabla \mathrm{p}-\nu \nabla^{2} \mathbf{v}
$$

and the equation of continuity is

$$
\nabla \cdot \mathbf{v}=0
$$

In these equations, incompressibility of the air has been assumed. The justification for this approximation will be given in Chap. 2 .

Since the flow is turbulent, all quantities appearing in Eqs. 1.4 and 1.5 will be separated into a time-average part, denoted by an overbar, and a fluctuating part, defined by the operation

$$
\begin{gathered}
\bar{u}=\frac{1}{T} \int_{0}^{T} u d t \\
u=\bar{u}+u^{\prime}
\end{gathered}
$$


Therefore the time average of $u^{\prime}$ is equal to zero. During the averaging process the averaging time $\mathrm{T}$ is to some extent arbitrary. Supposedly the time $\mathrm{T}$ is short compared to the time scale of the changes in boundary conditions for the planetary boundary layer but long compared to the decay time of turbulent fluctuations that are generated by the interaction of the turbulent shearing stress with the mean velocity gradient. These time scales will be defined more precisely in the following section of this chapter; here it suffices to point out the ambiguity in defining time averages.

By introducing fluctuating and mean quantities into the equations of motion and continuity and then averaging, we obtain the equations for the mean motion:

$$
\begin{gathered}
\frac{\partial \overline{\mathbf{v}}}{\partial \mathrm{t}}+(\overline{\mathrm{v}} \cdot \nabla) \overline{\mathrm{v}}=-\frac{1}{\rho} \nabla \overline{\mathrm{p}}+\nu \nabla^{2} \overline{\mathbf{v}}-\overline{\left(\mathbf{v}^{\prime} \cdot \nabla\right) \mathrm{v}^{\prime}}-\mathrm{fk} \times \overline{\mathbf{v}} \\
\nabla \cdot \mathrm{v}^{\prime}=0
\end{gathered}
$$

Subtracting these equations from Eqs. 1.4 and 1.5 gives two equations for the turbulent motion:

$$
\frac{\partial \mathbf{v}^{\prime}}{\partial \mathrm{t}}+\left(\mathbf{v}^{\prime} \cdot \nabla\right) \overline{\mathbf{v}}+(\overline{\mathbf{v}} \cdot \nabla) \mathbf{v}^{\prime}=-\frac{1}{\rho} \nabla \mathrm{p}^{\prime}+\nu \nabla^{2} \mathbf{v}^{\prime}-\mathbf{f k} \times \mathbf{v}^{\prime}
$$

and

$$
\nabla \cdot \mathrm{v}^{\prime}=0
$$

These equations have to satisfy the boundary conditions that are imposed on the planetary boundary layer. Above the surface layer the motions are due to synoptic pressure changes that set the outer boundary conditions on the surface layer. An often used set of boundary conditions depicting this situation is obtained from the requirement that at $z=h$ the velocity $u$ be equal to a reference velocity, $u_{\text {ref }}$. For example, the geostrophic velocity $u_{\text {ref }}=G$, to be defined below, or the condition $u_{\text {ref }}=u_{h}$, where $u_{h}$ is the velocity measured at some height $h$. In contrast to the geostrophic wind, the velocity $u_{h}$ cannot be predicted from synoptic information; on the other hand, it is a real quantity that need not be inferred from such relatively crude information as maps of isobars.

The lower boundary conditions for the equations are given by the condition of the ground. The flow in the atmospheric surface layer will reflect topographic and surface influences of the terrain from a large area over which it developed. As shall be discussed in more detail in Chap. 4, a layer of thickness $h$ is affected by an upstream area of at least $10 \mathrm{~h}$ in longitudinal extent, and the vertical structure of the wind field above a point has integrated into it all terrain features of the approach area. A description of the local surface layer above a highly nonuniform terrain is not generally feasible; and most of the significant results have been obtained for flow over uniform terrain. Fortunately such a restriction is not too severe as long as uniformity 
exists on the average over a large area because localized strong gradients in the velocity profiles are rapidly flattened by local mixing of the fluid. If the elements causing the localized gradients are randomly spaced in a uniform pattern, then it can be expected that at some distance above the surface the wind structure is homogenized and can be treated as if the terrain were uniform.

\section{Dimensionless Parameters of the Turbulent Motion}

A discussion of the equations of mean motion in nondimensional form is useful for finding simplifications that facilitate a study of the velocity distributions in the planetary boundary layer. For this purpose let $T_{R}$ be a reference time, $L_{R}$ a reference length, $V_{R}$ a reference velocity, and $P_{R}$ a reference pressure. If nondimensional quantities are denoted by a subscript 1 , then we obtain Eqs. 1.8 and 1.9 in the form

$$
\nabla \cdot \overline{\mathbf{v}}_{1}=0
$$

and

$$
\begin{aligned}
\frac{\mathrm{V}_{\mathrm{R}}}{\mathrm{T}_{\mathrm{R}}} \frac{\partial \overline{\mathbf{v}}_{1}}{\partial \mathrm{t}_{1}}+\frac{\mathrm{V}_{\mathrm{R}}^{2}}{\mathrm{~L}_{\mathrm{R}}}\left(\overline{\mathbf{v}}_{1} \cdot \nabla\right) \overline{\mathrm{v}}_{1}=-\frac{\mathrm{P}_{\mathrm{R}}}{\rho \mathrm{L}_{\mathrm{R}}} \nabla \overline{\mathrm{p}}_{1}+\frac{\nu \mathrm{V}_{\mathrm{R}}}{\mathrm{L}_{\mathrm{R}}^{2}} \nabla^{2} \overline{\mathbf{v}}_{1} \\
\\
\quad-\frac{\mathrm{V}_{\mathrm{R}}^{2}}{\mathrm{~L}_{\mathrm{R}}}\left(\mathrm{v}_{1}^{\prime} \cdot \nabla\right) \mathrm{v}_{1}^{\prime}-\mathrm{fV}_{\mathrm{R}} \mathbf{k} \times \overline{\mathbf{v}}_{1}
\end{aligned}
$$

Dividing by $\mathrm{V}_{\mathrm{R}}^{2} / \mathrm{L}_{\mathrm{R}}$ and dropping subscripts yield

$\frac{\mathrm{L}_{\mathrm{R}}}{\mathrm{V}_{\mathrm{R}} \mathrm{T}_{\mathrm{R}}} \frac{\partial \overline{\mathbf{v}}}{\partial \mathrm{t}}+(\overline{\mathrm{v}} \cdot \nabla) \overline{\mathrm{v}}=-\frac{\mathrm{P}_{\mathrm{R}}}{\rho \mathrm{V}_{\mathrm{R}}^{2}} \nabla \overline{\mathrm{p}}+\frac{\nu}{\mathrm{V}_{\mathrm{R}} \mathrm{L}_{\mathrm{R}}} \nabla^{2} \overline{\mathbf{v}}-\left(\mathbf{v}^{\prime} \cdot \nabla\right) \mathbf{v}^{\prime}-\frac{\mathrm{f} \mathrm{L}_{\mathrm{R}}}{\mathrm{V}_{\mathrm{R}}} \mathbf{k} \times \overline{\mathbf{v}}$

The nondimensional numbers appearing in each of the terms are indicative of the relative magnitudes of the terms as compared to convective acceleration terms. Large numbers imply that the corresponding terms are large when compared with the inertia terms.

A small dimensionless time, $L_{R} / V_{R} T_{R}$, implies that the flow behaves as if it were steady. In the fluid layer near the ground, whose characteristic vertical scale can be set equal to its thickness $h$, the condition of steadiness is satisfied if the boundary conditions change so gradually that a characteristic time reflecting this change, such as $T_{R}=G /(d G / d t)$, is large compared with characteristic times reflecting the boundarylayer adjustment, such as $h / u_{*}$ (very roughly), where $u_{*}$ is the shear velocity defined by

$$
u_{*}=\sqrt{\frac{\tau_{0}}{\rho}}
$$


with $\tau_{0}$ the shear stress at the ground level and $\rho$ the fluid density. The latter time scale is of the order of $1 \mathrm{hr}$, so that a pressure pattern that remains constant for a few hours can be expected to create a steady flow field.

The pressure or Euler number, $\mathrm{P}_{\mathrm{R}} / \rho \mathrm{V}_{\mathrm{R}}^{2}$, usually is of the order 1 or larger because the pressure-force term is the one that drives the velocity field. The parameter determining the order of the viscous term is the Reynolds number

$$
\operatorname{Re}=\frac{V_{R} L_{R}}{\nu}
$$

which in the planetary boundary layer is very large. Thus only when the nondimensional inertia terms are very small compared to $\nabla^{2} \mathrm{v}$ can the viscous term contribute to the momentum balance. This is the case near solid boundaries where the no-slip condition leads to very small velocities and very large velocity gradients. If the flow were laminar, then the viscous term alone would support the momentum flux to the wall in a thin, viscous boundary layer. In the presence of turbulence, the turbulent inertia term has a component that acts in the same manner as the viscous term except that it is much larger near the wall. The result is that, for the same shear stress at the wall, the layer which is affected by the wall shearing stress is thicker in turbulent flow.

The similarity in the action of the turbulent inertia term and the viscous term leads to the inference that the Reynolds number also governs the turbulent inertia term. However, the effect of the Reynolds number here is not as clear as for viscous action. As will be shown in a later chapter, we can often neglect the effect of Reynolds number changes if the Reynolds number is large enough. The Reynolds number must exceed the critical Reynolds number at which transition from laminar to turbulent flow takes place. But the magnitude of the Reynolds number above which a change in the Reynolds number no longer affects the flow pattern is not well established and depends on the geometry of the situation.

The last nondimensional number in Eq. 1.14 is the Rossby number

$$
\mathrm{Ro}_{0}=\frac{\mathrm{fL}_{\mathrm{R}}}{\mathrm{V}_{\mathrm{R}}}
$$

which determines the relative magnitude of the Coriolis acceleration as compared to the convective acceleration. A large Rossby number makes the convective acceleration terms negligible as compared with the other terms, a situation that will be considered in this chapter. The presence of the Coriolis force gives rise to a force that can balance the shear terms and the pressure forces without requiring a momentum change of the fluid. Thus a possible product, under ideal conditions, is a constant-thickness layer, in which the mean velocity depends on $\mathrm{z}$ only. The homogeneity in planes $\mathrm{z}=$ constant requires not only constancy of $\mathbf{v}$ but also of the turbulent mean quantities, and we find that

$$
\overline{\left(v^{\prime} \cdot \nabla\right) v^{\prime}}=\overline{v^{\prime} \frac{\partial w^{\prime}}{\partial z}}
$$


With this expression the equations of motion for the mean flow become, in component form:

$$
\begin{gathered}
0=-\frac{1}{\rho} \frac{\partial \overline{\mathrm{p}}}{\partial \mathrm{x}}+\frac{\partial}{\partial \mathrm{z}}\left(\nu \frac{\partial \overline{\mathrm{u}}}{\partial \mathrm{z}}-\overline{\mathrm{u}^{\prime} \mathrm{w}^{\prime}}\right)+\overline{\mathrm{v} f} \\
0=-\frac{1}{\rho} \frac{\partial \overline{\mathrm{p}}}{\partial \mathrm{y}}+\frac{\partial}{\partial \mathrm{z}}\left(\nu \frac{\partial \overline{\mathrm{v}}}{\partial \mathrm{z}}-\overline{\mathrm{v}^{\prime} \mathrm{w}^{\prime}}\right)-\overline{\mathrm{u} f} \\
0=-\frac{1}{\rho} \frac{\partial \overline{\mathrm{p}}}{\partial \mathrm{z}}-\frac{\partial}{\partial \mathrm{z}} \overline{\left(\mathrm{w}^{\prime 2}\right)}
\end{gathered}
$$

in which the equation of continuity has been used to write

$$
\left(\mathbf{v}^{\prime} \cdot \nabla\right) \mathbf{v}^{\prime}=\left(\nabla \cdot \mathbf{v}^{\prime}\right) \mathbf{v}^{\prime}-\frac{\partial}{\partial z}\left(\mathrm{w}^{\prime} \mathbf{v}^{\prime}\right)
$$

Equation 1.21 can be integrated directly to yield

$$
\bar{p}=\bar{p}(x, y)-\overline{w^{\prime 2}}
$$

and since $\overline{\mathrm{w}^{2}}$ is a function of $\mathrm{z}$ only, the gradient of $\overline{\mathrm{p}}$ in the $\mathrm{x}-\mathrm{y}$ plane is a function of $x$ and $y$ only, i.e., it is the same at the height $h$ as it is at the ground. It is therefore impressed by the outer boundary conditions.

\section{The Energy Equation for the Planetary Boundary Layer}

To Eqs. $1.19,1.20$, and 1.23 we can add the energy equation, which is obtained by dot multiplying Eq. 1.4 by $\mathbf{v}^{\prime}$ and averaging with respect to time. From Eq. 1.10, we obtain the energy balance of the component velocities:

$$
\begin{aligned}
& \mathbf{v}^{\prime} \cdot\left(\mathbf{v}^{\prime} \cdot \nabla\right) \mathbf{v}^{\prime}=-\nabla \cdot\left[\mathbf{v}^{\prime}\left(\mathbf{v}^{\prime} \cdot \mathbf{v}^{\prime}\right)\right]=-\frac{\partial}{\partial \mathrm{z}} \mathrm{w}^{\prime}\left(\mathbf{v}^{\prime} \cdot \mathbf{v}^{\prime}\right) \\
& \frac{1}{2} \frac{\partial \overline{u^{\prime 2}}}{\partial \mathrm{t}}+\overline{u^{\prime} w^{\prime}} \frac{\partial \bar{u}}{\partial z}=-\frac{1}{\rho} \overline{u^{\prime} \frac{\partial p^{\prime}}{\partial x}}-\frac{\partial}{\partial z} \overline{w^{\prime} u^{\prime 2}} \\
& +\frac{1}{2} \nu\left(\frac{\partial^{2} \overline{u^{\prime 2}}}{\partial z^{2}}\right)-v\left[\left(\frac{\partial \overline{u^{\prime}}}{\partial x}\right)^{2}+\left(\frac{\partial \overline{u^{\prime}}}{\partial y}\right)^{2}+\left(\frac{\partial \overline{u^{\prime}}}{\partial z}\right)^{2}\right]+\overline{u^{\prime} v^{\prime} f} \\
& \frac{1}{2} \frac{\partial \overline{v^{\prime 2}}}{\partial t}+\overline{v^{\prime} w^{\prime}} \frac{\partial \bar{v}}{\partial z}=-\frac{1}{\rho} \overline{v^{\prime} \frac{\partial p^{\prime}}{\partial y}}-\frac{\partial}{\partial z} \overline{w^{\prime} v^{\prime 2}} \\
& +\frac{1}{2} \nu\left(\frac{\partial^{2} \overline{v^{\prime 2}}}{\partial z^{2}}\right)-v\left[\left(\frac{\partial v^{\prime}}{\partial x}\right)^{2}+\left(\frac{\partial v^{\prime}}{\partial y}\right)^{2}+\left(\frac{\partial v^{\prime}}{\partial z}\right)^{2}\right]-\overline{u^{\prime} v^{\prime} f}
\end{aligned}
$$




$$
\begin{aligned}
& \frac{1}{2} \overline{\frac{\partial w^{\prime 2}}{\partial t}}=-\frac{1}{\rho} \overline{w^{\prime} \frac{\partial p^{\prime}}{\partial z}}-\frac{\partial}{\partial z} \overline{w^{\prime 3}}+\frac{1}{2} \nu\left(\frac{\partial^{2} \overline{w^{\prime 2}}}{\partial z^{2}}\right) \\
& -v\left[\left(\frac{\partial \mathrm{w}^{\prime}}{\partial \mathrm{x}}\right)^{2}+\left(\frac{\partial \overline{\mathrm{w}^{\prime}}}{\partial \mathrm{y}}\right)^{2}+\left(\frac{\partial \overline{\mathrm{w}^{\prime}}}{\partial \mathrm{z}}\right)^{2}\right]
\end{aligned}
$$

Note that the derivatives with respect to $\mathrm{x}$ and $\mathrm{y}$ of the turbulent velocities instantaneously can well be different from zero, so that a variance of these derivatives exists.

Of considerable interest is the role played by the Coriolis force. It acts as a redistributing agent, provided that $\overline{u^{\prime} v^{\prime}}$ exists, removing energy from the $v$ component and moving it into the $u$ component of the turbulent energy. However, for a constant-thickness layer with constant velocities in planes $\mathrm{z}=$ constant, the turbulence components are uncorrelated in such planes, and consequently $\overline{u^{\prime} v^{\prime}}$ must be zero.

Since the mean flow is steady, it follows that the turbulence must be steady also. Summing the energy of all components yields the total energy balance. If we rearrange the viscous term and introduce tensor notation, the energy equation becomes

$$
\overline{\mathrm{u}^{\prime} \mathrm{w}^{\prime}} \frac{\partial \overline{\mathrm{u}}}{\partial \mathrm{z}}+\overline{\mathrm{v}^{\prime} \mathrm{w}^{\prime}} \frac{\partial \bar{v}}{\partial z}=-\frac{1}{\rho} \frac{\partial}{\partial \mathrm{z}} \overline{\left[\mathrm{w}^{\prime}\left(\mathrm{p}^{\prime}+\rho \mathrm{q}^{2}\right]\right.}+\nu \frac{\partial}{\partial \mathrm{x}_{\mathrm{i}}} \overline{\mathrm{u}_{\mathrm{j}}^{\prime}\left(\frac{\partial \mathrm{u}_{\mathrm{i}}^{\prime}}{\partial \mathrm{x}_{\mathrm{j}}}+\frac{\partial \mathrm{u}_{\mathrm{j}}^{\prime}}{\partial \mathrm{x}_{\mathrm{i}}}\right)}-\epsilon
$$

in which

$$
\epsilon=\nu \overline{\left(\frac{\partial u_{i}^{\prime}}{\partial x_{j}}+\frac{\partial u_{j}^{\prime}}{\partial x_{i}}\right) \frac{\partial u_{j}^{\prime}}{\partial x_{i}}}
$$

is the dissipation and where the summation in the viscous terms has to be extended over both $i$ and $j$. Furthermore,

$$
q^{2}=\frac{1}{2}\left(u^{\prime 2}+v^{\prime 2}+w^{\prime 2}\right)
$$

is the kinetic energy of the turbulent motion, and the pressure term has been rewritten using the equation of continuity. It is usually assumed that the first two terms on the right, the energy-redistribution terms, are small quantities that can be neglected. The two terms on the left represent the generation of turbulent energy by interaction of the turbulent shearing stresses with the mean motion. To this approximation the equation can be expressed, in vector notation, by

$$
\tau \cdot \frac{\partial \overline{\mathrm{v}}}{\partial \mathrm{z}}=\rho \epsilon
$$




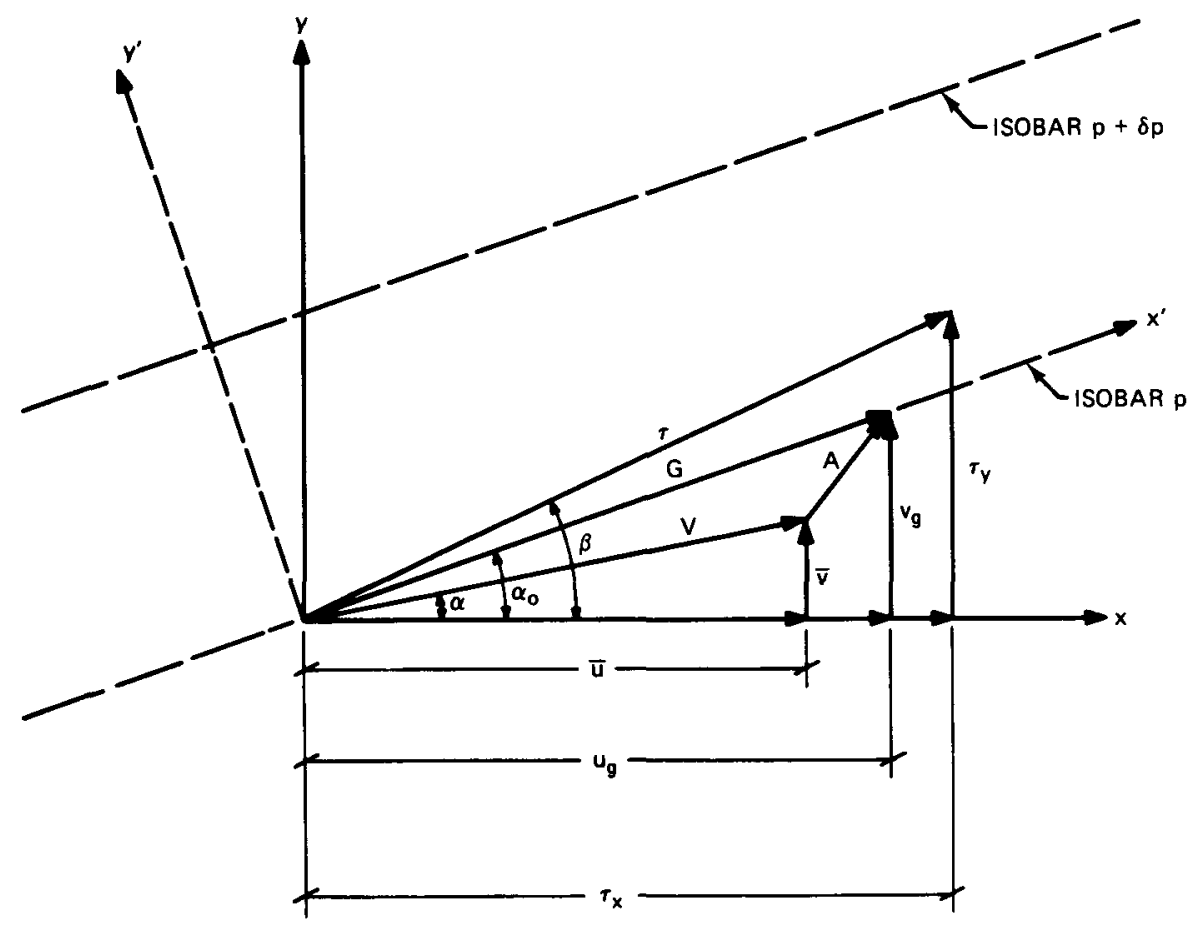

Fig. 1.2 Definitions of a planetary coordinate system in the $x-y$ plane. The $x-y$ coordinates are oriented with $x$ in the direction of the ground shear, the $x^{\prime}-y^{\prime}$ coordinates have the $x^{\prime}$-axis tangential to the isobar.

where $\tau$ is the shear-force vector per unit area. In absolute values it follows that, for a coordinate system oriented with the $\mathrm{x}$-axis in the direction of the ground shear stress as in Fig. 1.2 and with the notation of Fig. 1.2,

$$
\rho \epsilon=|\tau|\left|\frac{\partial \bar{v}}{\partial z}\right| \cos \left(\alpha^{\prime}-\beta\right)
$$

where $\alpha^{\prime}$ is the angle between the $\mathrm{x}$-axis and the vector $\partial \mathrm{v} / \partial \mathrm{z}$.

To gain more information from this equation, we need to find an independent relation for the angle $\alpha^{\prime}$. Such a relation can be found by postulating that the total dissipation of the boundary layer is a maximum. This assumption is occasionally made in attempts to close the set of turbulence equations, such as in the theoretical model of Malkus (1956). In general, its validity cannot be established (see Reynolds and Tiederman, 1967).

Since $\epsilon$ is always positive, a condition for maximum dissipation is that locally the dissipation has a maximum compatible with the dynamics of the problem. This evidently is obtained when the angle $\left(\alpha^{\prime}-\beta\right)$ between shear stress and velocity 
gradient $\dagger$ is zero because by definition $|\tau|$ and $|\partial v / \partial z|$ are independent of $\beta$ and $\alpha$. Interestingly, this result is a basic assumption which is made in applying mixing-length theories to the planetary boundary layer (see Blackadar, 1962). Recent numerical calculations of Deardorff (1969) based on an analytical model which yielded realistic results for integral parameters, like the drag coefficient of the surface, also appear to confirm this conclusion.

Further progress in obtaining a solution for Eqs. 1.19 and 1.20 can only be made if the factors of proportionality in the relation between shear stress and velocity gradient are known. Such a relation can take the form of a function of $\mathrm{z}$ describing the dependency of the eddy viscosity on height (Prandt1, 1965; Blackadar, 1962) or can postulate a height dependency of the angle between $\tau$ and the vector $\mathbf{A}=\mathbf{G}-\mathbf{v}$ in Fig. 1.2 (Lettau, 1962, 1970).

\section{THE PLANETARY BOUNDARY LAYER}

\section{The Basic Equations}

The equations of motion for the planetary boundary layer are conveniently discussed with reference to a coordinate system whose $\mathrm{x}$-axis points into the direction of the shear stress at the ground. We may then introduce a shear-stress vector as shown in Fig. 1.2,

$$
\tau=\tau_{\mathrm{x}} \mathrm{i}+\tau_{\mathrm{y}} \mathrm{j}
$$

whose $\mathrm{y}$ component equals zero and whose $\mathrm{x}$ component is equal to $\tau_{0}$ at $\mathrm{z}=0$. The components $\tau_{\mathrm{x}}$ and $\tau_{\mathrm{y}}$ are interpreted as kinematic quantities, stresses per unit mass, which are related to the velocity field by

$$
\tau_{\mathrm{x}}=\nu \frac{\partial \bar{u}}{\partial \mathrm{z}}-\overline{\mathrm{u}^{\prime} \mathrm{w}^{\prime}}
$$

and

$$
\tau_{\mathrm{y}}=\nu \frac{\partial \overline{\mathrm{v}}}{\partial \mathrm{z}}-\overline{\mathrm{v}^{\prime} \mathrm{w}^{\prime}}
$$

$\dagger$ In the meteorological literature it is customary to denote the velocity gradient by the term "wind shear." This confusing terminology shall be avoided as much as possible, but, when the term "shear" is used alone, it is implied to mean "gradient." 
In terms of these quantities, the governing Eqs. 1.19 and 1.20 become

$$
\begin{aligned}
& -f \bar{v}=-\frac{1}{\rho} \frac{\partial \bar{p}}{\partial x}+\frac{d \tau_{x}}{d z} \\
& +f \bar{u}=-\frac{1}{\rho} \frac{\partial \bar{p}}{\partial y}+\frac{d \tau_{y}}{d z}
\end{aligned}
$$

The pressure gradient is set up by the geostrophic field and is directed normal to the isobars of weather maps. It is useful to define the geostrophic wind components $u_{g}$ and $v_{\mathrm{g}}$ as those wind velocities which exist at an elevation where the shear-stress gradients can be neglected, so that, as indicated in Fig. 1.2,

$$
\begin{gathered}
u_{g}^{2}+v_{g}^{2}=G^{2} \\
G=u_{g} i+v_{g} \mathbf{j}=G \cos \alpha_{0} \mathbf{i}+G \sin \alpha_{0} \mathbf{j}
\end{gathered}
$$

where

$$
u_{g}=-\frac{\partial \bar{p}}{\partial y} \frac{1}{\rho f} \quad v_{g}=\frac{\partial \bar{p}}{\partial x} \frac{1}{\rho f}
$$

so that Eqs. 1.32 and 1.33 become

$$
\begin{aligned}
-f\left(\bar{v}-v_{g}\right) & =\frac{d \tau_{x}}{d z} \\
f\left(\bar{u}-u_{g}\right) & =\frac{d \tau_{y}}{d z}
\end{aligned}
$$

At the edge of the surface layer, the geostrophic wind is parallel to the isobars, but inside the surface layer the direction of the wind vector is modified by the shear stresses in such a way that in the northern hemisphere (where $f$ is positive) the velocity vector is rotated to the left of the geostrophic wind vector.

\section{Laminar Ekman Spirals}

A solution of the system Eqs. 1.36 and 1.37 requires that a relation between $\tau$ and $\mathbf{v}$ be found. For laminar flow, this relation is stated in Eqs. 1.30 and 1.31, where the turbulent stresses are equal to zero, and the well-known solution of Ekman (see Batchelor, 1967) is obtained:

$$
\begin{gathered}
\mathrm{u}_{\mathrm{E}}=\mathrm{G}\left(1-\mathrm{e}^{-\mathrm{kz}} \cos \mathrm{kz}\right) \\
\mathrm{v}_{\mathrm{E}}=\mathrm{Ge}^{-\mathrm{kz}} \sin \mathrm{kz}
\end{gathered}
$$


with

$$
\mathrm{k}=\sqrt{\frac{|\mathrm{f}|}{\nu}}
$$

Here the velocity components are given in the primed coordinate system of Fig. 1.2 whose $y$-axis lies in the direction of the negative geostrophic pressure gradient. The solution is found by putting $u_{E}-G=A e^{\lambda 2}$ and $v_{E}=B e^{\lambda z}$. If this solution is inserted into Eqs. 1.36 and 1.37, with the laminar part of Eqs. 1.30 and 1.31 used for shear stresses, it is seen that a solution satisfying the boundary conditions can only be found if $\lambda^{4}=-(f / \nu)^{2}$. Among the four complex roots of $\lambda$, the two that have a positive real part cannot satisfy the boundary conditions, and the two that have a negative real part lead to Eqs. 1.38 and 1.39. The solution is in the form of a spiral (as shown in Fig. 1.3a) whose velocity vector at the ground is rotated clockwise by $45^{\circ}$ from the direction of the pressure gradient.

The angle of $45^{\circ}$ exceeds any of the angles observed in wind spirals of the atmospheric surface layer. We find distributions whose hodographs are more like the one shown in Fig. $1.3 \mathrm{~b}$ because the air flow is turbulent. In turbulent flow it is not possible to relate the turbulent stresses of Eqs. 1.30 and 1.31 to mean flow quantities on the basis of first principles. Empirical assumptions will have to be made, on the basis of experience with laboratory flows, and some of these will be discussed in a later section. It is, however, possible to obtain some important results on the nature of the velocity distributions in the planetary boundary layer by considerations of the asymptotic behavior of the profiles alone without previous knowledge of the shear-stress distribution.

\section{Dimensional Considerations and the Inner Law of the Planetary Boundary Layer}

The energy required to maintain the motion near the ground is ultimately taken from the air flow outside the surface layer, and consequently the conditions above the planetary boundary layer will set a velocity scale for the wind profile in the surface layer. Near the ground, however, it is more likely that the flow is determined by the interaction of velocity field and shear stresses, much as in zero-pressure-gradient turbulent boundary layers in an aerodynamic environment, where the momentum lost at the ground is balanced by a gain of momentum through entrainment of higher energy fluid near the edges of the boundary layer.

Under these conditions, in close analogy to the turbulent boundary layer along a flat plate, we need to consider the atmospheric surface layer as made up of two sublayers: (1) an outer sublayer, whose mechanics are determined by the interaction of pressure gradient and Coriolis force and whose characteristics are determined mostly by the conditions near the edge of the surface layer, and (2) an inner sublayer, whose structure is determined by the flux of momentum to the ground which depends 

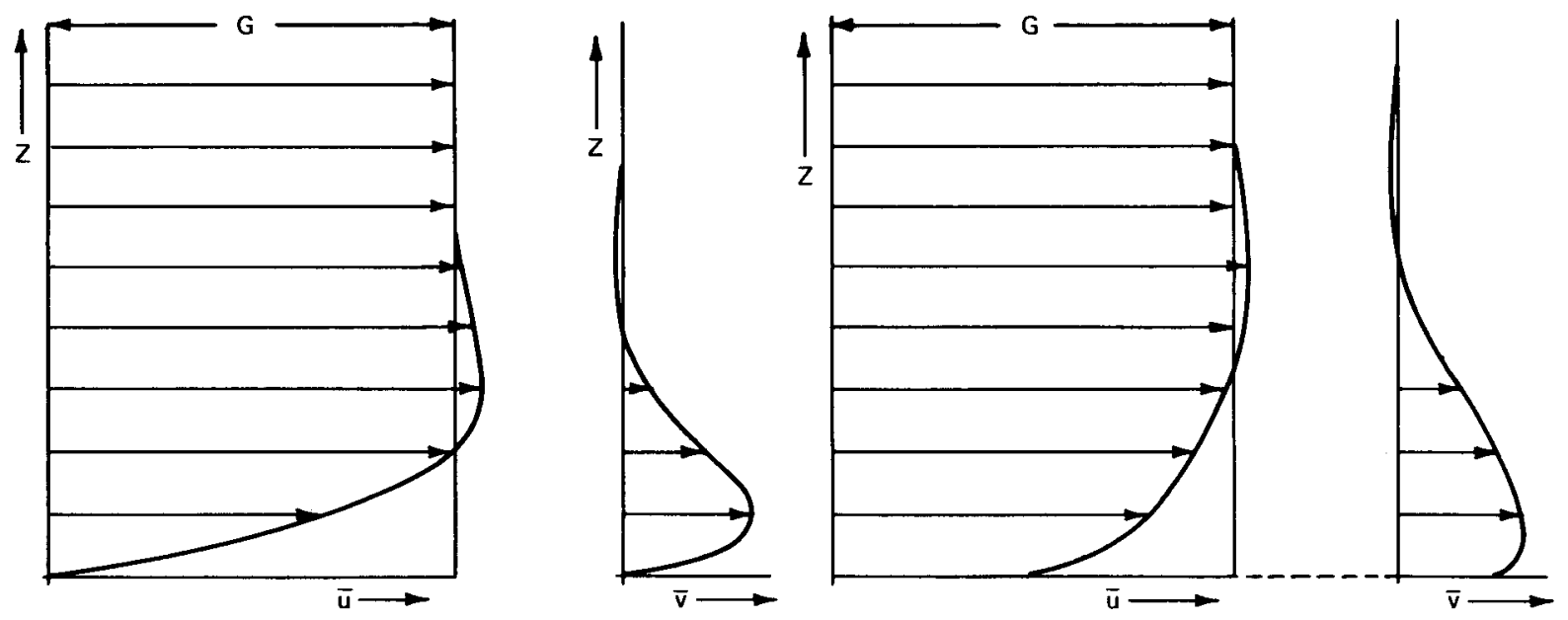


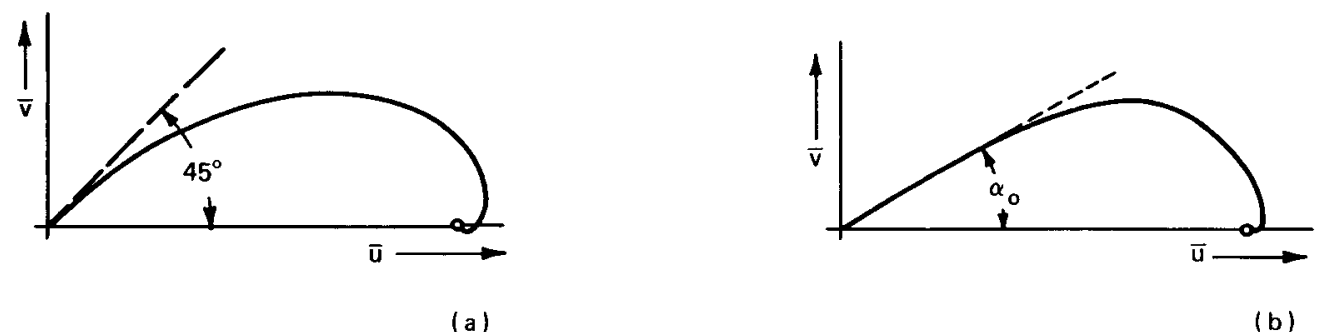

(b)

Fig. 1.3 (a) The Ekman spiral in laminar flow, exact solution. (b) Typical example of the Ekman spiral in turbulent flow (from Prandtl, 1965). 
on the nature of the ground surface. This conclusion permits us to construct a velocity profile for the atmospheric boundary layer, much as was done by Clauser (1954) for the turbulent boundary layer along a flat plate. In doing this, we will use the $x-y$ coordinate system of Fig. 1.2.

Over uniform terrain the velocity distribution at zero pressure gradient is fully specified for the inner layer by a velocity scale, $u_{*}$, and a length scale, $z_{0}$, such that (with overbar dropped from mean velocities)

$$
\frac{\mathrm{u}}{\mathrm{u}_{*}}=\mathrm{f}_{\mathrm{x}}\left(\frac{\mathrm{z}}{\mathrm{z}_{0}}\right) \frac{\mathrm{v}}{\mathrm{u}_{*}}=\mathrm{f}_{\mathrm{y}}\left(\frac{\mathrm{z}}{\mathrm{z}_{0}}\right)
$$

where $f\left(z / z_{0}\right)$ is a universal function and $u_{*}$ is defined by Eq. 1.15. The length $z_{0}$ is a characteristic of the surface, is independent of the flow conditions, and must be given as part of the boundary conditions.

A simple estimate of the asymptotic form of the functions $f_{y}\left(z / z_{0}\right)$ can be obtained by remembering that in the chosen coordinate system the shear stress component $\tau_{\mathrm{y}}$ is zero at the wall, and so we find, by developing $\mathrm{v}$ in a Taylor series about $\mathrm{z}=0$, that asymptotically the velocity distribution is given by a straight line

$$
\frac{\mathrm{v}}{\mathrm{u}_{*}}=0+\frac{\tau_{\mathrm{y}}(0)}{\mu} \mathrm{z}+\ldots
$$

or

$$
\frac{\mathrm{v}}{\mathrm{u}_{*}}=0
$$

Thus $f_{y}=0$ near $z=0$. From Eq. 1.36 it follows also that to the same approximation the shear stress, $\tau_{\mathrm{x}}$, varies linearly with $\mathrm{z}$ and is equal to $\rho \mathrm{u}_{*}^{2}$ near the ground.

At the outer edge of the surface layer, the flow is determined by a velocity scale and a length scale that pertain to the outer layer only. Csanady (1967) and Blackadar and Tennekes (1968) have shown that the velocity scale for the outer layer is also the shear velocity, $u_{*}$, and that a length scale is readily found to be equal to $h \sim u_{*} / f$.

A nondimensional velocity-distribution law of the form $u / u_{*}$ and $v / v_{*}$ would depend for $z=h$ on the geostrophic velocity $G$. Since $G / u_{*}$ presumably depends on the Reynolds number and configuration of the surface roughness, $u / u_{*}$ could not be a universal function. However, the velocity-defect laws

$$
\frac{\mathrm{u}-\mathrm{u}_{\mathrm{g}}}{\mathrm{u}_{*}}=\mathrm{g}_{\mathrm{x}}\left(\frac{\mathrm{zf}}{\mathrm{u}_{*}}\right) \quad \frac{\mathrm{v}-\mathrm{v}_{\mathrm{g}}}{\mathrm{u}_{*}}=\mathrm{g}_{\mathrm{y}}\left(\frac{\mathrm{zf}}{\mathrm{u}_{*}}\right)
$$

do not have these shortcomings because both functions approach zero at $z=h$. Equations 1.41 and 1.43 depend on three parameters: the Reynolds number, which determines the ratios $u_{g} / u_{*}, v_{g} / u_{*}$, etc., a Rossby number, defined to 


$$
\mathrm{Ro}_{0}=\frac{\mathrm{z}_{0} \mathrm{f}}{\mathrm{u}_{*}}
$$

and the height parameter $\xi=\mathrm{z} / \mathrm{z}_{0}$ For large Reynolds numbers, which almost always occur under natural conditions, the Reynolds number effect is no longer noticeable, and the profiles depend on Ro and $\xi$ only

The asymptotic Eqs 142 and 1.43 require that, in the "matched region" where both equations are to hold, the velocity distribution be loganithmic. This fact, which was already determined by Clauser (1954) for the similar case of the boundary layer along a flat plate, has been formalized for the case of the Ekman layer by Blackadar and Tennekes (1968) who used the method of matched expansions (van Dyke, 1964) We will derive the velocity distribution in the matched region by means of this method

In the matched layer, Eqs 1.41 and 143 are valid, so that

$$
\frac{u_{g}}{u_{*}}+f_{x}\left(\frac{z}{z_{0}}\right)=g_{x}\left(\frac{z}{h}\right)
$$

Since both $\mathrm{h}=\mathrm{u}_{*} / \mathrm{f}$ and $\mathrm{u}_{\mathrm{g}} / \mathrm{u}_{*}(=\mathrm{a}$ drag coefficient $)$ depend on the Rossby number only, we can write

$$
\frac{u_{g}}{u_{*}}+f_{x}(\xi)=g_{x}(\phi \xi)
$$

where the notations $\phi(\operatorname{Ro})=z_{0} / h$ and $\xi=z / z_{0}$ have been used The parameters $\phi$ and $\xi$ are the independent variables of the problem By differentiating Eq. 1.46 first with respect to Ro and then with respect to $\xi$ and eliminating $\mathrm{g}_{\mathrm{x}}^{\prime}(\phi \xi)$ from the two resulting independent equations, we obtain

$$
\xi f_{x}^{\prime}(\xi)=\frac{d}{d R o} \frac{u_{g}}{u_{*}}\left(\frac{d \ln \phi}{d \operatorname{Ro}}\right)^{-1}
$$

The left side is a function of $\xi$, the right side is a function of Ro only, consequently both sides are equal to a constant, $1 / \mathrm{k}$, say, and integration yields

$$
\begin{gathered}
\frac{\mathrm{u}}{\mathrm{u}_{*}}=\frac{1}{\mathrm{k}} \ln \frac{\mathrm{z}}{\mathrm{z}_{\mathrm{o}}} \\
\frac{\mathrm{u}_{\mathrm{g}}}{\mathrm{u}_{*}}=-\frac{1}{\mathrm{k}}\left(\ln \frac{\mathrm{z}_{0}}{\mathrm{~h}}+\mathrm{A}\right)
\end{gathered}
$$

Simılarly, equatıng Eqs. 1.42 and 1.43 for v yields

$$
-\frac{v_{g}}{u_{*}}=g_{y}\left(\frac{z}{h}\right)=+B / k
$$


where the constant $+B / k$ is universal and must be found from experimental data. The constant was chosen in this form to correspond to the terminology employed by Csanady (1967). Essentially identical results, but with $\mathrm{G} / \mathrm{fz}_{0}$ used instead of $\mathrm{u}_{*} / \mathrm{fz}_{0}$, were also given by Kasanski and Monin (1960) and by Csanady (1967). The coefficients $A$ and $B$ are constants that must be determined by experiments, and this is conveniently done using experimental data for the deviation angle, $\alpha_{0}$, of the geostrophic wind from the surface shear direction,

$$
\sin \alpha_{0}=\frac{+v_{g}}{G}=-\frac{B u_{*}}{k G}=-\frac{B}{k} c_{g}
$$

and for the geophysical drag coefficient,

$$
c_{g}^{2}=\frac{u_{*}^{2}}{G^{2}}=c_{g}^{2}(R o)
$$

The evaluation of these parameters from measured profiles is not an easy matter because small errors in velocity profiles may be reflected in large errors in the parameters and because profiles must be taken over great heights and will therefore show potentially large errors due to terrain inhomogeneities of the approach area. Accurate profile evaluations were made, in particular by Lettau and his associates (Lettau, 1950, 1957, 1959, 1962) (Lettau and Hoeber, 1964), who evaluated a number of selected wind profiles for this purpose, and from these we can infer that for $\mathrm{k}=0.4, \mathrm{~B}$ is approximately 4.3 and $\mathrm{A}$ is about 1.7 . Csanady (1967) has given the following relations, in terms of the Rossby number $G / z_{0} f$ :

$$
\sin \alpha_{0}=-10.7 \mathrm{c}_{\mathrm{g}}
$$

and

$$
\ln \frac{G}{f z_{0}}=0.4\left(\frac{1}{c_{g}^{2}}-115\right)^{1 / 2}+1.15 \log \left(\frac{1}{c_{g}^{2}}\right)-1.52
$$

to express the relation between geostrophic drag coefficient and Rossby number and angle between ground shear and isobars. The experimental data of Lettau and his coworkers have been plotted according to Eqs. 1.51 and 1.52 in Figs. 1.4 and 1.5 where, in addition to the data used by Csanady, the Lakewood data of Johnson (1956) are indicated. The Lakewood data, taken over an extensive and dense forest, yield surprisingly large friction factors, which are, however, in agreement with the general trend of the data analyzed by Blackadar (1962) and by Russian workers (Zilitinkevich et al., 1967). Average curves through the collection of Blackadar's data are also shown in Figs. 1.4 and 1.5. 


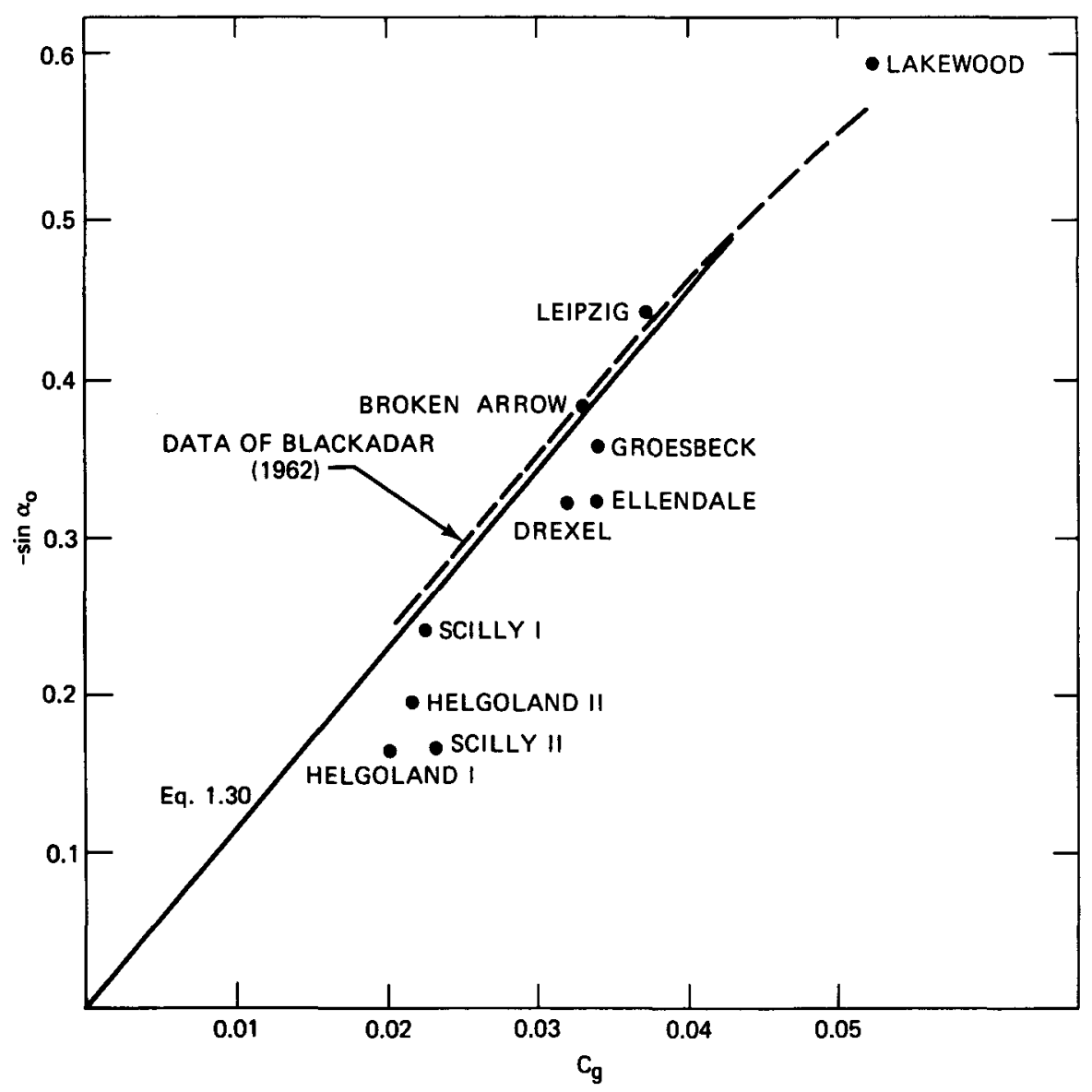

Fig. 1.4 Angle of ground stress against isobars, as function of geostrophic drag coefficient (from Csanady, 1967). The dotted line has been constructed from the average curves given by Blackadar (1962).

\section{The Outer Part of the Planetary Boundary Layer}

It is of great importance that asymptotically the velocity-distribution law near the wall become logarithmic law, Eq. 1.48, and, since at the ground $\mathrm{dv} / \mathrm{dz}=0$ as well as $v=0$, it is likely that over the lowest portion of the atmospheric boundary layer the influence of the Coriolis force can be neglected. The angle $\alpha_{0}$ as observed in moderate latitudes does not exceed about $23^{\circ}$; therefore it is not likely that its influence is felt in the region near the ground. In the outer flow, however, the $\mathrm{v}$ component has this important dynamic function: It is a major component in the motion down the pressure gradient which is the cause of reducing and ultimately eliminating the pressure gradients, thus equalizing atmospheric highs and lows. Therefore we need to 


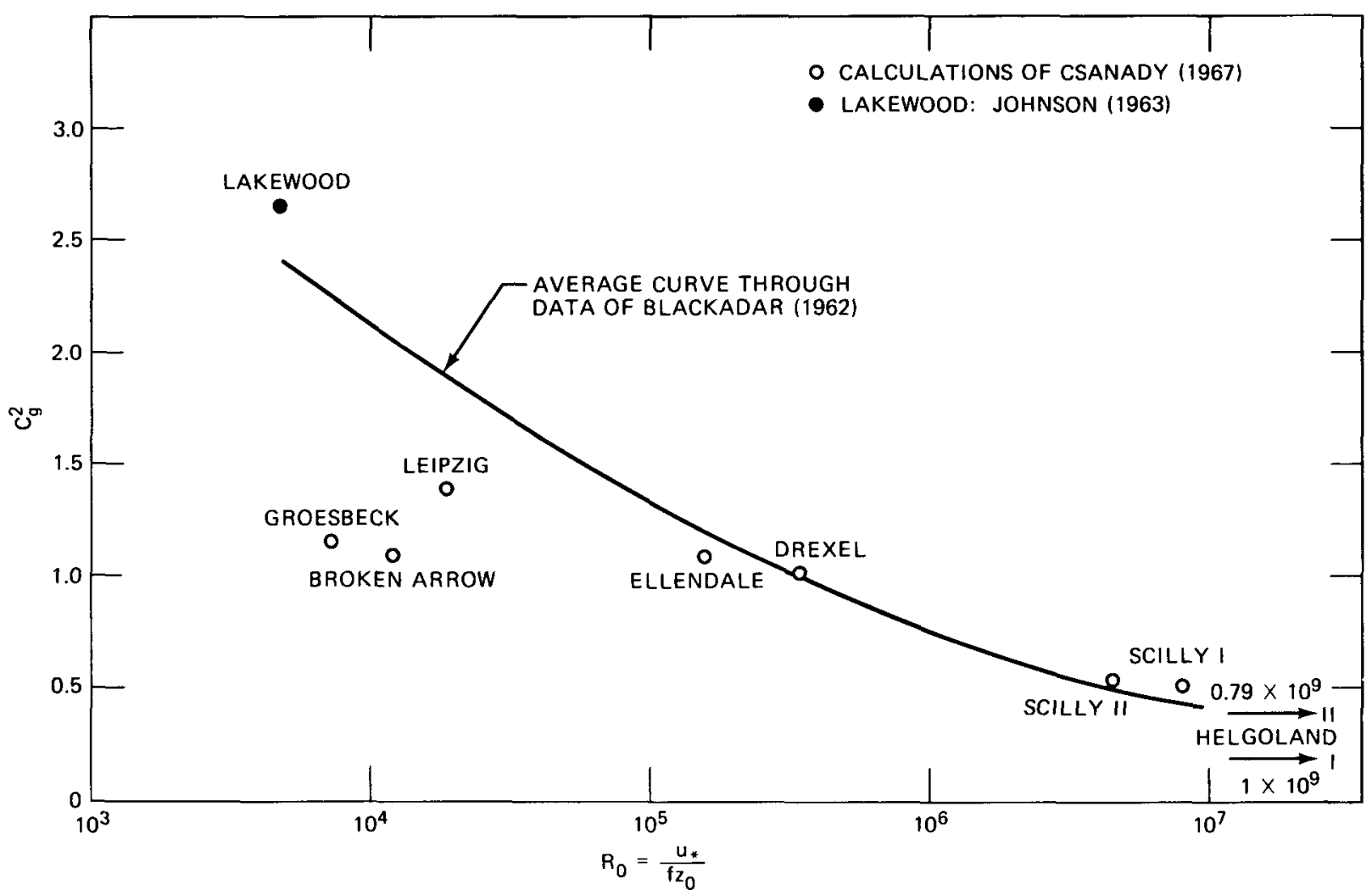

Fig. 1.5 Geostrophic drag coefficient as function of surface Rossby number. The points are from Csanady (1967), and the curve is from the average curves of Blackadar (1962). 
consider both velocity components in the outer flow and to find a solution for Eqs. 1.36 and 1.37 valid for the outer layer.

At the edge of the planetary boundary layer, the distributions of velocity and shear stress smoothly join the flow aloft, where $\tau=0, u=u_{g}$, and $v=v_{g}$ everywhere independent of height. This condition requires that at the edge of the planetary boundary layer the first derivatives of the shear stresses, according to Eqs. 1.36 and 1.37 , be zero. With this result the behavior of the flow field can be inferred by expanding $\tau$ in a Taylor series about $\mathrm{z}=\mathrm{h}$ and calculating the resulting profiles. The calculations are aided if it is assumed that the velocity vector and the shear-stress vector are parallel at all elevations $z$. These conditions have been used by Swinbank (1969) for determining the flow field near $h$.

When

$$
\tau=\tau \cos \alpha \mathbf{i}+\tau \sin \alpha \mathbf{j} \quad \tau^{2}=\tau_{\mathrm{x}}^{2}+\tau_{\mathrm{y}}^{2}
$$

and

$$
\mathrm{v}=\mathrm{V} \cos \alpha \mathrm{i}+\mathrm{V} \sin \alpha \mathbf{j} \quad \mathrm{V}^{2}=\mathrm{u}^{2}+\mathrm{v}^{2}
$$

as well as

$$
\mathbf{G}=\mathrm{G} \cos \alpha_{0} \mathbf{i}+\mathrm{G} \sin \alpha_{0} \mathbf{j}
$$

where $\alpha_{0}$ is the angle between the ground wind direction and the direction of the geostrophic wind (positive counterclockwise), then the equations of motion, Eqs. 1.36 and 1.37 , become

$$
\begin{aligned}
& -\mathrm{f}\left(\mathrm{V} \sin \alpha-\mathrm{G} \sin \alpha_{0}\right)=\frac{\partial}{\partial \mathrm{z}}(\tau \cos \alpha) \\
& -\mathrm{f}\left(\mathrm{V} \cos \alpha-\mathrm{G} \cos \alpha_{0}\right)=\frac{\partial}{\partial \mathrm{z}}(\tau \sin \alpha)
\end{aligned}
$$

Multiplying Eq. 1.58 by $\cos \alpha$ and Eq. 1.59 by $\sin \alpha$, and then adding, eliminates $V$, and with the identities

$$
\begin{aligned}
& \cos \alpha \frac{\partial}{\partial z} \tau \cos \alpha=\cos ^{2} \alpha \frac{\partial \tau}{\partial z}-\tau \cos \alpha \sin \alpha \frac{\partial \alpha}{\partial z} \\
& \sin \alpha \frac{\partial}{\partial z} \tau \sin \alpha=\sin ^{2} \alpha \frac{\partial \tau}{\partial z}+\tau \cos \alpha \sin \alpha \frac{\partial \alpha}{\partial z}
\end{aligned}
$$

it follows that 


$$
\frac{\partial \tau}{\partial z}=-f G \sin \left(\alpha-\alpha_{0}\right)
$$

To complete the solution, Swinbank (1969) expanded $\tau$ in a Taylor series about $z=h$, and since at $\mathrm{z}=\mathrm{h}$ we have $\alpha=\alpha_{0}$, it follows that:

$$
\tau=\left.\frac{1}{2} \frac{\partial^{2} \tau}{\partial z^{2}}\right|_{h}(\mathrm{z}-\mathrm{h})^{2}=-\left.\mathrm{fG} \frac{\mathrm{d} \alpha}{2 \mathrm{dz}}\right|_{\mathrm{h}}(\mathrm{h}-\mathrm{z})^{2}
$$

where Eq. 1.61 has been used to eliminate $\partial^{2} \tau /\left.\partial \mathrm{z}^{2}\right|_{\mathrm{h}}$. Thus the shear-stress distribution is quadratic in $(h-z)$, whereas the angle $\left(\alpha-\alpha_{0}\right)$ depends on $z$ as

$$
\sin \left(\alpha-\alpha_{0}\right)=+\left.\frac{\mathrm{d} \alpha}{\mathrm{dz}}\right|_{\mathrm{h}}(\mathrm{z}-\mathrm{h})
$$

The linear distribution of $\sin \left(\alpha-\alpha_{0}\right)$ and the quadratic dependency of $\tau$ on $(h-z)$ were surprisingly well confirmed over most of the planetary boundary profile, at least for the well-known "Leipzig" profile used by Lettau (1950) and Swinbank (1969).

We should note that the questionable assumption $\mathrm{v}$ parallel to $\tau$ was used only in the derivation of Eq. 1.61 and that Eq. 1.61 was used only for yielding the derivatives of $\tau$ at $\mathrm{z}=\mathrm{h}$. Therefore it is useful to see if there are not other ways of deriving Eq. 1.61 without Swinbank's assumption. Inspection of Eqs. 1.36 and 1.37 reveals that the vector $\mathrm{d} \tau / \mathrm{dz}$ is perpendicular to the vector $\mathrm{A}=\mathrm{G}-\mathbf{v}$ in Fig. 1.2, and thus Eq. 1.61 is seen to imply that the vector $\mathbf{A}$ is perpendicular to $\mathbf{v}$. Since this condition has to hold near $\mathrm{z}=\mathrm{h}$ only, it follows that the conclusions arrived at by Swinbank would result if near $\mathrm{z}=\mathrm{h}$ the wind veered in such a way that the hodograph of $\mathbf{v}$ is a semicircle with radius $1 / 2 \mathrm{G}$ centered at $1 / 2 \mathrm{G}$ on the $\mathrm{x}^{\prime}$-axis of Fig. 1.2. It is significant that this condition is not far from observed data (for example, see Lettau, 1970) and also corresponds to the laminar flow solution. Apparently Eq. 1.61 is valid near $z=h$, even though Swinbank's assumption leading to it may be incorrect.

The quantities $d \alpha /\left.d z\right|_{h}$ and $h$ must be determined to complete the solution. Expressions for them are readily found by assuming that the outer profile is everywhere determined by its asymptotic form and thus also in the overlap region of the inner and outer profiles. Then Eq. 1.53 can be considered applicable, and it follows for $\mathrm{z}=0$ from Eq. 1.63:

$$
\sin \alpha_{0}=\left.\frac{\mathrm{d} \alpha}{\mathrm{dz}}\right|_{\mathrm{h}} \mathrm{h}=-10.7 \frac{\mathrm{u}_{*}}{\mathrm{G}}
$$

and the nondimensional distributions of $\sin \left(\alpha-\alpha_{0}\right)$ and $\tau$ become, respectively,

$$
\sin \left(\alpha-\alpha_{0}\right)=+10.7 \frac{u_{*}}{G}\left(\frac{h-z}{h}\right)
$$


and

$$
\frac{\tau}{\mathrm{u}_{*}^{2}}=+\frac{\mathrm{10} 7}{2} \frac{\mathrm{hf}}{\mathrm{u}_{*}}\left(\frac{\mathrm{h}-\mathrm{z}}{\mathrm{h}}\right)^{2}
$$

But $h=\alpha u_{*} / f, \dagger$ and thus, with a factor of proportionality of $\alpha$,

$$
\frac{\tau}{\mathrm{u}_{*}^{2}}=+54 \alpha\left(\frac{\mathrm{h}-\mathrm{z}}{\mathrm{h}}\right)^{2}
$$

Since at $\mathrm{z}=0, \tau / \mathrm{u}_{*}^{2}=1$, the factor $\alpha$ is equal to $1 / 54$, and thus the following expressions for $\mathrm{h}$ and $\mathrm{d} \alpha /\left.\mathrm{dz}\right|_{\mathrm{h}}$ are obtained

$$
\mathrm{h}=\frac{1}{54} \frac{\mathrm{u}_{*}}{\mathrm{f}}
$$

and

$$
\left.\frac{\mathrm{d} \alpha}{\mathrm{dz}}\right|_{\mathrm{h}}=-589 \frac{\mathrm{f}}{\mathrm{G}}
$$

It is remarkable that, when this estimate for $h$ is applied to the Leipzig wind profile, for which Csanady (1967) gives $f=11410^{-4} \mathrm{sec}^{-1}$ and $u_{*}=063 \mathrm{~m} / \mathrm{sec}$, it follows that $\mathrm{h}=1030 \mathrm{~m}$, in almost perfect agreement with the value of $\mathrm{h}=1070 \mathrm{~m}$ inferred by Swinbank (1969) for the same data The value also is in good agreement with $\mathrm{h}=02 \mathrm{u}_{*} / \mathrm{f}$, assumed by Clarke (1969) as an average value for a large number of profiles observed near Kerang, Victoria, and Hay, New South Wales, Australia, over sites that have been described by Swinbank and Dyer (1968) Individual profiles were found, however, to deviate considerably, especially for thermally stratıfied flow

\section{VELOCITY DISTRIBUTION NEAR THE GROUND}

The derivation of the logarithmic law by asymptotic matching cannot give any information on the thickness of the layer in which it is valid In a turbulent boundary layer along a smooth flat plate, $1 \mathrm{e}$, for measurements like those of Klebanoff (1955), the logarithmic part of the profile extends to a distance of about $015 \mathrm{~h}$. In pipe flow, on the other hand, the logarithmic law is valid almost to the center of the pipe, and

$\dagger$ Note that instead of setting $\mathrm{h} \propto \mathrm{u}_{*} / \mathrm{f}$, we could have left $\mathrm{h}$ unspecified, except by postulating that $\mathrm{z}_{0} / \mathrm{h}=\mathrm{F}$ (Ro) only Then Eqs 167 and 168 would have yielded not only the numerical constant $\alpha$ but also the functional form of $h$ as well 
the distance to which it is applicable is therefore about $h$. One reason for this difference in pipe and flat-plate boundary-layer flows lies in the difference of the turbulence levels. In turbulent pipe flow, the turbulence is everywhere, whereas near the edge of the boundary layer the flow is intermittently turbulent, i.e., the turbulent eddies are sharply separated from the nonturbulent fluid that is entrained from outside the boundary layer. A velocity-measuring probe, such as a hot wire held at a fixed distance from the wall, will therefore sometimes see a turbulent signal and sometimes a calm flow (Townsend, 1956; Sandborn, 1959). The calm flow is more likely at a velocity closer to the free-stream velocity because, owing to the lack of turbulence, there is no strong shear coupling between adjacent strata and the entrained fluid does not feel the presence of the boundary. The turbulent fluid, on the other hand, is strongly coupled to lower strata. Suppose for a moment that in the turbulent portion the logarithmic law is valid and that in the calm fluid the velocity equals the free-stream velocity. The mean flow then would show a profile that has velocities lying between the free-stream velocity and the logarithmic velocity. Such velocities are indeed observed. If the free stream were also turbulent, then it is likely that the mean velocity profile would be logarithmic over a larger part of the boundary layer than for the case of very low free-stream turbulence. That this is what happens was shown by Wieghardt (1944).

The atmosphere outside the lower atmospheric boundary layer is usually not free of turbulence. This stems partly from the fact that topographic features and nonhomogeneities of the upwind terrain generate turbulence that remains for some time in the boundary layer. Therefore we expect a larger portion than the lowest $15 \%$ of the atmospheric boundary layer to be logarithmic. Unambiguous evidence for this behavior is, however, not available.

The logarithmic velocity-distribution law is determined by the parameters $\mathrm{k}, \mathrm{z}_{\mathbf{0}}$, and $\mathrm{u}_{*}$. As long as $\mathrm{k}$ is a universal constant, its value is known; $\mathrm{z}_{0}$ is a property of the roughness, and $u_{*}$ follows from the upper boundary parameters, as indicated by Eq. 1.54. However, when the lower boundary layer is treated independently of the planetary boundary as a whole, as is done in most micrometeorological work, the shear velocity, $\mathfrak{u}_{*}$, cannot be predicted from the boundary conditions. Therefore a frequent suggestion has been the use of a drag coefficient referred to the velocity at some fixed height, say $10 \mathrm{~m}$, i.e., to define a drag coefficient $c_{10}=u_{*}^{2} / u_{10}$, where $u_{10}$ is the reference velocity at $10 \mathrm{~m}$. But in a layer that is described by the logarithmic velocity distribution, this is just another way of expressing the roughness height $z_{0}$, as can readily be seen by inserting $z=10 \mathrm{~m}$ and $\mathrm{u}=\mathrm{u}_{10}$ into Eq. 1.48. Consequently, if $z_{0}$ is given, it follows that the ratio $u_{10} / u_{*}$ is also given at each value of $z$. Thus, if a particular $u_{10}$ is assumed for a given $z_{0}$, the wind profile is fully specified. A better way of obtaining $u_{*}$ is direct measurement of the wall shear stress, but this requires elaborate experimental equipment such as drag plates. In micrometeorological work it is usual to infer the shear at the ground from measured profiles of wind-velocity distribution, a procedure that works satisfactorily in neutrally stratified boundary layers. 


\section{Velocity Profile Along Smooth Walls}

Experimental evidence has shown that the logarithmic velocity distribution, Eq. 1.48, yields a valid description of mean velocity conditions in at least the lowest 16 to $20 \mathrm{~m}$ of the atmospheric boundary layer, down to very small distances from the ground. How far down depends on the configuration of the surface. When the surface is very smooth with no geometric obstructions hindering the motion of the air, viscosity might affect the flow very near the ground because the no-slip condition at the ground does not permit the existence of turbulence fluctuations. Under these conditions the shear stress at the ground is purely viscous, given by

$$
\rho u_{*}^{2}=\tau_{0}=\left.\rho \nu \frac{\partial \bar{u}}{\partial z}\right|_{0}
$$

and the velocity distribution can be developed in a Taylor series expansion,

$$
\overline{\mathrm{u}}(\mathrm{z})=\overline{\mathrm{u}}(0)+\left.\frac{\partial \overline{\mathrm{u}}}{\partial \mathrm{z}}\right|_{0} \mathrm{z}+\left.\frac{1 \partial^{2} \overline{\mathrm{u}}}{2 ! \partial \mathrm{z}^{2}}\right|_{0} \mathrm{z}^{2}
$$

For very small $\mathrm{z}$ the higher order terms can be neglected, and, in view of the no-slip condition and Eq. 1.70, the velocity profile becomes

$$
\frac{\overline{\mathrm{u}}}{\mathrm{u}_{*}}=\frac{\mathrm{u}_{* \mathrm{Z}} \mathrm{z}}{\nu}+0\left[\left(\frac{\mathrm{u}_{*} \mathrm{z}}{\nu}\right)^{2}\right]
$$

where $0\left[\left(\mathrm{u}_{*} \mathrm{z} / \nu\right)^{2}\right]$ stands for the higher order terms. We notice that a length scale, $\delta_{\mathrm{e}} \sim \nu / \mathrm{u}_{*}$ (the thickness of the laminar sublayer), arises in a natural fashion for the velocity distribution near the wall, whereas the velocity scale is $u_{*}$. In the outer part of the inner layer, the velocity distribution is logarithmic but is governed by the same length and velocity scales, so that

$$
\frac{\overline{\mathrm{u}}}{\mathrm{u}_{*}}=\frac{1}{\mathrm{k}} \ln \frac{\mathrm{zu}_{*}}{v}+\mathrm{C}
$$

where the factor of proportionality between $z_{0}$ and $v / u_{*}$ has been absorbed into $C$. Experimental evidence, obtained in many laboratory tests, has resulted in a value of $\mathrm{C}$ of about 5.6 (Clauser, 1954), but it depends to some extent on the choice of $\mathrm{k}$.

The most commonly accepted value of $\mathrm{k}$ is 0.4 , and we will use it whenever a numerical value is needed. Experimental evidence exists for values of $\mathrm{k}$ ranging from as low as 0.2 to as high as 0.8 . However, no systematic dependency of $\mathrm{k}$ on Reynolds number, roughness, or admixtures to the flow has been found. A recent study of the value of $\mathrm{k}$, by Slotta (1963), failed to lend support to any value of $\mathrm{k}$ that differs much from 0.4 in zero-pressure-gradient flow. 
The logarithmic profile, Eq 148 , naturally cannot be valıd for $z$ smaller than some positive number, because below a certain level the velocities would be negative Experience has shown that the transition region between the velocity profiles in Eqs 172 and 173 is rather thin, and so it is possible to specify the distance from the wall at which the valıdity of Eq 173 begins by the intersection of Eqs 172 and 173 A typical experimental curve is shown in Fig 16 , which is taken from Hinze (1959) The corresponding distance $z=\delta_{\mathrm{e}} \sim 11 \nu / \mathrm{u}_{*}$ is called the viscous sublayer thickness, where the viscous sublayer is that region in which the effect of molecular viscosity dominates the exchange processes

This situation is in effect not changed if the ground surface is not fixed but moving, for example, if the velocity profile over a surface of water is considered The wind induces a current, with a component $u_{s}$ in the direction of the wind profile, relative to which Eq 173 is valid Relative to a stationary observer, the local velocity $\mathrm{u}_{\mathrm{e}}=\mathrm{u}+\mathrm{u}_{\mathrm{s}}$, where $\mathrm{u}$ is given by Eq 173 When the logarithmic law is applied to experimental data of $u_{e}$, we find that

$$
\frac{\mathrm{u}_{\mathrm{e}}}{\mathrm{u}_{*}}=\frac{1}{\mathrm{k}} \ln \frac{\mathrm{zu}_{*}}{\nu}+\mathrm{C}+\frac{\mathrm{u}_{\mathrm{s}}}{\mathrm{u}_{*}}=\frac{1}{\mathrm{k}} \ln \frac{\mathrm{zu}_{*}}{\nu}+\mathrm{C}_{\mathrm{e}}
$$

so that the effective coefficient $C_{e}$ is increased by $u_{s} / u_{*}$ The surface then appears smoother than a smooth solid surface, and this may explain the fact that over the ocean a $z_{0}$ value smaller than $\sim 11 \nu / u_{*}$ is often observed (Roll, 1965) That for a smooth water surface the constant $C_{e}$ is indeed equal to $56+u_{s} / u_{*}$ was shown by Plate, Chang, and Hidy (1969) for wind blowing over the water surface in a laboratory channel

\section{Velocity Profiles Over Rough Surfaces}

Very few surfaces of natural ground can qualify to be called smooth Aurport runways or highways, perhaps reasonably uniform snow covers, and the surface of bodies of water at low winds exhibit smooth surface character The particular distinction of smooth surface character is that the length scale $z_{0}$ depends on the wind velocity Most ground surfaces are aerodynamically rough, a state that is determined by arrays of individual rough elements protruding from the surface, from which the air flow separates For separation to occur, it is of course necessary that the local velocity and the roughness element combine to form a Reynolds number that exceeds a critical value If the height of the roughness is denoted by $d$, then it is reasonable to assume that the reference velocity for the critical Reynolds number is given by the velocity at the top of the element, or the average velocity over its height, and the height $d$ Experience has shown that the critical Reynolds number is very small, so that separation is avoided only if $d$ is very small Then the velocity distribution over the height of the element is described by Eq 172 , and consequently the critical Reynolds number is given to 




Fig. 1.6 Theoretical velocity distribution in the transition region of a turbulent boundary layer, compared with experimental data by Reichardt for channel flow and by Laufer for pipe

flow [H Reichardt, Zeitschrift fuer Angewandte Mathematik und Mechanik, 31: 208 (1951)

J. Laufer, National Advisory Committee for Aeronautics Technical Report No. 1174, 1954] ; from Hinze (1959). 


$$
\operatorname{Re}_{\text {crit }}=\frac{u_{*} \mathrm{~d}}{\nu} \mid \text { critical }
$$

This number will depend on the geometry and arrangements of the roughness elements. When separation occurs, the shear velocity is determined by the form drag of the individual roughness elements. Since the form drag depends on the shape of the element as well as on the mutual interference of the wakes of adjacent elements, it is not surprising that there does not exist a one-to-one correspondence of roughnesselement height and length scale $z_{0}$. Only when shape and arrangement of roughness elements are kept constant while the scale of the elements is changed can there be a relation between $z_{0}$ and the dimension of the roughness element. Typical for this behavior is the classical uniform sand roughness of Nikuradse (see Schlichting, 1968) for which $z_{0}=d / 30$, where $d$ is the diameter of the sand. The sand behavior is aerodynamically rough only when

$$
\frac{\mathrm{u}_{*} \mathrm{~d}}{\nu} \geqslant 70
$$

which implies that, in that Reynolds number range, separation occurs at all elements.

The Reynolds number, Eq. 1.76, can be interpreted as the ratio of the roughness height $d$ to the thickness of the viscous sublayer (Schlichting, 1968). This has led to the interpretation that a surface is aerodynamically rough when the roughness element "penetrates the viscous sublayer," and it is smooth if the sublayer covers the roughness element. This concept is, however, not particularly satisfactory, because it ignores the essential interactions of the roughness element with the flow, while relating rough flow behavior only to the height of the elements rather than to their aerodynamic characteristics.

An interesting way of interpreting Eq. 1.48 for a rough surface is in terms of Eq. 1.74. We may write Eq. 1.48 in the equivalent form

$$
\frac{\mathrm{u}}{\mathrm{u}_{*}}=\frac{1}{\mathrm{k}} \ln \frac{\mathrm{zu}_{*}}{v}+\mathrm{C}+\frac{\mathrm{u}_{\mathrm{s}}}{\mathrm{u}_{*}}
$$

where

$$
-\frac{\mathrm{u}_{\mathrm{s}}}{\mathrm{u}_{*}}=\frac{1}{\mathrm{k}} \ln \frac{\mathrm{z}_{0} \mathrm{u}_{*}}{\nu}+\mathrm{C}
$$

in which $u_{s}$ is a sort of slip velocity (Hama, 1954). The quantity $u_{s} / u_{*}$ is positive if the surface moves in the direction of the flow; it is negative if the surface moves in the opposite direction. A rough surface is thus seen to correspond in its effect to a smooth surface that moves against the wind at the velocity $\mathrm{u}_{\mathrm{s}}$ given by Eq. 1.78 , or the air flow is slipping at the surface at a velocity $u_{s}$ instead of being zero at the surface. 
For many vegetative covers that are not too closely spaced, we also find a single length that describes the surface roughness. In that case Eq. 1.48 is found valid in the form

$$
\frac{\mathrm{u}}{\mathrm{u}_{*}}=\frac{1}{\mathrm{k}} \ln \frac{\mathrm{z}-\mathrm{d}_{0}}{\mathrm{z}_{0}}
$$

where $\mathrm{d}_{0}$ is the zero-plane displacement, introduced by Rossby and Montgomery (1935). The extensive experiments of Paeschke (1937) on natural crops have yielded the $z_{0}$ values given in Table 1.1. They correspond approximately to a relation between

Table 1.1

PROFILE PARAMETERS OF CROPS*

\begin{tabular}{llrcc}
\hline \multicolumn{1}{c}{ Crop } & $\mathrm{z}_{\mathbf{0}}, \mathrm{cm}$ & $\mathrm{h}_{\mathrm{c}}, \mathrm{cm}$ & $\mathrm{c}_{(5 \mathrm{~m}) \times 10^{3}}$ & $\mathrm{c}_{(10 \mathrm{~m}) \times 10^{3}}$ \\
\hline Plane, snow covered & 0.49 & 3 & 3.25 & 3.9 \\
Grassy surface & 1.73 & 10 & 4.90 & 6.2 \\
Flat country & 2.14 & 10 & 5.5 & 6.9 \\
Low grass & 3.20 & 20 & 6.4 & 8.3 \\
High grass & 3.94 & 30 & 7.2 & 9.3 \\
$\quad$ Wheat & 4.5 & 130 & 8.1 & 10.7 \\
$\quad$ Tan and Ling: & 3 to 4.8 & & & \\
$\quad$ (in Lemon, 1963) & & & & \\
$\quad$ Beets & 6.4 & 45 & 8.8 & 11.9 \\
\hline
\end{tabular}

*After Paeschke (1937).

crop height $h_{c}$ and $z_{0}$ given to $z_{0}=0.15 h_{c}$. This relation was also found to hold for croplike elements that were tested in a wind tunnel by Plate and Quraishi (1965). A set of field data similar to Paeschke's has been published by Priestley (1959). It is based on profiles that had been measured by Deacon (1953) and agrees approximately with the data of Paeschke. However, $z_{0}$ values quoted by Priestley for snow and similar surfaces are considerably lower than the ones given by Paeschke, perhaps because they correspond to approximately aerodynamically smooth or transitional surfaces. It is possible that the same surface type has a value of $z_{0}$ for the low-velocity conditions of Priestley, which is in the smooth regime, whereas at higher velocities the flow is rough. An increase in wind speed increases the Reynolds number and may lead to a rough surface with a larger effective value of $z_{0}$.

Some of Deacon's $z_{0}$ values are shown in Fig. 1.7 plotted against $h_{\mathfrak{c}}$, together with wind-tunnel values on real and artificial grass by Chamberlain (1966), wind-tunnel data on artificial trees by Hsi and Nath (1968) and data obtained from wind profiles over tall vegetations by Kung (1961). Kung described his data by an empirical relation:

$$
\log z_{0}=-1.24+1.19 \log h_{c}
$$




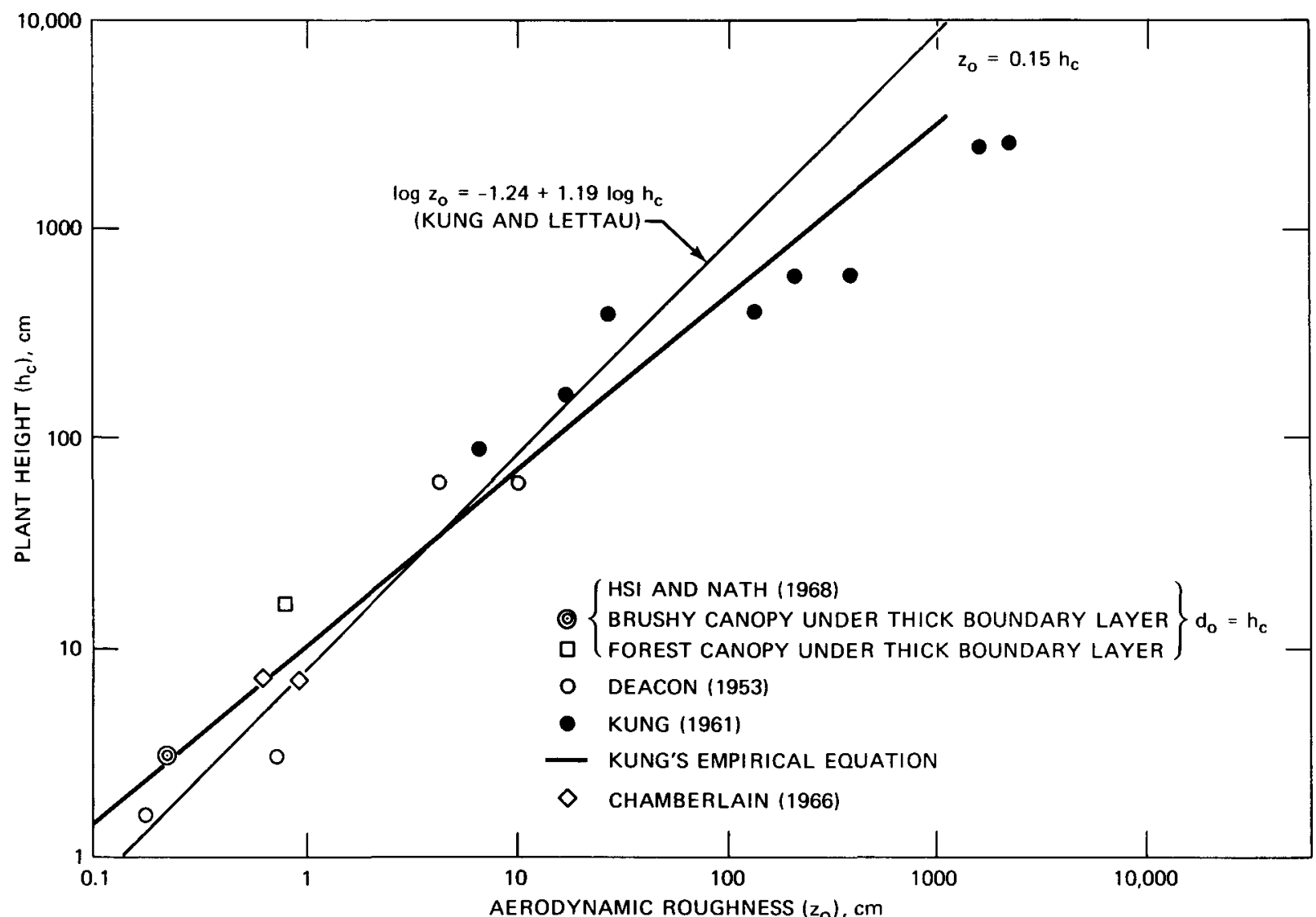

Fig. 1.7 Relation between aerodynamic roughness $z_{0}$ and roughness height $h_{c}$ : field (Deacon, Kung) and laboratory (Hsi and Nath, Chamberlain) observations. 
which is indicated in Fig 17 The equation of Kung 1s, however, not particularly satisfactory because it is not dimensionally homogeneous Also, it is likely that the derivation of the $z_{0}$ values for large plant heights is serrously affected by the measurement technique of Kung Another empirical equation, by Tanner and Pelton (1960) $\log z_{0}=\log h_{c}-088$, is readily seen to reduce to $z_{0}=014 h_{c}$, in good agreement with the relation given above, and the line drawn into Fig 17

A change in $\mathrm{z}_{0}$ with wind speed may also occur for a fully rough surface A special case is a wavy water surface where the wind produces waves that in turn affect the wind profile Other surfaces, for example, certain crops, form flexible coverings and are deformed by strong winds It is a common observation that a surface covered with long grass becomes smooth in appearance at high winds because the individual grass leaves are bent away from the wind High leaves are more exposed to the wind drag and are therefore bent more strongly than short leaves, with the result that the surface becomes more level as the wind increases This is found reflected in the $z_{0}$ values (Deacon, 1953) which decrease with increasing wind

The only crop listed by Paeschke which does not obey even approximately the relation $z_{0}=015 h_{c}$ is wheat This is no experimental error, since Paeschke's $z_{0}$ value of $45 \mathrm{~cm}$ is in very close agreement with the range of $z_{0}$ values from 3 to $48 \mathrm{~cm}$ obtained for wheat by Tan and Ling (1n Lemon, 1963) It should be attributed to the denseness of the crop, which makes the surface of the crop smoother than a cover of more widely spaced stalks A method has been designed by Lettau (1969) for incorporating roughness-element spacing and shape into an equation for $\mathrm{z}_{0}$

The zero-plane displacement $\mathrm{d}_{0}$ in Eq 179 was introduced to account for the origin shift that must be expected to occur for rough surfaces It is readily seen that the ground elevation does not have any significance in the dynamics of the air flow above the roughness Typical is the flow over a forest There is no reason to assume that low trees with exactly identical crowns as high trees should have, with respect to the crowns, different origins for the profile of the above-mentioned wind velocity The zero-plane displacement has been found, for dense crops to an excellent approximation, equal to the cover height (or $d_{0}=h_{c}$ ) both in the field (Paeschke, 1937) and for a model crop in the wind tunnel (Plate and Qura1shı, 1965) In general, however, we must expect $d_{0}$ to differ from $h_{c}$ when the density of the roughness elements is sparse Flows over such arrangements are usually not fully rough, and special investigations, possibly in a laboratory environment, must be made for them The difficulties associated with defining a suitable zero-plane displacement in such cases are well known (for example, Sayre and Albertson, 1963, and the discussion of their paper)

The existence of a zero-plane displacement does not imply that the mean velocity profile is logarithmic for all $z>d_{0}$ The cited experıments by Paeschke (1937) and Plate and Quraishı (1965) indicate that the logarithmic law becomes valid roughly for $\mathrm{z}>2 \mathrm{~h}_{\mathrm{c}}$ as seen in the examples in Fig 18 Below this height the arr flow is determined by the nature of the roughness elements The flow within the roughness cover, the "canopy flow," is of some importance in its own right because it determines the microclimate within the plant cover, $1 \mathrm{e}$, the exchange processes of heat, gases, and moisture 


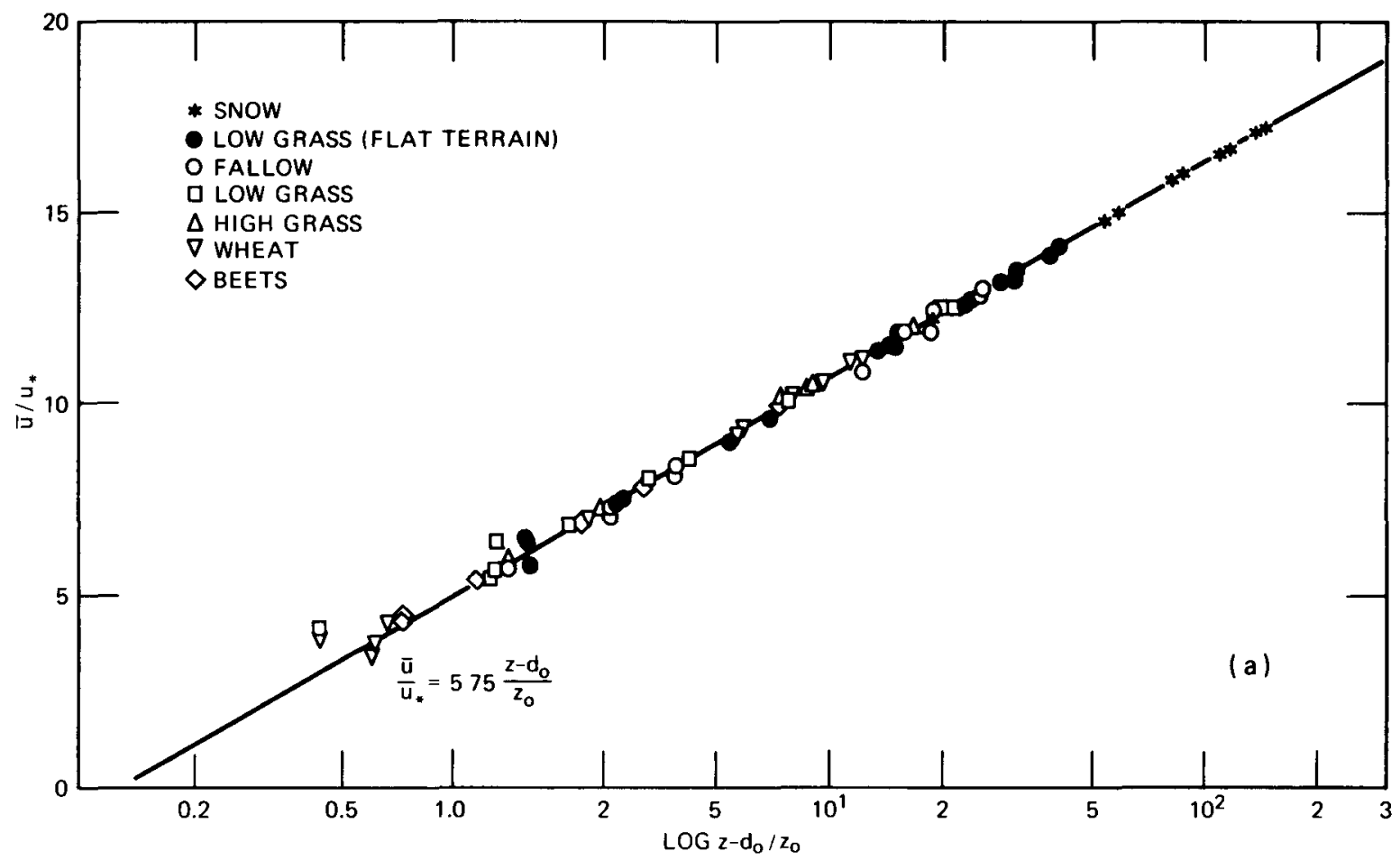

Fig. 1.8 Velocity profiles over crops: (a) field data of Paeschke (1937), where $d_{0}$ is the zero-plane displacement that is only approximately equal to the crop height $h_{c}$; (b) laboratory data of Plate and Quraishi (1965). 


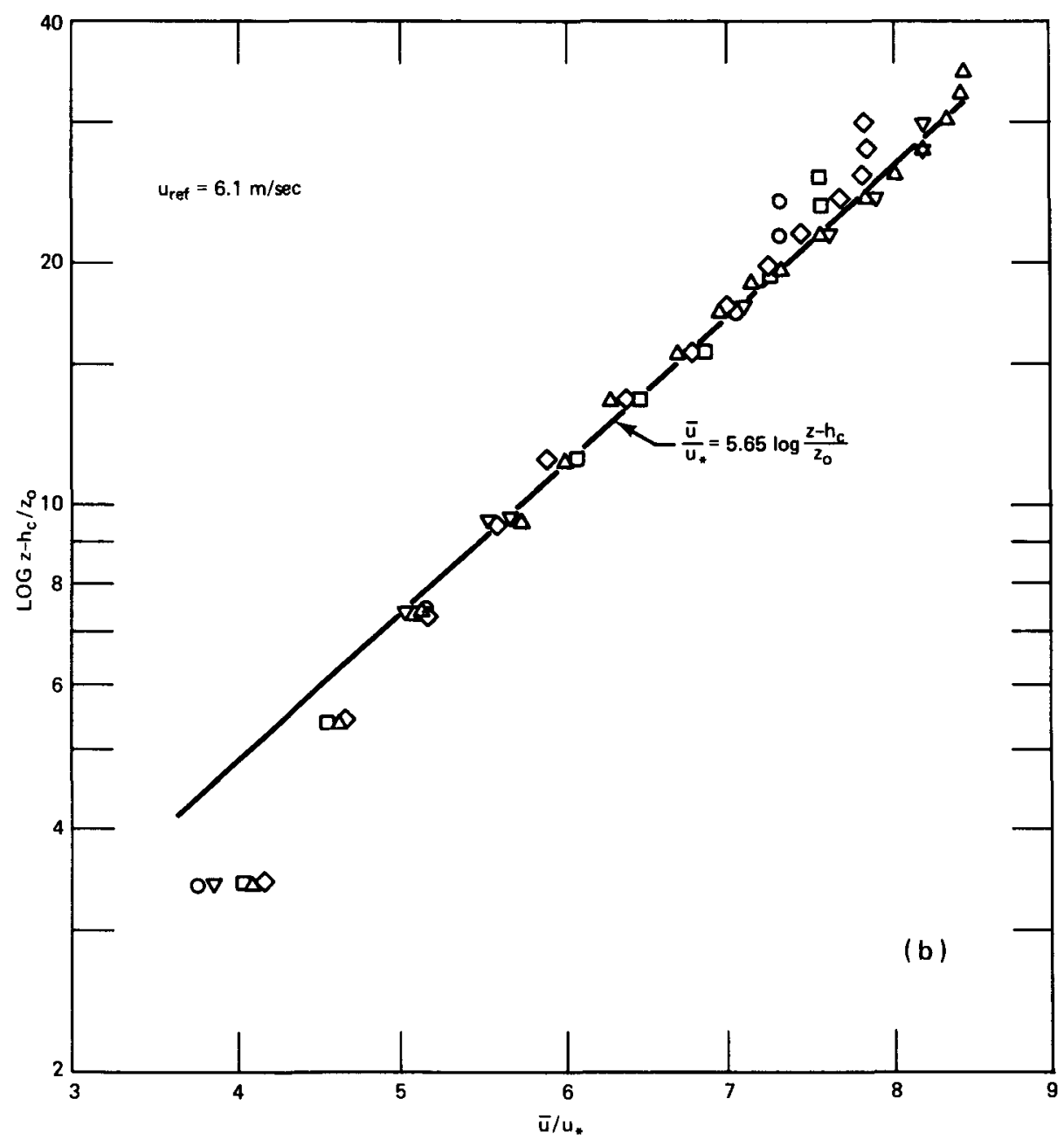

Fig. 1.8 (Continued)

\section{Canopy Flow}

To fully describe the wind field at a site covered with roughness elements, we need to also consider the flow between roughness elements, i.e., for $0<z<h_{c}$. From vegetative covers, or canopies, the flow between the roughness elements has received the name canopy flow. Canopy flow determines the microclimate in forest and crops, by governing exchange processes such as evaporation and diffusion of heat, insecticides, or pollutants. Quantitative expressions for the velocity profiles in the 
canopy must be based on a model for the interaction between canopy stems and leaves on the one side and wind shear on the other. For fairly densely spaced crops, Plate and Quraishi (1965) attempted to avoid the complications arising from the threedimensional nature of the flow by introducing similarity theory, i.e., by plotting $\vec{u} / u_{\text {ref }}$ vs. $z / z_{\text {ref }}$. The obvious reference length is the crop height $h_{c}$; for a reference velocity they chose the velocity $u_{h}$ at $z=h_{c}$. Figure 1.9 is a plot of field data from many different sources. We see that profiles pertaining to one particular type of crop, for example, the wheat profiles, are well-defined by a universal curve. It could be sufficient to specify the canopy flow by the type of crop that produces it. This method does not work, of course, when the eddy diffusivity or a turbulence quantity must be predicted for a roughness whose characteristics are not known. The least that needs to be known then is the shear-stress distribution in the canopy.

The shear-stress distribution inside vegetative covers depends on many factors, such as stalk spacing, leaf area exposed to the wind in the canopy, and shape and surface configuration of leaves. It is therefore very difficult to generate a general model applicable to all types of crops. Nevertheless, a few conclusions on the shear-stress distributions can be drawn. Consider the idealized crop of height $h_{c}$ shown in Fig. 1.10. When the flow is fully established and the pressure along the $\mathrm{x}$-axis is constant, then the shearing stress at the surface, $\rho \mathrm{u}_{*}^{2}$, is transmitted to the ground by the drag of the stalks and by friction at the ground.

In canopies the flow can be highly channeled, such as in man-planted crops. In this case the flow in between the rows obeys the simple shear-stress relation:

$$
\frac{\mathrm{d} \tau_{\mathrm{Xz}}}{\mathrm{dz}}=-\frac{2 \tau_{\mathrm{S}}}{\mathrm{b}}
$$

where $\tau_{\mathrm{xz}}$ is the average shear across the $\mathrm{x}-\mathrm{y}$ plane, and $\tau_{\mathrm{s}}$ is the shear stress in the $\mathrm{x}-\mathrm{z}$ plane, along the stalks. This equation serves to show that, among otherwise identical crops, more momentum is taken out of the flow by narrow rows than by wide rows. In general, the flow is not channeled; for random plant orientation the equation becomes

$$
\frac{\mathrm{d} \tau_{\mathrm{Xz}}}{\mathrm{dz}}=+\mathrm{D}
$$

where D is the drag force per unit volume of plant cover (Uchijima and Wright, 1964; Cionco, 1965, among many others). Finally, it is possible to express the drag by the aerodynamic formula

$$
\mathrm{D}=\rho \mathrm{c}_{\mathrm{d}} \mathrm{A}(\mathrm{z}) \cdot \mathrm{u}^{2}
$$

where $c_{d}$ is the drag coefficient and $A(z)$ is that area of the leaves which blocks the motion of the air flow. Practical models for solving Eqs. 1.82 and 1.83 depend on suitable assumptions for $\mathrm{A}(\mathrm{z}), \mathrm{c}_{\mathrm{d}}$, and the relation between shear stress and mean 


\begin{tabular}{|c|c|c|c|c|c|}
\hline \multicolumn{2}{|c|}{$\begin{array}{c}\text { STOLLER \& LEMON } \\
(1963)\end{array}$} & \multicolumn{2}{|c|}{$\begin{array}{c}\text { TAN \& LING } \\
(1961) \\
\end{array}$} & \multicolumn{2}{|c|}{$\begin{array}{l}\text { PAESCHKE } \\
(1937)\end{array}$} \\
\hline \multicolumn{2}{|c|}{ WHEAT } & \multicolumn{2}{|c|}{ CORN } & \multicolumn{2}{|c|}{ WHEAT } \\
\hline Symbol & $\mathrm{u}_{\mathrm{h}}, \mathrm{cm} / \mathrm{sec}$ & Symbol & $u_{n}, \mathrm{~cm} / \mathrm{sec}$ & Symbol & $u_{h}, \mathrm{~cm} / \mathrm{sec}$ \\
\hline \multirow{5}{*}{$\begin{array}{l}0 \\
0 \\
0 \\
0\end{array}$} & 373 & 0 & 200 & 0 & 95 \\
\hline & $\begin{array}{l}124 \\
217\end{array}$ & 0 & $\begin{array}{l}230 \\
292\end{array}$ & \multicolumn{2}{|c|}{$\begin{array}{c}\text { TAN \& LING } \\
(1961)\end{array}$} \\
\hline & 287 & $\oplus$ & 325 & \multicolumn{2}{|c|}{ WHEAT } \\
\hline & & $\theta$ & & Symbol & $u_{h}, \mathrm{~cm} / \mathrm{sec}$ \\
\hline & & & & $\Delta$ & $90-300$ \\
\hline
\end{tabular}

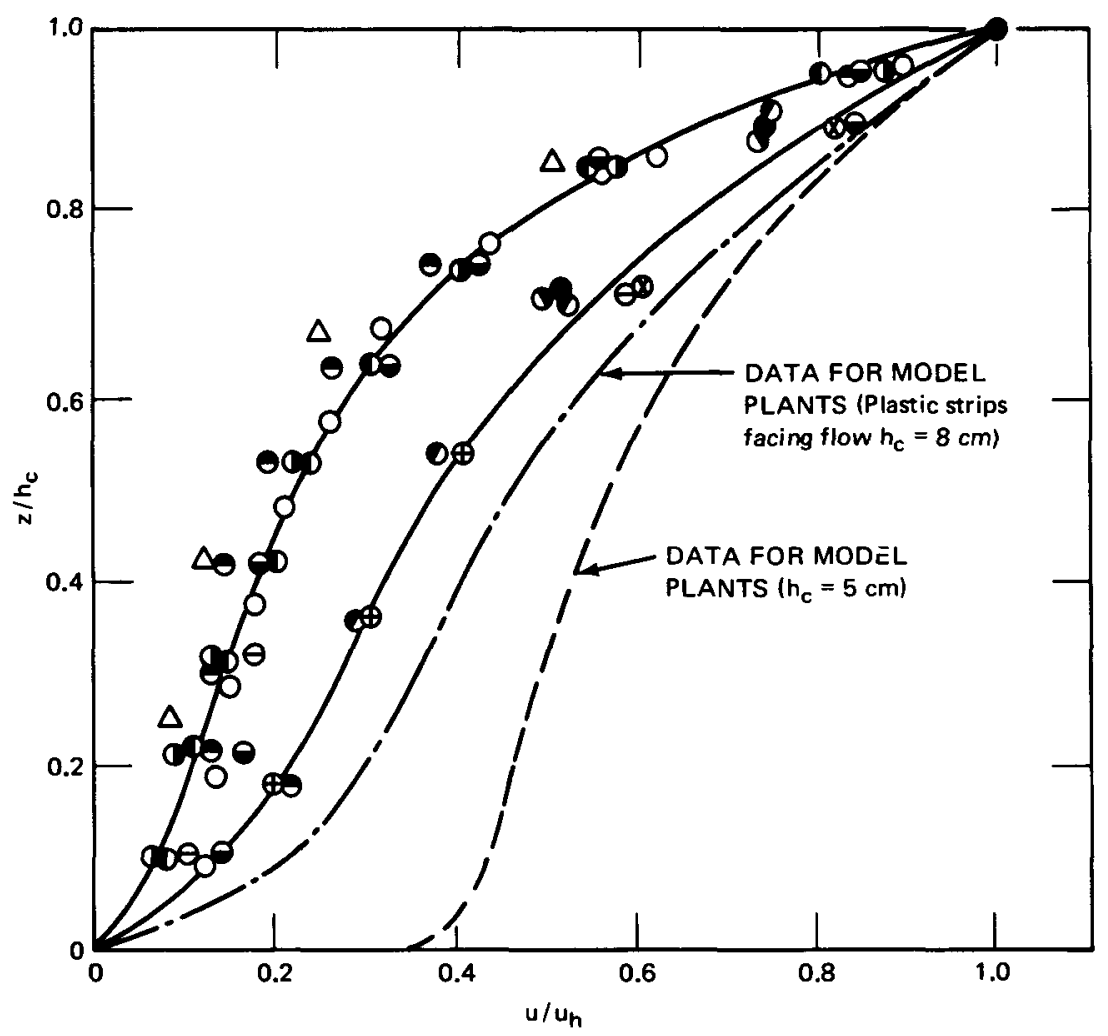

Fig. 1.9 Centerline velocity profiles between rows of crops. Laboratory data of Plate and Quraishi (1965) and field data from Paeschke, 1937; Tan and Ling, 1961; and Stoller and Lemon, 1963. 


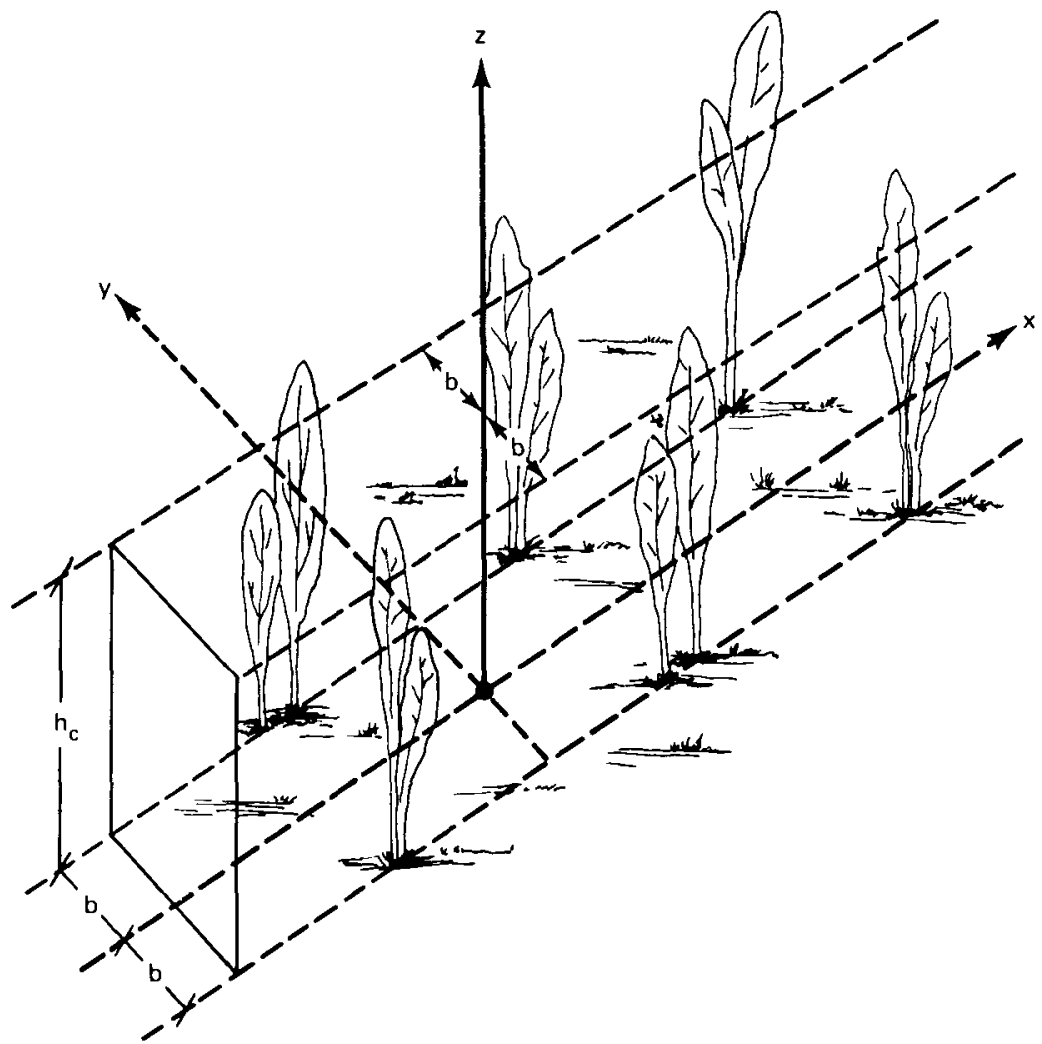

Fig. 1.10 Definition of the geometry of canopies.

velocity For $\mathrm{A}(\mathrm{z})$ it is possible to use the cumulative leaf area, an example of which is shown in Fig 111 for a mature cornfield (from Allen, Yokum, and Lemon, 1964) The drag coefficient is most frequently assumed constant, but Uchijıma and Wright (1964) found, from the analysis of many different wind profiles in a cornfield, that $\mathrm{c}_{\mathrm{d}}$ can vary over a very wide range, in their case from 0055 to 0542 This variation can be partly attributed to uncertainties in determining the aerodynamically active leaf area It is readily apparent that the leaf-area index is not a suitable measure of the aerodynamic resistance, since leaves will orient themselves in the direction of the wind or will cause additional drag by fluttering Under these conditions a rough approximation with a constant leaf area might be equally suitable, and when, in addition, the observed fact is used that the shear-stress distribution (or the velocity distribution) is approximately exponential, then Eqs 182 and 183 give also an exponential wind profile (or shear-stress distribution) Apparently this approximation leads to results of eddy viscosity $\epsilon$ which are in as good an agreement with observations (Uchiııma and Wright, 1964) as more elaborate models using a refined eddy-viscosity assumption and a variable leaf area (Cionco, 1965) 


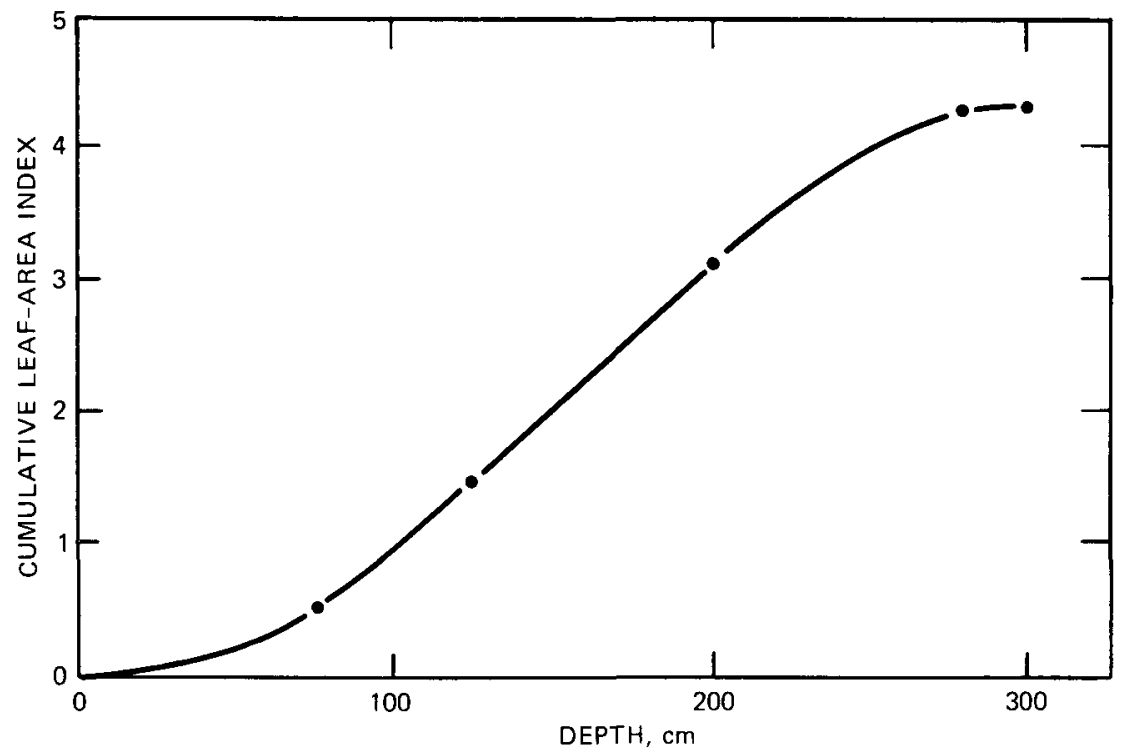

Fig. 1.11 Leaf-area index for corn as function of distance from the ground. The crop height is about $3 \mathrm{~m}$. From Allen, Yokum, and Lemon (1964).

\section{The Shear-Stress Distribution in the Planetary}

\section{Boundary Layer Near the Ground}

Equation 1.67 has been given as an estimate of the dependency of the shear stress on $z$ near the edge of the planetary boundary layer. Near the ground a relation for the shear stress can also be found by using the asymptotic forms of the equations of motion. As was shown, asymptotically, $v$ for $z \rightarrow 0$ becomes zero, and thus we find from Eq. 1.36 that

$$
\frac{\partial \tau_{\mathrm{X}}}{\partial \mathrm{z}}=+\mathrm{f} \mathrm{v}_{\mathrm{g}}
$$

which can be integrated to yield

$$
\frac{\tau_{\mathrm{x}}(\mathrm{z})}{\mathrm{u}_{*}^{2}}=1-\frac{\mathrm{fB}}{\mathrm{ku}} \cdot \mathrm{z}
$$

For $\mathrm{f}=1.14 \cdot 10^{-4} \mathrm{sec}^{-1}, \mathrm{u}_{*}=0.2 \mathrm{~m} / \mathrm{sec}$, and with $\mathrm{B}=4.3$ and $\mathrm{k}=0.4$, the shear stress is seen to decrease by about $10 \%$ in a distance of $10 \mathrm{~m}$. It is customary to neglect the variation of shear stress with height in the lowest layer of the atmosphere where the constant-stress assumption is assumed to imply that the shearing stress varies by less than $10 \%$ from its mean value at the ground. Lumley and Panofsky (1964) infer that 
this region has a thickness varying from 10 to $100 \mathrm{~m}$, depending on the ground shear stress, whereas Eq 185 would tend to lead, for the range of values of $u_{*}$ and $c_{g}$ that are usually encountered in the atmosphere, to a thickness of from 10 to $50 \mathrm{~m}$ This estimate is, however, low because the effect of $\bar{v}$ in decreasing the shear correction is not considered in Eq 185 , and it is possible that the thicknesses obtained by including $\bar{v}$ are in the range given by Lumley and Panofsky

Making the assumption of a layer of constant shear stress near the ground leads to two important consequences First, in all considerations of similarity, the distribution function of the shearing stress need not be considered since it is fully described by $\tau_{0}$ Second, a knowledge of such a simplified shear-stress distribution can aid in establishing the relation between the shear stress and the velocity field

The relation cannot be developed from first principles and must be established in an empirical manner For a fully viscous flow, the shear stress is related to the velocity gradient, $1 \mathrm{e}$,

$$
\tau=\mu \frac{\partial \mathrm{u}}{\partial \mathrm{z}}
$$

and in analogy to this equation, we can write for turbulent flows

$$
\tau=\mathrm{K}_{\mathrm{M}} \frac{\partial \overline{\mathrm{u}}}{\partial \mathrm{z}}
$$

where the quantity $\mathrm{K}_{\mathrm{M}}$ is termed Boussinesq's eddy viscosity, which has the dimension of kinematic viscosity, $1 \mathrm{e}, \mathrm{cm}^{2} / \mathrm{sec} A$ solution for $\overline{\mathrm{u}}$ requires an assumption about $\mathrm{K}_{\mathrm{M}}$ Clearly, an assumption $\mathrm{K}_{\mathrm{M}}=$ constant, analogous to the molecular viscosity, leads to a linear velocity distribution in a constant-stress layer, which is contrary to observations Rather, it must be expected that $\mathrm{K}_{\mathrm{M}}$ cannot be predicted, in general Not only does it depend on the location inside the flow field but also on the outer boundary conditions, $1 \mathrm{e}$, on the pressure gradient and the growth rate of the boundary layer In general, $\mathrm{K}_{\mathrm{M}}$ is a function of the development history of the boundary layer, and only in some special cases can it be expected that $\mathrm{K}_{\mathrm{M}}$ is predicted by local conditions only

For that part of the constant-stress layer in which the logarithmic velocity-distribution law is valid, the eddy diffusivity $K_{M}$ is found from Eqs 115 and 187 to be

$$
\mathrm{K}_{\mathrm{M}}=\mathrm{u}_{*}^{2}\left(\frac{\partial \mathrm{u}}{\partial \mathrm{z}}\right)^{-1}=\mathrm{u}_{* \mathrm{kz}}=\mathrm{u}_{*} l
$$

where $l$ is the well-known "mixing length" of Prandtl Note that Eq 188 does not contain $z_{0}$ that arises as an integration constant This absence has important consequences in the interpretation of $z_{0}$ and for the extension of the logarithmic law to diabatic boundary layers 
Prandtl (1925) originally derived the logarithmic law on the basis of postulating the existence of a mixing length proportional to $\mathrm{z}$, but his arguments for the existence of a mixing length were flawed, and the idea of a physically significant mixing length was for many years discredited. Recently the concept of the mixing length has been on more rational foundations by associating it with a length scale of the turbulent motion. In the present context the mixing length arises in a formal way as the length scale of the eddy diffusivity.

The result of Eq. 1.88 gives some indication of the structure that the eddy viscosity must have. It is the product of a characteristic velocity-in this case the shear velocity - and a characteristic length of the turbulent motion. The characteristic length must express the size of the dominant eddies that contribute to the turbulent shearing stress. Such eddies are among the larger ones of the turbulent motion, and the mixing length $l=\mathrm{kz}$ expresses the notion that the larger eddies are about of the magnitude of the distance from the wall. All heuristic arguments leading to different forms of the eddy viscosity are essentially based on different conceptual models of the dynamics of turbulence, models yielding different characteristic lengths and velocities. For example, a turbulent wake with center velocity defect $u_{\max }$ and width $b$ at some distance $x$ from the origin has an eddy viscosity proportional to $u_{\max } \times b$ (Schlichting, 1968); the outer part of the turbulent boundary layer of thickness $h$ forming along a flat plate has an eddy viscosity proportional to $u_{\infty} \times h$ (Clauser, 1954), where $u_{\infty}$ is the velocity outside the boundary layer. The difficulty in specifying an eddy viscosity lies in the fact that the eddy viscosity depends un the development history of the turbulence structure. Only if the local turbulence structure develops in the same manner as the mean flow can a valid eddy viscosity be formulated which depends on local parameters only. This fact will become clearer when we consider the structure of turbulence in disturbed boundary layers.

In the derivation of velocity-distribution laws for the whole of the planetary boundary layer, it has been customary to define a suitable reference length $l$ which has the asymptotic behavior $l \sim \mathrm{z}$ for $\mathrm{z} \rightarrow 0$ and $l \propto \mathrm{G} / \mathrm{f}\left(\right.$ or $\mathrm{u}_{*} / \mathrm{f}$ ) at the edge of the outer layer because only in this manner can the dimensional form be correct. A formula that has this behavior has been given by Blackadar (1962) to

$$
l=\frac{\mathrm{kz}}{1+(\mathrm{kz} / \lambda)}
$$

where $\lambda$ is the mixing length at the outer edge:

$$
\lambda=0.00027 \frac{\mathrm{G}}{\mathrm{f}}
$$

The value of $\lambda$ is determined from a single profile and could be equally well recast into the form $\lambda \sim u_{*}$ /f required by the similarity theory. A close solution of Eqs. 1.36 and 1.37 with Eq. 1.89 is not available, and numerical techniques are required to predict velocity profiles. Closed solutions can be found, however, if in generalization of the 
eddy-diffusivity concept the angle between $\tau$ and $\mathbf{A}$ is specified as a function of $z$ Lettau (1970) has shown that, if this angle is constant, then the angle $\alpha_{0}$ determines the distribution law for eddy viscosity, $\alpha_{0}=45^{\circ}$ yielding the laminar constant-eddyviscosity solution In the same article, Lettau explored the possibility of empirically treating the tangent of the angle between $\tau$ and $\mathbf{A}$ as a linear function of height $\mathrm{He}$ claims that this dependency yields results in better agreement with observation than any of the constant-angle assumptions

\section{MODELING THE PLANETARY BOUNDARY LAYER}

One of two methods has customarly been followed in modeling the planetary boundary layer The first method relies on obtannng, in a strictly trial-and-error manner, a velocity profile that is similar in the laboratory and in the field over a significant fraction of the boundary-layer height The second method depends on using only that portion of a thick, naturally developed boundary layer in a wind tunnel that corresponds to the atmospheric logarithmic layer Whereas the first method has the advantage of giving thick boundary layers at short fetches and thus requires comparatively short wind-tunnel test sections, the latter is theoretically more sound in that it enables the establishment of exact similarity not only of the mean velocities but also of the turbulence structure Its disadvantage is, however, that a large test section is required for obtaining only small useful boundary-layer thicknesses Only the inner $15 \%$ of the boundary layer along a flat plate is logarithmic and thus represents the matched layer of the modeled atmospheric boundary layer

\section{The Power Law}

Of particular advantage in modeling by the first method is the use of the power law Already in the earliest studies of turbulent boundary layers, it was found by Blasius (see Schlichting, 1968) and others that the mean velocity distribution in the whole turbulent boundary layer could well be described by the empirical law

$$
\frac{\bar{u}}{u_{h}}=\left(\frac{z}{h}\right)^{1 / n}
$$

where $u_{h}$ is the velocity above the thickness $h$ of the boundary layer and $n$ is an exponent that depends, for aerodynamically smooth surfaces, on the Reynolds number and, for rough surfaces, on the type of roughness

The empirical power law, Eq 191, has two significant characteristics that make it very useful for work involving the whole of the layer $h$ The law is a good average representation of the velocity profile over the whole boundary layer, and integral relations based on this easily integrated law are not far from correct For those reasons 
the law has found wide acceptance also in meteorological work In particular, this law has often been applied to practical problems, such as predicting diffusion characteristics (Calder, 1949) or wind-tunnel modeling of wind forces (Davenport, 1965) It is a simple matter to plot experimental data on double logarithmic paper, and a purely empirical analysis of such plots for different types of terrain reveals the situation that 1s shown in Fig 112 (from Davenport, 1965) The rougher the terrain (1 e, the larger the obstructions on the surface which oppose the flow of the wind), the thicker will be the affected layer of air, and the more gradual will be the increase of velocity with height, as is reflected in the increase of the exponent $n$ with decreasing roughness Quantitatively, Davenport (1960) found the results shown in $F_{1 g} 11$ 13, where the thickness $h$ and the exponent $1 / \mathrm{n}$ are given as functions of the roughness of the ground The roughness is described by the roughness length $z_{0}$, obtained by fitting the logarithmic law to the velocity distribution near the ground Figure 113 makes it possible to estimate wind profiles by selecting a suitable roughness height and determining the coefficients of the profile from the graph The technique works quite well at high geostrophic winds, which are of particular interest for determining extreme wind loads on buldings, and the simplicity of the method makes it often well worthwhile to use it instead of the more elaborate procedure of Ekman layer determination outlined in the section titled Velocity Distribution near the Ground

The power law is also useful in guiding efforts to artificially create thick-boundarylayer velocity profiles in the laboratory which would allow the study of wind effects on relatively large-size models in wind tunnels with short test sections This can be done by introducing grids and other disturbances into the flow near the test-section entrance Typical attempts along this line have been reported by Lloyd (1967) and Counihan (1969) among many others These methods do not necessarly increase the thickness of the constant-stress layer Apparently an increase in free-stream turbulence level might be more beneficial for this purpose, but the experimental evidence is at present not conclusive

The power law may be used to determine which conditions must exist in the laboratory to satisfy the shear-stress distribution of the planetary boundary layer and, at the same time, to illustrate the effect of external boundary conditions on the eddy diffusivity In the laboratory the boundary-layer equations must be used without the Coriols forces

$$
\overline{\mathrm{u}} \frac{\partial \overline{\mathrm{u}}}{\partial \mathrm{x}}+\overline{\mathrm{w}} \frac{\partial \overline{\mathrm{u}}}{\partial \mathrm{z}}=-\frac{1}{\rho} \frac{\mathrm{d} \overline{\mathrm{p}}}{\mathrm{dx}}+\frac{1}{\rho} \frac{\partial \tau_{\mathrm{x}}}{\partial \mathrm{z}}, \frac{\partial \overline{\mathrm{u}}}{\partial \mathrm{x}}+\frac{\partial \overline{\mathrm{w}}}{\partial \mathrm{z}}=0
$$

from which we will infer the shear-stress distribution for two different simple cases a constant-thickness layer and a constant-pressure layer

When the layer, in the absence of Coriolis or other body forces, is of constant thickness, then a pressure gradient must exist which just compensates the flux of momentum to the ground This pressure gradient is impressed by conditions outside the boundary layer, where the shear stress is assumed to be zero, so that the pressure gradient and the reference velocity are related by Bernoulli's equation 


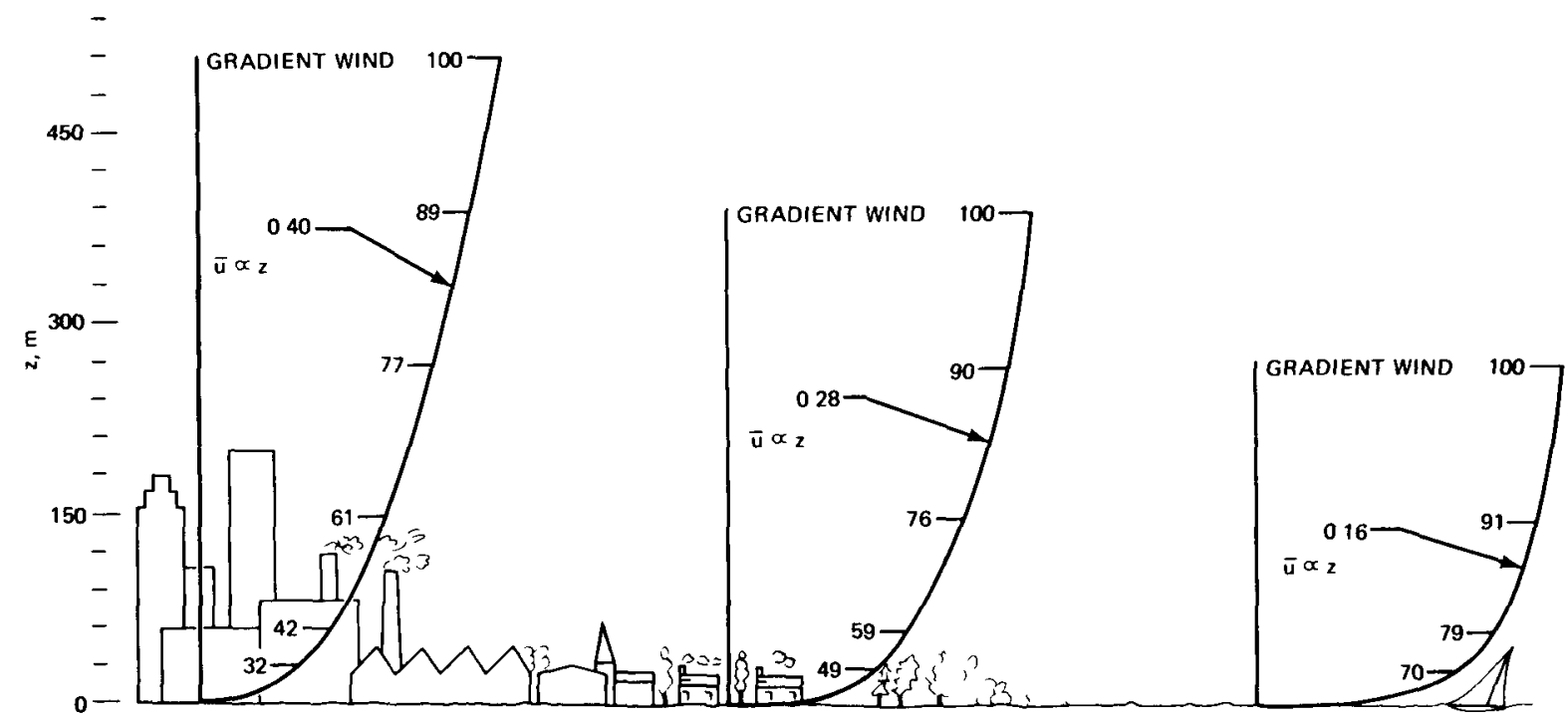

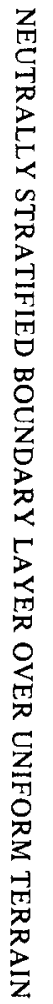

Fig. 1.12 Empincal power laws over different terrain, after Davenport (1965). 




Fig. 1.13 The exponent in the power law and the height of the boundary layer as functions of the roughness length and the type of terrain. From Davenport (1965).

$$
\frac{d p}{d x}=-\rho u_{\infty} \frac{d u_{\infty}}{d x}
$$

The pressure gradient is related to the ground shearing stress $\tau_{0}$ through Karman's integral equation

$$
\mathrm{u}_{*}^{2}=\frac{\tau_{0}}{\rho}=\frac{\mathrm{d}}{\mathrm{dx}}\left(\mathrm{u}_{\infty}^{2} \delta_{2}\right)+\delta_{1} \mathrm{u}_{\infty} \frac{\mathrm{du}_{\infty}}{\mathrm{dx}}
$$

which is obtained by integrating Eq. 1.92 over the thickness $h$ of the boundary layer. In this equation, $\delta_{2}$ and $\delta_{1}$ are the momentum and displacement thicknesses, respectively, which, for a power-law velocity prof 1 le, become 


$$
\delta_{2}=\int_{0}^{\mathrm{h}} \frac{\mathrm{u}}{\mathrm{u}_{\infty}}\left(1-\frac{\mathrm{u}}{\mathrm{u}_{\infty}}\right) \mathrm{dz}=\frac{\mathrm{n}}{(1+\mathrm{n})(2+\mathrm{n})} \mathrm{h}
$$

and

$$
\delta_{1}=\int_{0}^{h}\left(1-\frac{u}{u_{\infty}}\right) d z=\frac{1}{1+n} h
$$

For a constant-thickness layer, we find that the momentum equation becomes

$$
\tau_{0}=\rho\left(\delta_{2}+\frac{1}{2} \delta_{1}\right) \frac{\mathrm{du}_{\infty}^{2}}{\mathrm{dx}}=-\frac{2+3 \mathrm{n}}{(1+\mathrm{n})(2+\mathrm{n})} \mathrm{h} \frac{\mathrm{dp}}{\mathrm{dx}}
$$

where $\tau_{0}$ is the shear stress at the ground. Typically, $n=7$, so that

$$
-\frac{\mathrm{dp}}{\mathrm{dx}}=3.14 \frac{\tau_{0}}{\mathrm{~h}}
$$

The shear-stress distribution is obtained from Eqs. 1.92 and 1.91:

$$
\tau=\tau_{0}\left\{1-\frac{(1+n)(2+n)}{2+3 n} \frac{z}{h}\left[1-\frac{n^{2}}{(n+1)(2+n)}\left(\frac{z}{h}\right)^{2 / n}\right]\right\}
$$

The shear-stress distribution is shown for $n=7$ in Fig. 1.14. The distribution was nondimensionalized by $\tau_{0}$ and by the height $h$.

If the pressure is constant, Eq. 1.94 becomes for a power law

$$
u_{*}^{2}=u_{\infty}^{2} \frac{d \delta_{2}}{d x}=\frac{n}{(n+1)(n+2)} u_{\infty}^{2} \frac{d h}{d x}
$$

corresponding, for $n=7$, to an increase in boundary-layer thickness of

$$
\frac{\mathrm{dh}}{\mathrm{dx}}=10.3 \frac{\mathrm{u}_{*}^{2}}{\mathrm{u}_{\infty}^{2}}
$$

The shear-stress distribution obtained from Eq. 1.99 for the zero-pressure-gradient layer is given by

$$
\tau=\tau_{0}\left[1-\left(\frac{\mathrm{z}}{\mathrm{h}}\right)^{2 / \mathrm{n}+1}\right]
$$

which has also been shown in Fig. 1.14 for $n=7$. 


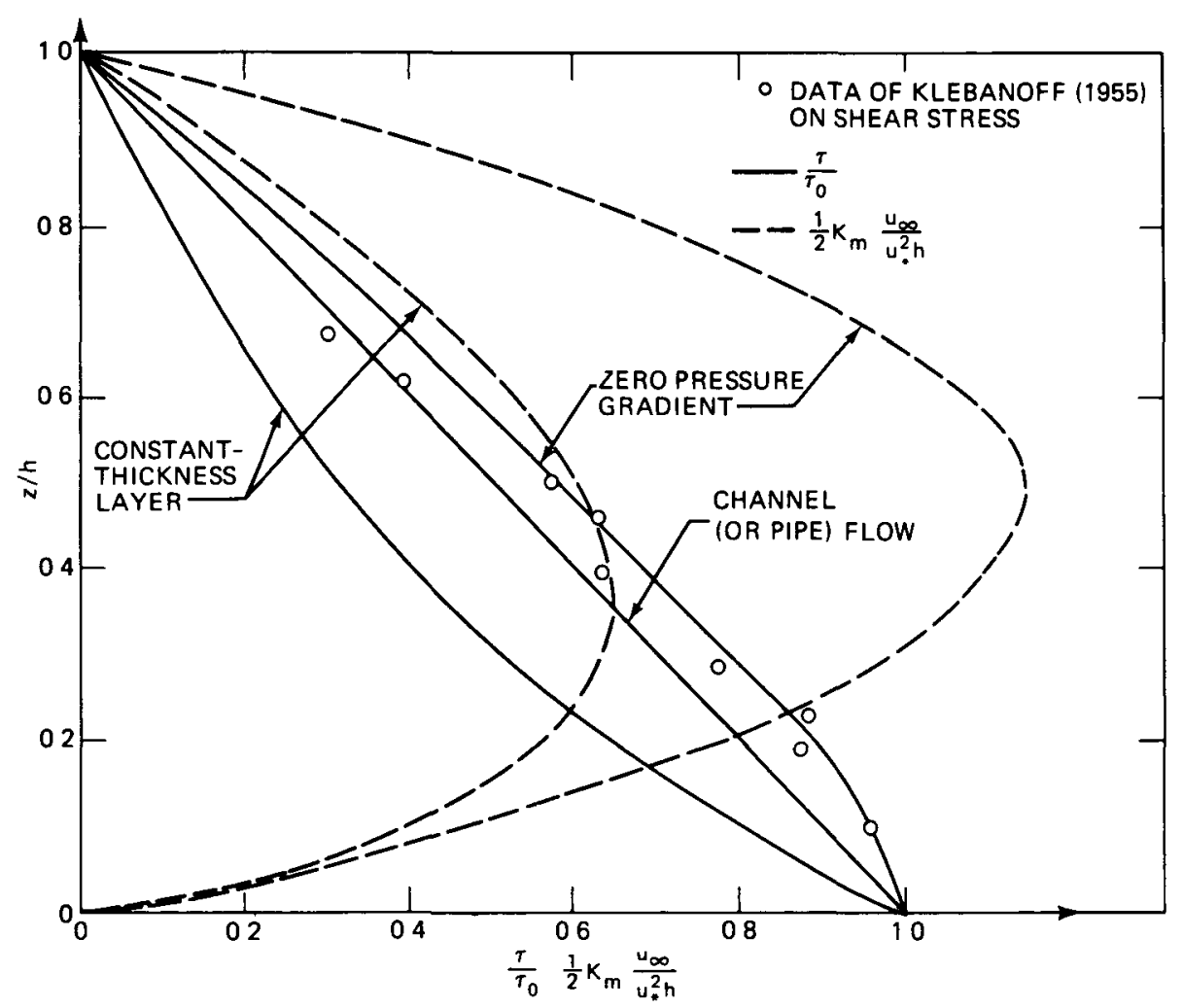

Fig. 1.14 Calculated shear-stress distributions and exchange coefficients in two different types of boundary layers which are described by the one-seventh power law, and comparison with data of Klebanoff (1955).

Note that the velocity distributions are identical in both cases of Fig 114 , and yet there is a very large difference in shear-stress distribution Consequently the distribution of eddy diffusivity $\mathrm{K}_{\mathrm{M}}$ is quite different for both cases Nondimensional eddy-diffusivity proflles are shown in Fig 114 as dashed curves We conclude that there cannot be a one to-one correspondence between velocity distribution and shear stress, a fact that must be taken into account in all sound models for a shear-stress distribution

An assumption in the data of $F_{1} g 114$ is that, as observations indicate, the exponent of the power law does not depend on $x$ An indirect confirmation of the validity of this assumption is obtained by comparing the calculated profiles with shear-stress distributions that were measured in a wind tunnel under constant-pressure conditions by Klebanoff (1955) Klebanoff's data have been plotted in Fig 114 and are seen to agree very well with the calculations except at the outer edge of the layer

An interesting observation is that, for a zero-pressure-gradient case, the shear-stress distribution shows a very small change of shear stress with distance from the wall In 
the lowest $15 \%$ of the boundary layer, the shear stress varies by less than $10 \%$. In contrast, the constant-thickness layer shows a shear-stress decrease of about $30 \%$ in the same region. The strong shear-stress gradients in the case of a constant-thickness layer have led Lettau (1959) to suggest that the atmospheric surface layer, because it is a constant-thickness layer and has a linear shear-stress distribution, be modeled in the laboratory by pipe or channel flow where the shear stress is also linearly distributed, with its maximum at the wall and a value of zero in the center of the pipe or conduit. The disadvantages of modeling the atmospheric surface layer in a conduit are associated with the effect of the walls on pressure distributions around models, or, in the case of studies of exchange processes, the different boundary conditions at the edge of the boundary layer which affect diffusion characteristics in all but the layers nearest the wall. Nevertheless, the analogy is a valid one, and its limitations need to be explored. Generally it is felt that the assumption of a constant layer is not critical in view of the small change in thickness required to maintain a typical wall shear stress. For example, a shear stress with a friction coefficient of $u_{*}^{2} / u_{\infty}^{2}$ of about 0.0025 is typical, and under these conditions the growth rate $\mathrm{dh} / \mathrm{dx}$ of the boundary layer is about $2.5 \%$.

\section{The Logarithmic Law}

Although valid only over the lower $15 \%$ of the boundary layer along a flat plate, the logarithmic layer in which the velocity distribution is expressed by

$$
\frac{\mathrm{u}}{\mathrm{u}_{*}}=\frac{1}{\mathrm{k}} \ln \frac{\mathrm{z}}{\mathrm{z}_{0}}
$$

is the only portion of the laboratory boundary layer which is the exact counterpart to the corresponding sublayer of the planetary boundary layer. In fact, the simplest way of deriving the logarithmic law with the fewest assumptions is by using arguments identical to the one used in the subsection Dimensional Considerations and the Inner Law of the Planetary Boundary Layer, except that the boundary-layer thickness $h$ and the Reynolds number replace the layer thickness $h$ and the Rossby number. Originally the logarithmic law had been derived by von Karman on the basis of arguments on local similarity of the profiles and shortly thereafter by Prandtl, who used the concept of the mixing length (Schlichting, 1968).

Within the logarithmic sublayer, modeling of the atmosphere then means exact scaling of the logarithmic law, and the effect of Coriolis forces can be neglected. Scaling requires that $u_{*}$ be the same in field and laboratory and that $z_{c} / z_{0}$ be constant in model and prototype, where $z_{0}$ is the roughness height and $z_{c}$ is a scaling length, such as the dimension of a building or of a terrain feature. An additional requirement is that

$$
\frac{\mathrm{u}_{*} \mathrm{z}_{0}}{\nu}>70
$$


to ensure independence of the flow from the Reynolds number. However, since changes of $\mathbf{u}_{*}$, for example, with Reynolds number, are small, this requirement can be relaxed if mean velocity profiles only are to be considered.

Strictly speaking, the model and prototype cases will differ in the distribution of shear stress, which varies slightly with height, and at a different rate in a zero-pressure-gradient boundary layer as compared with the planetary boundary layer. A more refined technique is theoretically possible, by delicately adjusting the wind-tunnel pressure gradient until the laboratory shear stress has exactly the same scaled distribution as the atmospheric boundary layer. Usually, however, such refinements are not justified either by the accuracy of field observations or by the gain in agreement between model and prototype results, particularly since the logarithmic law is valid only in the lower $15 \%$ of the boundary layer in which the shear stress deviates not much more than $10 \%$ from its value at the ground.

The atmospheric modeling does not exhaust the possibilities of wind-tunnel applications to the study of the atmospheric surface layer. A number of basic studies are needed to fill the gaps in our knowledge of the neutrally stratified boundary layer. Important contributions could be made by fundamental investigations of the nature of aerodynamic roughness, although this problem has defied previous at tempts. Ideally, it should be possible to infer the aerodynamical behavior of a surface from the geometry and perhaps the elasticity of the roughness elements of the surface, but it is not easy to construct a model of the roughness effect on that basis alone. More fruitful are experiments on typical configurations of the roughness elements for atmospheric boundary layers, such as the ones made by Chamberlain (1966), Hsi and Nath (1968), and Plate and Quraishi (1965).

\section{REFERENCES}

Allen, L. H., C. S. Yokum, and E. R. Lemon, 1964, Photosynthesis Under Field Conditions: Pt. VII: Radiant Energy Exchange Within a Corn Crop and Implications in Water Use Efficiency, Agron. J., 56: 253-259.

Batchelor, G. K., 1967, An Introduction to Fluid Dynamics, Cambridge University Press, Cambridge, England.

Blackadar, A. K., 1962, The Vertical Distribution of Wind and Turbulent Exchange in a Neutral Atmosphere, J. Geophys. Res., 67: 3095-3102.

, and H. Tennekes, 1968, Asymptotic Similarity in Neutral Barotropic Planetary Boundary Layers, J. Atmos. Sci., 25: 1015-1020.

Calder, K. L., 1949, Eddy Diffusion and Evaporation in Flow over Aerodynamically Smooth and Rough Surfaces: a Treatment Based on Laboratory Laws of Turbulent Flow with Special Reference to Conditions in the Lower Atmosphere, Quart. J. Mech. Appl. Math., 2: 153-176.

Chamberlain, A. C., 1966, Transport of Gases to and from Grass and Grass-Like Surfaces, Proc. Roy. Soc. (London), Ser, A, 290: 236-265.

Cionco, R. M., 1965, A Mathematical Model for Air Flow in a Vegetative Canopy, J. Appl Meteorol., 4: 515-522.

Clarke, R. H., 1969, Observational Studies in the Atmosphere Boundary Layer, Quart. J. Roy. Meteorol. Soc., 96: 91-114. 
Clauser, F. H., 1954, Turbulent Boundary Layers in Adverse Pressure Gradients, J. Aeronaut. Sci., 21: 91-108.

Counihan, C., 1969, An Improved Method of Simulating an Atmospheric Boundary Layer in a Wind Tunnel, in Atmos. Environ., 3: 197-214, Pergamon Press, Inc.

Csanady, G. T., 1967, On the Resistance Law of a Turbulent Ekman Layer, J. Atmos. Sci., 24: 467-471.

Davenport, A. G., 1960, A Rationale for the Determination of Design Wind Velocities, Proc. Amer. Soc. Civil Eng., J. Struct. Div., 86: 39.

_- 1965, The Relationship of Wind Structure to Wind Loading, National Physical Laboratory, Symposium No. 16, Wind Effects on Buildings and Structures, pp. 54-102, Her Majesty's Stationery Office, London.

De Marrais, G. A., 1959, Wind Speed Profiles at Brookhaven National Laboratory, J. Meteorol., 16: 181-190.

Deacon, E. L., 1953, Vertical Profiles of Mean Wind in the Surface Layers of the Atmosphere, Geophysical Memorandum No. 91, London, Air Ministry: Metropolitan Office.

Deardorff, J., personal communication, 1969.

Hama, F. H., 1954, Boundary Layer Characteristics for Smooth and Rough Surfaces, Soc. Naval Architects Marine Eng., Trans., 62: 333-358.

Hinze, J., 1959, Turbulence, McGraw-Hill Book Company, Inc., New York.

Hsi, G., and J. H. Nath, 1968, Wind Drag Within a Simulated Forest Canopy Field, Technical Report No. CER 68-69GH-JHN6, prepared for U.S. Army Material Command, by Fluid Dynamics and Diffusion Laboratory, Colorado State University, 127 pp.

Johnson, W. B., 1965, Atmospher1c Boundary Layer Dynamics Over the Forests of Northeastern Wisconsin, in Final Report, Studies of the Effects of Variations in Boundary Conditions on the Atmospheric Boundary Layer, Department of Meteorology, University of Wisconsin.

Johnson, O., 1959, An Examination of the Vertical Wind Profile in the Lowest Layers of the Atmosphere, J. Meteorol., 16:144-148.

Kasanski, A. B., and A. S. Monin, 1960, The Turbulent Regime Above the Surface Atmospheric Layer, Bull. Acad. Sci. USSR, Geophys. Ser. (Engl. Transl.), No. 1.

Klebanoff, P. S., 1955, Characteristics of Turbulence in a Boundary Layer with Zero Pressure Gradient, National Advisory Committee for Aeronautics, Annual Report No. 1247, 19 pages.

Kung, E. C., 1961, Derivation of Roughness Parameters from Wind-Profile Data over Tall Vegetation, in Report on Studies of the Three-Dimensional Nature of the Planetary Boundary Layer, Department of Meteorology, University of Wisconsin.

Lemon, E. R., 1963, The Energy Budget of the Earth's Surface, Part II. Production Research Report No. 2, Agriculture Research Service, U. S. Department of Agriculture, $49 \mathrm{pp}$.

Lettau, H. H., 1950, A Re-examination of the 'Leipzig Wind Profile', Tellus, 2: 125-129.

__, 1957, Windprofil, innere Reibung und Energieumsatz in den unteren $500 \mathrm{~m}$ überm Meer, Beitr. Phys. Atmos., 30: 78-96.

_., 1959, Wind Profile, Surface Stress and Geostrophic Drag Coefficients in the Atmospheric Surface Layer, in Advances in Geophysics, Vol. 6, Academic Press, Inc., pp. 241-257.

_, 1962, Theoretical Wind Spirals in the Boundary Layer of a Barotropic Atmosphere, Beitr. Phys. Atmos., 36: 195-212.

__, and H. Hoeber, 1964, Über die Bestimmung der Höhenverteilung von Schubspannung und Austrauschkoeffizient in der Atmosphärischen Reibungsschicht, Beitr. Phys. Atmos., 37: 105-118.

_, 1969, Note on Aerodynamic Roughness Parameter Estimations on the Basis of Roughness Element Description, J. Appl. Meteorol., 8: 828-832. 1970, Variangular Wind Spirals, in Boundary Layer Meteorol., 1: 64-79.

Lloyd, A., 1967, The Generation of Shear Flow in a Wind Tunnel, Quart. J. Roy. Met. Soc., 93: $79-96$.

Lumley, T. L. and H. A. Panofsky, 1964, The Structure of Atmospheric Turbulence, Wiley-Interscience, Inc., $239 \mathrm{pp}$. 
Malkus, M. V. R., 1956, Outline of a Theory of Turbulent Shear Flow, J. Fluid Mech., 1: 521-540. Paeschke, W., 1937, Experimentelle Untersuchungen zum Rauhigkeits-und Stabilitaets-problem in der freien Atmosphaere, Beitr. Phys. Atmos., 24: 163-189.

Prandtl, L., 1965, Führer durch die Strömungslehre, 6th ed., Vieweg Brauschweig, Germany.

- 1925, Uber die ausgebildete Turbulenz, Z. angew. Math. Mech., (ZAMM), 5: 136-139.

Plate, E., P. C. Chang, and G. M. Hidy, 1969, Experiments on the Generation of Small Water Waves by Wind, J. Fluid Mech., 35: 625-656.

Plate, E., and A. A. Quraishi, 1965, Modeling of Velocity Distribution Inside and Above Tall Crops, J. Appl. Meteorol., 4: 400-408.

Priestley, C. H. B., 1959, Turbulent Transfer in the Lower Atmosphere, The University of Chicago Press, Chicago.

Reynolds, W. C., and W. G. Tiederman, 1967, Stability of Turbulent Channel Flow, with Application to Malkus' Theory, J. Fluid Mech., 27: 253-272.

Roll, H. U., 1965, The Physics of the Marine Atmosphere. Academic Press, Inc., New York.

Rossby, C. G., and R. B. Montgomery, 1935, The Layers of Frictional Influence in Wind and Ocean Currents, Pap. Phys. Oceanogr. Meteorol., 3(3): 101 pp.

Sandborn, V. A., 1959, Measurement of Intermittency of Turbulent Motion in a Boundary Layer, J. Fluid Mech., 6: 221-240.

Sayre, W. W., and M. L. Albertson, 1963, Roughness Spacing in Rigid Open Channels, Trans. Amer. Soc. Civil Engrs., 128(Part 1): 343-427.

Schlichting, H., 1968, Boundary Layer Theory, McGraw-Hill Book Company, Inc., New York.

Slotta, L. S., 1963, A Critical Investigation of the Universality of Karman's Constant in Turbulent Flow, in H. Lettau, Annual Report, 1963, Department of Meteorology, University of Wisconsin, to Department of Meteorology, U. S. Army Research and Development Activity, Ft. Huachuca, Ariz.

Stoller, T., and E. R. Lemon, 1963: see Lemon, 1963.

Swinbank, W. C., 1969, Structure of Wind and the Shearing Stress in the Planetary Boundary Layer, to be published.

-, and A. J. Dyer, 1967, An Expenmental Study in Micrometeorology, Quart. J. Roy. Met. Soc., 93: 495-500.

Tan, H. S., and S. C. Ling, 1967: reprinted in Lemon, 1963.

Tanner, C. B., and W. L. Pelton, 1960, Potential Evapotranspiration Estimates by the Approximate Energy Balance Method of Penman, J. Geophys. Res., 65: 3391-3413.

Townsend, A. A., 1956, The Structure of Turbulent Shear Flows, Cambridge University Press, Cambridge, England.

Uchijima, Z., and J. L. Wright, 1964, An Experimental Study of Airflow in a Corn-Plant Layer, Bull. Nat. Inst. Agric. Sci. Japan, Ser. A, 11: 19-65.

van Dyke, M., 1964, Perturbation Methods in Fluid Mechanics, Academic Press, Inc., New York.

Weiler, H. S., 1969, Prediction of Vertical Profiles of Wind Speed over a Lake, Proceedings of $12 \mathrm{th}$ Conference on Great Lakes Research, International Association for Great Lakes Research, pp. 492-503.

Wieghardt, K., 1944, Uber die turbulente Stromung im Rohr und längs der Platte, Z. angew Math. Mech. (ZAMM), 24: 294-306.

Zilitinkevich, S. S., D. L. Laikhtman, and A. S. Monin, 1967, Dynamics of the Atmospheric Boundary Layer (in Russian), Izv. Akad, Nauk SSSR Fiz. Atmos, Okeana, 3: 297-333 (English transl., pp. 170-191). 
- 


\section{THE STRATIFIED ATMOSPHERIC BOUNDARY LAYER NEAR THE GROUND}

The diurnal changes in solar radiation set up a cycle of cooling and heating of the planetary boundary layer which is strongly reflected in the wind field. Early in the morning, before sunrise, the stratification of the air layer is stable because the ground is cooler than the air masses above. With the rising of the sun, on a clear day, solar radiation makes the ground heat up much faster than the overlying air. When the ground is warmer than the air, heat moves from it to the air in contact with the ground. The warm air expands and becomes lighter than the air above it. Parcels of heated air tend to move up into the cooler air above while cooler air moves down, i.e., the air layer is unstable. If the air could adjust instantaneously to the buoyancy that is generated by the heating, a layer of constant (potential) temperature equal to that at the ground would be maintained at all times. Actually, however, the heating of the air lags behind the heating of the ground. Clear and dry air over dry ground obtains almost all its heat from the ground. Since directly at the ground no air velocities can exist, the transfer of heat from the ground takes place by molecular conduction, down a very strong temperature gradient. The molecular-conduction layer is very thin and changes into the forced-convection layer in which heat is transported by a turbulence that is almost entirely caused by mechanical, i.e., shear-stress, action. At larger distances from the ground, turbulent shear stresses become progressively weaker and gradients in both temperature and velocity decrease. The temperature distribution remains superadiabatic; but with increasing heights less turbulence is maintained by shear stresses, and an increasing amount of turbulence is buoyancy induced. The forced-convection layer gradually changes into a free-convection layer. Out of the 
superadiabatic layer, turbulent parcels of warmer air rise into colder regions aloft and mix with the surroundings in the free-convection layer, which is vigorously agitated because the warm thermal plumes or thermals have significant vertical uplift velocities, and the mass transport associated with them creates a downward motion of colder, but also turbulent, air. The result is a mixing of the air which is so strong that in the free-convection layer the (potential) temperature is almost constant (Webb, 1958).

The layer in which the temperature field is influenced by the heat flux from the ground is the convective layer. It is capped by an inversion, 1 e., by the stably stratified air aloft, which begins near the elevation where the undisturbed stable potential temperature is equal to the mean potential temperature of the convective layer. In the course of the day, when the ground gets warmer and the alr above it heats up, the convective layer increases in thickness, at a rate that is determined by the heat flux from the ground, which in turn depends on the temperature gradient across the superadiabatic layer.

Late in the afternoon an instant of quasi-equilibrium is reached, and the ground is at nearly the same temperature as the layer above it Since there are then almost no gradients in temperature at the ground, the conductive heat flow from the ground ceases to exist, and in the absence of fluxes all temperature fluctuations must die out, so that the neutral stratified planetary boundary layer is asymptotically free of fluctuating temperature everywhere. It is this condition to which the considerations of the previous chapter apply, and it is readily apparent that it is a rather exceptional state of the atmosphere.

Later in the day the temperature of the arr exceeds that of the ground, and the ensuing heat flux down to the ground results in a stably stratified temperature-gradient layer near the ground and causes a gradual cooling of the convective layer. The herght at which the upper inversion occurs is lowered, while a ground-based inversion builds up, particularly after the sun sets. If the conditions for cooling of the surface persist long enough, then eventually the upper and lower inversions destroy the constanttemperature layer between them and join, and further cooling steepens the stable temperature gradient until the next morning when the cycle starts again.

The stable boundary layer at the ground bulds up in time in the same manner as the unstable superadiabatic layer. But the thicker the stable layer gets the more the turbulent energy balance in its upper part is dominated by the heat flux toward the ground, and there exists a critical condition, corresponding to a critical flux Ruchardson number of the local flow at which turbulence suddenly becomes almost completely damped out by the buoyancy effect, so that the stable surface layer and the remnants of the convective layer aloft become uncoupled because the eddy viscosity drops to near the value of the molecular viscosity. The stable surface layer is very quickly retarded in a matter of minutes (Townsend, 1967) by the action of the friction on the ground which is transmitted to the decoupled lower layer by the turbulent shear stresses that exist in 1 , whle for the same reason the upper layer accelerates. After a very short time, the velocity gradient everywhere in the lower layer is reduced so much that the flux Richardson number increases above its critical value and turbulence is suppressed everywhere the flow approaches laminar flow in the 
lower layer except near the interface above. Since across the interface between the two layers the shear stress must be contınuous, it follows that

$$
\tau=\left.\epsilon_{\mathrm{u}} \frac{\partial \overline{\mathrm{u}}}{\partial \mathrm{z}}\right|_{\mathrm{u}}=\left.\epsilon_{l} \frac{\partial \overline{\mathrm{u}}}{\partial \mathrm{z}}\right|_{l}
$$

where the subscripts $\mathrm{u}$ and $l$ refer to conditions below and above the interface, respectively, and $\overline{\mathrm{u}}$ is the mean velocity The eddy viscosity $\epsilon_{\mathrm{u}}$ is near the molecular value, $\epsilon_{l}$ is therefore much larger, and a strong gradient develops near the interface in the lower fluid which lowers the Richardson number and thus permits turbulence to exist at the interface. Too much turbulence would reduce the gradient again, therefore the turbulence is likely to be weak It is sufficiently strong, however, to mix the stable layer below the interface with the convection layer above, thus providing a mechanism by which the stable layer can thicken in time

With relaminarization of the lower layer, its coupling to the ground is greatly reduced, and the effect of the shear stress on top of the lower layer together with the Coriolis force leads to an acceleration of the flow in the lower layer But, since only molecular viscosity is acting near the top of the lower layer, the acceleration leads to a very slow increase of the velocity which reaches geostrophic magnitudes only after hours When in the course of this development the gradient Richardson number falls below a critical value either because of changes in heat flux or because of the increase in velocity gradient, then the lower layer becomes turbulent again and is quickly retarded in the same manner as before, unt1l the heat flux reverses due to changes in surface temperature after sunrise.

The very complex mechanism outlined in the previous paragraphs provides the reason why planetary-boundary-layer models of the kind considered in the first chapter are not likely to be applicable to the stratified atmosphere unless in unstable conditions the convection layer is so thick that the whole of the Ekman spiral is contained in it When the convection layer is thinner than the Ekman layer, it is necessary that analytical models describing the boundary layer take account of the change in turbulence characteristics across the interface between convection layer and outer, stable region. As will become more evident in Chap. 3, the structure of turbulence near the interface is far from well known, but it is almost certainly not possible to neglect the temporal development of the convection layer. Steady-state models fail to adequately predict the experimental parameters of Ekman spirals observed in the lower atmosphere (as is evident, for example, in the collection of data reported by Zilitınkevich et al , 1967), and no reasonable models exist at this time to quantitatively describe the stratıfied planetary boundary layer There exists, however, in stratified planetary boundary layers also an inner layer, corresponding to that observed under neutral conditions in which Conolis forces have negligible effects This is the layer in which ground shear stress dominates in determining the flow conditions The dynamic conditions in this stably or unstably stratified layer near the ground will be discussed in this chapter, and all Coriolis effects will be neglected. 


\section{BASIC EQUATIONS FOR SLIGHTLY STRATIFIED FLUIDS}

\section{The Conservation Equations}

The analytical treatment of the thermally stratified planetary boundary layer is severely limited by the complexities of the basic field equations. To the Navier-Stokes equations for turbulent fluids, which were discussed in Chap. 1, one must add the conservation equation for the energy as well as an equation of state and a set of constituent equations for the dependency of the fluid properties on temperature and pressure. The most important effect of an addition of heat to the fluid is, however, the buoyancy effect induced by the density stratification of a fluid with temperature gradients. Generally the density changes are small compared with the mean density, and they become effective in disturbing the fluid only when coupled with the acceleration of gravity, i.e., by inducing buoyancy forces.

In considering the basic equations of the diabatic planetary boundary layer, we shall first derive the general equations subject only to the restrictions of small density fluctuations. Ultimately we shall be interested here in only a fully developed layer of constant thickness which is heated or cooled uniformly from below, without considering a feedback between the ground temperature and the temperature of the fluid, i.e., the temperature of the ground is a constant independent of the downwind distance $\mathrm{x}$ that is given as part of the boundary conditions. We shall also restrict the discussion to a two-dimensional mean flow field for which Coriolis effects can be neglected. The z-axis is directed vertically upward from the ground, and the ground is assumed to be horizontal. For this situation the flow field is governed by the following set of equations:

The equation of continuity:

$$
\left(\frac{\partial}{\partial \mathrm{t}}+\mathbf{v} \cdot \nabla\right) \rho+\rho \nabla \cdot \mathbf{v}=0
$$

The momentum equation:

$$
\left(\frac{\partial}{\partial \mathrm{t}}+\mathbf{v} \cdot \nabla\right) \mathbf{v}=-\frac{1}{\rho} \nabla \mathrm{p}-\mathrm{gk}+\nu\left[\nabla^{2} \mathbf{v}+\frac{1}{3} \nabla(\nabla \cdot \mathbf{v})\right]
$$

The energy equation:

$$
\rho \mathrm{c}_{\mathrm{v}}\left(\frac{\partial}{\partial \mathrm{t}}+\mathrm{v} \cdot \nabla\right) \mathrm{T}+\mathrm{p} \nabla \cdot \mathrm{v}=\mathrm{k} \nabla^{2} \mathrm{~T}+\rho \phi+\rho \mathrm{c}_{\mathrm{p}} R
$$

The equation of state:

$$
p=\rho \mathbf{R T}
$$


In these equations $\mathbf{v}$ is the instantaneous velocity vector, $\mathrm{T}$ the instantaneous temperature, and $\rho$ and $\mathrm{p}$ the instantaneous density and pressure, respectively The quantity $\rho \phi$ in $\mathrm{Eq} 2.3$ is the instantaneous dissipation of kinetic energy into heat, and $\rho c_{p} R$ represents the heat added instantaneously by radiation. The thermal conductivity $k$, the specific heat at constant volume $c_{v}$, and the kinematic viscosity $v$ are all assumed to be independent of the temperature or density fluctuations and determined by some mean value.

The energy equation and the equation of state reflect the assumption that the air is an ideal gas in which the internal energy is equal to $c_{v} T$, where $T$ is the local temperature. These conditions are well satisfied in the atmosphere The basic equations can also be derived for more general types of fluids, in the same manner as shown here, and the results, under the same restrictions as used here, are found to be identical (Dutton and Fichtl, 1969)

Dry and clean air is transparent to thermal radiation, and the radiation term in the energy equation is important only when water vapor or pollutants, like $\mathrm{CO}_{2}$ or particulate matter, exist in the arr. Unless stated otherwise, we shall be concerned only with dry and pollutant-free air

\section{Hydrostatic Equations}

The simplest state of the atmosphere is its state of rest. When all velocity components are zero, Eq. 2.2 reduces to a balance between gravity and vertical pressure gradient

$$
\frac{\partial p}{\partial z}=-g \rho
$$

1 e., the pressure decreases with height from 1ts surface value. A pressure value in the atmosphere at rest [expressed in millibars $(\mathrm{mb})$ ] is therefore a measure of the altitude, and in meteorology the two are occasionally used interchangeably.

It is useful at this stage to also introduce the potential temperature $\theta$ for the atmosphere in hydrostatic equilibrium, which is defined as the temperature that a parcel of air, of temperature $T$ and pressure $p$ at its present level, would have if brought adiabatically to the $1000-\mathrm{mb}$ pressure level in the atmosphere at rest. Since, for an adiabatic process,

$$
\mathrm{T} \mathrm{p}^{-k}=\text { constant }
$$

where $k=R / c_{p}$ (see Fleagle and Businger, 1963), it follows that the potential temperature is defined to

$$
\mathrm{T} \cdot \mathrm{p}^{-\kappa}=\theta(1000 \mathrm{mb})^{-\kappa}
$$


The vertical gradient of potential temperature is found, by differentiating the logarithm of Eq 2.7, to

$$
\frac{1}{\theta} \frac{\partial \theta}{\partial \mathrm{z}}=\frac{1}{\mathrm{~T}} \frac{\partial \mathrm{T}}{\partial \mathrm{z}}-\frac{\mathrm{R}}{\mathrm{c}_{\mathrm{p}}} \frac{1}{\mathrm{p}} \frac{\partial \mathrm{p}}{\partial \mathrm{z}}
$$

Replacing $\partial \mathrm{p} / \partial \mathrm{z}$ by Eq. 2.5 and p by Eq. 2.4 yields, for the gradient of potential temperature,

$$
\frac{\partial \theta}{\partial z}=\frac{\theta}{T}\left(\frac{\partial T}{\partial z}+\frac{g}{c_{p}}\right) \approx \frac{\partial T}{\partial z}+\frac{g}{c_{p}}
$$

because the potential temperature is not much different from the actual temperature in total magnitude. The ratio $\mathrm{g} / \mathrm{c}_{\mathrm{p}}$ is the adiabatic lapse rate equal to about $0.01^{\circ} \mathrm{C} / \mathrm{m}$, which is the temperature gradient that would be found in an atmosphere at rest without heat input from the boundaries In an atmosphere where the temperature gradient balances the adiabatic lapse rate (1.e., where the potential temperature gradient is zero), an air particle that is moved (very slowly) around adiabatically always has the temperature of the surroundings and is thus in neutral equilibrium. In an atmosphere with a positive potential gradient, an air particle that is moved from a lower level adiabatically to a higher level would have a temperature below that of its surroundings, it would therefore be heavier and fall back to a lower level. If the same partıcle was displaced adiabatically downward, it would be lighter than its surroundings. Consequently the particle in its original position is in stable equilibrium. Conversely, in a negative potential temperature gradient, the particle displaced from its equilibrium level would tend to move further away from its original position, at its original position it therefore is in unstable equilibrium. An atmosphere is called unstable if it has a negative potential temperature gradient, neutral if it has a zero potential temperature, and stable if it has a positive potential temperature gradient.

\section{The Conservation Equations to the Boussinesq Approximation}

We shall now derive the equations of motion for deviations from a steady reference state. If the reference state changes only very little with height, it will be possible to greatly simplify Eqs. 2.1 to 2.3. This has first been shown by Boussinesq (1903) for an incompressible medium, such as water, in which density fluctuations arise from admixtures of fluids of different specific weights. Recently, however, it has been shown by Spiegel and Veronis (1960) that, for a shallow layer of fluid whose lateral scale is much larger than its vertical scale, the Boussinesq approximations can be derived without the restriction of incompressibility. This derivation was restated and somewhat elucidated by Calder (1968). Ogura and Phillips (1962) have shown that the assumptions of Spiegel and Veronis (1960) are compatible with all likely states of the atmosphere caused by small perturbations of the rest state except for those perturbations which are propagated by pressure fluctuations, 1.e., acoustic waves. 
These must propagate with infinite velocity, 1.e., they cannot exist. Finally, Dutton and Fichtl (1969) have shown that the Boussinesq approximation is not necessarily restricted to shallow layers.

In deriving the equations of motion to the Boussinesq approximations, we consider the set of Eqs. 2.1 to 24 for the motions induced by small perturbations of a reference state for a fluid at rest, 1.e., defined by

$$
\mathbf{v}_{\mathrm{R}}=0
$$

We also define the reference temperature condition as that of constant potential temperature, so that

$$
\frac{\partial T_{R}}{\partial z}=-\frac{g}{c_{p}}
$$

or

$$
\mathrm{T}_{\mathrm{R}}=\mathrm{T}_{0}\left(1-\frac{\mathrm{gz}}{\mathrm{c}_{\mathrm{p}} \mathrm{T}_{0}}\right)
$$

where $T_{0}$ is the ground temperature. Note that the assumption of a steady reference state was made, all time variations of the reference situation must be accounted for in the equations for the deviations from the reference state $A$ consequence is that the time average of the deviation from the mean is not zero It would, of course, be possible to consider, instead of the steady reference state, a time-dependent state, in which case the temperature profile would no longer be linear

The equations for the perturbations are obtained if the equations of the reference state are subtracted from Eqs 2.1 to 23 . To facilitate this operation, we find it useful to split the variables

Let the deviation of the density from the reference state locally be denoted by $\rho^{\prime}$ and the deviations of pressure and temperatures by $\mathrm{p}^{\prime}$ and $\mathrm{T}^{\prime}$, respectively, so that

$$
\begin{aligned}
& \rho=\rho_{\mathrm{R}}+\rho^{\prime}=\rho_{\mathrm{m}}+\Delta \rho+\rho^{\prime} \\
& \mathrm{p}-\mathrm{p}_{\mathrm{R}}+\mathrm{p}^{\prime}=\mathrm{p}_{\mathrm{m}}+\Delta \mathrm{p}+\mathrm{p}^{\prime} \\
& \mathrm{T}=\mathrm{T}_{\mathrm{R}}+\mathrm{T}^{\prime}=\mathrm{T}_{\mathrm{m}}+\Delta \mathrm{T}+\mathrm{T}^{\prime}
\end{aligned}
$$

where the subscript $m$ denotes mean values for the whole layer Now subtract the reference state from the equations of motion and energy

$$
\left(\frac{\partial}{\partial \mathrm{t}}+\mathbf{v} \cdot \nabla\right) \mathbf{v}=-\frac{1}{\rho} \nabla \mathrm{p}^{\prime}-\mathrm{g} \frac{\rho^{\prime}}{\rho} \mathbf{k}+\nu\left[\nabla^{2} \mathbf{v}+\frac{1}{3} \nabla(\nabla \cdot \mathbf{v})\right]
$$

and 


$$
\rho c_{\mathrm{v}}\left(\frac{\partial}{\partial \mathrm{t}}+\mathrm{v} \cdot \nabla\right) \mathrm{T}=-\mathrm{p} \nabla \cdot \mathrm{v}+\mathrm{k} \nabla^{2} \mathrm{~T}^{\prime}+\rho \phi+\rho \mathrm{c}_{\mathrm{p}} R
$$

The quantities $\phi$ and $R$ in Eq. 2.15 remain unchanged because there is nether dissipation nor radiation in the reference state. Now assume that the fluctuations in density as well as the deviation of the reference density from some constant mean value, $\rho_{\mathrm{m}}$, are small. Then the density can be expanded about the mean value in a Taylor series, and from the equation of state, Eq 2.4, one obtains

$$
\rho=\rho_{\mathrm{m}}\left(1+\frac{\Delta \mathrm{p}}{\mathrm{p}_{\mathrm{m}}}-\frac{\Delta \mathrm{T}}{\mathrm{T}_{\mathrm{m}}}\right)+\rho_{\mathrm{m}}\left(\frac{\mathrm{p}^{\prime}}{\mathrm{p}_{\mathrm{m}}}-\frac{\mathrm{T}^{\prime}}{\mathrm{T}_{\mathrm{m}}}\right)
$$

where the first term on the right is the reference density $\rho_{R}$ With this expression the vertical component of the first two terms on the right side of Eq. 214 becomes

$$
-\frac{1}{\rho} \frac{\partial \mathrm{p}^{\prime}}{\partial \mathrm{z}}-\mathrm{g} \frac{\rho^{\prime}}{\rho}=-\frac{1}{\rho} \frac{\partial \mathrm{p}^{\prime}}{\partial \mathrm{z}}\left(1+\frac{\mathrm{g} \rho_{\mathrm{m}}}{\mathrm{p}_{\mathrm{m}}} \frac{\mathrm{p}^{\prime}}{\frac{\partial \mathrm{p}^{\prime}}{\partial \mathrm{z}}}\right)+\mathrm{g} \frac{\rho_{\mathrm{m}}}{\mathrm{p}} \frac{\mathrm{T}^{\prime}}{\mathrm{T}_{\mathrm{m}}}
$$

Spregel and Veronis (1960) have argued that the length $\mathrm{p}_{\mathrm{m}} / \mathrm{g} \rho_{\mathrm{m}}=\mathrm{h}$ is very large, so that the ratio $p^{\prime} / h$ is much smaller than $\partial p^{\prime} / \partial z$ But since $p^{\prime}$ is caused by the fluctuating velocities, it does not appear possible to make assumptions on $\mathrm{p}^{\prime}$ or $\partial \mathrm{p}^{\prime} / \partial \mathrm{z}$ without considering the velocity field. Actually it can readily be shown that dropping the $p^{\prime}$ term implies an assumption on the ratio of $p^{\prime} / T^{\prime}$. For if both the last term of Eq 2.17 and $(1 / \rho)\left(\partial p^{\prime} / \partial z\right)$ are to be retained, then they must be terms of the same order, 1.e.,

$$
\frac{\partial \mathrm{p}^{\prime}}{\partial \mathrm{z}}=0\left(\mathrm{~g} \rho_{\mathrm{m}} \frac{\mathrm{T}^{\prime}}{\mathrm{T}_{\mathrm{m}}}\right)
$$

Consequently

$$
\frac{\mathrm{g} \rho_{\mathrm{m}}}{\mathrm{p}_{\mathrm{m}}} \frac{\mathrm{p}^{\prime}}{\frac{\partial \mathrm{p}^{\prime}}{\partial z}}=0\left(\frac{\mathrm{p}^{\prime}}{\mathrm{T}^{\prime}} \frac{\mathrm{T}_{\mathrm{m}}}{\mathrm{p}_{\mathrm{m}}}\right)
$$

and, if we consider this term small compared with 1 , then we are in effect assuming that, with Eqs. 2.13 and 2 16,

$$
\rho^{\prime}=-\rho_{\mathrm{m}} \frac{\mathrm{T}^{\prime}}{\mathrm{T}_{\mathrm{m}}}
$$

which we shall use henceforth

Consider now the contınuity equation, Eq 21 , which can be written 
BASIC EQUATIONS FOR SLIGHTLY STRATIFIED FLUIDS

$$
\rho \nabla \cdot \mathbf{v}=-\left(\frac{\partial}{\partial \mathrm{t}}+\mathbf{v} \cdot \nabla\right) \rho
$$

In view of Eq. 2.13, it is readily apparent that the right side of Eq. 2.21 is a quantity of order $\rho^{\prime}$, which is small. One therefore usually uses the approximation

$$
\nabla \cdot \mathbf{v}=0
$$

in problems of thermal convection. At this time, however, it is not necessary to make this assumption. Instead we write, with Eq. 2.15,

$$
\rho \nabla \cdot \mathbf{v}=\rho_{\mathrm{m}}\left(\frac{\partial}{\partial \mathrm{t}}+\mathbf{v} \cdot \nabla\right)\left(\frac{\mathrm{T}^{\prime}}{\mathrm{T}_{\mathrm{m}}}+\frac{\Delta \mathrm{T}}{\mathrm{T}_{\mathrm{m}}}-\frac{\Delta \mathrm{p}}{\mathrm{p}_{\mathrm{m}}}\right)
$$

Notice that $\Delta \mathrm{p} / \mathrm{p}_{\mathrm{m}}$ depends on $\mathrm{z}$ only. We therefore find

$$
\left(\frac{\partial}{\partial \mathrm{t}}+\mathbf{v} \cdot \nabla\right) \frac{\Delta \mathrm{p}}{\mathrm{p}_{\mathrm{m}}}=\frac{\mathrm{w}}{\mathrm{p}_{\mathrm{m}}} \frac{\partial \mathrm{p}_{\mathrm{R}}}{\partial \mathrm{z}} \approx \frac{\mathrm{wg}}{\mathrm{p}_{\mathrm{m}}} \rho_{\mathrm{m}}
$$

where use has been made of Eq. 2.5, and a term multiplied with $\rho_{R}-\rho_{m}$ has been neglected. Consequently the continuity equation becomes, with the equation of state,

$$
\nabla \cdot \mathbf{v}=\frac{\mathrm{R} \rho_{\mathrm{m}}}{\mathrm{p}_{\mathrm{m}}}\left(\frac{\partial}{\partial \mathrm{t}}+\mathbf{v} \cdot \nabla\right) \mathrm{T}+\frac{\rho_{\mathrm{m}}}{\mathrm{p}_{\mathrm{m}}} \mathrm{wg}
$$

Elimination of the term $\mathrm{p} \nabla \cdot \mathbf{v} \sim \mathrm{p}_{\mathrm{m}} \nabla \cdot \mathrm{v}$ in the energy equation, Eq. 2.15 , by means of Eq. 2.25 yields to the same approximation:

$$
\rho_{\mathrm{m}} \mathrm{c}_{\mathrm{p}}\left(\frac{\partial}{\partial \mathrm{t}}+\mathbf{v} \cdot \nabla\right) \mathrm{T}=\mathrm{k} \nabla^{2} \mathrm{~T}^{\prime}-\mathrm{wg} \rho_{\mathrm{m}}+\rho_{\mathrm{m}} \phi+\rho_{\mathrm{m}} \mathrm{c}_{\mathrm{p}} R
$$

where the relation

$$
c_{p}=c_{v}+R
$$

has been used.

An alternative form of the energy equation is found by splitting $T$ into $T^{\prime}+T_{R}$, and, since the latter is a function of $\mathrm{z}$ only, we obtain, after division by $\rho_{\mathrm{m}} \mathrm{c}_{\mathrm{p}}$ and use of Eq. 2.11,

$$
\left(\frac{\partial}{\partial \mathrm{t}}+\mathrm{v} \cdot \nabla\right) \mathrm{T}^{\prime}=\frac{\mathrm{k}}{\rho_{\mathrm{m}} \mathrm{c}_{\mathrm{p}}} \nabla^{2} \mathrm{~T}^{\prime}+\frac{\phi}{\mathrm{c}_{\mathrm{p}}}+\mathbb{R}
$$


Finally, the equation of motion is given to

$$
\left(\frac{\partial}{\partial \mathrm{t}}+\mathbf{v} \cdot \nabla\right) \mathbf{v}=-\frac{1}{\rho_{\mathrm{m}}} \nabla \mathrm{p}+\mathrm{g} \frac{\mathrm{T}^{\prime}}{\mathrm{T}_{\mathrm{m}}} \mathbf{k}+\nu \nabla^{2} \mathbf{v}
$$

where it has been assumed that $\nabla^{2} \mathbf{v} \gg \nabla(\nabla \cdot \mathbf{v})$, which can be inferred either from the same order-of-magnitude arguments for $\nabla \cdot \mathrm{v}$ as led to Eq. 2.25 or from Eq. 2.22.

Equations 2.22 or $2.25,2.26$ or 2.28 , and 2.29 form the basic set of equations for calculating the thermally stratified planetary boundary layer to the Boussinesq approximation. It should be noted that they are essentially identical to the set of equations obtained by assuming the atmosphere to be incompressible, except that the mean temperature must be replaced by the mean potential temperature. Such simplifications, however, cannot be obtained for a moist atmosphere where in addition a conservation equation for water vapor appears which is coupled to the other equations through the equation of state.

Even for dry air it is readily apparent that the Boussinesq equations are not solvable in their general form because of their highly nonlinear character. Further simplifications arise in the important special case of free convection, for which the pertinent equations are given in Chap. 3 .

\section{TURBULENT MOTIONS IN STRATIFIED SHEAR FLOWS TO THE BOUSSINESO APPROXIMATION}

\section{The Time-Averaged Equations for Turbulent Motions in a Thin Fluid Layer}

We shall now give the equations equivalent to Eqs. 2.22, 2.28, and 2.29 for a turbulent flow field whose velocity and temperature fields are described by

$$
\mathbf{v}=\overline{\mathbf{v}}+\mathbf{v}^{\prime}
$$

where

$$
\begin{array}{r}
\mathbf{v} \cdot \mathbf{i}=\mathbf{u}=\bar{u}(z)+u^{\prime}(x, y, z, t) \\
\mathbf{v} \cdot \mathbf{j}=v=v^{\prime}(x, y, z, t) \\
\mathbf{v} \cdot \mathbf{k}=w=w^{\prime}(x, y, z, t)
\end{array}
$$

and

$$
\theta=\bar{\theta}(\mathrm{z})+\theta^{\prime}(\mathrm{x}, \mathrm{y}, \mathrm{z}, \mathrm{t})
$$


where the overbar denotes time averages, and $\mathrm{u}^{\prime}, \mathrm{v}^{\prime}$, and $\mathrm{w}^{\prime}$ are the fluctuatıng velocities of the flow field, which, to the approximation of $\mathrm{Eq} 222$, are related by the continuity equation,

$$
\nabla \quad \mathbf{v}^{\prime}=0
$$

The change from $T$ to $\theta$ reflects that deviations from the potential temperature are to be used

We shall now restate the equations of motion in terms of these quantities First, Eq 229 s readily seen to become the set of equations

$$
\begin{aligned}
& \frac{\partial\left(\overline{\mathrm{u}}+\mathrm{u}^{\prime}\right)}{\partial \mathrm{t}}+\left(\overline{\mathrm{u}}+\mathrm{u}^{\prime}\right) \frac{\partial \mathrm{u}^{\prime}}{\partial \mathrm{x}}+\mathrm{v}^{\prime} \frac{\partial \mathrm{u}^{\prime}}{\partial \mathrm{y}}+\mathrm{w}^{\prime} \frac{\partial\left(\overline{\mathrm{u}}+\mathrm{u}^{\prime}\right)}{\partial \mathrm{z}}=-\frac{1}{\rho} \frac{\partial\left(\mathrm{p}^{\prime}+\overline{\mathrm{p}}\right)}{\partial \mathrm{x}}+\nu \nabla^{2}\left(\mathrm{u}^{\prime}+\overline{\mathrm{u}}\right) \\
& \frac{\partial \mathrm{v}^{\prime}}{\partial \mathrm{t}}+\left(\overline{\mathrm{u}}+\mathrm{u}^{\prime}\right) \frac{\partial \mathrm{v}^{\prime}}{\partial \mathrm{x}}+\mathrm{v}^{\prime} \frac{\partial \mathrm{v}^{\prime}}{\partial \mathrm{y}}+\mathrm{w}^{\prime} \frac{\partial \mathrm{v}^{\prime}}{\partial \mathrm{z}}=-\frac{1}{\rho} \frac{\partial \mathrm{p}^{\prime}}{\partial \mathrm{y}}+\nu \nabla^{2} \mathrm{v}^{\prime} \\
& \frac{\partial \mathrm{w}^{\prime}}{\partial \mathrm{t}}+\left(\overline{\mathrm{u}}+\mathrm{u}^{\prime}\right) \frac{\partial \mathrm{w}^{\prime}}{\partial \mathrm{x}}+\mathrm{v}^{\prime} \frac{\partial \mathrm{w}^{\prime}}{\partial \mathrm{z}}+\mathrm{w}^{\prime} \frac{\partial \mathrm{w}^{\prime}}{\partial \mathrm{z}}=-\frac{1}{\rho} \frac{\partial \mathrm{p}^{\prime}}{\partial \mathrm{z}}+\nu \nabla^{2} \mathrm{w}^{\prime}-\mathrm{g} \frac{\rho^{\prime}}{\rho}
\end{aligned}
$$

where the subscript $\mathrm{m}$ has been dropped We note that these equations are identical to those in Chap 1, except for the last term in Eq 236 , which is the only term due to thermal stratification In addition, however, we have to consider the energy equation, which becomes, from $\mathrm{Eq} 228$,

$$
\frac{\partial\left(\bar{\theta}+\theta^{\prime}\right)}{\partial \mathrm{t}}+\left(\overline{\mathrm{u}}+\mathrm{u}^{\prime}\right) \frac{\partial \theta^{\prime}}{\partial \mathrm{x}}+\mathrm{v}^{\prime} \frac{\partial \theta^{\prime}}{\partial \mathrm{y}}+\mathrm{w}^{\prime} \frac{\partial\left(\bar{\theta}+\theta^{\prime}\right)}{\partial \mathrm{z}}=\overline{\mathrm{k}} \nabla^{2}\left(\bar{\theta}+\theta^{\prime}\right)+\frac{\phi}{\mathrm{c}_{\mathrm{p}}}+\mathbb{R}
$$

where $\overline{\mathrm{k}}=\mathrm{k} / \rho_{\mathrm{m}} \mathrm{c}_{\mathrm{p}}$ has been used It is advantageous to express the dissipation $\phi$ also by its mean and fluctuation parts Thereby, one must realize that in turbulent motion the dissipation consists of three parts (1) that due to the mean motion which is denoted by $\epsilon_{\mathrm{m}}$, (2) the mean value $\epsilon$ of the dissipation of the turbulent motion, and (3) the deviation $\epsilon^{\prime}$ of the instantaneous value from the mean, so that

$$
\phi=\epsilon_{\mathbf{m}}+\epsilon+\epsilon^{\prime}
$$

From these sets we furst obtain the time averages

$$
\frac{\partial \overline{\mathrm{u}}}{\partial \mathrm{t}}=-\frac{1}{\rho} \frac{\partial \overline{\mathrm{p}}}{\partial \mathrm{x}}+\frac{\partial}{\partial \mathrm{z}}\left(v \frac{\partial \overline{\mathrm{u}}}{\partial \mathrm{z}}-\overline{\mathrm{u}^{\prime} \mathrm{w}^{\prime}}\right)
$$

and

$$
\frac{\partial \bar{\theta}}{\partial \mathrm{t}}+\frac{\partial}{\partial \mathrm{z}} \overline{\mathrm{w}^{\prime} \theta^{\prime}}=\overline{\mathrm{k}} \nabla^{2} \bar{\theta}+\frac{\epsilon_{\mathrm{m}}+\epsilon}{\mathrm{c}_{\mathrm{p}}}
$$


where use has been made of plane homogeneity and of the continuty equation, Eq 2.33 .

If we multiply Eq. 2.34 by $\overrightarrow{\mathrm{u}}$ and average over time, we obtain the change of kunetic energy of the mean motion

$$
\frac{1}{2} \frac{\overrightarrow{\mathrm{u}}^{2}}{\mathrm{dt}}=-\frac{1}{\rho} \overline{\mathrm{u}} \frac{\partial \overline{\mathrm{p}}}{\partial \mathrm{x}}+\overline{\mathrm{u}} \frac{\partial}{\partial \mathrm{z}}\left(\nu \frac{\partial \overline{\mathrm{u}}}{\partial \mathrm{z}}-\overline{\mathrm{u}^{\prime} \mathrm{w}^{\prime}}\right)
$$

where the operator $(d / d t)=(\partial / \partial t)+\bar{v} \cdot \nabla$. Multiplyıng Eqs. 2.34 to 2.36 with $u^{\prime}, v^{\prime}$, and $w^{\prime}$, respectively, time averaging, and adding yield the energy equation of turbulent motion, as in Chap 1

$$
\overline{\frac{\mathrm{dq}}{\mathrm{dt}}}+\frac{\partial}{\partial \mathrm{z}} \overline{\mathrm{w}^{\prime}\left(\frac{\mathrm{p}^{\prime}}{\rho}+\mathrm{q}^{2}\right)}=-\overline{\mathrm{u}^{\prime} \mathrm{w}^{\prime}} \frac{\partial \overline{\mathrm{u}}}{\partial \mathrm{z}}+\frac{\mathrm{g}}{\mathrm{T}} \overline{\mathrm{w}^{\prime} \theta^{\prime}}-\epsilon
$$

in which only the term $(\mathrm{g} / \mathrm{T}) \overline{\mathrm{w}^{\prime} \theta^{\prime}}$ reflects the effect of buoyancy, it is the buoyancy-production term of turbulence.

If Eq. 2.37 is multiplied by $\theta^{\prime}$ and the time average is taken, a similar equation is obtained for the conservation of turbulent temperature fluctuations

$$
\begin{gathered}
\frac{1}{2} \frac{\mathrm{d} \theta^{\prime 2}}{\mathrm{dt}}=-\frac{1}{2} \overline{\mathrm{u}_{1}^{\prime} \frac{\partial \theta^{\prime 2}}{\partial \mathrm{x}_{1}}}-\overline{\mathrm{w}^{\prime} \theta^{\prime}} \frac{\partial \bar{\theta}}{\partial \mathrm{z}}+\overline{\overline{\mathrm{k}} \theta^{\prime} \nabla^{2} \theta^{\prime}}+\overline{\mathcal{R}^{\prime} \theta^{\prime}} \\
\text { I }
\end{gathered}
$$

in which the fluctuating part of the dissipation is neglected.* In Eq. 2.43, term I is the change in temperature fluctuations. With the help of the continuity equation, the advection term, term II, can be expressed as

$$
\overline{u_{1}^{\prime} \frac{\partial \theta^{\prime 2}}{\partial x_{1}}}=\frac{\partial}{\partial x_{1}} \overline{u_{1}^{\prime} \theta^{\prime 2}}=\frac{\partial}{\partial z} \overline{w^{\prime} \theta^{\prime 2}}
$$

as is usual. Term III denotes the production of fluctuating temperatures through the interaction of the turbulent heat flux with the temperature gradient Term IV combines the loss of energy from the fluctuatıng temperatures into mean temperatures (a loss that is usually denoted by $-\epsilon_{t}$ ) with the molecular-heat-diffusion term (a term that is small compared to term II and therefore negligible). Term $\mathrm{V}$ is the radiative

\footnotetext{
*In neglecting $\overline{\epsilon^{\prime} \theta^{\prime}}$, we are aware that the fluctuating dissipation is mostly associated with the high-frequency end of the velocity spectrum. Therefore, even if the dissipation were correlated strongly with the temperature, the covariances would be very small because $\theta^{\prime}$ is small in the frequencies where $\epsilon^{\prime}$ has significant magnitudes
} 
change of turbulent temperature fluctuations which will be discussed below. Then the energy equation becomes

$$
\frac{\overline{\mathrm{d}} \overline{\theta^{\prime 2}}}{\mathrm{dt}}=-\frac{\partial}{\partial \mathrm{z}} \overline{\mathrm{w}^{\prime} \theta^{\prime 2}}-2 \overline{\mathrm{w}^{\prime} \theta^{\prime}} \frac{\partial \overline{\mathrm{T}}}{\partial \mathrm{z}}-2 \epsilon_{\mathrm{t}}+2 \overline{\mathrm{R}^{\prime} \theta^{\prime}}
$$

Equations 2.42 and 2.45 together form the most useful set of conservation equations for stratified turbulent flow. Other conservation equations, for example for $\mathrm{w}^{\prime} \theta^{\prime}$, can be obtained from the set of Eqs. 2.34 to 2.37 by multiplication with a fluctuating component and averaging in a straightforward manner. For example, to the same approximation as Eq. 2.45, we find for $\mathrm{dw}^{\prime} \theta^{\prime} / \mathrm{dt}$ [but with the term $\left(\theta^{\prime} / \rho\right)\left(\partial \mathrm{p}^{\prime} / \partial \mathrm{z}\right)$ neglected] :

$$
\frac{\mathrm{dw}^{\prime} \theta^{\prime}}{\mathrm{dt}}+\frac{\partial \mathrm{w}^{\prime 2} \theta^{\prime}}{\partial \mathrm{z}}+\overline{\mathrm{w}^{\prime 2}} \frac{\partial \mathrm{T}}{\partial \mathrm{z}}+\frac{\overline{\theta^{\prime 2}}}{\mathrm{~T}} \mathrm{~g}=\epsilon_{\mathrm{w} \theta}+\overline{\mathrm{w}^{\prime} \mathrm{R}^{\prime}}
$$

where

$$
\epsilon_{\mathrm{w} \theta}=\overline{\mathrm{k}} \overline{\mathrm{w}^{\prime} \nabla^{2} \theta^{\prime}}+\overline{\nu \theta^{\prime} \nabla^{2} \mathrm{w}^{\prime}}
$$

is a dissipation term.

\section{The Effect of Humidity on the Conservation Equations}

The presence of water vapor in the air changes the conservation equations due to two important physical effects. First, since the specific weight of water vapor is larger than that of air, the density of moist air differs from that of dry air, thus changing the hydrostatic equilibrium. Humidity fluctuations contribute to density fluctuations, and the density effect of water vapor is therefore found both in the mean (reference) state and in the energy balance of the turbulent motion. The former can be accounted for by defining a virtual potential temperature as that equivalent dry-air potential temperature which would produce a mean reference state of stratification that equals the observed state of the moist air. The latter gives rise to a buoyancy flux because the fluctuations in the water-vapor concentration affect the buoyancy term in the energy equations of the turbulent motion.

The other physical process that is affected by the presence of water vapor is radiation. Dry air is transparent to radiation, but in moist air the long-wave radiation from the ground is partially absorbed by the vapor and is thus changed into sensible heat that will, of course, influence the temperature structure. The problem of radiation absorption by vapor is much more difficult to treat because of the highly nonlinear radiation equation. That problem will be outlined in the next subsection. Here, we shall consider the effect of water vapor on the density structure of the atmospheric boundary layer. 
The equation of state applies to any ideal gas, and for moist air it is as valid as for dry air. It is, however, convenient in atmospheric calculations to operate with the properties of dry air and, when the air contains water vapor, to express its effect in a virtual temperature $T_{v}$, which is derived as follows. Let the subscript A denote dry-air conditions and let the subscript $\mathrm{W}$ pertain to water vapor. Then the total density of a unit volume of moist air is

$$
\rho=\rho_{\mathrm{A}}+\rho_{\mathrm{W}}
$$

where each of the constituents corresponds to its own partial pressure. Since both vapor and air are approxımately ideal gases, it follows that

$$
\rho=\frac{\mathrm{p}-\mathrm{p}_{\mathrm{W}}}{\mathrm{R}_{\mathrm{A}} \mathrm{T}}+\frac{\mathrm{p}_{\mathrm{W}}}{\mathrm{R}_{\mathrm{W}} \mathrm{T}}
$$

where $p_{W}$ is the partial pressure of water vapor By introducing $a=R_{A} / R_{W}=0.622$, we can write Eq 2.49 as

$$
\rho=\frac{\mathrm{p}}{\mathrm{R}_{\mathrm{A}} \mathrm{T}}\left[1+\frac{\mathrm{p}_{\mathrm{W}}}{\mathrm{p}}(\mathrm{a}-1)\right]
$$

Now $\mathrm{p}_{\mathrm{W}} / \mathrm{p}$ is proportional to the absolute humidity $\xi$, 1.e , the weight of vapor per unit we1ght of moist air, as is seen from the relation

$$
\xi=\frac{\rho_{\mathrm{W}}}{\rho}=\frac{\mathrm{p}_{\mathrm{W}}}{\rho} \frac{\mathrm{R}}{\mathrm{R}_{\mathrm{W}}} \approx \mathrm{a} \frac{\mathrm{p}_{\mathrm{W}}}{\mathrm{p}}
$$

where it has been assumed that the amount of vapor is very small, so that $\mathrm{R} \approx \mathrm{R}_{\mathrm{A}}$. It follows that

$$
\rho=\frac{\mathrm{p}}{\mathrm{RT}}(1-06 \xi) \approx \frac{\mathrm{p}}{\mathrm{RT}(1+06 \xi)}=\frac{\mathrm{p}}{\mathrm{RT}_{\mathrm{v}}}
$$

where $T_{v}$ is the virtual temperature

$$
T_{v}=T(1+06 \xi)
$$

The use of the virtual temperature in heu of the actual temperature makes it possible to use the equation of state for moist air just like that for dry air However, it must be noted that the virtual temperature is not directly related to the heat content of a volume of air, and thus the conservation equations for heat do not apply directly. For this reason, only the case of dry air will be considered in the sections on "Mean Velocity Distribution in Stratıfied-Boundary-Layer Flows" and "Temperature Profiles and Heat Flux in Stratified Air" 
Further complications arise when the humidity content becomes large enough for condensation to take place or when liquid water is present which can evaporate We shall not discuss such cases here

Next we consider the contribution of humidity fluctuations to the balance of turbulent energy They will affect only the buoyancy term of the turbulent-energy Eq. 2 42. The total density fluctuations are expressed by means of Eqs 216 and 2.52 to

$$
\frac{\rho^{\prime}}{\rho}=-\left(\frac{\theta^{\prime}}{\mathrm{T}}+0.6 \xi^{\prime}\right)
$$

Introducing this expression into buoyancy term and averaging give Eq. 2.42 in the following form

$$
\left.\overline{\frac{\mathrm{dq}}{\mathrm{dt}}}+\frac{\partial}{\partial \mathrm{z}} \overline{\mathrm{w}^{\prime}\left(\frac{\mathrm{p}^{\prime}}{\rho}+\mathrm{q}^{2}\right)}=-\overline{\mathrm{u}^{\prime} \mathrm{w}^{\prime}} \frac{\partial \overline{\mathrm{u}}}{\partial \mathrm{z}}+\frac{\mathrm{g}}{\mathrm{T}} \overline{\left(\mathrm{w}^{\prime} \theta^{\prime}\right.}+0.6 \overline{\mathrm{w}^{\prime} \xi^{\prime} \mathrm{T}}\right)-\epsilon
$$

We may define the fluxes (per unit area) as

$$
\mathrm{H}=\rho \mathrm{c}_{\mathrm{p}} \overline{\mathrm{w}^{\prime} \theta^{\prime}}
$$

where $\mathrm{H}$ is the flux of sensible heat and

$$
\mathrm{E}=\rho \overline{\mathrm{w}^{\prime} \xi^{\prime}}
$$

which is the flux of water vapor. The latter is related to the quantity

$$
\mathrm{Q}=\rho \overline{\mathrm{Lw}^{\prime} \xi^{\prime}}=\mathrm{LE}
$$

where $Q$ is the latent heat flux. The coefficient $L$ is the latent heat of condensation, 1.e., that amount of heat which would be released if the water vapor present in a unit volume were condensated to water. With these expressions the buoyancy term becomes

$$
-\frac{\mathrm{g}}{\rho} \overline{\rho^{\prime} \mathrm{w}^{\prime}}=\frac{\mathrm{g}}{\rho \mathrm{T} \mathrm{c}_{\mathrm{p}}}\left(\mathrm{H}+0.6 \frac{\mathrm{c}_{\mathrm{p}} \mathrm{T}}{\mathrm{L}} \mathrm{Q}\right)
$$

When $\mathrm{T}$ is about $20^{\circ} \mathrm{C}$, then

$$
0.6 \frac{c_{p} T}{L} \approx 0.07
$$

so that $\mathrm{Q}$ must be about 15 times larger than $\mathrm{H}$ to be of equal importance (Ball, 1960). 
A measure of the effect of water vapor on the turbulent flux is the Bowen ratio defined to

$$
B=\frac{\mathrm{H}_{0}}{\mathrm{Q}_{0}}
$$

where the subscript zero pertains to conditions at ground level The Bowen ratio evidently depends on the amount of moisture that is avallable at the ground surface, ranging from infinity in the desert to values of about 01 over the open ocean. In the latter case the contribution of the latent heat to the buoyancy term cannot be neglected, and one must infer that for progressively moister surface conditions the latent heat increases in importance On the other hand, it is clear that for a surface in equilibrium with the incoming radiation the buoyancy term 1s, under otherwise identical conditions, substantially reduced by the process of evaporation This follows because in that case the heat $\Delta \mathrm{H}$ avalable for heating the arr at the surface, $1 \mathrm{e}$, the difference between net radiation and heat conduction into the ground, is balanced by the sensible heat flux $\mathrm{H}$ and the latent heat of evaporation $\mathrm{LE}, 1 \mathrm{e}$,

$$
\Delta \mathrm{H}=\mathrm{H}+\mathrm{LE}=\mathrm{H}\left(1+\frac{1}{\mathrm{~B}}\right)
$$

Over a dry surface LE is zero, and it follows from Eq 255 that the buoyancy term near the ground (but above the thin conduction layer directly at the ground) is equal to

$$
-\frac{\mathrm{g} \overline{\rho^{\prime} \mathrm{w}^{\prime}}}{\rho}=\frac{\mathrm{g} \Delta \mathrm{H}}{\rho \mathrm{T} c_{\mathrm{p}}}
$$

On the other hand, for a Bowen ratio of 01 , the flux of sensible heat $H$ is only $1 / 11$ $\Delta \mathrm{H}$, the rest of the heat is used to convert liquid water into vapor Therefore the buoyancy term has increased only to

$$
-\frac{\mathrm{g}}{\rho} \overline{\rho^{\prime} \mathrm{w}^{\prime}}=\frac{\mathrm{gH}}{\rho \mathrm{T} \mathrm{c}_{\mathrm{p}}}\left(1+\frac{007}{\mathrm{~B}}\right)=\frac{\mathrm{g}}{\rho \mathrm{T}} \frac{\Delta \mathrm{H}}{\mathrm{c}_{\mathrm{p}}} \frac{17}{11}
$$

I e , over a saturated surface the buoyancy is only about $15 \%$ of what it would be over the desert, at the same amount of $\Delta \mathbf{H}$

It is obvious that the result of Eq 264 cannot be applied directly to any particular surface at any particular time because too many factors have been disregarded which govern the energy-balance Eq 262 To compare the relative effectiveness of buoyancy over different surfaces requires that the local and temporal terms of the energy budget be considered Also, in a moist air the long-wave radiation of the ground is "trapped" by the water vapor in the air, whereas dry air is transparent to it Therefore in a moist atmosphere it is necessary to assess the heat flux due to radiation 


\section{The Energy Equation with Radiation Heat Transfer}

Since the sun's short-wave radiation reaches the earth's surface with very little attenuation by the air in the atmospheric boundary layer and since the long-wave radiation from the ground can be trapped by the lower atmosphere because of the absorption characteristics of moist atmospheric air, it follows that some of the changes of the heat content of the atmosphere must be associated with radiation from the ground Here we shall not give the derivation of the radiation equation for mean radiation effects on the temperature distribution, for which reference may be made to Fleagle and Businger (1963) or the more fundamental and elaborate treatment by Goody (1964). It is usually assumed that the direct effect of radiation on the mean temperature distribution is small, at least in air with low humidity, although, in a paper by Gayevskaya (1962) in which extensive reference is made to the meteorolog1cal literature on the subject, the conclusion was reached that at least in stable nighttıme conditions radiation may be a significant factor in determining the mean temperature distribution near the ground (Elliott, 1964). Coantic and Sequin (1970) have calculated that over water at moderate winds radiation may account for as much as $40 \%$ of the total heat flux and for a highly nonlinear distribution of the heat flux with height. The absolute value of the heat flux is always increased by radiation, and it is, strictly speaking, not permissible to neglect radiation over water Over land, radiative heat fluxes are smaller, and in our discussions we chose to ignore the effect of radiation on mean temperatures, not so much because it is negligible but because its effect adds further complications to the already difficult discussion of thermally stratified boundary layers. We must, however, keep in mind that, at least very near the ground, radiation can be an important factor which at least rules out the possibility of determining experimentally the heat flux at the ground by assuming a state of pure conduction and measuring the temperature gradient very near the ground.

Radiation also affects the stability of the atmosphere in the layer close to the ground, as was shown by Townsend (1958b). A fluid particle whose instantaneous temperature deviates from the local mean temperature by an amount $\theta^{\prime}$ radiates to its surroundings or receives radiation from its surroundings, and, since the largest effect of radiation is felt at the shortest distance from the particle, the radiation has the tendency to strongly smooth out the fluctuations over short distances, 1.e., the effect of radiation is felt more strongly in the high-frequency end of the temperature-fluctuation spectrum. The overall effect of radiation is a reduction in the mean square value of the turbulent temperature fluctuation. Formally the balance equation for turbulent temperature fluctuations (Eq. 2.45) becomes

$$
\frac{\mathrm{d} \overline{\theta^{\prime 2}}}{\mathrm{dt}}=-\frac{\partial}{\partial \mathrm{z}} \overline{\mathrm{w}^{\prime} \theta^{\prime 2}}+2 \overline{\mathrm{w}^{\prime} \theta^{\prime}} \frac{\partial \overline{\mathrm{T}}}{\partial \mathrm{z}}-2 \epsilon_{\mathrm{t}}-2 \beta \overline{\theta^{\prime 2}}
$$

where the coefficient $\beta$ is defined in terms of the fluctuatıng radiative heat flux $R^{\prime}$ by

$$
\beta=-\frac{\overline{\mathrm{R}^{\prime} \theta^{\prime}}}{\overline{\theta^{\prime 2}}}
$$


The negative sign in Eq. 2.66 reflects the fact that turbulent temperature fluctuations are always destroyed by radiation.

Note that the radiation flux does not appear directly in the energy-balance

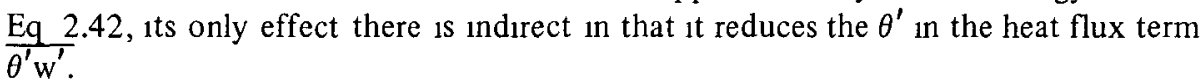

It is evident that $\mathrm{Eq} 266$ is not of much use unless some relation can be found between $\mathrm{R}^{\prime}$ and $\theta^{\prime}$ which defines $\beta$ Such a relation has been developed by Townsend (1958b), to whose paper reference is made for detalls It is found that, when the temperature fluctuation is small compared to the mean temperature $\overline{\mathrm{T}}$, the radiation equation can be linearized (see also Spiegel, 1957) and the coefficient $\beta(\mathrm{k})$ associated with the disturbance wave number $k$ becomes (as given by Goody, 1964)

$$
\beta(\mathrm{k})=\frac{4 \pi}{\rho c_{\mathrm{p}}} \frac{\overline{\mathrm{d}} \mathrm{d}}{\mathrm{dT}} \tilde{\mathrm{k}}(\mathrm{k})
$$

where $\mathrm{B}$ is the Planck function and $\overline{\mathrm{dB} / \mathrm{dT}}$ is the integral of the derivative of the Planck function over all wavelengths of the radiation, equal to $56 \times 10^{3} \mathrm{ergs} /\left(\mathrm{cm}^{2}\right)(\mathrm{sec})$ (deg). The quantity $\widetilde{\mathrm{k}}$ is the effective absorption coefficient For high wave numbers $(\mathrm{k} \rightarrow \infty)$, the absorption coefficient is independent of wave numbers and given to

$$
\tilde{\mathrm{k}}=203 \rho_{\mathrm{w}}+96 \rho_{\mathrm{c}}+136 \rho_{\mathrm{o}}\left(\mathrm{cm}^{-1}\right)
$$

where the subscripts on the density $\rho$ stand for water, carbon dioxide, and ozone, respectively The numerical constants pertain to a temperature of $290^{\circ} \mathrm{K}$ For other temperatures the corresponding $\beta$ at high wave numbers can be found by supposing $\beta(\mathrm{k})$ to vary as the cube of the absolute temperature

At medium frequencies the absorption coefficient becomes a function of frequency. Since by far the largest contribution to the absorption coefficient comes from water vapor, it is sufficient to approximate it by that of water vapor. Results given by Goody (1964) are shown in Fig 2.1, where both the absorption coefficient and the wave number have been divided by the vapor density $\rho_{\mathrm{w}}$. With this curve the total radiation loss can be found by integration over the spectrum of $\beta(\mathrm{k}) \mathrm{S}_{\theta}(\mathrm{k})$, where $\mathrm{S}_{\theta}$ is the wave-number spectrum of the temperature-fluctuation variance Unless exact results are required, it is usually sufficient to estimate radiation losses by multiplying $\overline{\theta^{\prime 2}}$ by a suitable average value of $\beta$ found with the help of $F_{1 g} 2.1$

\section{The Effect of Buoyancy on Turbulence and the Richardson Number}

The presence of a heat flux significantly changes the energy balance, but not in such a way that the overall turbulence is reduced uniformly. Instead, buoyancy acts selectively and directly only on the vertical component of turbulence, whereas production by shear stresses mainly adds to the longitudinal, or $u^{\prime}$, component of the turbulence. This is readily seen from the conservation equations for turbulent energy, 


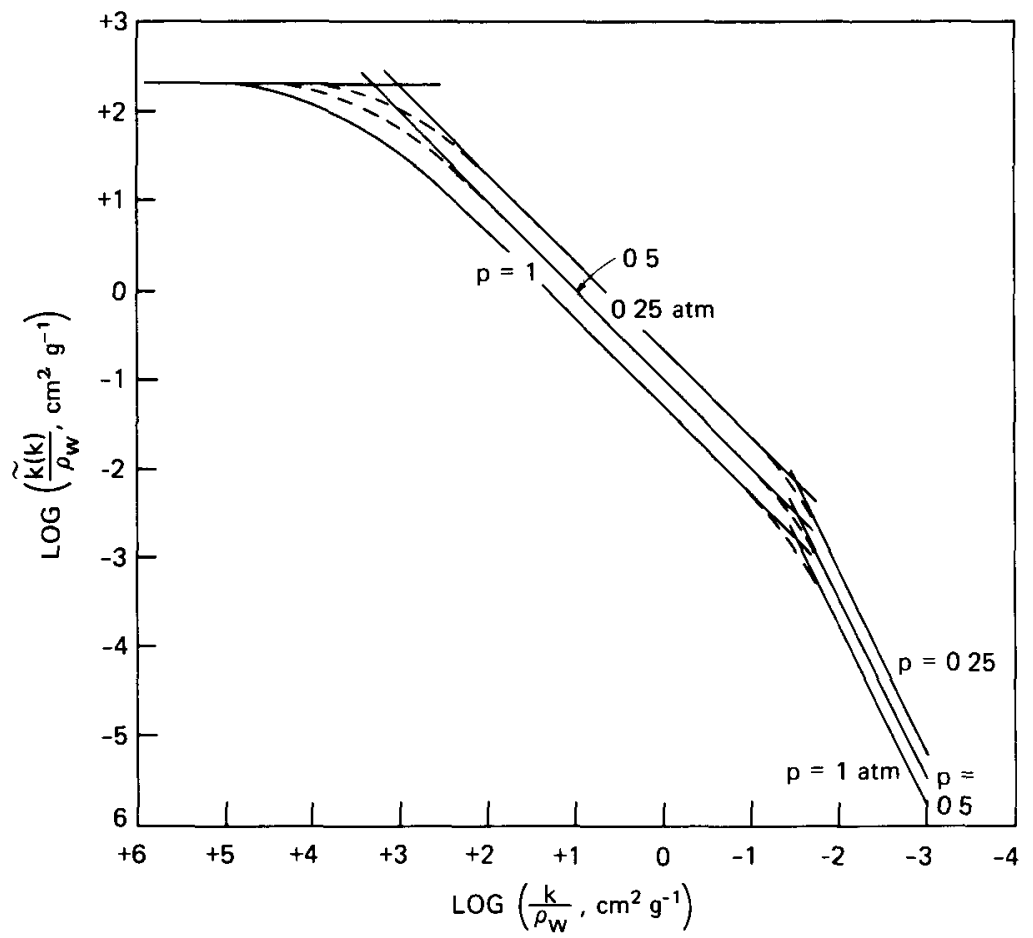

Fig. 2.1 Effective absorption coefficient for water vapor in air, at different atmospheric pressures. (From Goody, 1964.)

which for each of the three components separately reduces, for the case of a unform layer of fluid, to

$$
\begin{aligned}
& \frac{1}{2} \frac{\partial \overline{u^{\prime 2}}}{\partial t}+\frac{\partial}{\partial z}\left[\frac{1}{2} \overline{u^{\prime 2} w^{\prime}}-\frac{1}{2} \nu \frac{\partial \overline{u^{\prime 2}}}{\partial z}\right]=\frac{1}{\rho} \overline{p^{\prime} \frac{\partial u^{\prime}}{\partial x}}-\overline{u^{\prime} w^{\prime}} \frac{\partial \bar{u}}{\partial z}-\epsilon_{u} \\
& \frac{1}{2} \frac{\partial \overline{v^{\prime 2}}}{\partial \mathrm{t}}+\frac{\partial}{\partial \mathrm{z}}\left[\frac{1}{2} \overline{\mathrm{v}^{\prime 2} \mathrm{w}^{\prime}}-\frac{1}{2} \nu \frac{\partial \overline{\mathrm{v}^{\prime 2}}}{\partial \mathrm{z}}\right]=\frac{1}{\rho} \overline{\mathrm{p}^{\prime}} \overline{\frac{\partial \mathrm{v}^{\prime}}{\partial \mathrm{v}}}-\epsilon_{\mathrm{v}} \\
& \frac{1}{2} \frac{\partial \overline{\mathrm{w}^{\prime 2}}}{\partial \mathrm{t}}+\frac{\partial}{\partial \mathrm{z}}\left[\frac{1}{2} \overline{\mathrm{w}^{\prime 3}}-\frac{1}{2} \nu \frac{\partial \overline{\mathrm{w}^{\prime 2}}}{\partial \mathrm{z}}\right]=\frac{1}{\rho} \overline{\mathrm{p}^{\prime}} \frac{\partial \mathrm{w}^{\prime}}{\partial \mathrm{z}}+\frac{\mathrm{g}}{\mathrm{T}} \overline{\mathrm{w}^{\prime} \theta^{\prime}}-\epsilon_{\mathrm{w}}
\end{aligned}
$$

These equations are obtained in the same manner as Eq. 242 When there is no shear, any anisotropy in the turbulence enters into the vertical component from the buoyancy term, and the other two components obtain energy only indirectly through the redistributing action of the diffusion terms (in the square brackets) and the pressure term. These terms have the tendency to homogenize the turbulence and to make the turbulence field lose connection with its generation history. 
In the case of shear flows considered here, the longitudinal component acts as a source term of energy for the lateral and vertical turbulence which locally raises the turbulence level of that particular component until the energy flux into it is balanced by the dissipation term. In the absence of buoyancy, there is not much preference between the $\mathrm{v}^{\prime}$ and $\mathrm{w}^{\prime}$ components; they tend to be very similar in magnitude and distribution. In the absence of shear, the energy goes from the buoyancy term into the vertical turbulence component, which becomes the source of energy for the other two components. Finally, when both buoyancy and shear are acting, the turbulence becomes even less homogeneous. In particular, when the flow is stable (downward heat flux), energy is fed into the $u^{\prime}$ component by the shear work, is redistributed over all components by the diffusion and pressure terms, and is dissipated by the combined actions of dissipation and buoyancy with the result that the vertical component contains the least energy of all three components. A local measure of the anisotropy is the ratio of the two production terms

$$
R_{\mathrm{f}}=\frac{\frac{\mathrm{g}}{\mathrm{T}} \overline{\mathrm{w}^{\prime} \theta^{\prime}}}{+\overline{\mathrm{u}^{\prime} \mathrm{w}^{\prime}} \frac{\partial \overline{\mathrm{u}}}{\partial \mathrm{z}}}
$$

which is the flux Richardson number $\left(R_{f}\right)$. Its dynamical significance lies in the fact that it gives a measure of the relative importance of the buoyancy term as compared to the shear-production term. Large flux Richardson numbers signify that buoyancy dominates the turbulence structure, whereas at Richardson numbers near zero, buoyancy is of little effect on the flow.

The flux Richardson number is a function of the distance from the ground and thus a local quantity. To see this, consider the (near-neutral) case where the velocity distribution is logarithmic, and heat flux and shear stress are independent of height and equal to $\mathrm{H}_{0}$ and $\rho \mathrm{u}_{*}^{2}$, respectively. Then

$$
\frac{\partial \overline{\mathrm{u}}}{\partial \mathrm{z}}=\frac{\mathrm{u}_{*}}{\kappa \mathrm{z}}
$$

where $k$ is von Karman's constant, and the flux Richardson number becomes

$$
\mathrm{R}_{\mathrm{f}}=\frac{\frac{\mathrm{g}}{\mathrm{T}} \mathrm{H}_{0}}{-\frac{\mathrm{u}_{*}^{3}}{\kappa \mathrm{z}}}=\frac{\mathrm{z}}{\mathrm{L}}
$$

where the important quantity $L$ is a length incorporating the effect of buoyancy:

$$
\mathrm{L}=-\frac{\mathrm{u}_{*}^{3}}{\kappa \frac{\mathrm{g}}{\mathrm{T}} \mathrm{H}_{0}}
$$


It is readily seen that $L$ is equal to the distance from the ground at which the two production terms are equal.

Note that the equality of $z / L$ and $R_{f}$ holds only when the velocity profile is logarithmic, i.e., when the velocity profile is not affected by buoyancy, except perhaps for a possible influence on the roughness height $\mathrm{z}_{0}$. For different profile shapes or when heat flux and/or shear are functions of distance, the flux Richardson number is of more complicated functional form, but it is reasonable that this function should depend on $\mathrm{z} / \mathrm{L}$ only as in the near-neutral case. This is in fact so. The relation between flux Richardson number and $\mathrm{z} / \mathrm{L}$ for stable flows is well documented for wind-tunnel boundary layers along a smooth surface by data of Arya and Plate (1969), at least for small $\mathrm{z} / \mathrm{L}$, and for unstable conditions by field data such as the ones obtained at the Kansas Field Station of the Air Force Cambridge Research Laboratories and reported by Businger et al. (1970).

Measurements of flux Richardson numbers are not easily made because they require parallel measurements of both heat and momentum flux. More readily obtained is a related quantity called the gradient Richardson number (Ri), defined to:

$$
R i=\frac{\frac{g}{T} \frac{d T}{d z}}{\left(\frac{d \bar{u}}{d z}\right)^{2}}
$$

When the fluxes and gradients are connected by means of the eddy-diffusivity assumptions

$$
\begin{aligned}
& \overline{-u^{\prime} w^{\prime}}=K_{M} \frac{\partial \bar{u}}{\partial z} \\
& \overline{w^{\prime} \theta^{\prime}}=-K_{H} \frac{\partial T}{\partial z}
\end{aligned}
$$

where $\mathrm{K}_{\mathrm{M}}$ and $\mathrm{K}_{\mathrm{H}}$ are the exchange coefficients (or eddy diffusivities) for momentum and heat, respectively, then the flux Richardson number is related to Ri by

$$
R_{f}=\frac{K_{H}}{K_{M}} R i
$$

Both Richardson numbers have different dynamical significance. The flux Richardson number is a quantity describing the effect of local stability on the turbulence field. A very small value of $R_{\mathbf{f}}$ indicates that the flow is near neutral, and its velocity distribution is described by the logarithmic profile. This condition can arise either because $L$ is a large quantity, which implies that $H_{0}$ is small or $u_{*}$ large, or because $\mathrm{z}$ is small. Thus, even in a strongly stratified flow, there always exists in a shear flow a 
layer near the ground in which the flow is characteristic of neutrally stratified flow, i.e., it can be described above any viscous sublayer that may exist by the logarithmic law. Consequently, for small values of z/L, Eq. 2.74 must hold and is thus seen to be the asymptotic form for the flux Richardson number for small values of $z / L$.

When the flow is unstable, the flux Richardson number is negative. With increasing instability (or at larger distances from the ground), the shear effect on the flow becomes less and less important until at some flux Richardson number the presence of shear no longer affects the dynamics of the flow field significantly: the flow has become driven entirely by buoyancy and is called free-convection flow. Some controversy has resulted about whether there exists an abrupt transition, at some critical value of $R_{f}$ or $z / L$, from forced- to free-convection regimes. Priestley (1955) in a pioneering study of this matter has inferred, from a change in the mean-temperature profiles, that at $-z / L \geqslant 0.03$ or thereabouts free convection sets in (see also the discussion in Priestley, 1959), but it is currently no longer held that the transition from forced to free convection in the atmosphere is abrupt. We shall defer a discussion of the flow during free convection to the next chapter.

For stable flows, corresponding to positive Richardson numbers, buoyancy tends to reduce the turbulence level. Again, for small positive values of $z / L$, the flow remains near neutral, but with increase in $\mathrm{z} / \mathrm{L}$ the turbulent intensities become smaller, and it is likely that there exists a critical value of $R_{f}$ beyond which turbulence can no longer exist. This problem has been attacked theoretically from the direction of instability of stably stratified flow with a velocity gradient, the simplest case being one in which the density distribution is described by

$$
\frac{\mathrm{d} \rho}{\rho \mathrm{dz}}=-\mathrm{b}
$$

and the velocity is linear and equal to

$$
\frac{\mathrm{du}}{\mathrm{dz}}=\mathrm{a}
$$

Taylor (1931) found for this case that, if the fluid is bounded from below by a horizontal plane and extends to infinity above, it is stable at all disturbances of small amplitude so long as $\mathrm{a}^{2}<4 \mathrm{gb}$, or so long as

$$
\mathrm{Ri}=\frac{\frac{\mathrm{g}}{\rho} \frac{\mathrm{d} \rho}{\mathrm{dz}}}{\left(\frac{\mathrm{du}}{\mathrm{dz}}\right)^{2}}>\frac{1}{4}
$$

a result that was found valid by many other investigators (see Thorpe, 1969) also for more general profiles.

The gradient Richardson number is thus seen to determine the stability of a stratified fluid without turbulence to small perturbations, i.e., it is a measure of the 
onset of turbulence In Taylor's model the effect of viscosity on the stability was neglected, as well as the effect of velocity-profile curvature An investigation of the stability of a laminar boundary layer against small disturbances was made by Schlichting (1935) who described the velocity distribution in terms of the Blasius laminar-boundary-layer profile with a constant density gradient $\mathrm{He}$ found that all small perturbations were damped for

$$
\mathrm{R}_{\mathrm{w}} \geqslant 00417
$$

where $R_{w_{w}}$ is the gradient Richardson number based on the velocity gradient near the wall

The instability problem of a stably stratified fluid is not necessarily related to the problem of suppression of existing turbulence, turbulence has been observed to exist at practically all gradient Richardson numbers (but see the discussion below) The parameter determining the persistence of existing turbulence appears to be the flux Richardson number Attempts have been made by Townsend (1958) and Ellison (1957) to obtain a numerical value for the critical flux Richardson number from the conservation equations for turbulent motion, Eqs 242, 245, and 246 When the motion is steady and uniform, when the air is dry, and when gradients of triple correlations can be neglected, these equations reduce to

$$
\begin{gathered}
\epsilon_{\mathrm{t}}=-\overline{\mathrm{w}^{\prime} \theta^{\prime}} \frac{\mathrm{d} \overline{\mathrm{T}}}{\mathrm{dz}} \\
\epsilon=-\overline{\mathrm{u}^{\prime} \mathrm{w}^{\prime}} \frac{\mathrm{d} \overline{\mathrm{u}}}{\mathrm{dz}}+\frac{\mathrm{g}}{\mathrm{T}} \overline{\mathrm{w}^{\prime} \theta^{\prime}}
\end{gathered}
$$

and

$$
\epsilon_{\mathrm{w} \theta}=\overline{\mathrm{w}^{\prime 2}} \frac{\mathrm{d} \overline{\mathrm{T}}}{\mathrm{dz}}+\frac{\mathrm{g}}{\mathrm{T}} \overline{\theta^{\prime 2}}
$$

A series of assumptions is needed to close the set of equations, both to relate fluxes to gradients and to relate the dissipation terms to other turbulent quantities A simple assumption is to relate dissipations to the conserved quantities of the form

$$
\begin{gathered}
\epsilon=\frac{\overline{q^{2}}}{\tau_{1}} \\
\epsilon_{\mathrm{t}}=\frac{\overline{\theta^{\prime 2}}}{2 \tau_{2}}
\end{gathered}
$$

and 


$$
\epsilon_{\mathrm{w} \theta}=\frac{\overline{\mathrm{w}^{\prime} \theta^{\prime}}}{\tau_{3}}
$$

where $\tau_{1}, \tau_{2}$, and $\tau_{3}$ are characteristic times of the motion, which in general are functions of space and of the structure of turbulence.

Further results require assumptions relating the fluxes to the parameters of the mean velocity and temperature field. For a constant stress layer,

$$
\overline{-\mathrm{u}^{\prime} \mathrm{w}^{\prime}}=\mathrm{u}_{*}^{2}
$$

and

$$
\overline{-\mathrm{w}^{\prime} \theta^{\prime}}=\mathrm{H}_{0}
$$

are approximately valid. With Eqs. 277 and 278 relating fluxes to gradients, Ellison (1957) found for this situation that the ratio $K_{H} / K_{M}$ is expressed in terms of the flux Richardson number $R_{f}$

$$
\frac{\mathrm{K}_{\mathrm{H}}}{\mathrm{K}_{\mathrm{M}}}=\frac{\overline{\mathrm{q}^{2}} \overline{\mathrm{w}^{\prime 2}}}{\mathrm{u}_{*}^{4} \frac{\tau_{1}}{\tau_{3}}\left(1-\mathrm{R}_{\mathrm{f}}\right)^{2}}\left[1-\mathrm{R}_{\mathrm{f}}\left(1+\frac{\tau_{2}}{\tau_{1}} \frac{\overline{\mathrm{q}^{2}}}{\overline{\mathrm{w}^{\prime 2}}}\right)\right]
$$

Flux Richardson number and gradient Richardson numbers are related by Eq 2.79, consequently, if $R_{f}$ approaches a critical value and $R_{1}$ can be any value, it has been argued by Ellison (1957) that $\mathrm{K}_{\mathrm{H}} / \mathrm{K}_{\mathrm{M}}$ must approach zero as $\mathrm{R}_{\mathrm{f}}$ approaches $\mathrm{K}_{\mathrm{f}}$ critical If this is so, then one possibility in obtaining a critical flux Richardson number is to set the square bracket of Eq 292 equal to zero. Suitable assumptions on the ratio $\left(\tau_{2} / \tau_{1}\right)\left(\mathrm{q}^{2} / \mathrm{w}^{\prime 2}\right)$ permit a critical $R_{f}$ value to be found Note that at $R_{f}=0$, $\mathrm{K}_{\mathrm{H}} / \mathrm{K}_{\mathrm{M}}$ has the value

$$
\mathrm{b}=\left.\frac{\mathrm{K}_{\mathrm{H}}}{\mathrm{K}_{\mathrm{M}}}\right|_{0}=\frac{\overline{\mathrm{q}^{2}} \overline{\mathrm{w}^{\prime n}}}{\mathrm{u}_{*}^{4}} \frac{\tau_{3}}{\tau_{1}}
$$

and, if this ratio is independent of stability, we may write Eq. 2.87 in the form

$$
\frac{K_{H}}{K_{M}}=b \frac{1-\frac{R_{f}}{R_{f} \text { crit }}}{\left(1-R_{f}\right)^{2}}
$$

which, if plotted as a family of curves of $K_{H} / K_{M}$ vs $R_{l}$ with $R_{f \text { crit }}$ as third parameters, yields curves as shown in Fig. 22 Ellison and Turner (1960) made some experiments on stratified pipe flow, and their results together with those of Proudman (1953) are indicated in Fig. 2.2. The stratification was obtained by means of salt in the 




Fig. 2.2 Comparison of experimental results of Ellison and Turner (1960) with the theory of Ellison (1957). The values of $R_{f}$ crit, are marked on the theoretical curves. The experiments of Ellison and Turner (1960) pertain to salt solutions in the laboratory. The data reported by Proudman (1953) were taken in the Kattegat.

water, so that the exchange coefficient is that for salt diffusion denoted by $\mathrm{K}_{\mathrm{S}}$ instead of the heat diffusivity $\mathrm{K}_{\mathrm{H}}$. The accuracy of the results is farly low Ellison and Turner (1960) point out that the error due to inaccurate determinations of the velocity gradient for their data can amount to as much as $20 \%$, thus leading to possible errors of $40 \%$ in the gradient Richardson number. However, the data seem to indicate that the critical flux Richardson number is likely to be smaller than 02 and not much different from the value of 0.17 inferred by Ellison (1957) in his earlier paper

An interesting conclusion from the experiments of Ellison and Turner is that $b$ has a constant numerical value of about 1.4, very similar to the value found in fully forced convection near the center line of a pipe, or to the atmospheric value of 12 reported by Record and Cramer (1966). Recently Businger et al. (1970) have reported a value of 1.35 .

If both $b$ and $R_{f}$ crit are constants, then $K_{H} / K_{M}$ as well as $R_{1}$ should be functions of flux Richardson number only, as follows from Eq. 2.94, or because $R_{\mathrm{f}}$ is a function of $z / L$ they should depend on $z / L$ only. Empirical results of this dependency have been collected from many sources and are reproduced in Figs. 2.3 and 24 . For $\mathrm{R}_{1}$ the $\mathrm{z} / \mathrm{L}$ dependency is well confirmed, and excellent agreement is obtained between field and laboratory. For $\mathrm{K}_{\mathrm{H}} / \mathrm{K}_{\mathrm{M}}$ the scatter of the data is very large, and a closer inspection of results such as those of Arya and Plate (1969) indicates that the ratio does not only depend on $\mathrm{z} / \mathrm{L}$ but also decreases slightly directly with height This is really no surprise since $\mathrm{K}_{\mathrm{H}} / \mathrm{K}_{\mathrm{M}}$ relates dynamically quite different quantities Slight variations of $b$ with distance from the solid boundary are known to exist in pipe flow 




Fig. 2.3 The relation between $\mathrm{z} / \mathrm{L}$ and $\mathrm{R}_{1}$ for the whole range of stability Field data from Businger et al. (1970), laboratory data in stable region from Arya and Plate (1969).

(Schlichtıng, 1968) where $b$ decreases from a value of about 14 near the center to 1 at the wall For turbulent boundary layers the dependency of $b$ on $z$ is not yet established, and the published results appear to be conflictıng (Kestın and Richardson, 1963) For near-neutral unstably stratıfıed boundary layers, Johnson (1959) reports a value which is about 1 at the wall and which increases to about 2 at the edge of the boundary layer, whereas the results of Arya and Plate (1969) for a stably stratified boundary layer appear to indicate a value of $b$ that decreases with $\mathrm{z}$ Apart from the possible variation of $b$ with distance from the ground, it is also found that the value of $R_{f \text { crit. }}$ which is obtained from Ellison's model is not constant. To obtain a value of $R_{f}$ crit. $=0.17$, Ellison assumed $\tau_{2} / \tau_{1}=1$ and $\overline{q^{2}} / \overline{w^{\prime 2}}=5.5$. The latter number corresponds approximately to conditions near neutral, but it is in general not constant in boundary layers except perhaps at some distance from the ground Recent experimental data of Arya (1968) in the constant-stress layer along a smooth boundary have falled to confirm Ellison's assumptions. Some results from Arya's study are reproduced in Table 2.1. The ratio $\overline{\mathrm{q}^{2}} / \sqrt{\mathrm{w}^{\prime 2}}$ is not far from being constant, although it varies slightly both with $\mathrm{z}$ and $\mathrm{L}$, but the ratio of the time scales depends strongly on both height and stability The reason for this difference is at present unexplained 


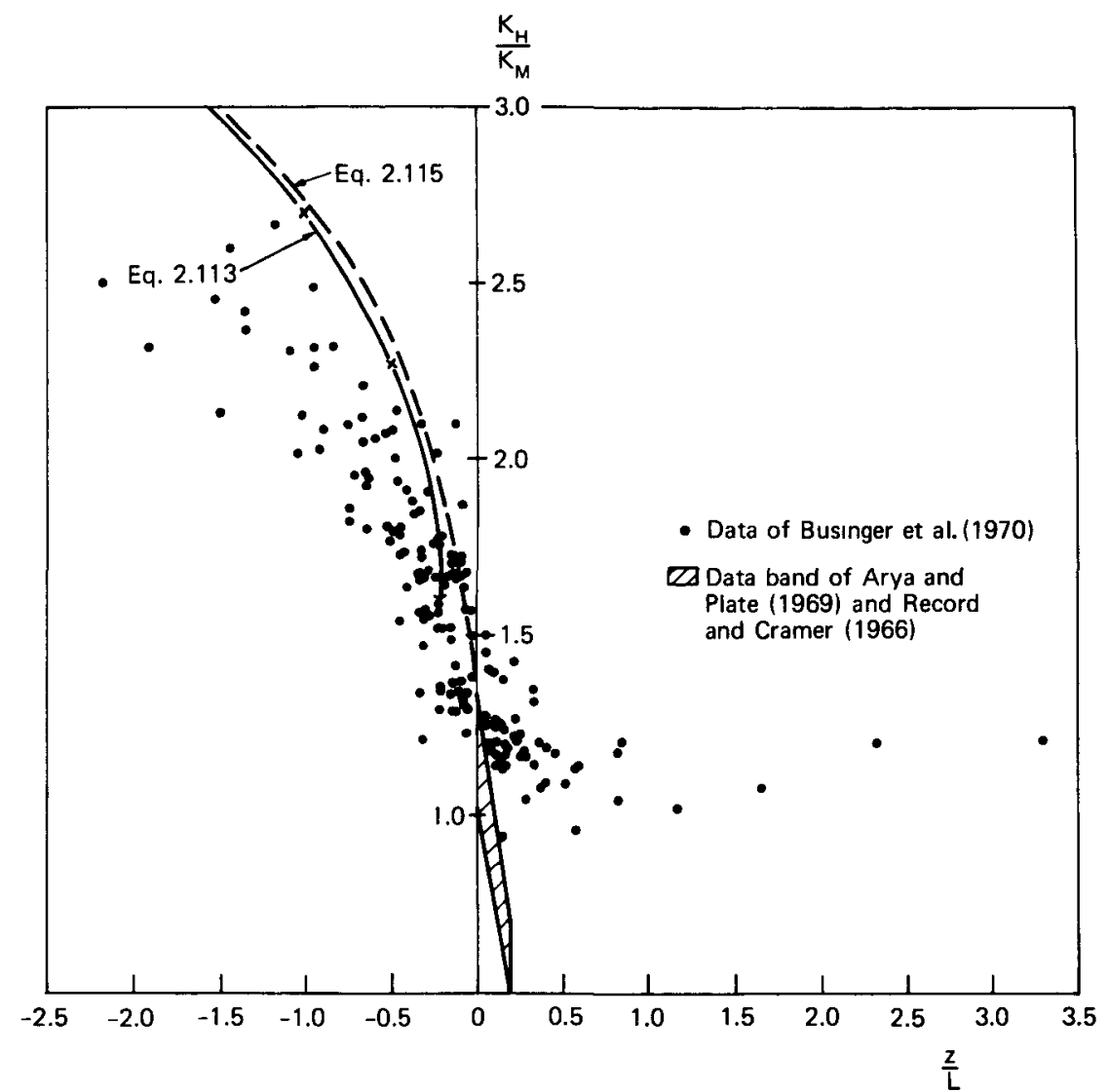

Fig. 2.4 The relation between $\mathrm{K}_{\mathrm{H}} / \mathrm{K}_{\mathrm{M}}$ and $\mathrm{z} / \mathrm{L}$. Field data from Bussinger et al. (1970) and Record and Cramer (1966); laboratory data in stable region from Arya and Plate (1969). The dashed curve corresponds to Eq. 2.115 and the solid curve to Eq. 2.113 .

Table 2,1

PARAMETERS OF ELLISON'S THEORY DETERMINED FROM LABORATORY DATA OF ARYA (1968)

\begin{tabular}{lllcc}
\hline \multicolumn{1}{c}{$\mathbf{z} / \mathbf{L}$} & \multicolumn{1}{c}{$\mathbf{R}_{\mathbf{f}}$} & $\tau_{2} / \tau_{1}$ & $\overline{q^{2}} / 2 \mathrm{w}^{\prime 2}$ & $\mathbf{q}^{2} \tau_{2} / 2 \mathrm{w}^{2} \tau_{1}$ \\
\hline 0.0035 & 0.0033 & 0.34 & 5.72 & 1.96 \\
0.0087 & 0.0075 & 0.43 & 6.01 & 2.57 \\
0.043 & 0.021 & 0.77 & 6.47 & 4.97 \\
0.0063 & 0.006 & 0.28 & 5.16 & 1.43 \\
0.0155 & 0.011 & 0.38 & 5.14 & 1.95 \\
0.079 & 0.033 & 0.67 & 5.93 & 3.99 \\
0.0084 & 0.0078 & 0.25 & 4.86 & 1.23 \\
0.0212 & 0.013 & 0.38 & 4.88 & 1.86 \\
0.105 & 0.039 & 0.68 & 5.77 & 3.94 \\
\hline
\end{tabular}


Recently, in his studies of the stability of thin turbulent layers in the Mediterranean, Wood (1969) found that there seems to be a critical gradient Richardson number equal to 1 at which turbulence breaks down. His survey of the experimental evidence indicated that there was no case at which a gradient Richardson number of 1 was exceeded by turbulent flow. His conclusion was that the argument reproduced above is not valid and that instead of a flux Richardson number of $0.17 \mathrm{a}$ better criterion for the breakdown of turbulence is a gradient Richardson number of 1 . Consequently, if the gradient Richardson number lies between 0.25 and 1.0 , the state of the fluid depends on its past history: laminar flow remaining laminar until Ri drops below 0.25, and turbulent flow remaining turbulent until Ri increases above 1 . It should be noted that only the Kattegat data in Fig. 2.3 have Ri numbers larger than 1 whereas all other data are not inconsistent with Wood's view. Wood appears to have found that the method of obtaining the Ri number from the Kattegat data used by Ellison and Turner (1960) is not correct; revised values of Ri fall below 1.

At present, the experimental evidence of turbulence breakdown is not sufficient to decide in favor of either Ellison's or Wood's view, but there is no doubt that a Richardson-number hysteresis exists. It is the mechanism through which turbulent interfaces in stratified flow are kept well defined. If the interface is turbulent, its thickness increases and the gradients decrease leading to increases in $\mathrm{Ri}$ and $\mathrm{R}_{\mathrm{f}}$. Once the critical condition for turbulence breakdown is exceeded, the flow becomes laminar with accordingly much larger gradients that reduce $\mathrm{Ri}$. If the reduction is strong enough to lead to $\mathrm{Ri}<0.25$, then turbulence starts again and the cycle is repeated. This is the mechanism by which the stable layers above (and in evening and nighttime conditions below) the convection layer are separated from the turbulence field in the convection layer.

Ellison's model applies only to the region of constant stress and heat flux. Far away from the ground, it is no longer possible to assume these quantities as constant. Townsend (1958a) has made an analysis similar to that of Ellison for such layers. He obtained an expression for the flux Richardson number from Eqs. 2.87 and 2.88 by assuming that the shear stress and heat flux could be expressed in terms of the intensities $\sqrt{\overline{\mathrm{w}^{\prime 2}}}$ and $\sqrt{\overline{\theta^{\prime 2}}}$ through the coefficients $\mathrm{k}_{\mathrm{w}}$ and $\mathrm{k}_{\theta}$ :

$$
\overline{\mathrm{w}^{\prime} \theta^{\prime}}=\mathrm{k}_{\theta} \sqrt{\overline{\theta^{\prime 2}}} \sqrt{\overline{\mathrm{w}^{\prime 2}}}
$$

and

$$
\overline{u^{\prime} w^{\prime}}=k_{w} \overline{w^{\prime 2}}
$$

With the additional assumption of isotropy,

$$
\mathrm{q}^{2}=\frac{3}{2} \overline{\mathrm{w}^{\prime 2}}
$$


Townsend obtained for the flux Richardson number the expression

$$
\mathrm{R}_{\mathrm{f}}=\frac{1}{2}\left[1-\left(1-4 \frac{\tau_{2}}{\tau_{1}} \frac{\mathrm{k}_{\theta}^{2}}{\mathrm{k}_{\mathrm{w}}^{2}} \mathrm{R}_{1}\right)^{1 / 2}\right]
$$

from which he concluded that the maximum possible value of $R_{f} 1 s \frac{1}{2}$, corresponding to a critical gradient Richardson number of

$$
\mathrm{R}_{1}=\frac{1}{4} \frac{\tau_{1}}{\tau_{2}} \frac{\mathrm{k}_{\mathrm{w}}^{2}}{\mathrm{k}_{\theta}^{\frac{j}{2}}}
$$

Even if the ratios $\tau_{1} / \tau_{2}$ and $\mathrm{k}_{\mathrm{w}}^{2} / \mathrm{k}_{\theta}^{2}$ are independent of stability, they can depend on the space coordinates and thus a critical value of $\mathrm{R}_{1}$ is not likely to be uniquely defined The matter must be resolved by recourse to experiment The only existing data have been obtained in an experimental study of Webster (1964) in which turbulence was measured in a wind tunnel in a flow field that had both linear temperature and velocity profiles Owing to considerable difficulties in measurements of turbulence with a hot-wire anemometer in the presence of temperature fluctuations, the accuracy of Webster's data is not very high, leading, for example, to a neutral $\mathrm{K}_{\mathrm{H}} / \mathrm{K}_{\mathrm{M}}$ value of 22 , almost twice as high as observed by other techniques The data do seem to indicate, however, that the ratio $\tau_{1} / \tau_{2}$ in this flow is roughly constant and that there appears to be a well-defined critical flux Richardson number (which he found to be 035 , but again it is believed that this value might not be accurate)

It is of interest to consider the effect of humidity and radiation on the critical flux Richardson number Since water vapor in the aur affects only the heat flux, the magnitude of a critical Richardson number defined in terms of mass flux is likely to remain unchanged, whereas a flux Ruchardson number based on heat flux has a smaller critical value when the moisture flux is upward and a larger critical value when the moisture flux is downward Radiation, on the other hand, reduces temperature fluctuations in both stable and unstable flow The result is that, instead of the ratio $\tau_{1} / \tau_{2}$ in Eqs 292 and 298 , there appears the quantity $\tau_{1} / \tau_{2}+2 \beta \tau_{1}$, where $\beta$ has the meanıng assigned to it in Eq 266 Consequently the critical flux Richardson number is always increased by radiation (Townsend, 1958b, Goody, 1964) Note that the quantity $\beta \tau_{1}$ denotes the ratio of the decay time of the velocity fluctuation due to dissipation divided by the decay time of the temperature fluctuations due to radiation The effect of radiation on the flux Richardson number is therefore largest in a layer in which the turbulence is slowly decaying, 1e, it is associated mostly with low frequencies, while the temperature fluctuations are rapidly decaying, $1 \mathrm{e}$, they are associated with high frequencies

The discussion above shows that the process described in the introduction for the breakdown of the stable velocity profile near the ground proceeds as follows When the local flux Richardson number reaches a value near 02 at a particular critical height near the top of the inversion, turbulence locally starts to disappear, uncoupling the layers aloft and below the critical height The flow below the critical height is once 
more capable of becoming turbulent when the flow in the lower layer has accelerated so much that the gradient Richardson decreases below the value of about 025 Such a drop is usually caused by a reversal of the directions of the heat flux, but in some cases it might also occur even under inversion conditions as a result of acceleration through the geostrophic pressure field

\section{MEAN VELOCITY DISTRIBUTIONS IN STRATIFIED-BOUNDARY-LAYER FLOWS}

The set of Eqs 284 to 286 for the turbulent flow in a stratified boundary layer is not complete unless mean turbulent quantities can be expressed as functions of mean velocity and temperature distributions Such relations cannot be obtained from first principles, and the set of equations must be closed by empirically established, physically plausible relations Intuition breaks down very rapidly, however, with the complexity of the set of equations, and it is therefore customary to treat only the very simplest case of an atmospheric boundary layer which st1ll retains suff 1 cient features of the real situation This is the case of uniform flow in a constant-thickness layer parallel to a uniformly heated, uniformly rough ground at zero pressure gradient In that case Eq 244 becomes

$$
\frac{\mathrm{d}}{\mathrm{dz}}\left(\nu \frac{\mathrm{du}}{\mathrm{dz}}-\overline{\mathrm{u}^{\prime} \mathrm{w}^{\prime}}\right)=0
$$

or

$$
\mathrm{u}_{*}^{2}=\nu \frac{\mathrm{d} \overline{\mathrm{u}}}{\mathrm{dz}}-\overline{\mathrm{u}^{\prime} \mathrm{w}^{\prime}}
$$

where $u_{*}$ is the shear velocity The thermodynamic-energy Eq 240 becomes

$$
\frac{\mathrm{d}}{\mathrm{dz}}\left(\overline{\mathrm{w}^{\prime} \theta^{\prime}}-\overline{\mathrm{k}} \frac{\mathrm{d} \overline{\mathrm{T}}}{\mathrm{dz}}\right)=\frac{\epsilon}{\mathrm{c}_{\mathrm{p}}}
$$

where the dissipation of energy by the mean motion is neglected The term in parentheses is the heat flux $\mathrm{H}$ In turbulent flows the second term in parentheses is small and can be neglected, and in that case we obtain for the conservation of the kınetic and potential energy of the fluid the relation from Eq 245

$$
\mathrm{u}_{*}^{2} \frac{\mathrm{d} \overline{\mathrm{u}}}{\mathrm{dz}}+\frac{\mathrm{g}}{\mathrm{T}} \mathrm{H}=\epsilon
$$


If, because of the large value of $c_{p}$, the dissipation in Eq. 2.102 can be neglected, then it follows that the heat flux

$$
H=\overline{w^{\prime} \theta^{\prime}}-\bar{k} \frac{d \overline{\mathrm{T}}}{\mathrm{dz}}
$$

is independent of height. As was pointed out in Chap. 1, the shear stress is not exactly constant with height, and the same is true for the heat flux. To show this we assume that the velocity distribution is logarithmic in the fully turbulent region, i.e., $(\mathrm{du} / \mathrm{dz})=$ $\left(u_{*} / \kappa z\right)$. With this profile a first-order ordinary differential equation for the heat flux is obtained by eliminating $\epsilon$ between Eqs. 2.103 and 2.102, and the solution is

$$
\mathrm{H}=\mathrm{H}_{0}+\frac{\mathrm{u}_{*}^{3}}{\kappa \mathrm{c}_{\mathrm{p}}} \ln \frac{\mathrm{z}}{\mathrm{z}_{0}}
$$

where the terms at the right are the leading terms in a series expansion about $\mathrm{H}=\mathrm{H}_{0}$. The logarithmic term is usually small, typically, if $\mathrm{H}_{0}=20 \mathrm{~mW} / \mathrm{cm}^{2}, \mathrm{z}_{0}=1 \mathrm{~cm}$, and $c_{p}=10^{3} \mathrm{~m}^{2} /\left(\sec ^{2}\right)\left({ }^{\circ} \mathrm{K}\right)$; then, for a strong wind with $\mathrm{u}_{*}=1 \mathrm{~m} / \mathrm{sec}$, one finds that at about $\mathrm{z}=10 \mathrm{~m}$ the added term is $1.8 \mathrm{~mW} / \mathrm{cm}^{2}$ or about $10 \%$ of $\mathrm{H}_{0}$. Consequently an error resulting from neglecting the nonconstant term of the heat flux is, at most, of the same percentage as the error made in neglecting the effect of the Coriolis force and geostrophic pressure gradient on the shear stress. Usually it will be smaller because of smaller $u_{*}$ values and because it is compensated by radiation (Elliott, 1964).

With constant and presumably given values of $\mathrm{u}_{*}$ and $\mathrm{H}_{0}$, profiles of mean temperature and velocity can be calculated from Eqs. 2.84 and 2.86 provided that the relation between fluxes and gradients is known. Formally the relations can be expressed by Eqs. 2.77 and 2.78 through $\mathrm{K}_{\mathrm{H}}$ and $\mathrm{K}_{\mathrm{M}}$, i.e., the diffusivities of heat and momentum, respectively. These relations become meaningful only if it is possible to assign functional forms to $\mathrm{K}_{\mathrm{H}}$ and $\mathrm{K}_{\mathrm{M}}$ which make it possible to solve the defining equations. The purpose of this section is to present relations, based on empirical observations, which yield equations for velocity distributions in a thermally stratified flow.

\section{Velocity Distributions in the Stratified Layer Near the Ground}

In the heat-transfer literature, velocity profiles near a boundary are usually thought to be independent of the heat transfer from the boundary, i.e., it is assumed that the heat flux from the boundary does not affect the structure of the turbulence nearby. Most velocity-distribution laws used by heat-transfer engineers are identical to those discussed in Chap. 1, and calculations based on such profiles agree well with observations under widely varying conditions encountered in engineering applications.

For large heat flux and small velocity gradients, the velocity profiles of the stratified-boundary-layer flows begin to deviate from the logarithmic law near the boundary, and the change in profiles usually cannot be neglected in meteorology. 
Inasmuch as the Richardson number is the only new variable that enters the energy balance, it is logical to assume that it, or the quantity $z / L$, embodies the effect of stratification This concept is at the base of the similarity theory of Monin and Obukhov (1954) [see also Calder (1966)] who argued that gradients of velocity (and temperature) should be given by the nondimensional form

$$
\frac{\kappa \mathrm{z}}{\mathrm{u}_{*}} \frac{\mathrm{d} \overline{\mathrm{u}}}{\mathrm{dz}}=\phi_{\mathrm{M}}\left(\frac{\mathrm{z}}{\mathrm{L}}\right)
$$

For small values of $\mathrm{z} / \mathrm{L}$, it is possible to use the fact that the profile parameter $\phi_{\mathrm{M}}$ must become 1 as $L \rightarrow \infty$, and to develop the function $\phi_{M}$ in a Taylor series about the value 1 If the series is truncated after the furst term, the function becomes

$$
\phi_{\mathrm{M}}=1+\alpha \frac{\mathrm{z}}{\mathrm{L}}
$$

where $\alpha$ is an empirical constant This equation has been derived by many different authors in many different ways, for example, on the basis of mixing-length arguments by Kao (1959) and Businger (1955)

The formulation $\mathrm{Eq} 2107$ has been found appealing because the gradient, in contrast to the velocity distribution, is independent of the characteristic length of the surface, which is the roughness height $z_{0}$ This should, however, not be construed as implying that the profile is independent of the ground roughness The roughness effect enters indirectly through $\mathrm{u}_{*}$, which is a function of geostrophic conditions and of the stability of the flow The ground shear stress depends on the ground Rossby number, as discussed in Chap 1, and to this there must come an additional parameter or parameters depending on stability According to Batchelor (1953) the stability effect should be represented by a single parameter In a wind tunnel this stability parameter can be defined in terms of the characteristics of the layer by forming a characteristic Froude number of the friction layer (Batchelor, 1953) For the lower layer of the atmosphere, a characteristic length dimension is the roughness length $z_{0}$ One therefore would expect that for uniform terrain the geostrophic drag coefficient depends not only on the Rossby number but also on Businger's (1955) stability index $\mathrm{z}_{0} / \mathrm{L}$, which reflects the stability of the constant-stress layer, or

$$
\frac{\mathrm{u}_{*}}{\mathrm{G}}=\mathrm{f}\left(\mathrm{Ro}, \frac{\mathrm{z}_{0}}{\mathrm{~L}}\right)
$$

Plots for this functional dependency are difficult to obtain, mainly because of the complicated structure of the stratified boundary layer and its time-dependent nature, and at present $u_{*}$ is usually assumed to be a given quantity 


\section{Velocity Distributions in Unstable Stratification}

Integration of $\mathrm{Eq} 2107$ leads to a velocity profile which is logarithmic as in neutrally stratified flow but which has a correction term depending linearly on $z / L$ This so-called logarıthmic linear law has been introduced by many different workers (Ogura, 1952, Monın and Obukhov, 1954, Businger, 1955) with as many different coefficients $\alpha$ It is very likely that the best fitting value of $\alpha$ depends on the range of z/L values considered For unstable boundary layers, values ranging from 06 (Monin and Obukhov, 1954) to 6 (Taylor, 1960) have been reported (see also Zllitinkevich and Chalikov, 1968) As Taylor (1960) has pointed out, the small value of Monin and Obukhov is probably not correct because it was obtained for a range of $\mathrm{z} / \mathrm{L}$ values going much below that of -03 , at which approximately the Priestley free-convection regime begins The value $\alpha=6$ given by Taylor corresponds to $\mathrm{z} / \mathrm{L}$ between zero and -003 and is based on data of Swinbank (1955) and Rider (1954) In a later paper, Swinbank (1964) concluded on the basis of new experiments done over more-uniform terrain and from a new theoretical model for the wind profile that $\alpha$ should be less than 1 However, his theoretical model is based on a coordinate-stretching procedure of questionable validity, and the exponential wind profile which he derived is at most valid for unstable profiles (Barad, 1963, Plate and Lin, 1965) and is not widely accepted

The data of Swinbank (1964) are widely used by other authors, although there have been some questions on the accuracy of the shear-velocity values reported by Swinbank because they were calculated with a drag coefficient (based on the velocity at $z=1 \mathrm{~m}$ ) that was assumed constant independent of stability Pandolfo (1966) reanalyzed Swinbank's data and concluded that they correspond to a logarithmic linear law with a value of $\alpha$ of about 3 over the range $0>z / L>-003$, whereas Webb (1970) obtained a value of about 4

When the logarithmic linear law is inserted into Eq 272 , it follows for a constant-flux layer that the flux Richardson number is equal to

$$
\mathrm{R}_{\mathrm{f}}=\frac{\mathrm{z} / \mathrm{L}}{1+\alpha \frac{\mathrm{z}}{\mathrm{L}}}
$$

which can be used to infer the coefficient of $\alpha$ directly from measurements of fluxes But it is found, as in the case of profiles, that although the log linear relation can be made to $f_{1} t$ the observations well over selected ranges, it is not in very satisfactory agreement with the general trend of the data This is evident in field data of $\phi_{\mathrm{M}}$ such as shown in Fig 25 which were obtained by Businger et al (1970) By a suitable choice of the empirical constant $\alpha$, Eq 2107 can be made to fit the data at any desired point, but the trend of the curve is not in the direction of supporting a constant value of $\alpha$ Empirical improvements of the logarithmic linear law can be obtained in a number of ways, for example, by retaining higher order terms of the 


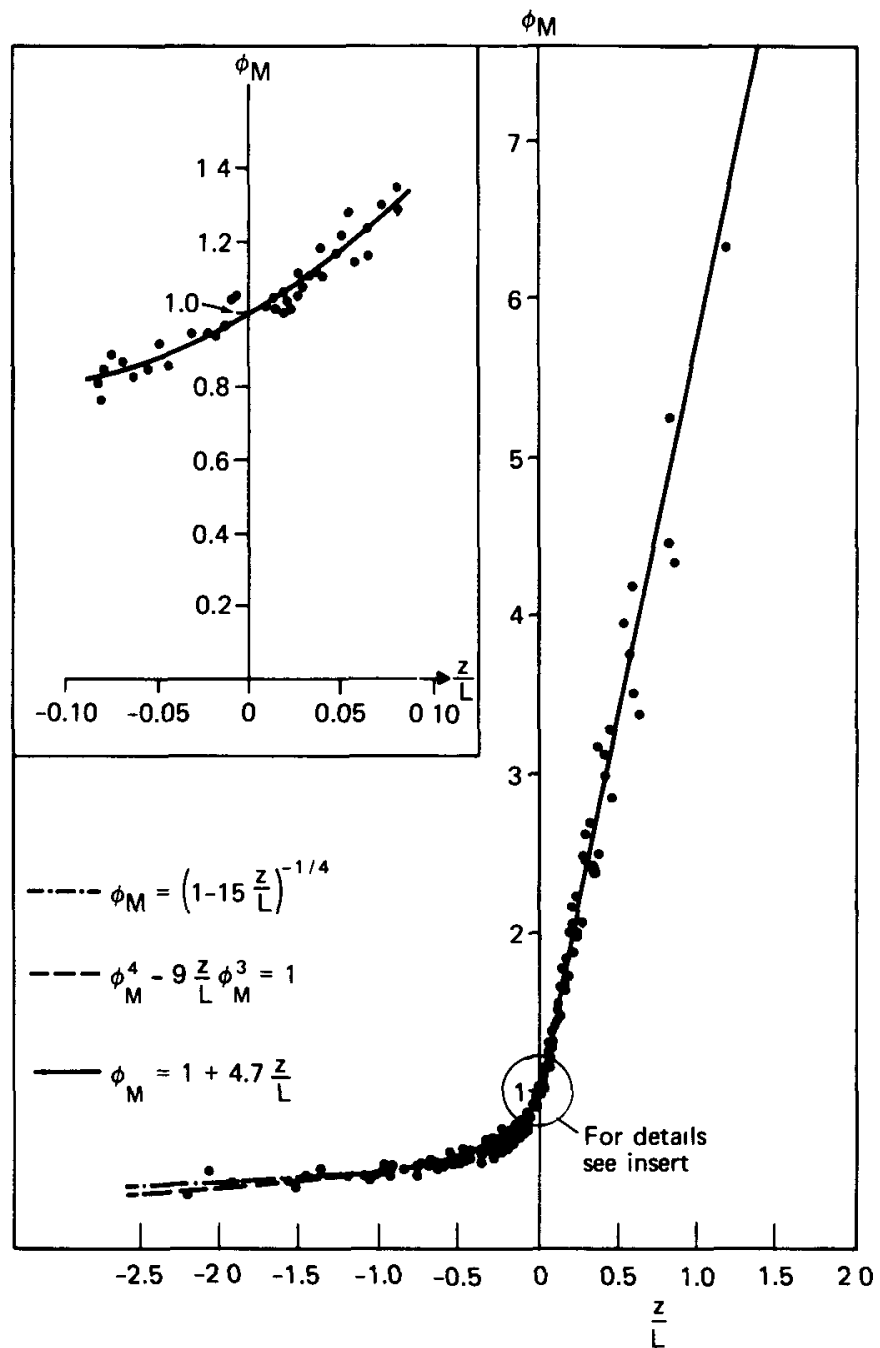

Fig. 2.5 Nondimensional wind shear as a function of $z / L$ Field data from Businger et al (1970), calculated with $\kappa=035$

Taylor series or by an empirical curve showing $\alpha$ vs. z/L. Most successful is probably the representation of the velocity profile in the form

$$
\phi_{\mathrm{M}}=\left(1-\alpha_{1} \mathrm{Ri}\right)^{\mathrm{n}}
$$

which was first suggested by Holzman (1943) with $n=-0.5$. Later authors have modified Holzman's equation and have concluded that it or a similarly constructed 
relation is applicable at least for unstable stratification. Typical is the formulation of Businger (1969) who found that

$$
\phi_{M}=\left(1-\beta \frac{Z}{L}\right)^{-025}
$$

where $\beta$ is about 15

With $\phi_{\mathrm{M}}$ given by an equation of the form $\mathrm{Eq} 2110$ or 2.111 and with the assumption $\mathrm{K}_{\mathrm{H}} / \mathrm{K}_{\mathrm{M}}=$ constant, it is possible to obtain the energy equation in the form

$$
\phi_{\mathrm{M}}^{4}-\gamma \frac{\mathrm{z}}{\mathrm{L}} \phi_{\mathrm{M}}^{3}=1
$$

where $\gamma$ is a constant that has to be found from experimental data This equation is called the "KEYPS" equation, which can be derived in a number of different ways. For large values of $z /-L$ and with a constant ratio $K_{H} / K_{M}$, it satisfies the $-4 / 3$ power law of Priestley (to be described in the section on "Temperature Profiles in Unstable Shear Flow") for the temperature distribution in the lower part of the free-convection layer. The equation (which is named after the initials of the writers who independently proposed it, among them Ellison, 1957, Yamamoto, 1959, and Panofsky, 1961) was initially only intended (Ellison, 1957) to be an interpolation formula between the profile near the critical Richardson number at stable conditions and the free-convection regime of Priestley (1955) The equation has, however, become well established as an empirical formula describing the unstable regime (Lumley and Panofsky, 1964), for which the equation agrees about as well with experimental data as any other proposed formula, as shown by Klug (1967) or by Panofsky in his discussion of the paper by Swinbank (1964), provided that $\gamma$ is chosen appropriately. Pandolfo (1966) uses a value of $\gamma=7$, whereas a value of 14 is quoted by Lumley and Panofsky (1964) Businger et al (1970) find $\gamma=9$ to yield good agreement of their data and Eq 2112 , as indicated in Fig 25

One of the shortcomings of the KEYPS equation is that it implies a constant ratio of $\mathrm{K}_{\mathrm{H}} / \mathrm{K}_{\mathrm{M}}$ in the free-convection range. Experimental evidence, in particular from Australian measurements of Swinbank (1964), does not support this assumption. Instead, Swinbank (1968) has shown that the data are well represented, above $\mathrm{z} /-\mathrm{L}=003$, by a power relation

$$
\frac{\mathrm{K}_{\mathrm{H}}}{\mathrm{K}_{\mathrm{M}}}=2.7\left(\frac{\mathrm{z}}{-\mathrm{L}}\right)^{025} \text { for } 0.03<\frac{\mathrm{z}}{-\mathrm{L}}<5
$$

Th1s equation was obtained directly from mean-profile data with the additional assumption of constant fluxes. In a parallel study of the same data, Pandolfo (1966) found that

$$
\frac{\mathrm{K}_{\mathrm{H}}}{\mathrm{K}_{\mathrm{M}}}=2\left(\frac{\mathrm{z}}{-\mathrm{L}}\right)^{0.167} \quad \text { for } \frac{\mathrm{z}}{-\mathrm{L}}>0.04
$$


Power-law equations do not, however, lead to a finite value of $K_{H} / K_{M}$ at $z /-L=0$, as they should They can, therefore, be valid only over certain ranges of $z / L$ values

Notice that Eq 2113 is the asymptotic form, for large $\mathrm{z} / \mathrm{L}$, of an interpolation equation

$$
\frac{\mathrm{K}_{\mathrm{H}}}{\mathrm{K}_{\mathrm{M}}}=\mathrm{b}\left(1-\beta \frac{\mathrm{z}}{\mathrm{L}}\right)^{025}
$$

which for $z / L=0$ reduces to the ratio $K_{H} / K_{M}$ at neutral stability $b$ If we use the value of 135 for $b$, it follows from Eq 2113 that the coefficient $\beta$ must be equal to about 16 Equation 2115 is in essential agreement with a formula proposed by Businger (1969) In the notation used here, $\beta$ appears in both Eqs 2111 and 2115 , by which the empirical observation is expressed that the two coefficients are about equal A comparison of the curves (Eqs 2113 and 2115 ) with the data of Businger et al (1970) yields unsatisfactory agreement between Swinbank's and Businger's data in the unstable region as is seen in Fig 24 Also, there is some discrepancy between the data of Arya and Plate (1969) and Record and Cramer (1966) on the one hand in the stable region, and those of Businger et al (1970) Further experiments must show where the error lies

\section{Velocity Distribution in Stable Stratifications}

For stable stratified-boundary-layer flow, the value of $\alpha$ in the logarithmic linear-law Eq 2107 that is most frequently quoted is 7 (McVehil, 1964), whereas Webb (1970), on the basis of Australian data and Businger et al (1970) as seen in Fig 24 , obtained a value of about 5 valid up to $z / L=1$ Velocity profiles are not very sensitive to the value of $\alpha$, and a more reliable test of the validity of the log linear law is obtained by plotting $\phi_{\mathrm{M}}$ vs $\mathrm{z} / \mathrm{L}$, according to Eq 2106 Such a plot of the wind-tunnel data reported by Arya and Plate (1969) is shown in Fig 26 , which is in substantial agreement with other laboratory data, such as those reported by Chuang and Cermak (1967) and Plate and Lin (1966) There seems to be reason to believe that the velocity distribution is logarithmic, $1 \mathrm{e}$, essentially like in neutral stratification up to $z / L=001$ For larger values of $z / L$, velocity distributions in stable flow are more suitably described by a power law of the form

$$
\phi_{M}=42\left(\frac{z}{L}\right)^{03}
$$

valid for $001 \leqslant z / L \leqslant 02$ This is a result similar to the earlier suggestion of Deacon (1949) who expressed the velocity profile by a power law with an exponent that depended on the stability Note that Arya and Plate's power law is just another way of expressing $\phi_{M}$ as a function of $z / L$ over some range of $z / L$ and thus reconcles Deacon's power-law approach with the similarity theory of Monin and Obukhov 




Fig. 2.6 Dimensionless wind shear as a function of $z / L$ in stably stratified flow Laboratory data from Arya and Plate (1969)

In stable stratifications, $\mathrm{K}_{\mathrm{H}} / \mathrm{K}_{\mathrm{M}}$ is also not constant, but it depends less strongly on $\mathrm{z} / \mathrm{L}$ as in the unstable case Arya and Plate found an exponent in the power-law relation between $\mathrm{K}_{\mathrm{H}} / \mathrm{K}_{\mathrm{M}}$ and $\mathrm{z} / \mathrm{L}$ more nearly equal to 01 than 025 , but, since the range of $z / L$ values covered by their experiments were small, this result is not conclusive For this reason they did not attempt to express Eq $2116 \mathrm{~m}$ a form similar to $\mathrm{Eq} 2111$, which would extrapolate to one at $\mathrm{z} / \mathrm{L} \rightarrow 0$

\section{TEMPERATURE PROFILES AND HEAT FLUX STRATIFIED AIR}

In fully forced convection, temperature profiles are found to also obey the logarithmic law This has been established mainly from laboratory experiments A most complete set of temperature profiles (for flow in smooth and rough pipes) has been reported by Gowen and Smith (1968) from which it follows that the temperature profile has an equation

$$
\frac{\theta_{\mathrm{w}}-\theta}{\mathrm{T}_{*}}=\frac{\kappa}{\kappa_{\mathrm{h}}} \ln \frac{\mathrm{z}}{\mathrm{z}_{\mathrm{Oh}}}
$$

where $T_{*}$ is called the shear temperature and $\kappa_{h}$ is the Karman constant for temperature The latter depends in general on the Prandtl number, but at the Prandtl number of air $(07)$ it is equal to the value 04 found for velocity distributions in neutral stratification The length $z_{0 h}$ corresponds to the roughness length of the 
velocity distributions but is not equal to it Since $z_{0}$ represents the dynamic action of the surface configuration on the velocity distribution and thus reflects separation and skin friction, the $z_{0 h}$ value should indicate the heat-transfer characteristics of the surface On smooth surfaces skin friction is the only type of friction that occurs, and for laminar flow Reynolds analogy holds according to which velocity and temperature distributions are identical in nondimensional form Although in turbulent flow it has not been established from first principles that Reynolds analogy is valid, it is usually assumed to hold for smooth surfaces (see Hinze, 1959, for a discussion of the validity of this assumption) Along rough surfaces the analogy almost certainly breaks down because, unlke momentum, heat transfer is not affected by pressures, and thus the heat transfer from the roughness elements takes place by conduction through the thin layer of fluid directly at the surfaces of contact between fluid and wall and depends very much on the heat conduction from the ground to the roughness elements, or on the direct heat that is radiated to the roughness element The matter is even more involved if the roughness consists of a vegetative cover where heat content and heat release are largely affected by evapotranspiration Near rough surfaces it is therefore necessary to exclude a thin layer in which no useful correlations between temperature and velocity can be found and to consider only the region which starts above the roughness and in which Reynolds analogy can be assumed A consequence of this is that the heat flux from the wall cannot be related to the aerodynamic properties in the simple manner obtained from Reynolds analogy, and separate investigations must be made for each roughness type because each of them will show a different relation between heat flux and roughness geometry

Above a thin layer near the roughness elements, Reynolds analogy is valid if the stability is near neutral, and it follows that Monın-Obukhov's similarity theory applies to the temperature profile as well if the shear temperature $\mathrm{T}_{*}$ of $\mathrm{Eq} 2117$ is given to

$$
\mathrm{T}_{*}=\frac{\mathrm{H}_{0}}{\kappa \mathrm{u}_{*}}
$$

A nondimensional temperature gradient $\phi_{\mathrm{H}}$ is then obtained

$$
\phi_{\mathrm{H}}\left(\frac{\mathrm{z}}{\mathrm{L}}\right)=\frac{\partial \theta}{\partial \mathrm{z}} \frac{\mathrm{zKu} \mathrm{u}_{*}}{\mathrm{H}_{0}}
$$

Because different conditions exist at large values of $z /|\mathbf{L}|$, we shall consider separately the temperature distributions in unstable and stable air

\section{Temperature Profiles in Unstable Shear Flow}

The temperature profile in the unstably stratified boundary layer has recently been well established through the careful measurements reported by Dyer $(1965,1967)$ which are shown in Fig 27 The plot shows a nondimensional temperature gradient 


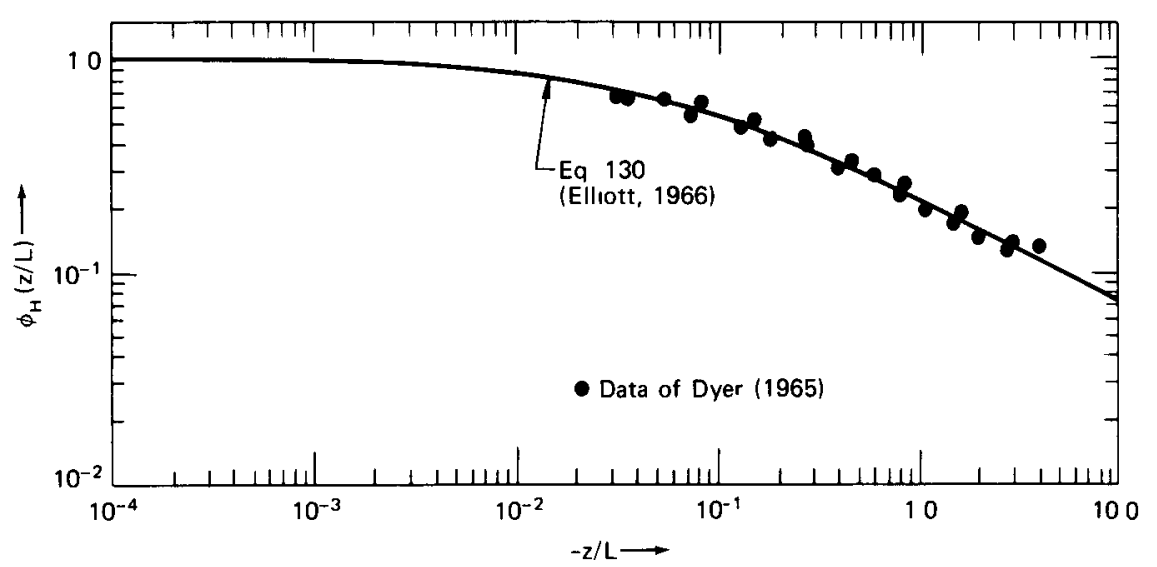

Fig. 2.7 Dimensionless temperature gradients in unstable flows. Field data from Dyer (1965), theoretical curve from Elliott (1966).

$\phi_{\mathrm{H}}(\mathrm{z} / \mathrm{L})$ as a function of $\mathrm{z} / \mathrm{L} . \phi_{\mathrm{H}}$ must be a constant equal to $1 / \mathrm{b}$ in the region where the temperature distribution is logarithmic, i.e., when $z / L \rightarrow 0$. For larger values of $-z / L$, a universal function relation is seen to exist between $\phi_{\mathrm{H}}$ and $-\mathrm{z} / \mathrm{L}$ in agreement with the similarity hypothesis of Monin and Obukhov

Dyer (1967) gave the following empirical equation obtained from this data

$$
\phi_{\mathrm{H}}=\left(1-15 \frac{\mathrm{Z}}{\mathrm{L}}\right)^{-055}
$$

which indicates a slope of about -0.5 for large values of $-z / L$ near 1 . Note that, if we combine the definitions of $\phi_{M}$ and $\mathrm{K}_{\mathrm{H}} / \mathrm{K}_{\mathrm{M}}$ given in Eqs. 2111 and 2115 , the result is

$$
\phi_{\mathrm{H}}=\frac{\mathrm{K}_{\mathrm{M}}}{\mathrm{K}_{\mathrm{H}}} \phi_{\mathrm{M}}=\frac{1}{\mathrm{~b}}\left(1-\beta \frac{\mathrm{z}}{\mathrm{L}}\right)^{-05}
$$

When $\beta=16$, Eqs 2.120 and 2121 are in agreement at $z / L=-1$ with $b=1.15$, thus it appears well justıfied to use Eqs $2.111,2115$, and 2121 for describing the unstably stratıfied surface layer so long as $-1<\mathrm{z} / \mathrm{L}<0$.

It has been argued by Priestley $(1955,1959)$ that at large values of $z / L$ the temperature profile should be independent of the shear stress. If this $1 \mathrm{~s}$ so, then Eq. 2.119 requires that $\phi_{\mathrm{H}}(\mathrm{z} / \mathrm{L}) \sim(\mathrm{z} / \mathrm{L})^{-1 / 3}$, and the profile has a $\mathrm{z}^{-\frac{4}{3}}$ dependency on the height. A constant nondimensional heat flux $\mathrm{H}_{*}$ can then be defined through the relation

$$
\mathrm{H}_{*}=\mathrm{H}_{0}\left(\frac{\mathrm{g}}{\mathrm{T}}\right)^{1 / 2}\left(\frac{\partial \theta}{\partial \mathrm{z}}\right)^{-3 / 2} \mathrm{z}^{-2}=\kappa^{2}\left(\frac{\mathrm{z}}{-\mathrm{L}}\right)^{-1 / 2} \phi_{\mathrm{H}}^{-\frac{3}{2}}
$$


The experimental results of Crawford (1965), Dyer (1967), and Swinbank and Dyer (1967) indicate that, in the region where the temperature distribution can be represented by Eq. $2122, \mathrm{H}_{*}$ has a value of about 115 to 1.3 .

If the analysis of Priestley actually applies to free convection, then Eq. 2.122 should be the asymptotic form of the temperature profile at free convection. It therefore comes as a surprise that the range at which $\phi_{\mathrm{H}}$ is proportional to $z^{-1 / 3}$ is not the asymptotic range but the midrange, with $\phi_{\mathrm{H}}$ changing to a steeper drop-off with $z /-L$ at larger values of $z /-L$, as follows from Eqs. 2.120 or 2.121 The reason for this discrepancy might stem from the fact that near the edge of the superadiabatic layer free convection takes place in organized motions of plumes or thermals, so that the Priestley assumption of a homogeneous turbulence structure is not valid The plumes that just start developing in the superadiabatic layer do not yet have the characteristics of fully developed plumes (to be described in Chap 3) because they first must be accelerated from essentially horizontal motion in the superadiabatic layer to an essentially vertical motion. A simple model of this flow has been proposed by Bryson (1955) and Ellott (1966), who considered a constant-temperature plume rising in a constant but lower temperature environment under the effect of buoyancy In the absence of friction, the constant buoyancy force per unit volume acting on the fluid particles in the plume gives rise to a constant acceleration and thus to a velocity that increases with height like $z^{1 / 2}$ Mass continuity of the plume then requires, in the absence of entrainment, that the area $\mathrm{A}$ of the plume decrease as $\mathrm{z}^{-1 / 2}$, consequently the temperature change with height is $\mathrm{dA} / \mathrm{dz}=\mathrm{d} \theta / \mathrm{dz} \sim \mathrm{z}^{-3 / 2}$, which should be valid near the transition zone between the superadiabatic and free-convection layers

With this asymptotic behavior of the temperature distribution valid at the edge of the superadiabatic layer, Elliott (1966) was able to construct a temperature profile for all of the superadiabatic layer by simply defining a nondımensional temperature profile equivalent to $\phi_{\mathrm{H}}$ of $\mathrm{Eq} 2.120$ for a temperature profile proportional to $\mathrm{z}^{-1 / 2}$

$$
\frac{\partial \theta}{\partial \mathrm{z}} \sim\left(\mathrm{u}_{*} \mathrm{H} \frac{\mathrm{T}}{\mathrm{g}}\right)^{1 / 2} \mathrm{z}^{-3 / 2}
$$

by assuming that the nondimensional heat flux $\mathrm{H}_{*}$ in $\mathrm{Eq} 2122$ is not constant but consists of two parts a part $\mathrm{H}_{*_{1}}$ corresponding to fully forced convection $\left(\phi_{\mathrm{H}}=1 / \mathrm{b}\right)$ and a part $\mathrm{H}_{* 2}$ corresponding to the temperature profile $\mathrm{Eq} 2.123$ For $b=1$, Eq 2.122 yields $\mathrm{H}_{*_{1}}=\kappa^{2}(\mathrm{z} /-\mathrm{L})^{-1 / 2}$, and Eq 2123 yields $\mathrm{H}_{*_{2}}=\alpha_{0}(\mathrm{z} /-\mathrm{L})$, where $\alpha_{0}$ is an empirical constant Consequently the nondimensional heat flux $\mathrm{H}_{*}$ to be used in Eq. 2122 becomes, with $b=1$

$$
\mathrm{H}_{*}=\kappa^{2}\left(\frac{\mathrm{z}}{-\mathrm{L}}\right)^{-1 / 2}\left[1+\alpha_{1}\left(\frac{\mathrm{z}}{-\mathrm{L}}\right)^{3 / 4}\right]
$$

The coefficients $\alpha_{0}$ and $\alpha_{1}$ are found by equating $\mathrm{H}_{*}$ to the constant $\mathrm{H}_{*}=13$ in the center of the Priestley regime. In this manner a value $\alpha_{0}=143$ was determined by Ellott, leading to a nondimensional temperature profile 


$$
\phi_{\mathrm{H}}\left(\frac{\mathrm{z}}{\mathrm{L}}\right)=\left[1+8.9\left(\frac{\mathrm{z}}{-\mathrm{L}}\right)^{3 / 4}\right]^{-2 / 3}
$$

This equation is in good agreement with the results of Dyer (or with Eq. 2.121) and thus lends support to the simple and appealing proposition of Elliott. It should, however, be slightly modified to allow for $b$ not equal to 1 .

Note that there exists a conceptual difficulty in accepting both Priestley's and Ellott's models It is hard to see why there should be a (Priestley) range in which the temperature distribution is independent of $\mathrm{u}_{*}$, which is embedded between two layers in which $u_{*}$ influences the motion. One would rather expect that the motion, once it has become independent of $\mathrm{u}_{*}$, will not become dependent again at higher elevations Consequently it is possible that a regime of the kind envisioned by Priestley does not exist

The lımit of validity of Eq. 2125 is difficult to determine. Beyond $z / L=-1$ the scatter of the data becomes excessive, no doubt because of the small gradients in temperatures. In fact, it will be shown in the next chapter that outside of the superadiabatic layer the temperature gradient actually may change sign, so that the analysis as presented here ceases to be valid outside of the superadiabatic layer, and it is not unreasonable to assume that the edge of the layer is located at about $\mathrm{z} / \mathrm{L}=-1$.

\section{Temperature Profiles in Stable Shear Flow}

Temperature profiles in stably stratified boundary layers have not often been reported, and the laboratory results of Arya and Plate (1969) reproduced in $F_{1} g 28$



Fig. 2.8 Dimensionless heat-flux coefficient as a function of stability in stable flows. Laboratory data of Arya and Plate (1969). 
may serve as a basis of discussion. The profiles are less accurate than the corresponding velocity data because of secondary currents observed in the laboratory at large $z / L$ values But there is little evidence to indicate that $\phi_{\mathrm{H}}$ is not a universal function of $\mathrm{z} / \mathrm{L}$, at least in the laboratory, and so long as the flow remans turbulent The laboratory data were taken at $\mathrm{z} / \mathrm{L}$ values too small for turbulence to be noticeably damped by buoyancy

The data show that a power law fits the $\phi_{\mathrm{H}}$ vs $\mathrm{z} / \mathrm{L}$ curve better over most of the range of $z / L$ values than the sımple log linear law of Monın and Obukhov. A law of the form

$$
\phi_{\mathrm{H}}=1+17 \frac{\mathrm{z}}{\mathrm{L}}
$$

which in a plot of $\theta$ vs $z / L$ appeared to give good fit to the temperature profiles is seen to show a different trend than the data, thus here again the conclusion must be reached that the log linear law is not a good enough approximation to actual profiles to be of much use

\section{The Heat Flux in Stratified Shear Flow}

As Owen and Thomson (1963) have shown, the heat flux is related to the temperature gradient across the roughness elements and the shear velocity $\mathrm{u}_{*}$ by

$$
\mathrm{H}_{0}=\mathrm{C}_{\mathrm{h}} \mathrm{u}_{*}\left(\mathrm{~T}_{\mathrm{w}}-\mathrm{T}_{\mathrm{h}}\right)
$$

where $T_{w}$ and $T_{h}$ are the temperatures of the ground and at the level $h$, respectively, $h$ is the thickness of the fluid layer which is directly distorted by the separation pattern of the roughness elements, and $C_{h}$ is a Stanton number, which can be expressed as a function of Reynolds and Prandtl numbers

$$
\mathrm{C}_{\mathrm{h}}=\frac{1}{\alpha_{1}}\left(\frac{\mathrm{u}_{*} \mathrm{~h}}{\nu}\right)^{-\mathrm{m}} \operatorname{Pr}^{-\mathrm{n}}
$$

Note that nether $h$ nor $T_{h}$ is known, so Eqs 2127 and 2128 are not useful unless these quantities can be determined. The length $h$ is a characteristic length of the roughness of the surface and is probably proportional to $z_{0}$. The exponents $m$ and $n$ as well as the coefficient $\alpha_{1}$ appear to be constant for a given roughness, in fact, there is some evidence that $\mathrm{m} \sim 0.5$ and $\mathrm{n} \sim 0.8$, independent of roughness, but $\alpha_{1}$ definitely depends on the roughness type The Stanton number therefore becomes

$$
\mathrm{C}_{\mathrm{h}}=\frac{1}{\beta_{1}}\left(\frac{\mathrm{u}_{*} \mathrm{Z}_{0}}{\nu}\right)^{-0}{ }^{-0} \operatorname{Pr}^{-0} 8
$$


where $\beta_{1}$ is a constant depending on the roughness. This relation is farly simple to test, and the constant $\beta_{1}$ can be determined from experimental data, provided that $T_{h}$ and $\mathrm{H}_{0}$ are known as well as the surface characteristics.

The value of $T_{h}$ is found from conditions in the flow above $z=h$. For $z>h$ Reynolds analogy is valid, and velocity and temperature profiles are of the forms discussed earlier. With $K_{H} / K_{M}=1.0$, it follows from Reynolds analogy that $\phi_{M}=\phi_{H}$, or

$$
\frac{\theta-\mathrm{T}_{\mathrm{h}}}{\mathrm{T}_{*}}=\kappa \frac{\overline{\mathrm{u}}-\overline{\mathrm{u}}_{\mathrm{h}}}{\mathrm{u}_{*}}
$$

which permits us to express the temperature profile in terms of the velocity profile. Elimination of $\mathrm{T}_{\mathrm{h}}$ from $\mathrm{Eq} 2130$ by means of $\mathrm{Eq} 2119$ (which implies a friction temperature $\mathrm{T} *=\mathrm{H}_{0} / k \mathrm{u}_{*}$ ) leads to

$$
\frac{\theta_{\mathrm{R}}-\mathrm{T}_{\mathrm{W}}}{\mathrm{T}_{*}}-\frac{\kappa}{\mathrm{C}_{\mathrm{h}}}=\kappa \frac{\overline{\mathrm{u}}_{\mathrm{R}}-\overline{\mathrm{u}}_{\mathrm{h}}}{\mathrm{u}_{*}}
$$

where the subscript $R$ denotes a reference case, say the conditions at $2 \mathrm{~m}$ above the ground. It is then possible to express a Stanton number $C_{s}$ based on reference conditions

$$
\mathrm{H}=\mathrm{C}_{\mathrm{s}} \mathrm{u}_{*}\left(\theta_{\mathrm{R}}-\mathrm{T}_{\mathrm{w}}\right)
$$

simply by replacing the temperature gradient with the velocity difference, or, since $\overline{\mathbf{u}}_{\mathrm{h}}$ $\ll \overline{\mathrm{u}}_{\mathrm{R}}$,

$$
\frac{1}{\mathrm{C}_{\mathrm{s}}}=\frac{\overline{\mathrm{u}}_{\mathrm{R}}}{\mathrm{u}_{*}}+\frac{1}{\mathrm{C}_{\mathrm{h}}}
$$

which is essentially the result given by Owen and Thomson (1963). Note that, to a good approximation, $\bar{u}_{R} / u_{*}$ is given from the $\log$ law to $(1 / \kappa) \ln \left(200 / z_{0}\right)$, where $z_{0}$ is the roughness length in centimeters, and $\mathrm{u}_{*} \mathrm{~h} / \nu$ can be written $\sim \mathrm{u}_{*} \mathrm{z}_{0} / \nu$. It therefore should be possible to express the Stanton number $C_{s}$ in the form

$$
\frac{1}{\mathrm{C}_{\mathrm{s}}}=\frac{1}{\kappa} \ln \frac{200}{\mathrm{z}_{0}}+\gamma\left(\frac{\mathrm{u}_{*} \mathrm{z}_{0}}{\nu}\right)^{\mathrm{p}}
$$

where the Prandtl-number dependency has been neglected because only air is considered, so that $\operatorname{Pr}=$ constant $=0.71$

This result has not yet been tested against atmospheric-field data, and presently avalable data do not contain enough information to calculate all parameters appearing 
in Eq 2134 In general, it can be expected that $\gamma$ and perhaps also $\mathrm{p}$ depend on the roughness type

A formulation for $C_{s}$ that does not depend on Reynolds analogy has been derived for unstable conditions by Leovy (1969) He specified a temperature profile which at some distance from the wall is given by Dyer's equation, Eq 2120 , and which very near the wall is linear and determined by molecular conditions only With the full specification of the temperature gradient, it is then possible to eliminate the heat flux from Eq 2131 by Eq 2119 and to obtain an expression for the Stanton number of the form

$$
C_{s}=f(B)
$$

where $B$ is defined to

$$
\mathrm{B}=\frac{1}{\mathrm{u}_{*}} \sqrt[3]{\kappa \mathrm{g}\left(\mathrm{T}_{\mathrm{R}}-\mathrm{T}_{\mathrm{w}}\right) \frac{1}{\mathrm{~T}_{\mathrm{A}}}}
$$

and $T_{A}$ is the average temperature across the superadiabatıc layer Note that $B$ can be calculated from bulk parameters of the flow and temperature field and that it is independent of the roughness characteristics of the surface The result obtaned by Leovy (1969) from data obtained during the Prarre Grass experıments (Lettau and Davidson, 1957) and by Vehrenkamp (1953) is shown in Fig 29, which generally

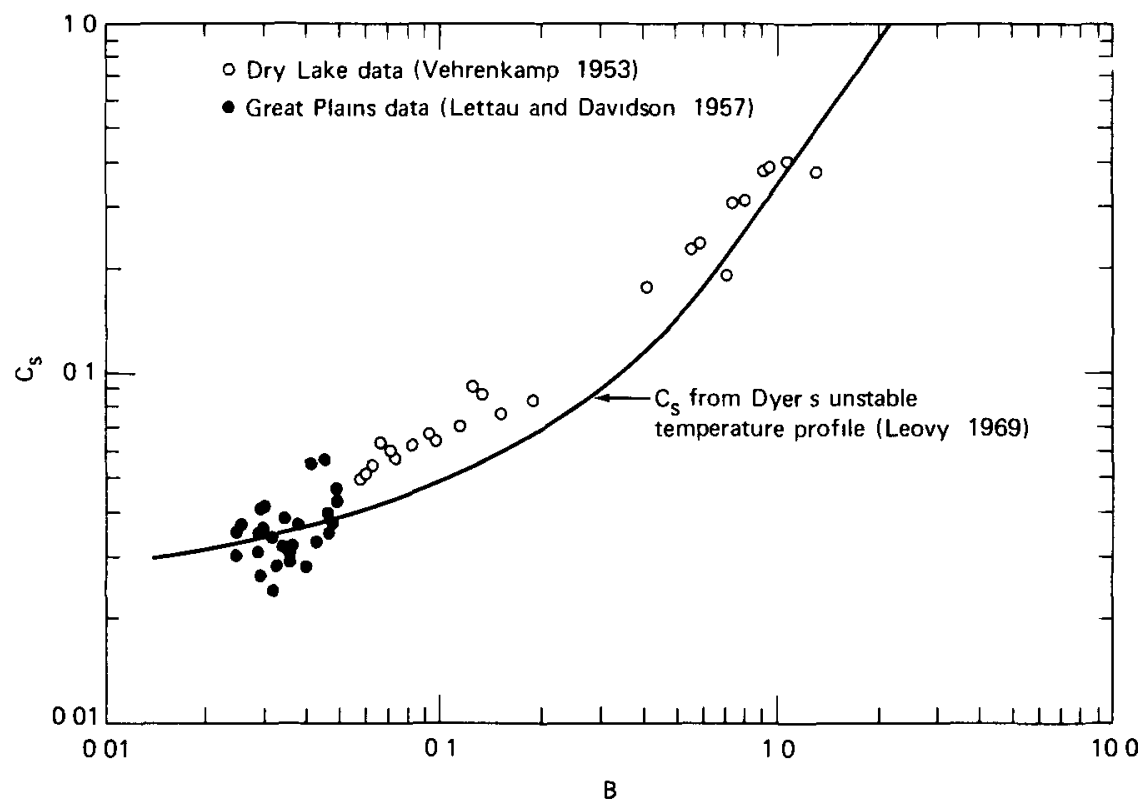

Fig. 2.9 Stanton number $C_{S}$ as a function of the index B Field data from Vehrenkamp (1953) and from the Great Plains Study [Lettau and Davidson (1957)] From Leovy (1969) 
shows the functional dependency rather well confirmed. Vehrenkamp's data that cover the widest range of $\mathrm{B}$ values correspond essentially to smooth surfaces for which the linear temperature profile at the ground assumed by Leovy is most likely to be valid. For rough surfaces, such as the Prairie Grass data, the functional dependency is not nearly as well confirmed. Further data are needed to illustrate whether Leovy's formulation is in fact sufficient; it does appear likely that the formulation of Owen and Thomson (1963) expressed by Eq. 2.134 is more appropriate, although at present less useful.

\section{CONCLUSIONS AND SUGGESTIONS FOR FUTURE RESEARCH}

In the discussions of this chapter (as well as in the free-convection chapter to follow), the complex structure of the stratified boundary layer has been brought out in a series of steady-state models each pertaining to a particular combination of given boundary conditions. As was pointed out in the introduction, none of the states that are observed is independent of what had happened before; and for quantitative results one should look at the whole moving picture of the dynamical development of the atmospheric boundary layer during the day instead of looking at a series of still pictures. Models in which the daily variation of temperature is considered (see the discussions in Chap. 8 of the book by Priestley, 1959 or in Chap. 4 of the book by Laikhtman, 1961) are at present highly formal and based not only on somewhat arbitrary assumptions about the structure of eddy diffusivities as a function of space and time but also on periodic inputs of heat by the solar radiation as driving force for the model. One possibility of useful research is therefore to put the detailed information on the planetary boundary layer that is currently available into a computer model for the development of the atmospheric boundary layer under the influence of large-scale circulation effects at given boundary conditions. If such a model is designed sufficiently flexible, it can be improved successively as more reliable information on the numerical parameters becomes available from field and laboratory studies. The most important theoretical purpose of the model would be to guide in the selection of experiments that need to be performed; its practical value lies in its ability to produce model environments and to aid in forecasting local conditions from large-scale forecasts.

The unsteady model is the ultimate goal, and one might well have to settle for intermediate steps for some time. Such an intermediate model would be that of an Ekman layer in stratified flows in which the nature of the flow field as described in this chapter and in Chap. 3 is taken into consideration. It is fairly evident that the eddy viscosity in a free-convection layer must have a different structure than in an inversion, and, for realistic models of Ekman layers, cognizance of this difference must be taken. In fact, the key problem of the stratified boundary layer in forced as well as free convection is the interrelation between buoyancy and momentum flux, and it is urgently required that this problem be investigated experimentally and analytically. 
Experimentally it appears that most important are measurements of the individual terms in the turbulent-energy equation, to establish energy balances both for the mean and for different frequency bands. Rehable measurements of turbulence in thermally stratıfied flows have become feasible only recently with the advent of on-line analog-to-digital converters, and most of the presently avallable experimental laboratory data are not reliable enough to evaluate such terms as gradients of triple correlation coefficients with some confidence. From such measurements it should be possible to evaluate the relative importance of the individual terms in energy balances, and, if experiments over wide enough ranges of variables can be made, such vital quantities as the critical Reynolds number at breakdown of turbulence and the dynamics of this process can be determined.

Of equal importance is a better understanding of the heat transfer from a rough surface by turbulent wind, at small values of $|\mathrm{L}|$, which in models of thermally stratified boundary layers yield one of the boundary conditions. The avalable equations like Eqs. 2.134 and 2.135 are at best tentative, and much work needs to be done before we understand the processes that cause heat to be transferred from the surface across roughness elements (which are possibly of different temperature than the ground) to the arr flow.

Finally one may list as challenging problems the determination of the structure of buoyancy-induced turbulence at interfaces between adjacent layers of different stratification, for example, in an environment where a superadiabatic layer is developing below an inversion or where a ground inversion builds up below a free-convection layer. Such problems are likely to be more profitably studied in density-stratified liquids, and the unsteadiness of the stratifications envisioned may present some difficult experimental problems. Nevertheless, these experiments are required if the mechanisms of turbulent transports in stratified planetary boundary layers are to be understood and if the empinical formulations of this chapter are to be put on a foundation of physical insight rather than on empincal-curve fittıngs.

\section{REFERENCES}

Arya, S P. S , 1968, Structure of Stably Stratıfied Turbulent Boundary Layer, Ph. D Dissertation, Colorado State University, Fort Collins, Colo.

—, and E. J. Plate, 1969, Modeling of the Stably Stratified Atmosphenc Boundary Layer, $J$ Atmos. Scl, 26: 656-665

_, and E. J Plate, 1970, Effect of Buoyancy Forces on the Turbulent Boundary Layer Above a Heated or Cooled Surface. Part I Mean Velocity and Temperature Profiles (to be published).

Ball, F. K., 1960, Control of Inversion Height by Surface Heating, Quart J. Roy. Meteorol. Soc., 86: $483-494$

Barad, M. L, 1963, An Examination of a Wind Profile Proposed by Swinbank, $J$ Appl. Meteorol., 2: 747-754.

Batchelor, G. K., 1953, The Conditions for Dynamical Similarity of Motions of a Frictionless, Perfect Gas Atmosphere, Quart. J. Roy. Meteorol. Soc., 79: 224-235.

Boussinesq, J., 1903, Theorie Analytique de Chaleur, Vol. 2, Gauther Villars, Pans.

Bryson, R. A., 1955, Convective Heat Transfer with Light Winds, Trans. Amer. Geophys. Union, 36: $209-212$, 
Businger, J. A., 1955, On the Structure of the Atmospheric Surface Layer, J. Meteorol., 12: 553-561.

1969, On Structure of the Unstable Atmospheric Boundary Layer (unpublished).

—, C. J. Wyngaard, Y. Izumı, and E. F. Bradley, 1970, Flux Profile Relationships in the Atmosphenc Surface Layer (to be published).

Calder, K. L., 1966, Concerning the Simularity Theory of A. S. Monin and A. M. Obukhov for the Turbulent Structure of the Thermally Stratified Surface Layer of the Atmosphere, Quart. J. Roy. Meteorol. Soc., 92: 141-146.

—, 1968, In Clanfication of the Equations of Shallow Layer Thermal Convection for a Compressible Fluid Based on the Boussinesq Approximation, Quart. J. Roy. Meteorol. Soc, 94: 88-92.

Chuang, H., and J. E. Cermak, 1967, Similarity of Thermally Stratified Shear Flows in the Laboratory and Atmosphere, Phys. Flutds, 10: Supplement Boundary Layers and Turbulence, pp. S255-S258.

Coantic, M., and B. Seguin (to be published, 1970).

Crawford, R. V., 1965, Moisture Transfer in Free and Forced Convection, Quart. J Roy. Meteorol. Soc., 91: 18-27.

Deacon, E. L., 1949, Vertical Diffusion in the Lowest Layer of the Atmosphere, Quart. J. Roy. Meteorol. Soc., 75: 89-103.

Dutton, J. A., and G. H. Fichtl, 1969, Approximate Equations of Motion for Gases and Liquids, $J$. Atmos Sct., 26: 241-254.

Dyer, A. J., 1965, The Flux Gradient Relation for Turbulent Heat Transfer in the Lower Atmosphere, Quart. J Roy. Meteorol. Soc., 91: 151-157.

_, 1967, The Turbulent Transport of Heat and Water Vapor in an Unstable Atmosphere, Quart J. Roy. Meteorol. Soc., 93: 501-508.

Ellott, W. P., 1964, The Height Variation of Vertical Heat Flux from the Ground, Quart. J. Roy. Meteorol. Soc., 90: 260-265.

—, 1966, Daytıme Temperature Profiles, J. Atmos. Sct., 23: 678-681.

Ellison, T. H., 1957, Turbulent Transport of Heat and Momentum from an Infinite Rough Plate, $J$. Fluid Mech., 2: 456-466.

— , and J. S. Turner, 1960, Mixing of Dense Fludd in a Turbulent Pipe Flow Pt. 2. Dependence of Transfer Coefficients on Local Stability, J. Flutd Mech., 8: 529-544.

Fleagle, R. J., and J. A. Businger, 1963, An Introduction to Atmospheric Physics, Academic Press, New York.

Gayevskaya, G. N., K. Y. Kondratiev, and K. E. Yakushevskaya, 1962, Radiative Heat Flux Divergence and Heat Regime in the Lowest Layer of the Atmosphere, Arch. Meteorol., Geophys. Bloklimatol, Ser. B, 12: 95-108.

Goody, R. M., 1964, Atmospheric Radiation, Oxford, Clarendon Press.

Gowen, R. A., and J. W. Smith, 1968, Turbulent Heat Transfer from Smooth and Rough Surfaces, Int. J. Heat Mass Transfer, 11: 1657-1673.

Holzman, B., 1943, The Influence of Stabulity on Evaporation, Ann. New York Acad. Scl., 44: 13-18.

Hınze, J., 1959, Turbulence, McGraw-Hull Book Company, Inc., New York, p. 66.

Kao, S. K., 1959, Turbulent Transfer in the Boundary Layer of a Stratified Flutd, J. Meteorol, 16: 497-503.

Kestın, J., and P. D. Richardson, 1963, Heat Transfer Across Turbulent Incompressible Boundary Layers, Int. J. Heat Mass Transfer, 6: 147-189.

Klug, W., 1967, Determination of Turbulent Fluxes of Heat and Momentum from the Wind Profile, Quart. J. Roy. Meteorol, Soc., 93: 101-104.

Lalkhtman, D. K., 1961, Physics of the Boundary Layer of the Atmosphere (English Translation, 1964), Israel Program for Scientific Translations, Sivan Press, Jerusalem.

Leovy, C. B., 1969, Bulk Transfer Coefficient for Heat Transfer, J. Geophys, Res., 74: 3313-3321. 
Lettau, H., and B Davidson, 1957, Exploring the Atmosphere's First Mile, Pergamon Press, Inc., New York.

Lumley, J., and H. Panofsky, 1964, The Structure of Atmosphenc Turbulence, John Wiley \& Sons, Inc., New York

McVehil, G. E., 1964, Wind and Temperature Profiles Near the Ground in Stable Stratification, Quart. J. Roy Meteorol Soc, 91: 136-146.

Monin, A. S., and A. M. Obukhov, 1954, Fundamental Regularities of Turbulent Agitation in the Ground Layer of the Atmosphere, Tr. Geofiz. Inst., Akad. Nauk. SSSR, Sb. Statei, 24: 163167.

Ogura, Y., 1952, Note on the Wind Velocity Profile in the Nonadiabatic Atmosphere, J. Meteorol. Soc Japan, 30: 329-342.

_ , and N. W. Phillips, 1962, Scale Analysis of Deep and Shallow Convection in the Atmosphere, J. Atmos Scl., 19: 173-179.

Owen, P. R., and W. R. Thomson, 1963, Heat Transfer Across Rough Surfaces, J. Flutd Mech., 15: 321.334

Pandolfo, J. P., 1966, Wind and Temperature Profiles for Constant Flux Boundary Layers in Lapse Conditions with a Vanable Eddy Conductivity to Eddy Viscosity Ratio, $J$ Atmos. Sct, 23: 495-502.

Panofsky, H. A., 1961, An Alternatıve Derivation of the Diabatic Wind Profile, Quart, J. Roy. Meteorol. Soc., 87: 109-113.

Plate, E. J., and C. W. Lin, 1966, Investigations of the Thermally Stratified Boundary Layer, Flu1d Mechanics, Paper No. 5, Colorado State University, Fort Collıns, Colo.

Priestley, C. H. B., 1955, Free and Forced Convection in the Atmosphere Near the Ground, Quart. J. Roy. Meteorol. Soc, 81: 139-143.

- 1959, Turbulent Transfer in the Lower Atmosphere, The University of Chicago Press.

Proudman, J., 1953, Dynamical Oceanography, Methuen, London.

Record, F. A., and H. E. Cramer, 1966, Turbulent Energy Dissipation Rates and Exchange Processes Above a Nonhomogeneous Surface, Quart. J. Roy. Meteorol. Soc., 92: 519-532.

Rider, N E., 1954, Eddy Diffusion of Momentum, Water Vapor, and Heat Near the Ground, Phll. Trans. Roy. Soc. London, Ser. A, 246: 481-501.

Rotta, J. C., 1962, Turbulent Boundary Layers in Incompressible Flow, in Progress in Aeronautical Sctences, Vol, 2, Pergamon Press, Inc., New York.

Schlichting, H, 1935, Turbulenz bel Warmeschichtung, Z, Angew. Math. Mech., 15: 313-338.

—, 1968, Boundary Layer Theory, McGraw-Hill Book Company, Inc., New York.

Spiegel, E. A., 1957, The Smoothing of Temperature Fluctuations by Radiative Transfer,

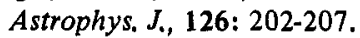

—, and G. Veronis, 1960, On the Boussinesq Approximation for a Compressible Flow, Astrophys, J., 131: 442-447.

Swunbank, W. C., 1955, An Experımental Study of Eddy Transports in the Lower Atmosphere, Technical Paper No. 2, C.S.I.R.O., Division of Meteorological Physics, Melbourne, Australia.

_, 1964, The Exponential Wind Profile, Quart. J. Roy. Meteorol. Soc., 90: 119-135.

—, and A. J. Dyer, 1967, An Experimental Study in Micrometeorology, Quart. J. Roy. Soc., 93: 495-500.

—, 1968, A Comparison Between Predictions of Dimensional Analysis for the Constant Flux Layer and Observations in Unstable Conditions, Quart. J. Roy. Meteorol. Soc., 94: 460-467.

Taylor, G. I., 1931, Effect of Variation in Density on the Stability of Superposed Streams of Flu1ds, Proc. Roy. Soc. (London) Ser. A., 132: 499-523.

Taylor, R. J., 1960, Simularity Theory in the Relations Between Fluxes and Gradients in the Lower Atmosphere, Quart. J. Roy. Meteorol. Soc., 86: 67-78.

Thorpe, S. A., 1969, Neutral E1gensolutions of the Stability Equation for Stratified Shear Flow, $J$. Flutd Mech., 36: 673-683. 
Townsend, A. A., 1958a, Turbulent Flow in a Stably Stratified Atmosphere, J. Fluid Mech., 3: 361-372.

_, 1958b, The Effects of Radiative Transfer on Turbulent Flow of a Stratified Fluid, J. Fluid Mech., 4: 361-375.

$\longrightarrow, 1967$, Wind and the Formation of Inversions, Atmos. Environ., 1: 173-175.

Vehrenkamp, J. E., 1953, Experimental Investigation of Heat Transfer at an Air-Earth Interface, Trans. Amer. Geophys. Union, 34: 22-30.

Webb, E. K., 1958, Vanishing Potential Temperature Gradients in Strong Convection, Quart. J. Roy. Meteorol. Soc., 84: 118-125.

_ , 1970, Profile Relationships: The Log-Linear Range, and Extension to Strong Stability, Quart. J. Roy. Meteorol. Soc., 96: 67-80.

Webster, C. A. G., 1964, An Experimental Study of Turbulence in a Density-Stratified Shear Flow, J. Fluid Mech., 19: 221-245.

Wood, J. D., 1969, On Richardson's Number as a Criterion for Laminar-Turbulent-Laminar Transition in the Ocean and the Atmosphere, Radio Sci., 4: 1289-1298.

Yamamoto, G., 1959, Theory of Turbulent Transfer in Nonneutral Conditions, J. Meteorol. Soc. Japan, 37: 60-69.

Zilitinkevich, S. S., D. L. Laikhtman, and A. S. Monin, 1967, Dynamics of the Atmospheric Boundary Layer, Izv. Akad. Nauk SSSR, Fiz. Atmos. Okeana, 3: 297-333 (English Translation, pp. 170-191).

, and D. V. Chalikov, 1968, Determining the Universal Wind Velocity and Temperature Profiles in the Atmospheric Boundary Layer, Izv. Akad. Nauk SSSR, Fiz. Atmos. Okeana, 4: 294-302. 
O 


\section{FREE-CONVECTION LAYER}

\section{INTRODUCTION}

In Chap. 2 the basic equations of motion and energy were derived for a thermally stratıfied boundary layer and were applied where the influence of the density stratification on the fluid motion is minor compared to that of the friction at the wall There exists a second asymptotic condition, that of free convection, in which fluid motions are dominantly driven by buoyancy The motions are initiated at the ground, which is at a higher temperature than the air above it In this chapter a discussion of this extreme case will be given. Fluid motions and heat transfer in free convection are described for dry air masses If the moisture content of the air is small and no changes in state are taking place, the conditions considered here can be applied directly to moist convection by replacing the sensible heat flux with the sum of sensible and latent heat fluxes and by assuming that the mean potential temperature profile remains unchanged.

Free convection occurs in the atmospheric boundary layer in two distinctly different situations. The first arises when there is no wind and the fluid is at rest The heat flux from the wall is by conduction if the viscous forces suffice to stabilize the fluid When the fluid becomes unstable, convection sets in and heat transfer is by mixing of moving hot fluid with the surroundings, first in a regular motion and then with higher heat fluxes, by a turbulent exchange process Following Priestley (1959), let us call this the case of "windless convection" A second type of free-convection layer can be found above a shear layer when the mean velocity gradients have been reduced to very small values. The heat flux superimposes a motion over this mean velocity field which essentially does not interact with the mean motion. The difference 
between this and windless convection is found mostly in the structure and nature of the turbulence; in addition to the buoyancy-induced turbulence, there may exist shear-generated turbulence which results from the shear flow near the ground and which penetrates the layers above.

For free convection the basic equations of motion and energy are much simpler than the equations of Chap. 2 It becomes possible to calculate heat transfer and velocity distributions. The first approximation to this problem, applicable to the determination of critical conditions for free convective motions, is obtained by a linearization of the equations. The velocity and temperature fields are superimposed over a state of pure conduction. However, if the flow and temperature field after the onset of convection are considered, closed-form solutions can no longer be obtained, analytical solutions are meaningless unless some of the nonlinearities are retained, and a set of approximations must be designed to make the problem tractable. We shall outline how this can be done. The purpose of this chapter, however, is to discuss what is known about the physical processes of free convection, and not to give detals of mathematical solutions. This purpose is best served when the free-convection problems are first stated in mathematical terms, after which the solutions and their physical significance are given. Steady convection, 1.e., the stationary convection state arrived at a long time after heat has been applied to the horizontal lower boundary, and unsteady (transient) convection, 1.e., the transient state developing during gradual application of heat, are treated separately and applied to laboratory and atmospheric conditions. A basic understanding of the fields induced by free convection is obtained from a discussion of laboratory experiments. Although laboratory experiments are not quantitatively useful for atmospheric applications, many of the qualitative features observed in the laboratory are also found in the atmosphere.

\section{BOUSSINESQ EQUATIONS FOR FREE CONVECTION}

In free convective motion the mean velocity gradients are zero at all times. All motions that exist are induced by buoyancy effects arising from conductive heating or cooling of the fluid. Considered here will be only the equations that result for a fluid bounded by planes perpendicular to the direction of gravity where heat is applied uniformly along each boundary plane. Then all mean derivatives with respect to $\mathrm{x}$ are zero, and substantial simplifications of the governing equations result. Assume that the reference potential temperature is constant and that energy dissipation can be neglected. The equations of continuity and of conservation of energy (Eqs. 2.23 and 2.28 in Chap. 2) then become

$$
\nabla \cdot \mathbf{v}=\left(\frac{\partial}{\partial \mathrm{t}}+\mathbf{v} \cdot \nabla\right) \frac{\mathrm{T}^{\prime}}{\mathrm{T}_{\mathrm{m}}}
$$

and

$$
\overline{\mathrm{k}} \nabla^{2} \frac{\mathrm{T}^{\prime}}{\mathrm{T}_{\mathrm{m}}}=\left(\frac{\partial}{\partial \mathrm{t}}+\mathrm{v} \cdot \nabla\right) \frac{\mathrm{T}^{\prime}}{\mathrm{T}_{\mathrm{m}}}
$$


where $\overline{\mathrm{k}}=\left(\mathrm{k} / \rho_{\mathrm{m}} \mathrm{c}_{\mathrm{p}}\right)$ For convenience the temperature is defined by

$$
\mathrm{T}^{\prime}=\overline{\mathrm{T}}(\mathrm{z}, \mathrm{t})+\theta(\mathrm{x}, \mathrm{y}, \mathrm{z}, \mathrm{t})
$$

where $\bar{T}$ is a mean (potential) temperature formed by averaging temperatures over a plane that is parallel to the ground $W_{1}$ th this expression, one obtains

$$
\mathbf{v} \cdot \nabla \mathrm{T}^{\prime}=\mathbf{v} \cdot \nabla \overline{\mathrm{T}}+\mathbf{v} \cdot \nabla \theta
$$

The second of the two terms on the right can be written

$$
\mathbf{v} \cdot \nabla \theta=\nabla \cdot \theta \mathbf{v}-\theta \nabla \cdot \mathbf{v}
$$

by using a well-known vector identity Furthermore, equating the left sides of Eqs 31 and 32 yields

$$
\mathbf{v} \cdot \nabla \theta=\nabla \cdot \theta \mathbf{v}-\theta \overline{\mathrm{k}} \nabla^{2} \frac{\mathrm{T}^{\prime}}{\mathrm{T}_{\mathrm{m}}}
$$

The first term in Eq 34 is simply

$$
v \cdot \nabla \overline{\mathrm{T}}=w \frac{\partial \overline{\mathrm{T}}}{\partial z}
$$

so that the energy equation becomes

$$
\frac{1}{\mathrm{~T}_{\mathrm{m}}}\left(\frac{\partial \mathrm{T}^{\prime}}{\partial \mathrm{t}}+\mathrm{w} \frac{\partial \overline{\mathrm{T}}}{\partial \mathrm{z}}+\nabla \cdot \theta \mathrm{v}\right)=\overline{\mathrm{k}}\left(\nabla^{2} \frac{\mathrm{T}^{\prime}}{\mathrm{T}_{\mathrm{m}}}\right)\left(1+\frac{\theta}{\mathrm{T}_{\mathrm{m}}}\right)
$$

The assumption $\theta / \mathrm{T}_{\mathrm{m}} \ll 1$ suggests that the second term on the right in Eq 35 can be neglected in Eq 38 This implies that either $\nabla \cdot \theta \mathrm{v}$ can also be neglected in Eq 38 or else, if $\nabla \cdot \theta \mathrm{v}$ is of the same magnitude as the other terms, that

$$
\mathbf{v} \cdot \nabla \theta \approx \nabla \cdot \theta \mathbf{v}
$$

If we retain the term $\nabla \cdot \theta \mathbf{v}$ and take the average over the horizontal plane $z=$ constant, the averaged energy equation becomes

$$
\left(\frac{\partial \overline{\mathrm{T}}}{\partial \mathrm{t}}+\overline{\mathrm{w}} \frac{\partial \overline{\mathrm{T}}}{\partial \mathrm{z}}+\frac{\partial\langle\theta \mathrm{w}\rangle}{\partial \mathrm{z}}\right)=\overline{\mathrm{k}} \nabla^{2} \overline{\mathrm{T}}
$$

and, when this is subtracted from Eq 38 , we also obtain, with Eq 39 ,

$$
\frac{\partial \theta}{\partial \mathrm{t}}+(\mathrm{w}-\overline{\mathrm{w}}) \frac{\partial \overline{\mathrm{T}}}{\partial \mathrm{z}}+\mathrm{v} \cdot \nabla \theta=-\frac{\partial\langle\theta \mathrm{w}\rangle}{\partial z}+\overline{\mathrm{k}} \nabla^{2} \theta
$$


The mean value, $\bar{w}$, of $w$ can arise only from the expansion of the air and is therefore very small and can be neglected Locally $w$ can be of substantial magnitude, as is particularly evident in situations with thermal elements

In Eq 311 the last two terms on the left-hand side-called the self-interaction terms-combine to form nonlinear terms representing the interaction of the fluctuations of temperature and velocity induced by the buoyant motion When the fluctuations are small, they are small to second order and can be neglected (Herning, 1963) To complete the set of equations, we must obtain an additional expression relating the temperature and velocities This 1 given by the momentum equation, whuch can be written

$$
\left(\frac{\partial}{\partial \mathrm{t}}-\nu \nabla^{2}\right) \nabla^{2} \mathbf{v}+\mathbf{R}=\frac{\mathrm{g}}{\mathrm{T}_{\mathrm{m}}} \nabla^{2} \mathrm{~T}^{\prime} \mathbf{k}-\frac{\mathrm{g}}{\mathrm{T}_{\mathrm{m}}} \operatorname{grad} \frac{\partial \mathrm{T}^{\prime}}{\partial \mathrm{z}}
$$

Equation 312 has been obtained by taking the double curl of Eq 229 in Chap 2 and by using the vector 1 dentities

$$
\begin{aligned}
& \text { curl curl } \mathbf{a}=-\nabla^{2} \mathbf{a}+\operatorname{grad}(\operatorname{div} \mathbf{a}) \\
& \text { curl grad } \mathbf{a}=0
\end{aligned}
$$

where a is a vector field and a is a scalar function Pressure is thus eliminated, and the nonlinear self-interaction terms of the velocity field are combined into the vector, $R$, defined by

$$
\mathbf{R}=+\operatorname{curl}[\operatorname{curl}(\mathbf{v} \cdot \nabla) \mathbf{v}]
$$

For small velocity components, terms quadratic in the velocity can be neglected if a small perturbation analysis is used in Eq 312 The resulting vertical component of Eq 312 is given by

$$
\left(\frac{\partial}{\partial \mathrm{t}}-\nu \nabla^{2}\right) \nabla^{2} \mathrm{w}=\frac{\mathrm{g}}{\mathrm{T}_{\mathrm{m}}}\left(\frac{\partial^{2} \theta}{\partial \mathrm{x}^{2}}+\frac{\partial^{2} \theta}{\partial \mathrm{y}^{2}}+\frac{\partial^{2} \theta}{\partial \mathrm{z}^{2}}\right)-\frac{\mathrm{g}}{\mathrm{T}_{\mathrm{m}}} \frac{\partial^{2} \mathrm{~T}^{\prime}}{\partial \mathrm{z}^{2}}
$$

which, with Eq 33 , reduces to

$$
\left(\frac{\partial}{\partial \mathrm{t}}-\nu \nabla^{2}\right) \nabla^{2} \mathrm{w}=\frac{\mathrm{g}}{\mathrm{T}_{\mathrm{m}}}\left(\frac{\partial^{2} \theta}{\partial \mathrm{x}^{2}}+\frac{\partial^{2} \theta}{\partial \mathrm{y}^{2}}\right)-\frac{\mathrm{g}}{\mathrm{T}_{\mathrm{m}}} \frac{\partial^{2} \overline{\mathrm{T}}}{\partial \mathrm{z}^{2}}
$$

To the same approximation, 1e, with self interaction terms neglected, Eq 311 becomes

$$
\left(\frac{\partial}{\partial \mathrm{t}}-\overline{\mathrm{k}} \nabla^{2}\right) \theta=-\mathrm{w} \frac{\partial \overline{\mathrm{T}}}{\partial \mathrm{z}}
$$


where the mean temperature gradient follows from Eq. 3.10 to

$$
\left(\frac{\partial}{\partial \mathrm{t}}-\overline{\mathrm{k}} \nabla^{2}\right) \overline{\mathrm{T}}=-\frac{\partial\langle\theta \mathrm{w}\rangle}{\partial z}
$$

In Eq. 318 the average of the fluctuation term has been retained to allow for a modification of the temperature distribution by the buoyancy-induced fluctuation field. Thus nonlınearity can be accounted for to some extent. Equations 316 to 3.18 have been used extensively in work on free convection

When a characteristic length $(h)$, a time scale $\left(t^{*}=h^{2} / \bar{k}\right)$, and a characteristic temperature $(\Delta T)$ are introduced, the nondimensional equations of motion and energy (Eqs. 316 and 317 ) become

$$
\begin{gathered}
\left(\frac{\partial}{\partial \mathrm{t}}-\nabla^{2}\right) \theta_{1}=-\mathrm{w}_{1} \frac{\partial \overline{\mathrm{T}}_{1}}{\partial \mathrm{z}} \\
\left(\frac{\partial}{\partial \mathrm{t}}-\nabla^{2}\right) \overline{\mathrm{T}}_{1}=-\frac{\partial}{\partial \mathrm{z}}\left\langle\mathrm{w}_{1} \theta_{1}\right\rangle \\
\left(\frac{1}{\operatorname{Pr}} \frac{\partial}{\partial \mathrm{t}}-\nabla^{2}\right) \nabla^{2} \mathrm{w}_{1}=\mathrm{Ra}\left[\left(\frac{\partial}{\partial \mathrm{x}_{1}^{2}}+\frac{\partial}{\partial \mathrm{y}_{1}^{2}}\right)-\frac{\partial}{\partial \mathrm{z}_{1}^{2}}\right]
\end{gathered}
$$

where the subscript 1 denotes nondimensional quantities and the nondimensional numbers, Pr and Ra, are the Prandtl number

$$
\operatorname{Pr}=\frac{\nu}{\overline{\mathrm{k}}}
$$

and the Rayleıgh number

$$
\operatorname{Ra}=\frac{\mathrm{g}}{\mathrm{T}_{\mathrm{m}}} \frac{\Delta \mathrm{T}}{v \overline{\mathrm{k}}} \mathrm{h}^{3}
$$

respectively, which are the only parameters governing conditions of free convection *

\section{STEADY CONVECTION IN A LAYER BETWEEN TWO PARALLEL AND HORIZONTAL PLATES}

When the temperature difference across a layer of fluid between two parallel and horizontal plates is increased in very small steps, heat transfer is by conduction only,

*In engineernng literature the Rayleigh number is usually replaced by the Grashoff number, Gr, defined by $\mathrm{Gr}=\mathrm{Ra} \cdot \operatorname{Pr}$. 
after a certain threshold value is exceeded, however, the fluid becomes unstable and motion ensues. The instability is triggered by small perturbations superimposed over the reference conductive state. There then exists a steady state at which the fluid is at incipient motion somewhere within the fluid. Conceptually this steady state is found by using very small step changes in temperature difference between the top and bottom plates and then waiting until the asymptotic state for $t \rightarrow \infty$ is attained. Analytically the steady state is discussed by assuming a steady state of conduction which at critical condition is not changed Pertinent equations for this condition are Eqs. 319 to 3.21 (with subscripts dropped)

$$
\begin{gathered}
\frac{\partial^{2} \overline{\mathrm{T}}}{\partial \mathrm{z}^{2}}=0 \\
\left(\frac{\partial}{\partial \mathrm{t}}-\nabla^{2}\right) \theta=-\mathrm{W} \frac{\partial \overline{\mathrm{T}}}{\partial \mathrm{z}} \\
\left(\frac{1}{\operatorname{Pr}} \frac{\partial}{\partial \mathrm{t}}-\nabla^{2}\right) \nabla^{2} \mathrm{w}=\operatorname{Ra}\left(\frac{\partial^{2} \theta}{\partial \mathrm{x}^{2}}-\frac{\partial^{2} \theta}{\partial \mathrm{y}^{2}}-\frac{\partial^{2} \overline{\mathrm{T}}}{\partial \mathrm{z}^{2}}\right)
\end{gathered}
$$

In these equations, only the vertical velocity, $w$, is given, other velocity components are found by using the continuity equation and the equation of motion. Equations 3.24 to 326 form, however, a closed set, and the stability problem depends on $w$ and $\theta$ only Therefore only these quantities will be discussed.

Equations 3.24 to 326 are nondimensional, and suitable reference scales must be chosen. For the problem of convection between parallel plates, the reference distance is the spacing, $h$, of the plates, and the temperature scale is the difference in temperature, $\Delta \mathrm{T}$, between the top and bottom plates.

\section{Heat Transfer by Conduction}

If the fluid is stable, heat transfer is by conduction only, even at critical conditions for the onset of convection. The mean temperature, $\bar{T}$, is then uncoupled from the fluctuating temperature field and is obtained by solving Eq. 324 . Integrating once yields the heat flux, $\mathrm{Nu}$

$$
\frac{\mathrm{d} \overline{\mathrm{T}}}{\mathrm{dz}}=\text { constant }=-1=-\mathrm{Nu}
$$

where the constant is found from the boundary conditions for the coordinate system shown in Fig. 3.1. The nondimensional heat flux is called the Nusselt number

$$
\mathrm{Nu}=+\frac{\rho c_{\mathrm{p}} \mathrm{H}_{0} \mathrm{~h}}{\mathrm{k} \Delta \mathrm{T}}=\frac{\mathrm{H}_{0} \mathrm{~h}}{\mathrm{k} \Delta \mathrm{T}}
$$


where $\mathrm{H}_{0}$ is the dimensional heat flux at the ground The temperature distribution is linear and in dimensional form is given by

$$
T(z)=T_{0}-\frac{\Delta T}{h} z
$$

and the dimensional heat flux, $\mathrm{H}$, is given by

$$
\mathrm{H}=-\overline{\mathrm{k}} \frac{\partial \overline{\mathrm{T}}}{\partial \mathrm{z}}=+\overline{\mathrm{k}} \frac{\Delta \overline{\mathrm{T}}}{\mathrm{h}}
$$

where $T_{0}$ is the temperature of the lower plate The linearity of the reference temperature profile substantially simplifies the stability problem, which arises when



F1g. 3.1 Definitions tor free convection between parallel plates

the conduction profile is disturbed by small perturbations Such perturbations are mostly caused by local nonuniformities in the wall temperature

\section{Instability of the Conduction State}

Instability in a layer of fluid that is heated from below was first studied theoretically by Rayleigh (1916) The restoring force counteracting the buoyancy is viscosity of the fluid, and instability occurs when the work done by buoyancy on the viscous layer is no longer balanced by viscous dissipation (Chandrasekhar, 1961) If the 
buoyancy work is smaller than the dissipation, the flund is stable, if it exceeds dissipation, the fluid is unstable to infinitesimal perturbations of temperature or velocity. The conditions at which the two terms are in exact balance correspond to one particular value of the Rayleigh number, which is the critical value $\mathrm{Ra}_{\mathrm{c}}$. Since viscous dissipation depends on the velocity gradients, which in turn are strongly affected by conditions at the boundaries, whereas the heat flux is dependent only on the heat added from below independent of the type of the boundary, the critical Ra number clearly depends on the boundary conditions. Two types of boundary conditions are of particular importance. The first is the condition for a rigid wall, where the no-slip requirement of a real fluid yields the boundary condition $v=0$. This condition is applicable to the atmosphere for windless convection near a smooth ground. The second condition is that of no stress acting on the fluid at the boundary This condition, which is introduced mainly for mathematical convenience, is obtained for a layer of fluid contained between two adjacent layers of other fluids, when all three layers move with the same mean velocity but are constrained so that the interface between the layers does not deform. Thus no shear stresses are transmitted, 1.e., $(\partial \mathrm{u} / \partial \mathrm{z})=(\partial \mathrm{v} / \partial \mathrm{z})=0$ and $w=0$ at the interface, and those conditions, together with the equation of continuity in the approximate solenoidal form (Eq 222 , Chap. 2) yield the boundary condition

$$
\left.\begin{array}{r}
w=0 \\
\frac{\partial^{2} w}{\partial z^{2}}=0
\end{array}\right\} z=0, h
$$

which is called the free-boundary condition

The stability problem for the free-boundary conditions is briefly outlined here, for the mathematical detals, reference is made to Chandrasekhar (1961) or to the basic paper by Pellew and Southwell (1940). With the linear conductive temperature profile, whose nondimensional slope is equal to -1 , Eqs. 325 and 3.26 become

$$
\left(\frac{\partial}{\partial \mathrm{t}}-\nabla^{2}\right) \theta=\mathrm{w}
$$

and

$$
\left(\frac{1}{\operatorname{Pr}} \frac{\partial}{\partial t}-\nabla^{2}\right) \nabla^{2} \mathrm{w}=\operatorname{Ra}\left(\frac{\partial^{2} \theta}{\partial \mathrm{x}^{2}}+\frac{\partial^{2} \theta}{\partial \mathrm{y}^{2}}\right)
$$

The stability analysis proceeds by assuming a Fourier-series solution for $w$ and $\theta$ and finding that value of Ra at which any of the amplitudes of the Fourier components are increasing in time. All real perturbations are found to grow exponentially with time for all Rayleıgh numbers corresponding to unstable gradients as a consequence of the 
linearity of the initial temperature profile The stability analysis is therefore reduced to finding the lowest Rayleigh number for which real amplitudes of spatial Fourier components are possible Needed is a solution of the nondimensional form (with $1=\sqrt{-1})$

$$
\begin{gathered}
w=\bar{w}(z) \exp 1\left(k_{x} x+k_{y} y\right) \\
\theta=\bar{\theta}(z) \exp 1\left(a_{x} x+a_{y} y\right)
\end{gathered}
$$

For convenience a horizontal wave number, a, is introduced and defined by

$$
a^{2}=\left(a_{x}^{2}+a_{y}^{2}\right)
$$

whose dimensional form is $\mathrm{kh}=\mathrm{a}$ Then the steady version of Eqs 332 and 333 reduces to

$$
\begin{gathered}
\left(D^{2}-a^{2}\right)^{2} \bar{w}=\operatorname{Ra} a^{2} \bar{\theta} \\
\left(D^{2}-a^{2}\right) \bar{\theta}=-\bar{w}
\end{gathered}
$$

where $\mathrm{D}$ is the operator, $\mathrm{d} / \mathrm{dz}$ Elımınation of $\bar{\theta}$ from these equations yields

$$
\left(D^{2}-a^{2}\right)^{3} \bar{w}=-R a a^{2} \bar{w}
$$

subject to the free-boundary conditions This is a sixth-order differential equation to be satisfied by three boundary conditions, each at $z=0$ and at $z=1$ This 1 s possible only for particular combinations of $\mathrm{Ra}$ and a For the free-boundary condition, the combinations of a and $\mathrm{Ra}$ which will satisfy $\mathrm{Eq} 339$ are simple to find because the solution satisfying the boundary conditions must be of the form

$$
\overline{\mathrm{w}}=\mathrm{A} \sin \pi \mathrm{nz} \quad \mathrm{n}=1,2,
$$

which has its lowest value for Ra for a given a at the smallest value of $n, 1 \mathrm{e}$, at $n=1$ The absolute minimum of $\mathrm{Ra}$ occurs at a particular wave number found from

$$
\frac{\partial \mathrm{Ra}}{\partial \mathrm{a}}=0
$$

to

$$
\mathrm{a}=\frac{\pi}{\sqrt{2}}
$$


corresponding to a wavelength

$$
\lambda=\frac{2 \pi}{\mathrm{a}} \mathrm{h}=2.82 \mathrm{~h}
$$

The minimum value of $R a=R a_{c}$ is 657.5. For $R a<R a_{c}$ the fluid is therefore free of motion, whereas for $\mathrm{Ra}>\mathrm{Ra}_{\mathrm{c}}$ motion occurs

For rigid boundaries the solution can no longer be found in terms of sine-Fourier series, but the solution in principle is obtained in the same manner. The characteristic equation yields a critical Rayleigh number of 1708 Both critical Rayleigh numbers are in good agreement with experiments. Only recently has an experiment been conducted, by Goldstein and Graham (1969), which was set up to meet the free-boundary conditions, and agreement within $10 \%$ with the theoretical critical Rayleigh number was found Rigld boundaries have been studied by many authors, for example, the series of experiments by Silveston (1958) gives excellent agreement with the theoretical value, as shown in Fig 32 After critical conditions are exceeded, the heat transfer is no longer by conduction, consequently the critical Rayleigh number can be inferred as that value of $\mathrm{Ra}$ at which $\mathrm{Nu}$ starts deviating from 1 . There is a sharp break observed in the curve $\mathrm{Nu}$ vs. $\mathrm{Ra} \sim 1700$ in $\mathrm{F}$ ig. 3.2.

Although Rayleigh numbers of the small magnitudes near critical are very unlikely to be encountered in the atmosphere, it is nevertheless worthwhile to remember the



Fig. 3.2 A comparison of Silveston's experimental measurements with theoretical predictions of heat transfer between infinite parallel plates $(O$, water, + , heptane, $\uparrow$, ethylene glycol, $\bullet$, slicone oll AK3, $\Delta$, silicone oll AK350, $\triangle$, a1r data of Mull and Reiher). [From I. Catton, Phystcs of Flunds, 9: 2521-2522 (1966) ] The famuly of curves below the data points represents the contributions of the individual modes to the total heat transfer. 
important conclusions obtained from the stability analysis and the pertinent experimental results (1) The wavelength of the critical disturbance is of the same order as the length scale of the geometry of the field, 1.e., near the maximum-length scale of the situation. (2) Buoyancy is thus seen to affect the large-scale motions more strongly than smaller scales (3) Steady convection is determined by a single nondimensional number, the Rayleigh number, however, if finite amplitude disturbances or unsteady convections are present, a second parameter enterning the processes is the Prandtl number. (4) The experiments of Silveston indicate that the change from conduction to convection is rather abrupt, the consequence of this observation, as exploited by Malkus $(1954 \mathrm{a}, \mathrm{b})$, is discussed in the next section

\section{Heat Transfer in Steady Free Convection}

When the critical Rayleigh number is exceeded, the convective currents set up in the fluid layer increase the rate of heat transfer. The stronger the heat input, the larger the convective currents, and on dimensional grounds one expects to find

$$
\mathrm{Nu}=\mathrm{f}(\mathrm{Ra}, \mathrm{Pr})
$$

where the Prandtl number has been added because it indicates the amount of heat released from the convective elements to the surroundings by conduction. An important experimental result found was that, for high Rayleigh and Prandtl numbers,

$$
\mathrm{Nu} \sim \mathrm{Ra}^{3}
$$

1.e., the heat transport is independent of the thickness, $h$, of the layer. This result cannot, of course be predicted from the linear model of Rayleigh, which ceases to be valid after convection has started. Solutions going beyond the critical Rayleigh number for onset of convection must incorporate some of the nonlinearities of the basic equations. Theories in which the equations of motion, Eqs. 3.10 to 3.12, have been partially linearized by neglecting quadratic terms in velocities, 1.e., the self-interaction terms, have been proposed by Malkus (1954b) and Herring $(1963,1964,1967)$. With these simplifications the governing equations are Eqs 3.16 to 3.18 for the temperature field and the vertical component of the velocity.

As was shown by Herring (1963), Eqs. 3.16 to 3.18 retain important features not only of the fluctuations arising just after the flow has become unstable but also remain valid to a first approximation for fully developed turbulent convection. The terms omitted correspond, for the turbulent convection problem, to the interactions of the turbulent motion with itself. Discarding them implies cutting off the hierarchy of turbulent moment equations, obtained by multiplying the dynamic equations by $v^{n}$ or $\theta^{\mathrm{n}}$, where $\mathrm{n}$ is some positive integer, after the second cumulant

Note that the only difference in Eqs 3.11 to 3.18 compared with Eqs. 3.24 to 3.26 hes in the term $(\partial\langle\theta w\rangle / \partial z)$ in Eq. 3.18 through which the mean temperature 
distribution is coupled to the fluctuations in temperature and velocity. This term is of fundamental importance. The mean temperature profile can no longer be considered as given, and a complete solution requires simultaneous solution of three instead of two equations. Furthermore, it has been pointed out that the growth of disturbances is exponential at all unstable Rayleigh numbers in a fluid with a linear mean temperature profile. Since the profiles are nonlinear, this is no longer true, and considerable complications arise. In fact, Eqs. 3.16 to 3.18 cannot be solved in closed form, and further approximations are required if solutions are desired. A great deal of effort has been made in recent years to obtain solutions from assumptions which simplify the basic equations and yet lead to predictions that agree satisfactorily with experiments.

The earliest, and one of the most successful attempts toward a solution of Eqs. 3.16 to 3.18 was made by Malkus (1954b). To calculate the heat flux and the convective motions, he started from his experimental observations (1954a) that the rate of change of heat flux with Rayleigh number appeared to show discontinuities. He reasoned that if these discontinuities were associated with the instabilities of subsequent eigenmodes of the convection problem, then for a particular Rayleigh number the nearest unstable eigenmode might set an upper bound on the harmonics of the disturbance motion, while the heat flux is in some manner related to the disturbance motion present. To put this concept on a quantitative basis, he postulated that at each Rayleigh number the heat flux (1) can only be down the gradient and (2), if averaged over the volume of fluid between the two plates, the heat flux must be a maximum compatible with the constraints of the problem. Thus, if the Rayleigh number permits instability of $n_{*}$ eigenmodes of the disturbance temperature, then the maximum heat flux is caused by all the modes in $n<n_{*}$. This model restricts the number of wave numbers contributing to the heat transfer, ${ }^{*}$ but not unreasonably, because disturbances with $n>n_{*}$ can appear only through nonlinear interactions and therefore are likely to make only small contributions to the heat flux. Malkus truncated the heat-flux spectrum at the harmonic $n_{0}=n_{*}-1$.

With these assumptions Malkus was able to obtain a solution with a number of results which are verifiable by experiments. In particular, he found that (1) the spectra of velocity and temperature fluctuations decrease with wave number as $n^{-1}$ and $n^{-0.5}$, respectively, (2) the kinetic energy of the fluctuating velocity is given in dimensional form by

$$
\left\langle\mathbf{v}^{\prime} \cdot \mathbf{v}^{\prime}\right\rangle \sim \frac{\overline{\mathrm{k}}}{\mathrm{h}^{2}} \mathrm{Ra}
$$

independent of the distance $z$ from the bottom plate, and (3) the mean temperature distribution is inversely proportional to $z$; at some distance from the plates and for $(\mathrm{z} / \mathrm{h}) \ll 1$,

*In the actual calculations, the $\mathrm{n}$ of the heat-flux modes did not correspond to the components of the stability problem. This inconsistency has been removed, to some extent, by Spiegel (1962). The reformulation of Spiegel did not lead to changes in the results. 


$$
\mathrm{T}\left(\frac{\mathrm{z}}{\mathrm{h}}\right)=\mathrm{T}\left(\frac{1}{2}\right)+\frac{\Delta \mathrm{T}}{2 \pi^{2}}\left(\frac{\mathrm{Rc}}{\mathrm{Ra}}\right)^{1 / 3}\left(\frac{\mathrm{z}}{\mathrm{h}}\right)^{1}
$$

where $\mathrm{Rc}$ is a constant equal to about 2533 The predictions of Malkus' theory have been verified to some extent by experiments Thomas and Townsend (1957) have shown that Eq 347 is in good agreement with experiments except for yrelding a flatter slope by a factor of about 15 The resulting equation (Eq 346 ) has been verified by experimental results of Malkus (1954b) and Deardorff and Willis (1967a) Figure 33 has been reproduced from the paper by Deardorff and Willis (1967a) to show the surprising agreement of theory and experiment Their data were for a highly turbulent flow and do not confirm Eq 347 , and the possibility that the agreement of experiments and Eq 346 is no more than a consequence of the dimensions of the problem cannot be ruled out

The heat transfer, expressed by Nusselt number $(\mathrm{Nu})$, obtained from the Malkus theory is (Malkus and Veronis, 1958)

$$
\mathrm{Nu}=1+\sum_{1=1}^{\infty} \mathrm{N}_{1}\left(1-\frac{\mathrm{Ra}_{\mathrm{c} 1}}{\mathrm{Ra}}\right) \delta\left(\mathrm{Ra}-\mathrm{Ra}_{\mathrm{Cl}_{1}}\right)
$$

where $\delta()$ is the Dirac delta function, $R_{a_{c 1}}$ is the critical Rayleigh number for instablity of the ith mode, and

$$
\mathrm{N}_{1}^{1}=\frac{\left(\left\langle\mathrm{w}_{1} \theta_{1}\right\rangle^{2}\right)_{\mathrm{AV}}}{\left(\left\langle\mathrm{w}_{1} \theta_{1}\right\rangle\right)_{\mathrm{AV}}^{2}}
$$

where the subscript $A V$ denotes averaging over the depth of the layer The calculations of Malkus (1954a) of $\mathrm{Ra}_{\mathrm{c} 1}$ and of Malkus and Veronis of $\left\langle\mathrm{w}_{1} \theta_{1}\right\rangle$ have been repeated by Catton (1966), and in the corrected form they yield excellent agreement with the experimental results of Silveston (1958) This agreement is seen in Fig 32, into which the theoretical curve based on the calculations of Catton (1966) has been drawn There seems thus to be little doubt that in as far as the heat flux is concerned, Malkus' model is quite realistic, although some recent experiments by Wills and Deardorff (1967a) indicate that the critical Rayleıgh numbers calculated by linear stability analysis are perhaps not really associated with the observed experimental breaks in the measured heat-transfer vs $\mathrm{Ra}$ number relation

Malkus' theory is noteworthy because it represents one of the few successful attempts to predict the structure of any type of nonhomogeneous turbulence It appears to work satisfactorily in convective motions because the turbulence induced by buoyancy is ordered and noninteracting at wave numbers associated with $n<n_{0}$, at which most of the interaction between heat flux and turbulence takes place Presumably the higher wave-number velocity fluctuations only smear out local discontinuities in the temperature but do not contribute to the heat flux The theory is therefore restricted to the large eddies of the turbulent motion, but most of the energy is associated with these, and for higher wave numbers it is usually not too 


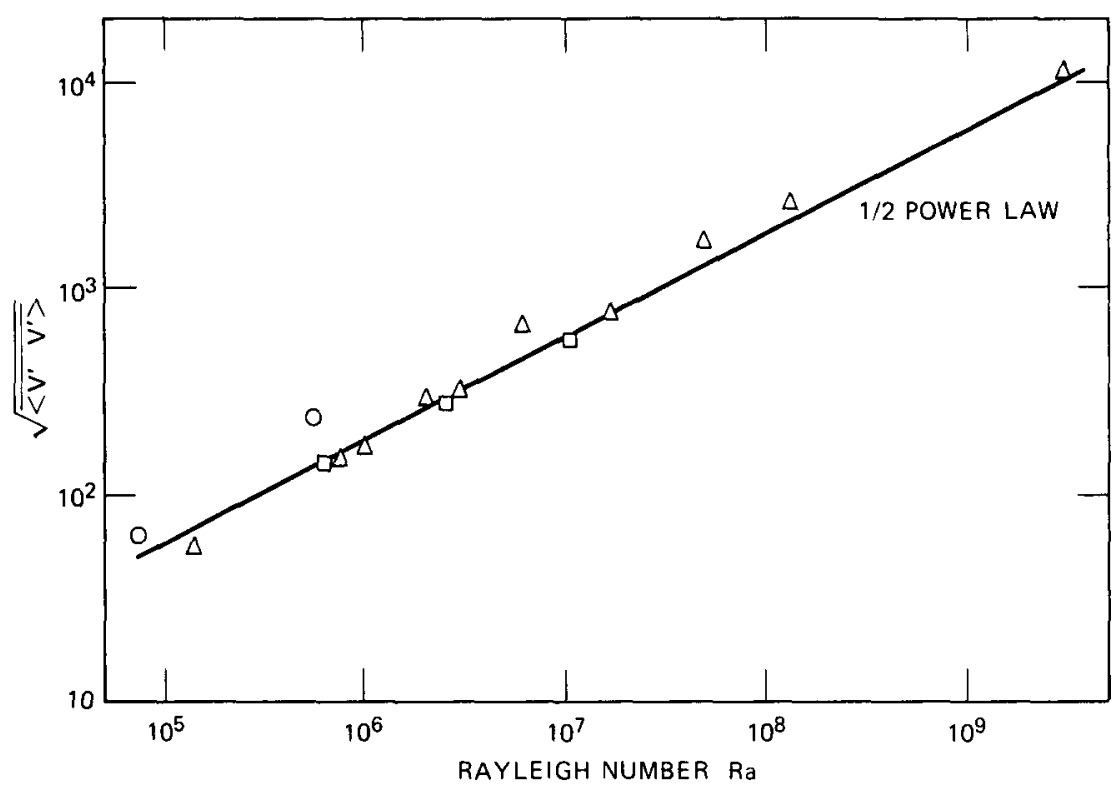

Fig. 3.3 Root-mean-square value of horizontally averaged vertical velocity fluctuations as a function of Rayleigh number. $O$, water [Malkus (1964b], $\operatorname{Pr}=7.0, \triangle$, acetone [Malkus (1954b)], $\operatorname{Pr}=3.7$, 口, aur [Deardorff and Wulls (1967a)], Pr $=0.71$. [From J. W. Deardorff and G. E. Wills, Journal of Flutd Mechantcs, 28: 675-704 (1967a).]

difficult to make reasonable predictions on the turbulence structure by invoking the concept of local isotropy. Thus, as long as the energy-containing eddies are noninteracting with each other and are separated from the energy-dissipating eddies of the turbulent motion, a model of the type envisioned by Malkus can be successful. But, when the eddies associated with the generation of turbulence interact strongly, the nonlinear velocity terms even in convection can no longer be neglected.

A theory simlar to that of Malkus (1954b) has been advanced by Herring (1963, 1964, 1967). His and Malkus' models have been compared in detall by Howard (1967). Herring also started with Eqs. 3.16 to 3.18, but, in contrast to Malkus, he assumed that a single disturbance wave number, $a$, is responsible for all the motion. This wave number, a, then is an additional parameter of the problem which has to be chosen arbitrarily, as long as the Rayleigh number is higher than the corresponding critical Rayleigh number, which is calculated from Eq. 3.37. On the other hand, Herring's model does not involve any other arbitrary assumptions. He shows that, if the combination of a (or for $\mathrm{Ra}<10^{6}$, two values of a) and $\mathrm{Ra}$ is chosen which yields marginal stability, then a reasonable value for the heat flux is obtained. In contrast to Malkus' assumption, this condition does not correspond exactly to maximum heat flux. The solution was obtained numerically by letting a small perturbation of nondimensional wave number a evolve in time until an asymptotic state is reached. Herring (1963) considers free boundaries, and Herring (1964), rigid boundaries and 
Prandtl number, $\operatorname{Pr} \rightarrow \infty$ The free-boundary case was again treated for large $\mathrm{Ra}$ and $\mathrm{Pr}$ (Herring, 1966) as a steady-state problem, with the result again that for $\mathrm{Ra}>10^{8}$ the Nusselt number is proportional to $\mathrm{Ra}^{\text {t/3 }}$, with a factor of proportionality which is somewhat higher than the experimentally observed value of Silveston. For large $\mathrm{Pr}$, Herrıng (1966) gives

$$
\mathrm{Nu}=0.115 \mathrm{Ra}^{1 / 3}
$$

and reports the experimental results of

$$
\mathrm{Nu}=(0.09 \div 0.134) \mathrm{Ra}^{1 / 3}
$$

where the low value corresponds to the data of Silveston (1958) and the high value to data of Globe and Dropkin (1959).

Herring's results for the temperature profile for the most important rigid-boundary case show the interesting result that the flow essentially is separated into two very distinct regions, as shown in Fig. 3.4a (Herring, 1964). Near the walls the temperature gradient changes rapidly from its value for conductive heat flux to zero. The thickness of the layer in which this change takes place decreases with increasing Rayleigh numbers. In the center portion the temperature is almost constant and equal to the average temperature of the two walls As shown in Fig. 3.4b, both these features have also been observed by Deardorff and Willis (1967a) in laboratory experiments. The convection process is thus seen to be of boundary-layer character. This result is in contrast to Malkus' profile, Eq. 3.46. However, because of the assumptions made in deriving 1 t, the latter should be valid only in the transition region between the constant temperature and the gradient layers, and a direct comparison with Herring's results in regions where both profiles are valid has not been attempted.

Other methods of predicting the heat transfer of the free-convection problem are due to Howard (1963) and Kraichnan (1962). Howard applied the maximum heat-flux assumption of Malkus (1954b) to find upper bounds on the heat transfer subject to constraints imposed by integrals obtained from Eqs. 3.25 and 3.26 and the boundary conditions (see also Busse, 1969) In Kraichnan's paper the effect of the Prandtl number is considered, and heat-flux predictions based on a mixing-length-type argument are made. Herrıng (1969) has applied yet another method to the solution of this problem, the direct interaction hypothesis of Kraichnan.

Further models based on the structure of the thermal elements which produce the convective motion will be considered later Since regular motions pertaining to convection at low Rayleigh numbers are no longer found in highly turbulent convection, separate considerations must first be given to the structure of these elements. Models based on the assumption that the space between the plates is filled by convective cells of known characteristics have also led to heat-transfer predictions in reasonable agreement with experiments, as, for example, in the paper by Wesseling (1969). However, in highly turbulent flow that is unbounded from above, the thermal elements do not completely fill the convective layer. 
It is perhaps surprising that the problem of the convection process between two plates has been treated by so many different techniques. The reason for this lies in the fundamental nature of this flow The flow is simple because no mean velocity field
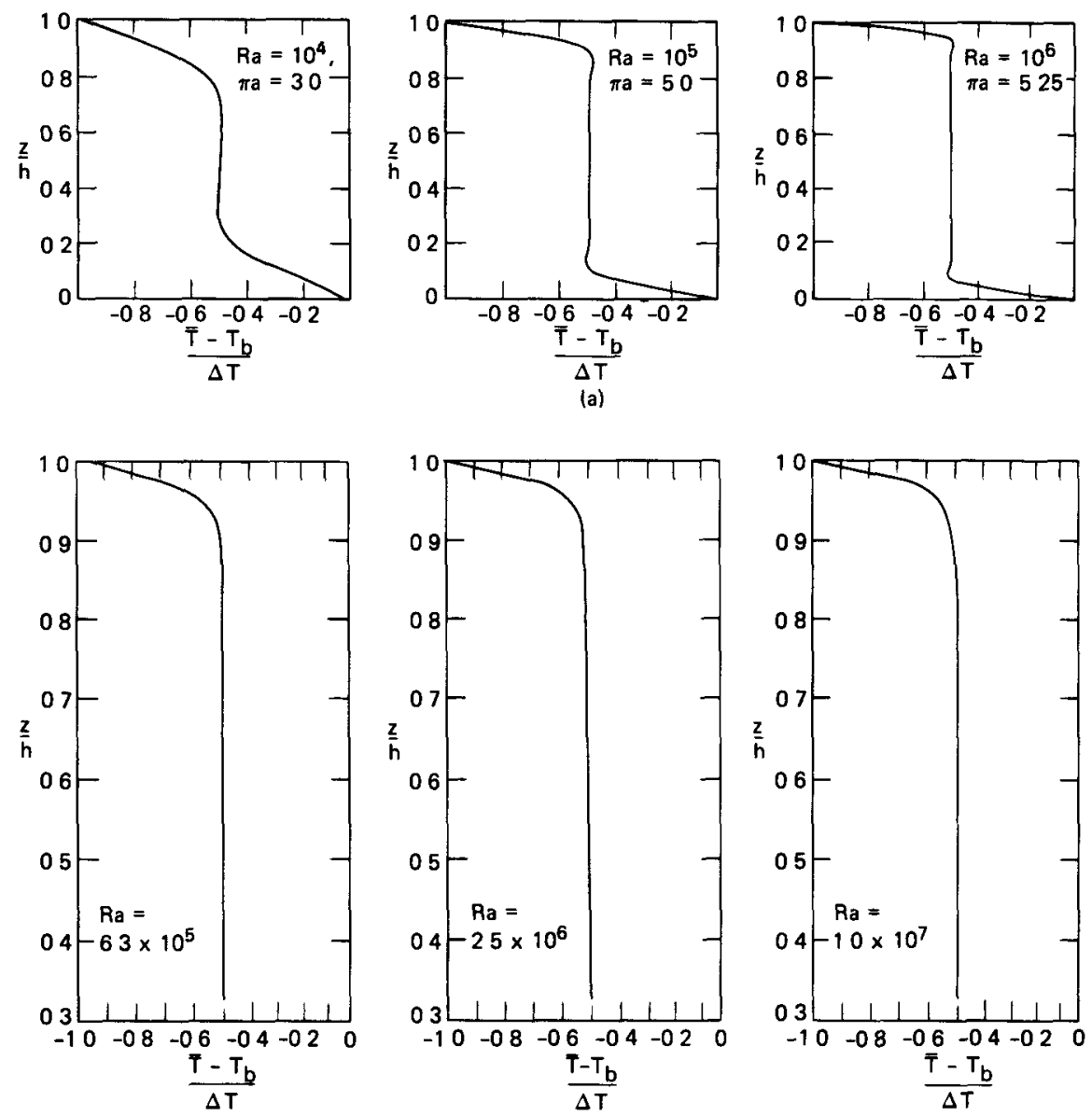

(b)

Fig. 3.4 (a) Vertical profiles of horizontally averaged temperatures at three Rayletgh numbers, calculations by Herring (1964) (b) Vertical profiles of horizontally averaged temperatures at three Rayleigh numbers, laboratory results of Deardorff and Wills (1967a).

exists which might add to the production of turbulence, and all motions are induced by the directed force of buoyancy Yet, the flow exhibits many characteristics of turbulence and is therefore an ideal testing ground for models of turbulence Any model that fals the test of predicting the free-convection model is unlikely to yield reasonable predictions in more complicated nonhomogeneous turbulent flows 
STEADY CONVECTION IN AN INFINITE-THICKNESS LAYER

In the convection models considered in the previous section, the length scale of the motion was set by the distance between the two plates, or the thickness of the layer The convective elements that made up the motion were found to have dimensions of the order of the characteristic length. But, when the layer is very thick, it is possible that the thickness of the layer no longer scales the size of the thermal elements. Instead, the buoyant motion sets its own scale, which can be found from dimensional analysis. We may assume that the heat flux must depend on the properties that drive the turbulent motion, but not on the molecular transfer coefficients $\vec{k}$ and $\mu$ Thus the only length scale that is descriptive of the geometry of the problem must be the distance, $z$, whereas the other characteristics are the effective gravity $(\mathrm{g} / \mathrm{T})$ [gravity multiplied by the coefficient of thermal expansion, which for an ideal gas is $(1 / T)]$, the density, the specific heat, and the temperature gradient. It follows readily that

$$
H=\rho c_{p}\left(\frac{g}{T}\right)^{\alpha} z^{\beta}\left|\frac{\partial T}{\partial z}\right|^{\gamma}
$$

where the exponents $\alpha, \beta$, and $\gamma$ must be chosen so that the three terms together have the dimension length times temperature over time. The only combination that gives these dimensions results in

$$
H \sim \rho c_{p}\left(\frac{g}{T}\right)^{1 / 2} \quad\left(-\frac{\partial T}{\partial z}\right)^{3 / 2} z^{2}
$$

For a steady convective situation in thermodynamic equilibrium, neglecting the heat gained by dissipation, the heat flux must be constant, then Eq. 3.53 can be inverted to yield the temperature distribution,

$$
\frac{\partial T}{\partial z}=-r\left(\frac{H}{\rho c_{p}}\right)^{2 / 3} \quad\left(\frac{T}{g}\right)^{1 / 3} z^{-4 / 3}
$$

where $r$ is a factor of proportionality. This equation has first been derived on the basis of mixing-length arguments by Prandtl (1932) The dimensional-analysis derivation was given by Priestley (see Priestley, 1959).

Equation 3.54 can be interpreted as a similarity profile for the temperature distribution of the form

$$
\frac{\mathrm{L}}{\mathrm{T}_{\mathrm{z}}} \frac{\partial \mathrm{T}}{\partial \mathrm{z}}=-\mathrm{r}\left(\frac{\mathrm{z}}{\mathrm{L}}\right)^{-\mathrm{s} / \mathrm{s}}
$$

where $L$ is a reference length $=(1 / g)\left(H / \rho c_{p} T_{a}\right)^{2}$ and $T_{a}$ is a reference temperature. The length, $L$, has no dynamic significance unless $r$ is independent of $(z / L)$, in which case it 
derives its physical content from Eq. 3.53. Townsend (1962) has argued that this form of the free-convection profile requires for its existence that some mean horizontal shear exists which might have the effect of homogenizing the local turbulence structure that otherwise would be in nonordered form. Deardorff and Willis (1967b) have examined laboratory data of their own and the data of Thomas and Townsend (1957) and arrived at the conclusion that the $\mathrm{z}^{-1 / 3}$ law of Priestley as well as the $\mathrm{z}^{-1}$ law of Malkus for the temperature distribution is likely to be confined to a thin layer only. They argued that the absence of a $\mathrm{z}^{-1 / 3}$ range for the temperature distribution in the laboratory data might be a scale effect, set by the size of the experimental equipment, and suggested a critical experiment at an appreciably larger scale than previous laboratory experiments to settle this point. This experiment has, however, not been performed, and therefore the relevancy of the laboratory experiments to the atmospheric-surface layer under conditions of windless free convections has not been established. This point will be discussed again later.

\section{UNSTEADY \\ FREE CONVECTION}

The stability problem of Rayleigh outlined earlier reduced to one of steady conditions because the temperature gradient of the basic Rayleigh problem was constant. The situation changes quite drastically when the requirement of an initially linear temperature profile is dropped. A nonlinear temperature profile arises when the temperature of one of the walls is changed. The resulting local change in heat flux progresses into the interior of the fluid and thereby heats (or cools) the fluid. Locally there might exist a Rayleigh number that exceeds a critical value for the onset of instability. Directly at the wall, instabilities are prevented by the wall. However, once the heat flux has penetrated the conductive region at the wall, it can produce local instabilities in spite of the fact that the Rayleigh number associated with average conditions might be lower than that required for instability. This behavior is quite evident in the following rather common situation. Consider a stably stratified fluid of air between two plates, where the stability is maintained by keeping the lower plates at a lower temperature than the upper plate. At some time $t_{0}$, the lower plate is brought to the same temperature as the upper plate. The Rayleigh number has then changed from a negative value to zero. Locally, however, near the bottom plate the temperature gradient can be very steep due to the finite time required for heating of the layer. Obviously, whether instability sets in or not depends on the speed of applying the temperature change as compared to the adjustment time constant of the fluid. Evidently, if the temperature change is gradual enough, the fluid never is set in motion, and the adjustment is by conduction only.

The initial-value problem of the development of a convective layer with time has been discussed for the linearized (Rayleigh) problem by Foster (1965), who studied both a linear increase (case $B$ ) of the temperature and the abrupt change (case $A$ ) in 
temperature of the bottom plate He studied the system of Eqs 319 to 321 by a Fourier analysis of the velocity and temperature field after removing the nonlinear term in Eq 320 by the small-perturbation procedure

The relevant Rayleigh number for case B is

$$
\mathrm{Ra}=\frac{\mathrm{gBh}^{5}}{\mathrm{~T}^{2} \nu}
$$

where

$$
\frac{B h^{2}}{\bar{k}}=\Delta \mathrm{T}
$$

1s the temperature scale factor, $1 \mathrm{e}, \mathrm{T}_{\mathrm{B}}=\mathrm{T}_{0}+\mathrm{Bt}$, where $\mathrm{T}_{\mathrm{B}}$ is the bottom temperature and $T_{0}$ is the temperature of the bottom plate at $t$ ime $t=0$ For case $A$ the Rayleigh number is formed in the usual manner with $\Delta \mathrm{T}$ equal to the step change in temperature Foster restricted his investigation to the free-boundary case, and an initial input of a white noise distribution of finite-amphitude $w$ disturbance modes The computer results of Foster showed the expected decay of disturbances at very short times But soon the disturbances started to grow superexponentially Critical times for the onset of instablity were calculated using the criteria that the root-mean-square (rms) value, $\overline{\mathrm{w}}$, of $\mathrm{w}$ had reached a multiple of its initial values The solution depends on the Prandtl numbers of the fluid, becoming independent of Pr only for very large Pr values Results of Foster (1965b) are shown in Fig 35 For water, Foster (1965a) compared the theory with experimental data of Spangenberg and Rowland (1961), who determined the tıme that elapsed before a layer of water which was made unstable by cooling from above by evaporation started to turn over Additional experiments, also indicated in $F_{1} g 35$, with a similar apparatus covering a wider range of Rayleigh numbers were performed by Foster (1965b) These experiments correspond approximately to the fixed-boundary case with linear change in temperature at the surface

Interestingly Foster found in both cases $\mathrm{A}$ and $\mathrm{B}$ that the critical time for instability to occur after the beginning of the experiment was independent of the height, $h$, and so was the wavelength, $\lambda$, of the instability, $1 \mathrm{e},\left(\overline{\mathrm{k}} \mathrm{t} / \mathrm{h}^{2}\right) \sim \mathrm{Ra}{ }^{2 / 3}$ and $(\lambda / \mathrm{h}) \sim \mathrm{Ra}^{-1 / 3}$ for the step change in temperature and $\left(\overline{\mathrm{kt}} / \mathrm{h}^{2}\right) \sim \mathrm{Ra}^{-2 / 5}$ and $(\lambda / \mathrm{h}) \sim \mathrm{Ra}^{1 / 5}$ for the linear temperature change It follows that the thickness of the layer which is affected by temperature changes is proportional to the square root of time

The latter result can be inferred directly by inspecting the conservation-of-energy equation for infinitesimal disturbances of temperature As long as the region between the plates is effective in damping infinitesimal disturbances, Eq 320 becomes the heat-conduction equation

$$
\frac{\partial T}{\partial t}=k \frac{\partial^{2} T}{\partial z^{2}}
$$




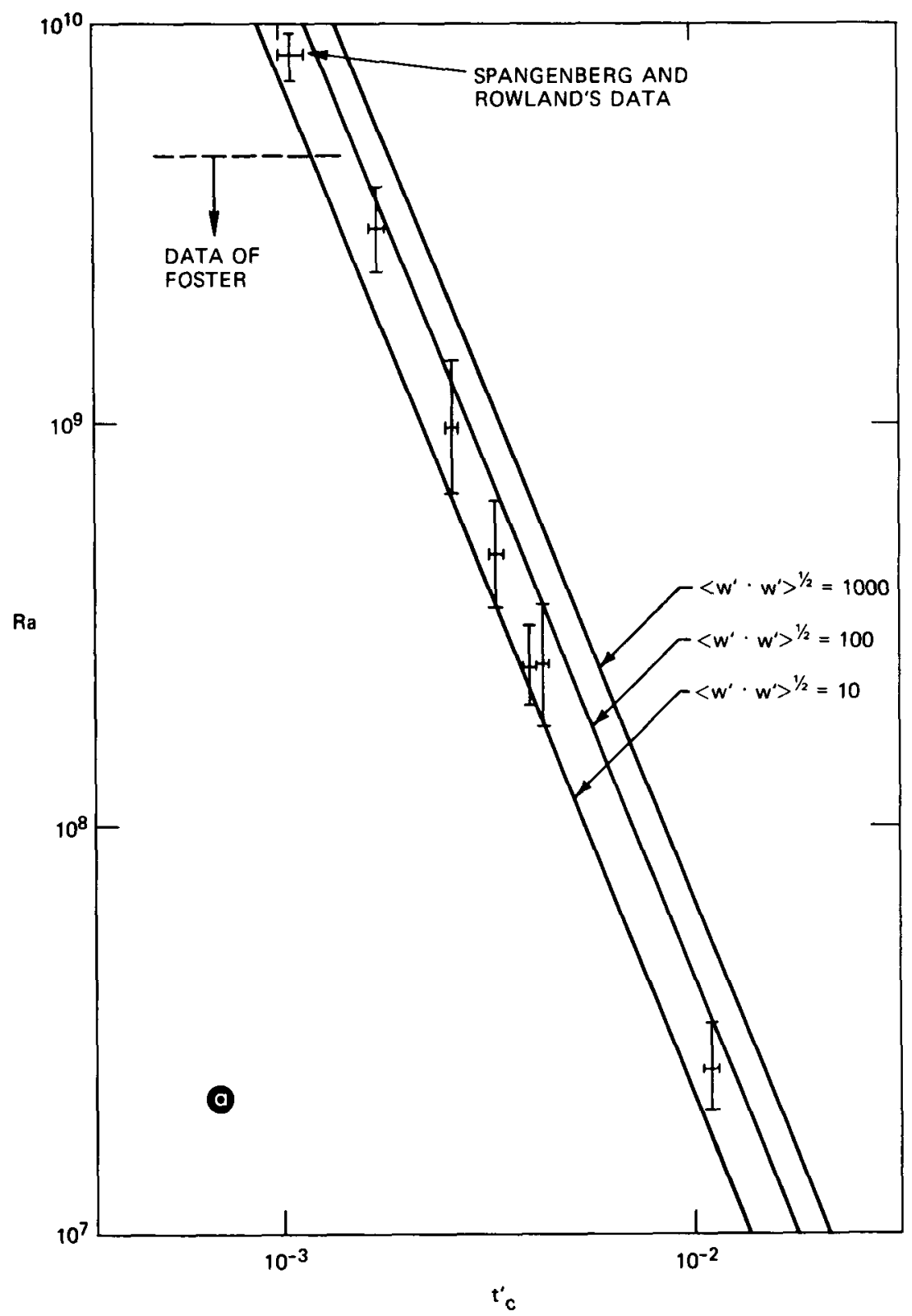

Fig. 3.5 (See facing page for legend.) 


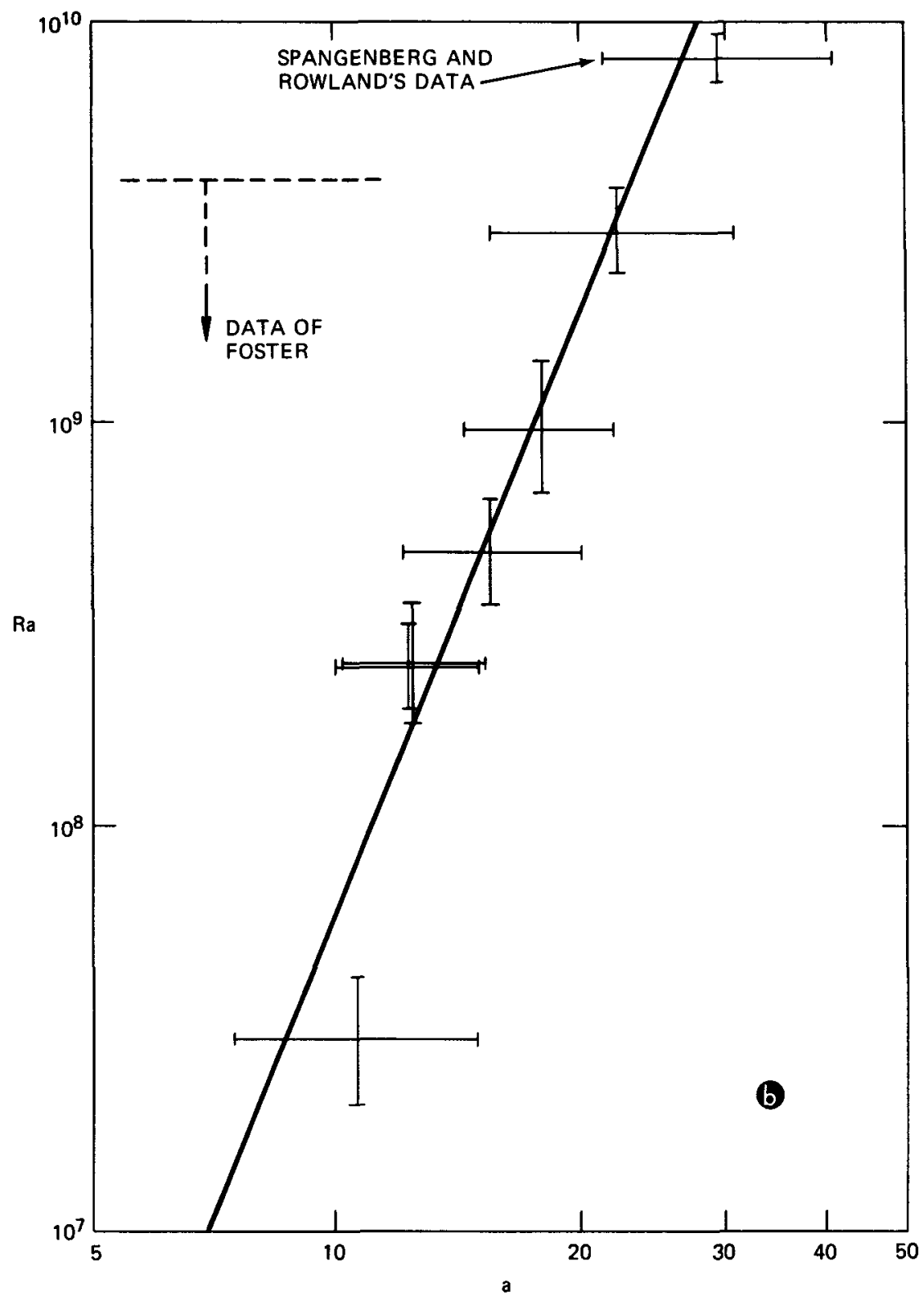

Fig. 3.5 (a) Experimental values of Rayleigh number vs. dimensionless nominal critical time. Theoretical curves for a linear decrease in surface temperature; $\operatorname{Pr}=5.8$. $(b)$ Experimental values of Rayleigh number vs. dimensionless critical wave number. Theoretical curve for a linear decrease in surface temperature; $\operatorname{Pr}=5.8$. [Adapted from Foster (1964a).] 
which for a step temperature change by $\Delta \mathrm{T}$ at time $\mathrm{t}=0$ has the well-known solution

$$
\overline{\mathrm{T}}-\overline{\mathrm{T}}_{\mathrm{wall}}=\frac{\Delta \mathrm{T}}{\sqrt{\pi}} \int_{\xi}^{\infty} \mathrm{e}^{-\xi^{2} \mathrm{~d} \xi}
$$

where $\xi$ is the dimensionless distance coordinate:

$$
\xi=\frac{\mathrm{z}}{2 \sqrt{\mathrm{k} t}}
$$

The characteristic length scale of the temperature profile is $2 \sqrt{\mathrm{kt}}$, in qualitative agreement with Foster's results. A further consequence of the heat-conduction equation is that disturbances that generate at the wall decrease in amplitude proportional to $\mathrm{e}^{-\mathrm{n}}{ }^{2} \overline{\mathrm{k}} \mathrm{t}$, where $\mathrm{n}$ is the (dimensional) wave number of the disturbance. As Elder (1968) pointed out, this implies that the conductive layer acts as a low-pass filter toward disturbances originating at the wall. The convective layer is thus subjected only to relatively low disturbance wave numbers, which indicates a reason for the existence of comparatively large convective elements.

The stability problem of Foster has also been considered as a problem of steady conduction with a nonlinear temperature profile by assuming the time change of the mean temperature distribution to be so slow that it had no effect on the stability problem (Lick, 1965; and Currie, 1967). The limitations of this assumption were discussed by Robinson (1967).

The development of the structure of the temperature and flow field during unsteady free convection has been investigated by Elder (1968) both in a laboratory and numerically by using the linearized equations on which Foster's studies were based. In contrast to the study of Foster, Elder (1968) did not consider Fourier modes of the disturbance velocities but instead traced the development of the flow field as a whole by solving the equations of the problem by means of finite-difference techniques on a computer. His numerical experiments were paralleled by laboratory experiments with flow visualization of the thermal elements. Both studies confirmed that the critical time for a disturbance to occur varied with $\mathrm{Ra}^{-2 / 3}$ and was independent of the layer thickness. The process of convective transport was found to take place in well-defined stages. Directly at the wall the no-slip condition constrains the motion, setting up local velocity gradients and shear stresses; at short times, only conduction takes place within a thin layer, which is the conductive sublayer (called protosublayer by Elder). With progress in time, local accumulations of heat occur in the conductive sublayer which give rise to the formation of a local updraft area. The spacing between the updraft areas is essentially determined by the thickness of the sublayer. The local updraft area develops into a plume or bubblelike structure that draws into it hot fluid from the conductive sublayer. Elsewhere the warm portion of the conductive sublayer becomes thinner, and the removed mass is replenished by cooler fluid from above. Eventually the accumulations of hot air separate from the conductive sublayer and become thermals that gradually rise through the cooler fluid, until they reach the top 
of the layer-which presumably is some sort of solid barrier-where they accumulate. Since on the average the system can lose heat only by conduction through the walls, the accumulation of thermals leads to another conductive layer at the upper surface.

The results of Elder show that the thermal elements eventually become larger until, in equilibrium, they are of the size of the space between the two plates and form a continuous convection region between them. This general picture of convection is essentially independent of the Rayleigh number. At high Rayleigh numbers the fluid between the plates is highly agitated and turbulent, but the convection is nevertheless mainly by thermal plumes of a scale comparable to that of the apparatus. Nonlinear effects do not change much on the horizontal wave numbers associated with the structure of convection, as calculations by Elder (1969) on the basis of Eqs. 3.25 to 3.27 by the method of Herring (1963) have shown. It can consequently be expected that similar behavior might also be found in the free-convection layer of the atmosphere.

\section{FREE CONVECTION CAPPED BY A STABLE LAYER}

There is one essential difference between the convection problem between parallel plates considered up to now and the atmospheric situation. As pointed out in the Introduction, the atmospheric free-convection layer is usually capped by an inversion with a stable temperature gradient. The free-convection layer is highly turbulent, and the stable layer above is quiescent with little turbulence. The development of the convection layer takes place by two processes, the heating of the convection layer and the penetration of the convection layer into the stable region above. This development is particularly well illustrated by Telford and Warner's (1967) airplane measurements of temperature in the atmosphere above uniform terrain in Australia. One of their figures is reproduced as Fig. 3.6. The heat flux from the ground gives rise to a gradual heating of the free-convection layer, whose temperature changes rapidly enough to be essentially uniform (or slightly stable) throughout the free-convection region, above which the stable gradient does not change much. The heat flux is, of course, determined by the gradient across the superadiabatic ground layer, which is not shown in the figure. The convection layer gradually erodes the nonturbulent and stable region above, a process which strongly depends on the mixing processes taking place at the interface.

\section{Growth of the Convection Layer}

A model for predicting the growth of the convection layer must take into account both the processes of heating up of the convection layer and of mixing of the top of the convection layer with the stable flow above. This was clearly recognized by Ball 


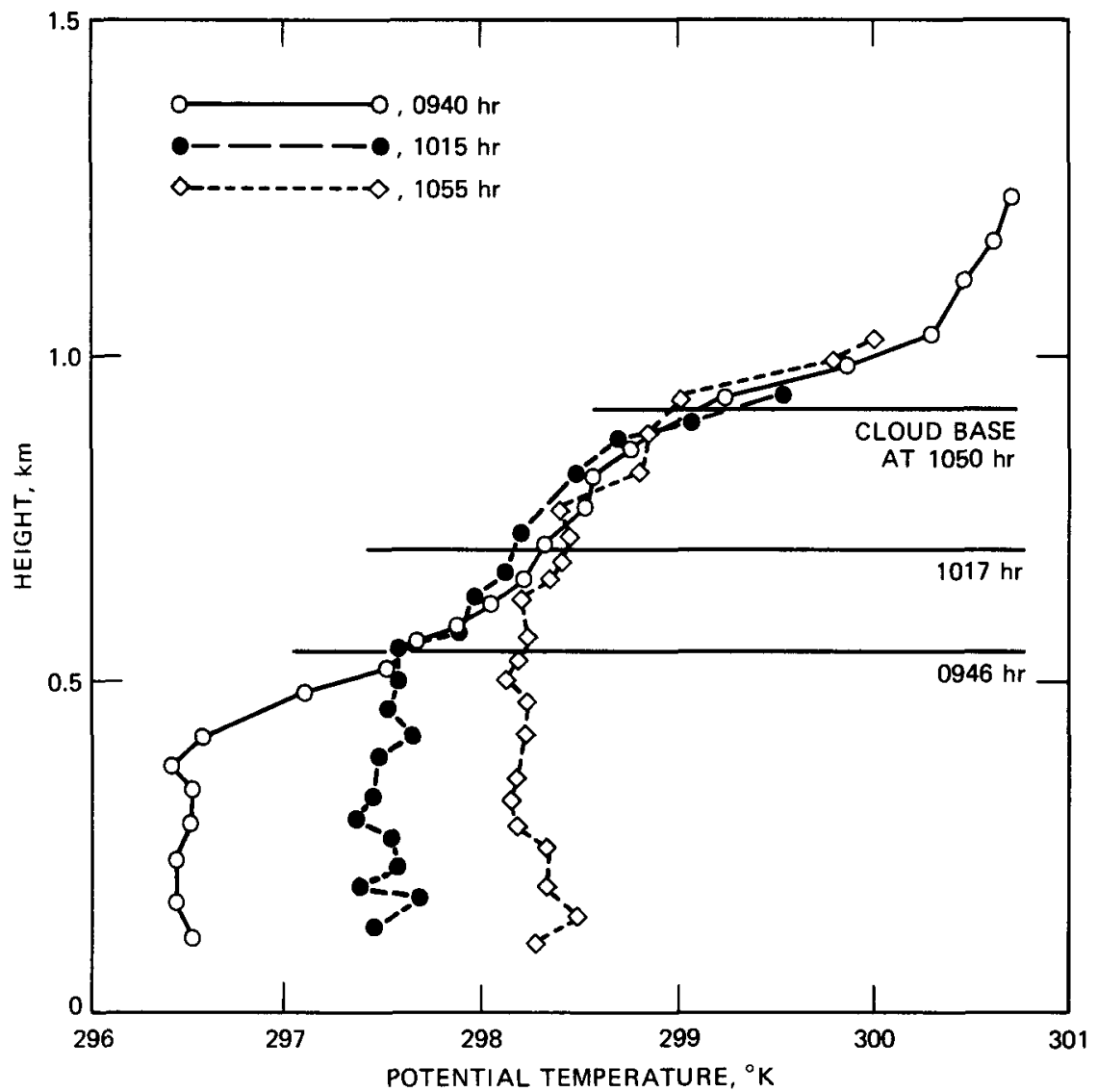

Fig. 3.6 Changes with time of potential temperature. Cloud base was observed during each sounding and in each case can be seen to lie slightly above the height at which there is an abrupt change in potential temperature. [From Wamer and Telford (1967).]

(1960), who developed an analytical model for describing these processes quantitatively. The model has been modified by Lilly (1968), who also considered the effect of radiation from the (cloud-topped) interface between the convection layer and the stable region.

The analysis is greatly simplified if it is assumed that the temperature gradient of the mean temperature field in the convection region is constant and independent of time, so that

$$
\mathrm{T}(\mathrm{t}, \mathrm{z})=\mathrm{T}_{0}(\mathrm{t})+\theta(\mathrm{z})
$$

The conservation-of-heat equation (Eq. 3.20) then becomes 


$$
\frac{d T_{0}}{d t}=\frac{\partial}{\partial z}\left(\bar{k} \frac{\partial \theta}{\partial z}-\langle w T)=-\frac{1}{\rho c_{p}} \frac{\partial H}{\partial z}\right.
$$

where

$$
H=\rho c_{p}\langle w T\rangle-k \frac{\partial \theta}{\partial z}
$$

is the average heat flux at height $z$. In highly turbulent flow the contribution $k(\partial \theta / \partial z)$ is negligible. The average over $w T$ is performed in space over a horizontal plane $\mathrm{z}=$ constant. Integration of Eq. 3.62 yields

$$
H-H_{0}=\left(H_{h}-H_{0}\right) \frac{z}{h}
$$

where the coordinate $\mathrm{z}$ has been measured from the bottom of the convection layer and

$$
\frac{\mathrm{dT}_{0}}{\mathrm{dt}}=-\frac{\mathrm{H}_{\mathrm{h}}-\mathrm{H}_{0}}{\rho \mathrm{c}_{\mathrm{p}} \mathrm{h}}
$$

Note that all the terms in Eq. 3.65 and also $\mathrm{H}$ are time dependent in general, and additional equations are required to obtain quantitative results.

A simple additional equation can be obtained by making an assumption about the joining of the temperature profile at the interface between the stable region and the convection layer. For example, the evidence of Fig. 3.6 suggests an assumption that the temperature in the convection layer is constant, or has a constant slope, throughout the layer while the gradient of the temperature profile in the stable layer also remains unchanged until it intersects the interface. Physically a temperature change of this kind implies that there is no mixing across the interface. Then the difference in the heat flux through the convection layer $\mathrm{H}_{h}-\mathrm{H}_{0}$ arises from two sources, the heating of the convection layer and the cooling of the entrained portion of the stable region into the convection layer, as indicated in Fig. 3.7. If the temperature gradient in the stable-flow region is denoted by $\beta_{0}$, it follows from the geometry of Fig. 3.7 that

$$
\frac{\partial T}{\partial t}=+\beta_{0} \frac{\partial h}{\partial t}
$$

so that, with Eq. 3.65,

$$
-\rho c_{p} \beta_{0} h \frac{\partial h}{\partial t}=H_{h}-H_{0}
$$






Fig. 3.7 Definitions for the inversion-capped convection layer with sharp interface. Linear decrease in heat flux with zero flux at $z=h$.

For a constant heat-flux difference $\mathrm{H}_{\mathrm{h}}-\mathrm{H}_{0}$, over a tıme from $\mathrm{t}=0$ to $\mathrm{t}$, the convection-layer grows in thickness from 0 to $h$, where $h$ is given by

$$
\mathrm{h}^{2}=2 \frac{\mathrm{H}_{0}-\mathrm{H}_{\mathrm{h}}}{\beta_{0} \rho c_{\mathrm{p}}} \mathrm{t}
$$

This result was given by Deardorff, Wills, and Lilly (1969). They also considered constant wall temperature and heat transfer across the conduction layer, as expressed by $\mathrm{Eq} \mathrm{3.45,} \mathrm{where} \mathrm{Nusselt} \mathrm{number} \mathrm{and} \mathrm{Rayleigh} \mathrm{number} \mathrm{are} \mathrm{based} \mathrm{on} \mathrm{the} \mathrm{thickness} \mathrm{of}$ the conduction layer below the convection layer. An explicit solution is no longer possible, but quantitative results are readily obtained.

An interface as envisioned in the derivation of Eq. 3.68 is somewhat of an idealization, even though it seems to be not too far from the real situation during strong inversions, as indicated by such experimental results as those in Fig. 3.6. Equation 3.68 can therefore serve to describe the progression of the interface to a first approximation. However, for a more thorough understanding of the growth of the convection layer, one must consider the details of the mixing process at the interface.

The interface is usually a highly convoluted surface, deformed by thermals or plumes, which carry air from the superadiabatic layer through the convective region. 
These convective elements accelerate as they rise, and, when they arrive at the average interface $(z=h)$ of the convective layer, they have a velocity that exceeds that of the interface and a mean temperature that exceeds that of the surroundings. Therefore they penetrate into the stable region, perhaps even overshooting the level at which their mean temperatures equal that of the surroundings. Some of the kinetic energy of the elements is converted to potential energy of the displaced warmer fluid, but a substantial part becomes turbulent kinetic energy generated as the plume spreads laterally and the now cooler fluid from the plume falls through the warmer fluid to lower zones of equal density. The result of this is that the interface is in constant agitation and strong mixing takes place across it. In fact, the entrainment of air by turbulent mixing from the stable region into the convection layer is the principal mode by which the interface moves upward, and it must be suspected that a layer of substantial thickness exists in which the temperature varies greatly in any horizontal plane from the approximately constant temperature region of the convection layer to the fully developed inversion above.

Ball (1960) and Lilly (1968) have attempted to find a solution to Eq. 3.65 by obtaining a second equation relating $\mathrm{H}_{h}$ and $\mathrm{H}_{0}$ from the turbulent-energy equation (Eq. 2.42 in Chap. 2), which upon integration over the thickness $h$ is:

$$
\begin{aligned}
\int_{0}^{\mathrm{h}} \frac{\mathrm{d}}{\mathrm{dt}} \mathrm{q}^{2} \mathrm{dz}+\overline{\mathrm{w}^{\prime}\left(\frac{\mathrm{p}^{\prime}}{\rho}+\mathrm{q}^{2}\right)_{\mathrm{h}}}=-\int_{0}^{\mathrm{h}} \overline{\mathrm{u}^{\prime} \mathrm{w}^{\prime}} \frac{\partial \overline{\mathrm{u}}}{\partial \mathrm{z}} \mathrm{dz} & \\
& +\int_{0}^{\mathrm{h}} \frac{\mathrm{g}}{\overline{\mathrm{T}}} \overline{\mathrm{w}^{\prime} \theta^{\prime}} \mathrm{dz}-\int_{0}^{\mathrm{h}} \epsilon \mathrm{dz}
\end{aligned}
$$

Both Lilly and Ball neglect the first term on the left. Also, turbulent production is not likely to be strong in the convection region, where shear stresses are low and the velocity distribution varies very little with height. Thus the balance is, with the pressure-fluctuation term also neglected (usually because one does not know what to do with it; measurements of pressure fluctuations are practically nonexistent):*

$$
-\left.\overline{w^{\prime} q^{2}}\right|_{h}=-\frac{g}{T} \int_{0}^{h} \overline{w^{\prime} \theta^{\prime}} d z+\int_{0}^{h} \epsilon d z
$$

Ball neglected the transport term and conjectured that dissipation would be very small, so that

$$
\int_{0}^{h} \overline{w^{\prime} \theta^{\prime}} d z=0
$$

*There is no good reason why the pressure fluctuations are not measured simultaneously with velocity fluctuations in the atmosphere. In the laboratory it is almost impossible to measure the fluctuation term inside the boundary layer because of the large size required for pressure transducers of sufficient sensitivity. 




Fig. 3.8 Definitions for the inversion-capped convection layer with overshoot. Heat flux into layer from above equals heat flux from below, according to model of Ball (1960).

or since the flux (assumed to be positive if entering into the volume from below), according to Eq. 3.64 is linear

$$
\overline{\mathrm{w}^{\prime} \theta_{\mathrm{h}}^{\prime}}=-\overline{\mathrm{w}^{\prime} \theta_{0}^{\prime}}
$$

i.e., the heat flux into the convection layer from below is exactly equal to the heat flux into the top of the convection layer. This means that warm air from above the inversion must be transported downward to heat the convection layer, the top part of the convection layer then cannot be at the temperature of the inversion at height $h$. The temperature in the convection layer must be lower than in the previous case, and a situation such as that shown in Fig. 3.8 results. Again, we can write a simple heat-balance equation for the situation shown, if we assume that the heat flux at the bottom is constant and if we neglect the adjustment in the mean-temperature profile outside the convection layer by postulating a sharp discontinuity in temperatures at the top of the convection layer. The heat flux into the top of the convection layer is equal to the heat flux from below, and the total heat input must be used to heat the 
layer. However, in addition, heat becomes available for heating the layer from the strip of thickness, $\mathrm{dh}$, which is eroded from the stable layer during time $\mathrm{dt}$, and thus the heat-balance equation becomes, with the geometry of Fig. 3.8:

$$
h \frac{d T}{d t}+\left(T_{0}+\beta_{0} h-T\right) \frac{d h}{d t}=\frac{2 H_{0}}{\rho c_{p}}
$$

A second equation relating $\mathrm{h}$ and $\mathrm{T}$ cannot be found as simply as in the previous case. However, it is reasonable to assume that temperature change and layer thickness are proportional, in analogy to Eq. 3.66:

$$
a \frac{\partial \mathrm{h}}{\partial \mathrm{t}}=\frac{\partial \mathrm{T}}{\partial \mathrm{t}} \rightarrow \alpha \mathrm{h}=\mathrm{T}-\mathrm{T}_{0}
$$

where $\alpha$ is an arbitrary constant. With this assumption Eq. 3.73 yields

$$
\mathrm{h}^{2}=4 \frac{\mathrm{H}_{0}}{\beta_{0} \rho c_{\mathrm{p}}} \mathrm{t}
$$

which is identical to Eq. 3.68 , if $\mathrm{H}_{\mathrm{h}}$ is set equal to $-\mathrm{H}_{0}$, as required by the model. The model of Ball leads to precisely the same growth rates as the simple model of Eq. 3.68, and so will any model in which a linear heat-flux distribution is assumed and in which the heat flux at the top lies between zero and $-\mathrm{H}_{0}$. Different assumptions about the dissipation integral in Eq. 3.70 which yield fluxes between 0 and $+\mathrm{H}_{0}$ at the top do not change this result as long as the dissipation is assumed to be constant with height. The growth rate of the layer is thus seen to be independent of the turbulence model.

The assumption of a constant heat flux $\left(\mathrm{H}_{0}\right)$ is, of course, not justified. More elaborate models in which other contributions, such as radiation effects at the top of the layer and a time dependency of the heat flux are considered, have been proposed by Lilly (1968) and Deardorff et al. (1969). The models have to be solved, however, by numerical techniques.

\section{Convection Plumes in the Free-Convection Layer}

A different approach to an understanding of free convection is obtained if the individual elements of the convection-flow process are considered separately. As pointed out earlier, in free convection at small Rayleigh numbers, the convection elements are structures of fairly regular geometry. The flow in the convection layer consists of a vertical region in which heat is moved upward in rather concentrated updraft zones and of other regions in which a downward flow of colder fluid from above takes place at somewhat lower velocities and therefore over a wider area between the updraft regions. The cited laboratory experiments of Elder (1969) 
furthermore have shown that the updraft elements develop from the superadiabatic layer and form concentrated buoyancy elements that determine the structure of the flow and temperature field in the convection layer.

A very similar structure of convective motion has been inferred for the atmosphere also. Bunker (1959) has concluded that thermals, i.e., bubbles of warm air, or hot plumes, rise from the superadiabatic layer into the convection layer where their presence produce upwind regions in which glider planes or birds can soar (Scorer, 1954; Ludlam and Scorer, 1953). The experimental evidence of measurements of fluctuating temperature and velocity with low-flying airplanes suggests that the thermal elements rise with little change in diameter and temperature (for recent evidence, see Warner and Telford, 1967), rather than like a thermal cloud that mixes freely with the surrounding. Scorer and Ludlam (1953) have formulated on this basis a theory of motion of convective thermals, whose behavior is like that of a buoyant bubble traveling at a constant speed equal to the asymptotic rise velocity and whose outer shell is eroded in the process of migration. This erosion takes place partly by convective instabilities and separation of local convective elements from the main cloud and partly by the development of instabilities of the interface between the thermal and its surroundings; these instabilities are induced by the difference in density and velocity (Kelvin Helmholtz instabilities). The bubble model has been developed further by Malkus and Scorer (1955) [see also Roll (1965)].

A different treatment, which is theoretically more appealing and better confirmed by laboratory experiments, considers that the thermal element grows by entrainment of cooler air from the surroundings, a process which reduces effective buoyancy and thus the rate at which the elements rise. The tops of the elements have a spherical appearance, not because of the bubble nature of a blob of hot air rising but because they are circular vortices. This concept has been explored by Turner $(1957,1960)$, who considered the thermal as a buoyant vortex moving in neutral air (Turner, 1957) and stably stratified air (Turner, 1960). On the basis of Turner's work, we briefly outline below the theory of the rise of a convective vortex.

Let the vortex have an initial circulation $\mathrm{K}$, induced by the release of hot air from the superadiabatic layer, and let $R$ be the radius of the vortex. Then its momentum $M$ is, to a first approximation, given by:

$$
\mathrm{M}=\pi \rho \mathrm{KR}^{2}
$$

where $\rho$ is the mean density of the fluid. The change of momentum is caused by the buoyance force, $\rho \mathrm{F}=\mathrm{gV}\left(\rho-\rho^{\prime}\right)$,

$$
\frac{\mathrm{dM}}{\mathrm{dt}}=\rho \mathrm{F}
$$

where $\mathrm{V}$ is the volume of the fluid and $\rho^{\prime}$ is the fluid density in the vortex. In a surrounding with constant density $\rho$, the growth of the initial volume takes place by entrainment of air from the surroundings; consequently the buoyancy of the vortex 
remains constant. A solution of Eqs. 3.76 and 3.77, with the assumption that the uplift velocity $(w$ ) follows a similarity law so that $w \sim K / R$, yields a rise time $t$ between stages where $R=R_{0}$ and $R$, given by:

$$
t=\frac{h^{2}}{4 \pi c^{2} K^{3}} F+\frac{h R_{0}}{c K}
$$

where $h$ is the distance traveled during time $t$ and $c$ is a constant defined by the spread rate

$$
\mathrm{R}=\frac{\mathrm{F}}{2 \pi \mathrm{cK}^{2}} \mathrm{z}
$$

Equations 3.60 and 3.61 require an estimate of $\mathrm{K}$ and $\mathrm{F}$ as well as of the constant $\mathrm{c}$, which cannot be obtained unless something is known about the initial conditions. Nevertheless, it shows that increased buoyancy has the tendency to increase time $t$ and thus to lower the velocity of ascent. A more detailed investigation, by numerical techniques, of the vortex problem has been made by Lilly (1964).

It is often observed that the thermal elements of the convection layer are stationary plumes, of some finite duration, rather than thermals of the kind investigated by Scorer and associates, or Turner. The model of Scorer and Ludlam gives little information on the behavior of thermal plumes, i.e., thermal elements which are steady and which extend throughout the height of the convection layer. Scorer and Ludlam, however, express the notion that the "wake" behind the bubble will be an updraft region into which smaller bubbles, which together form a sort of thermal plume, might be drawn.

An alternate way to treat the thermal elements is by considering them as entities which arise from a local source of buoyancy near the ground and which develop somewhat like jets in free turbulent shear flow. This suggests the use of similarity assumptions for all profiles and suitable models for the shearing stress distribution in the plume on the basis of the methods employed in the treatment of free turbulent shear flow (Schliching, 1968). The earliest treatment along these lines of the thermal plume in a neutral atmosphere was given by Schmidt (1941), who used a mixing-length assumption to close the set of governing equations.

A more extensive treatment, including thermals and thermal plumes rising in a stably stratified layer, has been given by Morton et al. (1956). Later developments of the theory and pertinent experimental results have been summarized in a recent survey paper by Turner (1969) which includes an extensive bibliography.

Briefly, the ideas underlying the aerodynamic model of Morton et al. (1956) for the motion of buoyant elements in a stable surrounding are as follows: They considered the element to be a distinct entity generated by a local source of buoyancy in which both the density defect and the velocity obey similarity distributions. For this plume, they derived a set of conservation equations. To close this set, they did not use the mixing-length model of Schmidt (1941) - which is an assumption on the shear 
stress and heat-flux distribution inside the thermal element-but instead stipulated that the thermal element entrains fluid from the surrounding at a rate proportional to a characteristic velocity of the plume, for which they chose the local velocity averaged across the plume. Finally, they approximated the similarity profiles either by constant values of plume density $\left(\rho^{\prime}\right)$ and velocity (w) over the width (b), which change abruptly for $|x|,|y| \geqslant 1 / 2$ b to $\rho$ and 0 , respectively ("top-hat" distribution), or by the Gaussian-error function, with variance $b^{2}$. Then conservation equations for a steady thermal plume are obtained by integration over the plume at distance $z$ from the virtual heat source, in the form given below.

Change of volume:

$$
\frac{d}{d z}\left(\pi b^{2} w\right)=2 \pi b \alpha w
$$

Conservation of momentum:

$$
\frac{\mathrm{d}}{\mathrm{dz}}\left(\pi \mathrm{b}^{2} \mathrm{w}^{2}\right)=\mathrm{m} \pi \mathrm{cb}^{2} \frac{\rho-\rho^{\prime}}{\rho_{\mathrm{R}}}
$$

Change in density:

$$
\frac{\mathrm{d}}{\mathrm{dz}}\left(\pi \mathrm{b}^{2} w g \frac{\rho-\rho^{\prime}}{\rho_{\mathrm{R}}}\right)=\pi \mathrm{b}^{2} w \frac{\mathrm{g}}{\rho_{\mathrm{R}}} \frac{\mathrm{d} \rho}{\mathrm{dz}}
$$

where the Boussinesq approximations have been used. In these equations, $\rho_{R}$ is a reference density and $\alpha$ is the factor of proportionality between $w$ and the entrainment rate per unit circumference of the plume. The coefficient $m$ is a profile constant, equal to 1 for the top-hat distributions. For a neutrally stratified external flow, Eqs. 3.80 to 3.82 can be integrated readily, leading to a linear spreading of the plume and a temperature (or density) that varies with height as $\mathrm{z}^{-5 / 3}$. These results are formally equivalent to those obtained from the mixing-length theory. Equations 3.80 to 3.83 have the advantage over the mixing-length theory in that no new formulation (for the mixing length) is required in applying them to a stratified atmosphere.

The entrainment coefficient $(\alpha)$ must be determined from experiments to solve the equation, Morton et al. obtained this value by making the assumption that the similarity distributions of density and velocity obeyed a Gaussian or normal distribution, which corresponds to $\mathrm{m}=2$, and by fitting the solution obtained from Eqs. 3.80 to 3.82 for spread rates to experimental data of laboratory studies of plume spread. Data of Rouse et al. (1952) yielded a value of $\alpha \approx 0.08$.

Although the above value of $\alpha$ applies only to plumes rising in neutrally stratified fluids, Morton et al. (1956) applied it also to stable stratification. In that case the plume does not extend from the ground to infinity but it comes to rest when the internal temperature is equal to that in the stable surroundings. The inertia of the plume makes it overshoot this level, and the highest point of the plume is reached 
when the downward buoyancy has reduced the plume velocity to zero The maximum height, $z_{\max }$, at which this occurs has been given by Morton et al as

$$
\mathrm{z}_{\max } \approx 4 \mathrm{~F}_{0}^{3 / 4} \mathrm{~N}^{-3 / 4}
$$

where $F_{0}$ is the total buoyancy flux $\left(1 \mathrm{n} \mathrm{m}^{4} / \mathrm{sec}^{3}\right)$ that enters the plume at ground level and $\mathrm{N}$ is the Brunt-Vaisala frequency, defined by

$$
\mathrm{N}^{2}=\frac{\mathrm{g}}{\mathrm{T}} \beta_{0}
$$

and $\beta_{0}$ is, as before, the temperature gradient of the stable layer

The linear spread rate is a result of the entrainment assumption, and, since a linear growth of the plume is not observed in the atmosphere, many writers feel that this assumption is not realistic Different assumptions have been proposed, for example, Priestley and Ball (1955) suggested to assume that a shear stress exists in the plume whose similarity distribution is scaled by the velocity w squared For a discussion of this model and its comparison with that of Morton et al (1956), reference is made to Priestley (1959) Their model also results in a linear spread rate, and therefore it does not offer much improvement In a recent paper, Telford (1966) attempted to obtain a more realıstic model by postulating an entrainment model based on turbulence both in and outside the plume The plumes obtained with this assumption are more nearly of constant cross section with height, but the mathematics is involved, and results can be obtained only by numerical methods

\section{Structure of the Convection Layer}

In all the plume models, it is assumed that the heat is convected upward by plumes or thermals that are either isothermal bubbles or elements cooled by entrainment of colder air from the surroundings This entrained air also increases the volume of air in the plume The models, however, do not allow for a mechanism by which the air is heated between the plumes. The approximately average temperature in the convection layer indicates that such heating should take place, and qualitatively it is easy to see how this occurs It is evident that a thermal plume not only moves heat but also mass to higher regions This movement must be balanced by a downdraft in other parts of the convection layer In a steady or quasi-steady plume, the mass carried upward is that of the initial mass flux, taken from the superadiabatic layer in the manner described above, and that entrained by the plume on 1ts way upward All of this mass must be released near that height in the inversion layer where the plume velocity is equal to zero Thus the slightly cooler air below the height at which the plume temperature equals that of the surroundings is displaced downward

If the ground temperature increases because of further radioactive heating, it can be expected that the mean temperature of a plume rising out of it also increases The result is that the plume extends to a higher level in the inversion, thereby displacing 
slightly warmer air than before, which moves downward and pushes the previously displaced air ahead of it Consequently the mean temperature between the plumes becomes, in the absence of an effective mixing between the updraft and downdraft regions, slightly stratified Baines and Turner (1969) have suggested that this process is similar to overturning fluid in a box that contains a heat source in the center at the bottom, a case which they studied in the laboratory Although this model is of qualitative interest in that it shows that the process envisioned for heating the spaces between thermal plumes does indeed take place, it is not directly applicable because the dimensions of the box set a second, lateral-length scale to the convection process, whereas, in an atmospheric convection layer, it is likely that the scale is set by the heat flux alone This scale seems to be the same as that for the thermals or plumes because, in their study of the convection layer, Warner and Telford (1967) found that on the average the width between thermals was about equal to the diameter of the thermals

Since the air between the plumes has been part of the stable layer, its turbulent temperature fluctuations must be very small compared to those in the plume This was observed by Priestley (1959), Warner and Telford (1967), and Businger (1969) The variance of the temperature fluctuations averaged over the convection layer is highest near the surface layer and decreases with height Since observations show that the space between the plumes makes practically no contribution to the variance, it follows that the temperature fluctuations decrease with height, which is quite compatible with finding only small temperature fluctuations in the downward moving air Consequently it is possible to find a relation between the average temperature gradient for the whole convective layer from the conservation equation of the turbulent fluctuation balance, Eq 245 of Chap 2 (without radiation term)

$$
\frac{1}{2} \frac{\overline{\partial \theta^{\prime 2}}}{\partial \mathrm{t}}=-\frac{\partial}{\partial \mathrm{z}}-\frac{\overline{\mathrm{w}^{\prime} \theta^{\prime 2}}}{2}-\overline{\mathrm{w}^{\prime} \theta^{\prime}} \frac{\partial \overline{\mathrm{T}}}{\partial \mathrm{z}}-\epsilon_{\mathrm{t}}
$$

The slowness of the heating of the ground makes it likely that the term on the left is small Also, it has been argued by Deardorff (1966), on the basis of measurements of Telford and Warner (1964), that the dissipation term $\epsilon_{\mathrm{t}}$ is negligible However, this can be valid only locally since the production term, averaged over the whole convection layer, must balance the dissipation term When we assume that most of the fluctuations disappear in the process of mixing of the plume mass in the inversion at the top of the convection layer, then $\epsilon_{\mathrm{t}}$ over the bulk of the convection layer above the superadiabatic wall layer can be neglected, the diffusion term thus balances the production term From this result, Deardorff inferred that the heat flux must be against the gradient, and he derived an upper limit to the magnitude of the positive gradient

According to what has been sald, the diffusion term has a value different from zero only in the plume, where the mean updraft velocity is probably substantially larger than the fluctuation velocities, so that $\overline{\mathrm{w}^{\prime} \theta^{\prime 2}} \approx \overline{\mathrm{w}_{\mathrm{p}} \theta^{\prime 2}}$, where $\mathrm{w}_{\mathrm{p}}$ is the average plume velocity Integration of $\mathrm{Eq} 367$ with these assumptions across a horizontal plane $z=$ constant yields 


$$
\mathrm{H} \frac{\partial \overline{\mathrm{T}}}{\partial \mathrm{z}}=-\frac{\partial}{\partial \mathrm{z}} \mathrm{w}_{\mathrm{p}} \overline{\theta^{\prime 2}} \cdot \mathrm{r} \cdot \rho \mathrm{c}_{\mathrm{p}}
$$

where $r$ is the ratio of the plume area to the total area. As long as $r$ is approximately constant and $\mathrm{w}_{\mathrm{p}}$ and $\overline{\theta^{\prime 2}}$ decrease with height in the plume, the term on the right-hand side of Eq. 3.86 is positive, i.e., a positive heat flux causes a positive temperature gradient, contrary to the usual conduction situation. Note, however, that some drastic assumptions are required to arrive at this result, which must ultimately be justified by recourse to experiment. A similar line of reasoning as that given here was followed by Businger (1969).

\section{EXPERIMENTS ON THE FREE-CONVECTION LAYER}

Since there exists a tremendous amount of literature on free convection, it is the opinion of this writer that at present it is not necessary for an understanding of the atmospheric convection layer to perform any further experiments on steady free convection at a small scale. However, it appears necessary that the abundance of theoretical studies be supplemented by a few critical experiments to validate conjectures or hypotheses on which the theoretical or numerical models are based. Only in this manner can fruitful courses for further theoretical research be discovered.

Unsteady convection, on the other hand, appears to be very similar in laboratory and field, at least in its average features. It is unclear how far the parallel goes, and there is very little knowledge of the structure of the convection elements in the laboratory except for the qualitative description of Elder (1969) and Foster (1965). It also seems desirable to study the structure of the thermals or plumes and the mechanism of depletion of, and the accumulation of hot air from, the superadiabatic layer. What would be the influence on these processes of a weak shear flow?

But the most promising and perhaps most important area of research lies in measurements of the process of reverse development of a convection layer. When the heat flux from the wall becomes zero, then the roughly constant temperature of the convection layer lets it appear as a layer of decaying temperature and velocity fluctuations. How does this process of decay take place? Is it gradual, or does the flow field break down suddenly? How does the inversion migrate downward? How is this process affected by the downward flux of heat during radiation cooling of the ground? These questions are of great importance for our understanding of the atmospheric boundary layer.

\section{REFERENCES}

Baines, W. D., and J. S. Turner, 1969, Turbulent Buoyant Convection from a Source in a Confined Region, J. Fluid Mech., 37: 51-80. 
Ball, F. K., 1960, Control of Inversion Height by Surface Heating, Quart. J. Roy. Meteorol. Soc., 86: 483-494.

Bunker, A. F., 1959, Marine Meteorology; Cloud and Environment Velocities, Temperatures, Humidities and Water Contents Associated with a Shear Zone in the Trade Winds, Woods Hole Oceanographic Institution, Technical Report 44.

Businger, J. A., 1969, On the Structure of the Unstable Atmospheric Boundary Layer, Radio Sci., Dec.

Busse, F. H., 1969, On Howard's Upper Bound for Heat Transport by Turbulent Convection, $J$. Fluid Mech., 37: 457-477.

Catton, I., 1966, Natural Convection in Horizontal Liquid Layers, Phys. Fluids, 9: 2521-2522.

Chandrasekhar, S., 1961, Hydrodynamic and Hydromagnetic Stability, Clarendon Press, Oxford.

Currie, I. G., 1967, The Effect of Heating Rate on the Stability of Stationary Fluids, J. Fluid Mech., 29: 337-347.

Deardorff, J. W., 1966, The Counter Gradient Heat Flux in the Lower Atmosphere and in the Laboratory, J. Atmos. Sci., 23: 503-506.

- and G. E. Willis, 1967a, Investigations of Turbulent Convection Between Horizontal Plates, $J$. Fluid Mech., 28: 675-704. and G. E. Willis, 1967b, The Free Convection Temperature Profile, Quart. J. Roy. Meteorol. Soc., 93: 166-175.

_, G. E. Willis, and D. K. Lilly, 1969, Laboratory Investigation of Non-Steady Penetrative Convection, J. Fluid Mech., 35: 7-31.

Elder, J. W., 1968, The Unstable Interface, J. Fluid Mech., 33: 69-96. 1969, The Temporal Development of a Model of High Rayleigh Number Convection, J. Fluid Mech., 35: 417-437.

Foster, T. D., 1965a, Stability of a Homogeneous Fluid Cooled Uniformly from Above, Phys. Fluids, 8: 1249-1254.

—, 1965b, Onset of Convection in a Layer of Fluid Cooled from Above, Phys. Fluids, 8: $1770-1774$.

Globe, S., and D. Dropkin, 1959, Natural Convection Heat Transfer in Liquids Confined by Two Horizontal Plates and Heated from Below, J. Heat Transfer, 81: 24-28.

Goldstein, R. J., and D. J. Graham, 1969, Stability of a Horizontal Fluid Layer with Zero Shear Boundaries, Phys. Fluids, 12: 1133-1137.

Herring, J. R., 1963, Investigation of Problems in Thermal Convection, J. Atmos. Sci,, 20: 325-338.

- 1964, Investigation of Problems in Thermal Convection: Rigid Boundaries, J. Atmos. Sci., 21: 277-290.

_., 1966, Some Analytical Results in the Theory of Thermal Convection, J. Atmos. Sci., 23: $672-677$.

_, 1969, Statistical Theory of Thermal Convection at Large Prandtl Numbers, Phys. Fluids, 12: 39-52.

Howard, L. N., 1963, Heat Transport by Turbulent Convection, J. Fluid Mech., 17: 405-432.

Kraichnan, R. H., 1962, Turbulent Thermal Convection at Arbitrary Prandtl Numbers, Phys. Fluids, 5: 1374-1389.

Lick, W., 1965, The Instability of a Fluid Layer with Time Dependent Heating, J. Fluid Mech., 21: 565-576.

Lilly, D. K., 1964, Numerical Solutions for the Shape Preserving, Two Dimensional Thermal Convection Element, J. Atmos. Sci., 21: 83-98.

—, 1968, Models of Cloud-Topped Mixed Layers Under Strong Inversions, Quart. J. Roy. Meteorol. Soc., 94: 292-309.

Ludlam, F. H., and R. S. Scorer, 1953, Convection in the Atmosphere, Quart. J. Roy. Meteorol. Soc., 79: 317-341.

Malkus, J. S., and R. S. Scorer, 1955, The Erosion of Cumulus Towers, J. Meteorol., 12: 43-47. 
Malkus, W. V. R., 1954a, The Heat Transport and Spectrum of Thermal Turbulence, Proc. Roy. Soc. (London), Ser. A, 225: 195-212.

_, 1954b, Discrete Transitions in Turbulent Convection, Proc. Roy. Soc. (London), Ser. A, 225: 195-212.

_, and G. Veronis, 1958, Finite Amplitude Cellular Convection, J. Flutd Mech., 4: 225-260.

Morton, B. R., G I. Taylor, and J. S. Turner, 1956, Turbulent Gravitational Convection from Maintained and Instantaneous Sources, Proc Roy Soc (London), Ser, A, 234: 1-23

Pellew, A., and R. V. Southwell, 1940, On Mantained Convective Motion in a Fluid Heated from Below, Proc. Roy Soc (London), Ser A, 176: 312-343.

Prandtl, L., 1932, Meteorologıshe Anwendungen der Strömungslehre, Bettr. Phys. Atmos., 19: 188-202.

Priestley, C. H. B., and F. K. Ball, 1955, Continuous Convection from an Isolated Source of Heat, Quart J. Roy Meteorol Soc., 81: 144-157.

, 1959, Turbulent Transfer in the Lower Atmosphere, The University of Chicago Press, Chicago.

Rayleigh, Lord, 1961, On Convective Currents in a Horizontal Layer of Fluid When the Higher Temperature Is on the Under Side, Phll. Mag., 32: 529-546.

Robinson, J., 1967, A Note on the Stabilty of an Infinite Fluid Heated from Below, $J$ Flutd Mech., 29: 461-464.

Roll, H U., 1965, Phystcs of the Marine Atmosphere, Academic Press Inc., New York.

Rouse, H., C. S. Yih, and H. W. Humphrey, 1952, Gravitational Convection from a Boundary Source, Tellus, 4: 201-210.

Schlichting, H., 1968, Boundary Layer Theory, 6th ed., McGraw-Hill Book Company, Inc., New York.

Schmidt, W., 1941, Turbulente Asbre1tung eınes Stromes Erhitzter Luft, Z. Angew, Math Mech., 21: 255-278.

Scorer, R. S., and F. H. Ludlam, 1953, Bubble Theory of Penetrative Convection, Quart. J. Roy, Meteorol Soc., 79: 94-103.

, 1954, The Nature of Convection as Revealed by Soaring Burds and Dragon Flies, Quart. J. Roy. Meteorol. Soc, 80: 68-77.

Silveston, P. L., 1958, Wärmedurchgang in waagerechten Flüssigkeltsschichten, Forsch Ingenteurw., 24: 29-32 and 59-69.

Spangenberg, W. G., and W. R. Rowland, 1961, Convective Circulation in Water Induced by Evaporative Cooling, Phys. Fluids, 4: 743

Spiegel, E. A., 1962, On the Malkus Theory of Turbulence, in The Mechanics of Turbulence, Gordon and Breach, Science Publishers, Inc., New York, pp. 181-195.

Telford, J. W., and J. Warner, 1964, Fluxes of Heat and Vapor in the Lower Atmosphere Denved from Aircraft Observations, J. Atmos. Sct., 21 : 539-548.

Thomas, D. B, and A. A. Townsend, 1958, Turbulent Convection over a Heated Horizontal Surface, J. Fluld Mech , 2: 473-492.

Townsend, A. A., 1962, Remarks on the Malkus Theory of Turbulent Flow, in The Mechanics of Turbulence, Gordon and Breach Science Publishers, Inc., New York.

Turner, J. S., 1957, Buoyant Vortex Rings, Proc. Roy Soc ,(London), Ser A, 239: 61-75. 1960, A Comparison Between Buoyant Vortex Rings and Vortex Parrs, J. Flutd Mech., 7: 419-432.

1969, Buoyant Plumes and Thermals, in Annual Review of Flutd Mechanics, Vol 1, Annual Reviews Inc, Palo Alto, pp 29-44

Warner, J., and J. W. Telford, 1967, Convection Below Cloud Base, J. Atmos. Sct., 24: 374-382.

Wesseling, P., 1969, Laminar Convection Cells at High Rayleigh Numbers, J. Flutd Mech., 36: 625-637.

Wills, G. E., and J. W. Deardorff, 1967, Confurmation and Renumbering of the Discrete Heat Flux Transitions of Malkus, Phys. Fluids, 10: 1861-1866. 
-

○ 


\section{TWO-DIMENSIONAL DISTURBED \\ BOUNDARY LAYERS}

\section{INTRODUCTION}

The assumption of uniformly rough terrain at uniform temperature and of unlimited extent is an idealization to which natural terrain corresponds only in exceptional cases In fact, field experiments for verifying the profile laws described in previous chapters always had to be conducted at carefully selected sites that were located only after extensive searches. This was necessary because of the boundary-layer nature of the atmospheric surface layer. Turbulent boundary layers have a "long memory," 1.e., disturbances of their equilibrium persist over long distances, and the fluid particles in them have a high propagation velocity along a surface as compared with that in the direction normal to 1 . Consequently disturbances might have been generated at the surface at large distances upstream from the point at which measurements are taken, but because they damp out only slowly they are still reflected in the velocities at a comparatively short distance from the ground. Boundary layers that show the influence of boundary configurations different from the local ones will be termed "disturbed" boundary layers.

Systematic studies of disturbed boundary layers began rather recently. The greatest progress has since been made on the problem of the two-dimensional internal boundary layer. This is the boundary layer which develops inside another boundary layer when the flow that has approached over uniform terrain encounters a step 
change in surface conditions, such as a change in terrain from smooth to rough or a change of surface temperatures from hot to cold, or vice versa, and when the discontinuity extends normal to the direction of flow For this type of problem, boundary-layer analysis can be applied except directly at the discontinuity

Meteorologists became interested in internal boundary layers after finding that some of the sites selected for micrometeorological field experiments yielded flow characteristics significantly different from those to be expected over truly uniform terrain By assuming that the spatial acceleration or advection terms of the boundary layer were not equal to zero, they could explain some of the differences Today the internal boundary layers can be considered in the planning of micrometeorological field experiments The theory is sufficiently developed to determine the necessary fetch length for a surface to have adjusted over sufficient height to the new surface conditions The criterion for this adjustment to have taken place is that the ground shear stress has become constant along the ground and that the constant shear layer corresponding to this stress extends over the whole height of interest Apart from this application, internal boundary layers should be useful eventually in the quantitative prediction of evaporation from small lakes, or from irrigated fields in dry land, and in other problems of similar nature where the distance is short over which the wind encounters changed boundary conditions The first part of this chapter will be a review of internal boundary-layer theory

The second problem, to be treated only qualitatively in this chapter, is the air flow near shelterbelts Shelterbelts have been used from time immemorial, but fluidmechanics studies of the flow field, with the aim of deriving design criteria for a most efficient shelterbelt, are of very recent origin and have as yet not led to methods of predicting the flow field downstream and ahead of shelterbelts Nevertheless, a number of facts are known about shelterbelt effects, and their fluid-mechanics aspects will be reviewed here

\section{THE INTERNAL BOUNDARY LAYER}

A typical situation of the boundary-layer flow over a sudden change in roughness from a surface whose roughness is described by the roughness height $z_{01}$ to a rougher surface w1th roughness height $z_{02}$ is shown in $F_{1} g \quad 41$ Here we assume that the roughness heights on either side of the discontinuity correspond to terrain of infinite extent There is no fluid-mechanics principle that requires the roughness height to be constant in such a situation, nevertheless, all the models of internal boundary layers that have been advanced as yet require that the values of $z_{0}$ be known upstream and downstream from the discontinuity in roughness Usually the assumption is made that the $z_{0}$ values are dependent on the geometries and arrangements of the elements of surface roughness only and that they correspond to values found from wind-profile measurements over uniform terrain of the same configuration as that existing locally in complex terrain 




Fig. 4.1 Schematic representation of the development of an internal boundary layer flow from smooth to rough

The boundary-layer nature of the flow field upstream and downstream of the roughness discontinuity makes it unlikely that the velocity field at a large distance upstream of the discontinuity and at a large height above the downstream rough surface is affected by the change in roughness Therefore a region of influence of the downwind roughness can be identified by the curve shown in Fig 41 and is called the internal boundary layer of thickness $\delta$ Above the $\delta$ height lies the outer layer where the flow field still is characteristic of the approach conditions, except for a displacement $\delta^{\prime}$ of the outer flow field that is required by the condition of continuity. Very near the ground, on the other hand, an inner layer exists where the proftle has a shape that is fully governed by the local boundary conditions, and in it the existence of an outer flow is reflected only in the velocity scale of the profile Between the inner and the outer layers there exists a blending layer in which the velocity distribution gradually changes from the logarithmic profile above the downstream roughness to that over the upstream one At large distances from either side of the discontinuity in roughness, the shear stress of the surface is adjusted again to such values as would be expected if the flow field had developed over uniform terrain

\section{The Experimental Evidence}

That the qualitative description given in the preceding paragraphs correctly represents the internal boundary-layer situation is borne out by experimental observation Jacobs (1939), who followed a suggestion of Prandtl, made the first experimental investigations of the internal boundary-layer problem The Jacobs experıments were performed in duct flow Simılar experıments in pipe flow by Logan 
and Jones (1963) and in ducts by Tanı and Makita (1968) and Antonia and Luxton (1968) provided experımental data that were not directly applicable to atmospheric conditions, which in view of the discussions in Chap 1 require a very small pressure gradient Atmospheric data from Stearns (1964) and Stearns and Lettau (1963) were based on experiments over the ice of Lake Mendota, W1s, with artificial downwind roughness consisting of bushel baskets or dried pine trees These roughness elements were rather large, and the number of profiles taken was not sufficient to provide a sensitive test to check theoretical models The best experimental data now avalable on mean velocity profiles were obtained by Bradley (1968) who measured simultaneously velocity profiles and ground shear stresses The results of his experiments are shown in Fig 42 , both for the flow from a smooth to a rough surface (Fig $42 a$ ) and for the flow from a rough to a smooth surface $\left(\mathrm{F}_{1} \mathrm{~g} 4 \mathrm{2b}\right.$ ) The velocities are made nondimensional with suitable reference velocities These are velocities which are measured at that height, in centimeters, which is indicated by the index The solid lines and the data points represent measurements Note that the uppermost solid line in $F_{1}$ g $4 \mathrm{~b}$ and the lowest solid line in $F_{1 g} 42 \mathrm{a}$ correspond to the velocity distributions upstream of the discontinuity in roughness, which are seen to be well represented by logarithmic profiles

Several facts stand out clearly from Bradley's experiment First, the mean velocity profile is practically unchanged above that point where the lower part of the profile intersects the original upstream profile This implies that the deflection of the streamlines in the change-of-roughness problem of Bradley is very small, and so $\delta^{\prime}$ is a small quantity This is due partly to the relatively small change in roughness used by Bradley, it is believed that $\delta^{\prime}$ is of significantly larger magnitude if the flow is, for example, from a snow-covered plain onto a forest, from a shore onto a lake, or from ice onto bushel baskets Comparable experimental results with similarly detailed measurements on such situations are, however, currently not avallable from field observations Similar results have been obtained in the laboratory, such as the flow field over multiple fences studied by Kung (1970) or the flow from a smooth boundary onto model trees (Meroney, 1968)

Second, we observe that there is only a small portion of the profile in which the velocity distribution deviates from either the logarithmic profile near the ground or aloft Both these observations and the smallness of $\delta^{\prime}$ have been employed successfully in simplifying the analytical treatment of the problem

The shear stresses at ground level as measured by Bradley are shown below the velocity profiles in Fig 42 The local shear stresses were made nondimensional by dividing by the uniform upstream values and were plotted aganst the distance $x$ from the discontinuity Notice that in the smooth-to-rough problem the shear stress initially rises very sharply to about twice its value at large fetches, whereas in the rough-to-smooth case a drop to about half the large fetch value occurs Theories must account for these features 


\section{The Analytical Formulation of the Internal Boundary-Layer Problem}

The basic equations for a two-dimensional, incompressible turbulent flow in neutral stratıfication are, under steady conditions and averaged over time,

$$
\begin{aligned}
& \overline{\mathrm{u}} \frac{\partial \overline{\mathrm{u}}}{\partial \mathrm{x}}+\overline{\mathrm{w}} \frac{\partial \overline{\mathrm{u}}}{\partial \mathrm{z}}=-\frac{\partial}{\partial \mathrm{x}}\left(\frac{\overline{\mathrm{p}}}{\rho}+\overline{\mathrm{u}^{\prime 2}}\right)-\frac{\partial}{\partial \mathrm{z}} \overline{\mathrm{u}^{\prime} \mathrm{w}^{\prime}} \\
& \overline{\mathrm{u}} \frac{\partial \overline{\mathrm{w}}}{\partial \mathrm{x}}+\overline{\mathrm{w}} \frac{\partial \overline{\mathrm{w}}}{\partial \mathrm{z}}=-\frac{\partial}{\partial \mathrm{z}}\left(\overline{\overline{\mathrm{p}}}+\overline{\mathrm{w}^{\prime 2}}\right)-\frac{\partial}{\partial \mathrm{x}} \overline{\mathrm{u}^{\prime} \mathrm{w}^{\prime}}
\end{aligned}
$$

in which only the viscous terms have been neglected The only additional available equation is the equation of continuity,

$$
\frac{\partial \bar{u}}{\partial \mathrm{x}}+\frac{\partial \overline{\mathrm{w}}}{\partial \mathrm{z}}=0
$$

Thus we have a system of three equations with six unknowns which has to be solved.

In all published models of disturbed boundary layers, the terms with $\overline{u^{\prime 2}}$ and $\overline{w^{\prime 2}}$ have been neglected at the outset Although observations show that there are substantial variations of these quantities from point to point, the contributions made by these to the momentum balance are always found to be small compared to those of the other terms An assumption that may improve on the simple omission of the normal turbulent stress would be that of local isotropy, t.e., $\overline{\mathrm{u}^{\prime 2}}=\overline{\mathrm{w}^{\prime 2}}$, in which case a modified pressure $\tilde{p}=\bar{p}+\rho \overline{u^{\prime 2}}$ may be used. Note that at large distances from the discontinuity in boundary conditions both upstream and downstream, the flow along the ground is of boundary-layer nature, so that the second equation reduces to

$$
\overline{\mathrm{p}}+\rho \overline{\mathrm{w}^{\prime 2}}=\mathrm{p}_{\mathrm{w}}
$$

where $p_{w}$ is the static pressure at the ground, and the pressure-gradient term in the first equation becomes

$$
\frac{\partial}{\partial \mathrm{x}}\left(\frac{\overline{\mathrm{p}}}{\rho}+\overline{\mathrm{u}^{\prime 2}}\right)=\frac{1}{\rho} \frac{\mathrm{dp}}{\mathrm{dx}}+\frac{\partial}{\partial \mathrm{x}}\left(\overline{\mathrm{u}^{\prime 2}}-\overline{\mathrm{w}^{\prime 2}}\right)
$$

In flows that are of boundary-layer nature, the changes of $\overline{u^{12}}-\overline{w^{12}}$ with $x$ are very small, and the second term on the right can safely be neglected. Therefore the pressure gradient is impressed by conditions outside the boundary layer which determine $p_{w}$.

Near the discontinuity in surface conditions, the vertical velocity changes rapidly with distance $x$, and, strictly speaking, this change cannot be neglected. The change 

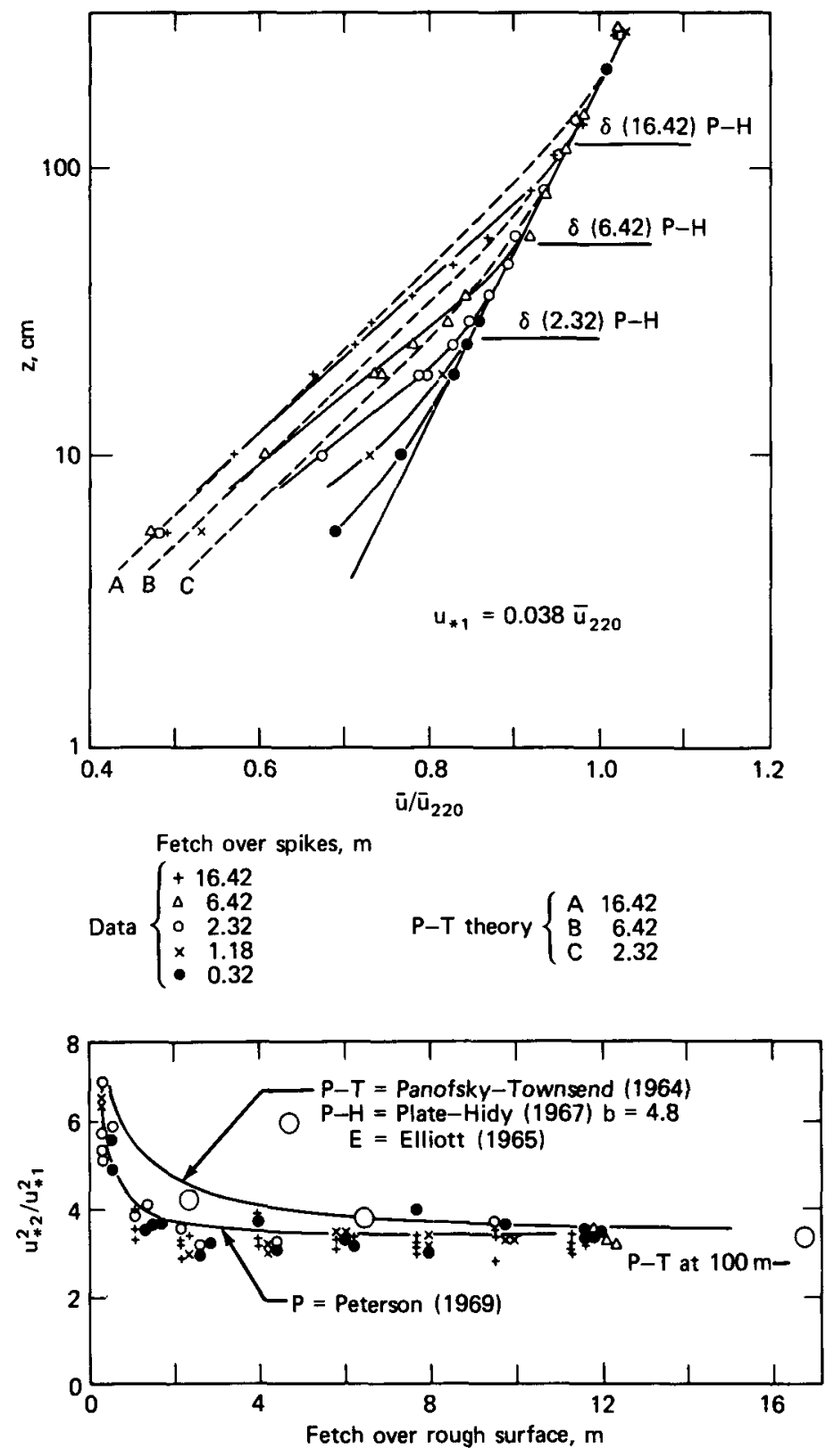

(a) Variation of surface shearing stress downwind of smooth-rough transition $\left(z_{0}=0.002 \mathrm{~cm}\right.$ to $\left.z_{0}=0.25 \mathrm{~cm}\right)$.

(From Bradley 1968) 


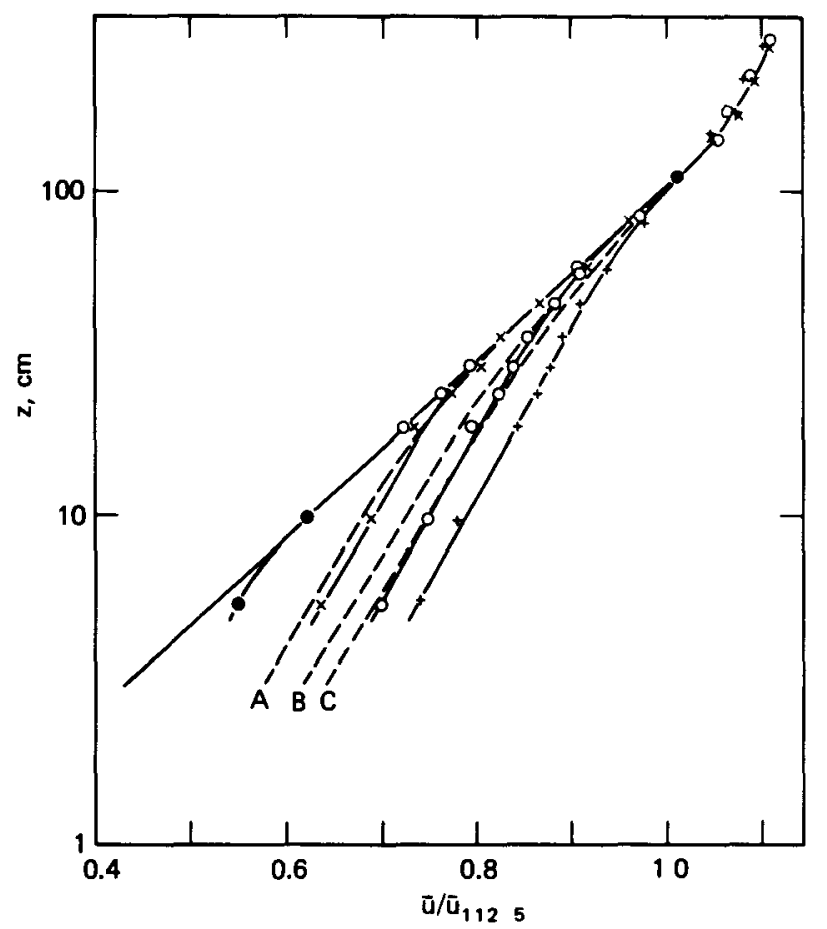

Fetch over tarmac, $m$

Data $\left\{\begin{array}{rr}+1220 \\ 0 & 610 \\ x & 2.10 \\ \bullet & 012\end{array} \quad\right.$ P-T theory $\left\{\begin{array}{lr}A & 2.10 \\ B & 6.10 \\ C & 1220\end{array}\right.$

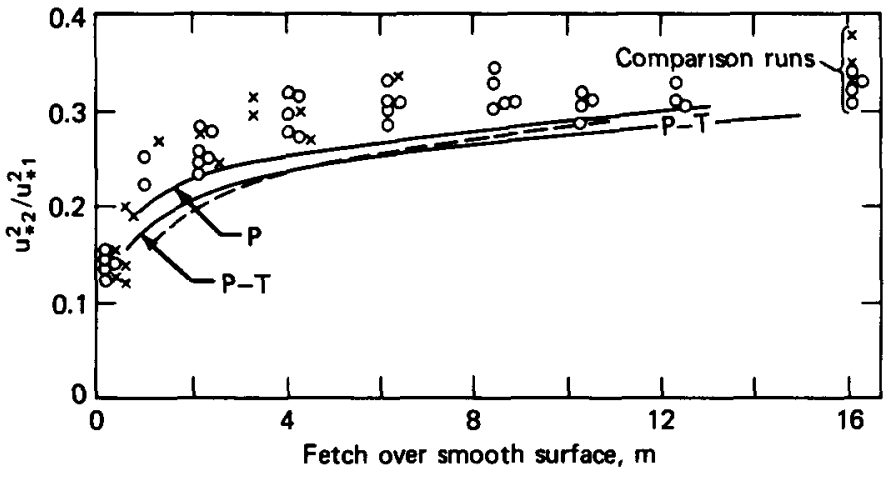

(b) Varıation of surface shearing stress downwind of rough-smooth transition $\left(z_{0}=0.25 \mathrm{~cm}\right.$ to $\left.z_{0}=0.002 \mathrm{~cm}\right)$.

(From Bradley, 1968)

Fig. 4.2 The experımental data of Bradley (1968), compared with results from different models 
gives rise to local pressure gradients both in the $\mathrm{z}$ and $\mathrm{x}$ directions which strongly affect the velocity field These local pressure gradients are responsible for many local distortions in the velocity field One example is the often observed feature that near the edge of a tree stand or a field ( $1 \mathrm{e}$, in canopy flows) the velocities near the ground are much higher than those further inside the canopy. This effect is particularly evident in some experimental results of Meroney (1968), an example of which is shown in Fig 4.3. These data also show that for small $x$ and in the canopy the turbulent intensities $\left(\overline{\mathrm{u}^{\prime 2}} / \overline{\mathrm{u}^{2}}\right)^{1 / 2}$ are overshooting those of large $\mathrm{x}$ values We infer that the region near the discontinuity is one of intense turbulence production which creates a strong stress field, so that adjustments to approximately similar profiles take place over a comparatively short distance $\mathrm{x}$

We note also that the strong pressure field which develops near the roughness discontinuity leads to an initial deflection of the streamlines away from the rough surface. Thus, as is seen in the drag measurements on individual trees shown at the bottom of Fig 4.3, only the first row of trees suffers a greatly increased drag, whereas the succeeding rows of trees are actually subjected to less drag than those at large fetches In extreme conditions, when the first row of trees is so dense that it acts aerodynamically similar to a solid wall, it may even happen that the air flow separates from the front row, subjecting the next few tree rows to a flow in a separation region which exerts a drag in the direction opposite to the flow direction However, such occurrences, which are of ten observed in the related case of the "backward facing step," have not been reported for natural surfaces

There exists only one set of experimental data in which the pressure distributions near the discontinuity have been measured in addition to the velocity field. These data were taken by Yeh (oral communication). The pressure change was considered in numerical calculations by Wagner (1966) and by Onish1 and Estoque (1968) who found that in the simple case of roughness changes the pressure acts as a smoothing function that makes the change from the upstream to the downstream profile across the discontinuity less abrupt However, both the experiments of Yeh and the numerical results show that the pressure effect is restricted to a farly narrow region in the neighborhood of the discontinuity The pressure effect on the downstream boundary layer is very likely a small shift of the origin of the internal boundary layer which appears to begin not at the roughness discontinuity but at a location upstream or downstream from it, much as in the manner that a virtual origin appears in the solutions of other boundary-layer problems in which the boundary-layer assumptions are not satisfied at the physical origin of the boundary layer, such as turbulent jets and wakes.

In the following text we shall not only make the assumption that the pressure is constant across the layer but also that it is constant everywhere Modifications required to account for a pressure gradient in the direction of flow are fairly simple and can readily be incorporated into the models This fact has been demonstrated by Plate and Hidy (1967). With the assumption of $\mathrm{p}_{\mathrm{w}}=$ constant, the set of equations to be solved for the internal boundary-layer problem is reduced to 


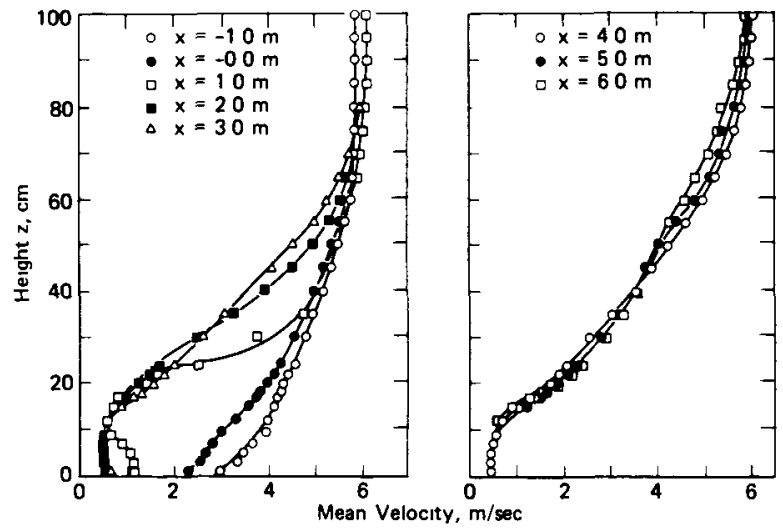

(a) Mean velocity distributions
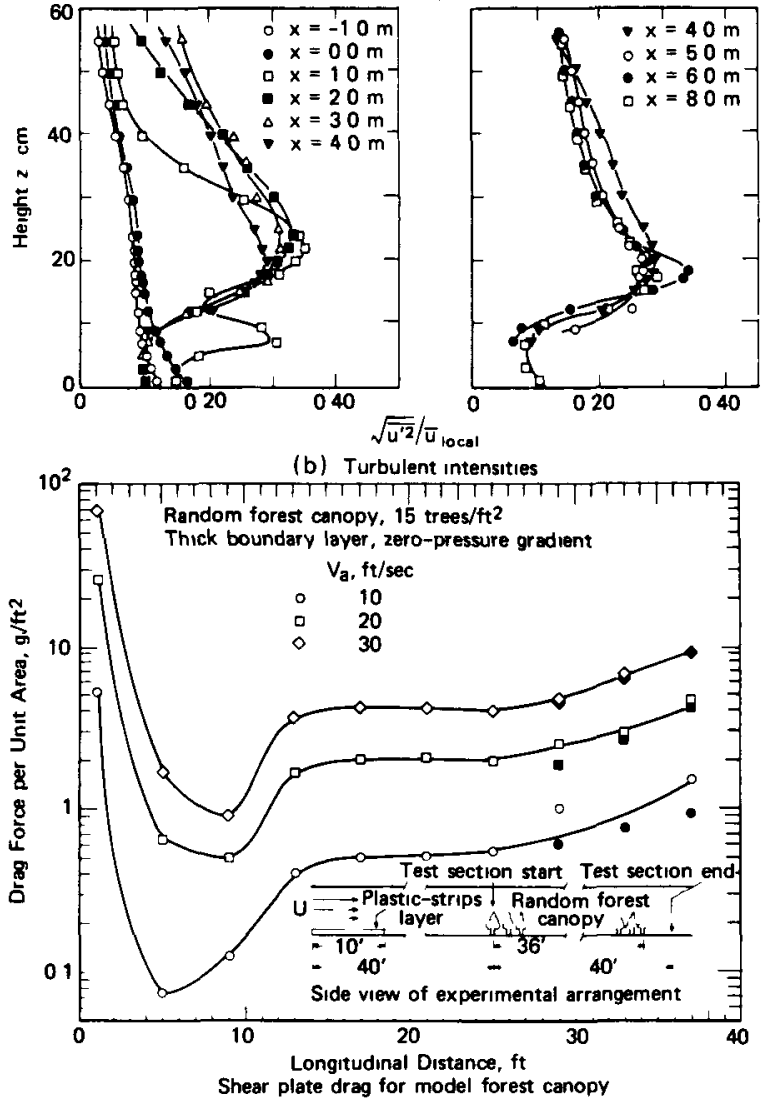

(c) Drag

Fig. 4.3 Velocity distributions, turbulent intensities, and surface drag of a model forest (from Meroney, 1968) 


$$
\begin{gathered}
\overline{\mathrm{u}} \frac{\partial \overline{\mathrm{u}}}{\partial \mathrm{x}}+\overline{\mathrm{w}} \frac{\partial \overline{\mathrm{u}}}{\partial \mathrm{z}}=\frac{\partial \tau}{\partial z} \\
\frac{\partial \overline{\mathrm{u}}}{\partial \mathrm{x}}+\frac{\partial \overline{\mathrm{w}}}{\partial \mathrm{z}}=0
\end{gathered}
$$

To these equations we must add a constituent equation for (the kinematic shear stress) $\tau$ and boundary conditions Both conditions are intımately related to the method of solution chosen.

Apart from an early method, in which the internal boundary-layer problem is treated as a diffusion problem (Philıp, 1959, Dyer, 1963), there are two main methods for solving Eqs. 46 and 4.7. In one of the two methods, solving is by numerical techniques and, in the other, by approximate techniques based on the method of Karman-Pohlhausen (Schlichtıng, 1968, p. 192). The latter, which was introduced to the subject of internal boundary-layer calculations by Elliott (1958), avolds the problem of specifying shear-stress distributions and thus eliminates the need for much of the guessing about the relation between shear stress and mean velocity distribution The guessing is replaced by much safer and more easily experimentally demonstrated assumptions on the mean velocity distribution

\section{Karman-Pohlhausen Method for Predicting the Characteristics of the Internal Boundary Layer}

The Karman-Pohlhausen method is an approximate method for calculating boundary-layer parameters on the basis of an assumed velocity profile in the boundary layer The velocity profile is so chosen that it satisfies a number of boundary conditions at the ground and at the edge of the boundary layer, as well as some integral constraints that are imposed on the flow field by the equations of motion and continuity and by the configuration of the surface. The important integral constraint imposed by the Karman-Pohlhausen method is the integral momentum equation, which is obtained by a formal integration of Eq. 4.6 across the internal boundary layer. This is easily accomplished and leads to the equation

$$
\frac{\mathrm{d}}{\mathrm{dx}} \int_{0}^{\delta} \overline{\mathrm{u}}^{2} \mathrm{dz}-\overline{\mathrm{u}}_{\delta} \frac{\mathrm{d}}{\mathrm{dx}} \int_{0}^{\delta} \overline{\mathrm{u}} \mathrm{dz}=\tau_{\delta}-\mathrm{u}_{* 2}^{2}
$$

where the subscript $\delta$ denotes conditions at the edge $\delta$ of the internal boundary layer and $u_{* 2}^{2}$ is the kinematic shear stress at the ground at $x$.

In view of the physical situation described in the section entitled The Analytical Formulation of the Internal Boundary-Layer Problem, the conditions at $\delta$ are determined by the upstream flow, so that, with reference to Fig. 4.1,

$$
\overline{\mathrm{u}}_{\delta}=\overline{\mathrm{u}}_{1} \text { at } \mathrm{z}=\delta-\delta^{\prime}
$$


and, since the boundary layer upstream of the discontinuity is assumed to be a constant-stress layer over the height considered, it follows also that

$$
\tau_{\delta}=\mathrm{u}_{* 1}^{2}
$$

To complete the system of equations, we must specify the velocity distribution $\overline{\mathrm{u}}_{2}$ that not only determines the integrals in Eq. 4.8 but also the displacement $\delta^{\prime}$. A useful profile is

$$
\frac{\overline{\mathrm{u}}_{2}}{\mathrm{u}_{* 2}}=\frac{1}{\kappa} \ln \frac{\mathrm{z}}{\mathrm{z}_{02}}+f\left(\frac{\mathrm{z}}{\delta}\right)
$$

Since the velocity distribution must become logarithmic near the ground, the function $\mathrm{f}(\mathrm{z} / \delta)$ must be zero for $\mathrm{z} \rightarrow 0$. Furthermore, continuity of the velocity distribution requires

$$
\frac{\overline{\mathrm{u}}_{\delta}}{\mathrm{u}_{* 2}}=\frac{1}{\kappa} \ln \frac{\delta}{\mathrm{z}_{02}}+\mathrm{f}(1)
$$

Elliott (1958) in his pioneering paper on the subject made the assumptions that $\mathrm{f}=0$ and $\delta^{\prime}=0$ so that Eqs. 4.8 to 4.12 lead to a set of two equations for the two unknowns, $u_{* 2}$ and $\delta$. The integration of Eq. 4.8 yields a rather complicated algebraic expression. Elliott was able to show, however, that except near $\mathrm{x}=0$ (where, owing to pressure effects, the method cannot be expected to give accurate results anyway), the internal boundary-layer thickness is well represented by the equation

$$
\frac{\delta}{\mathrm{z}_{02}}=\mathrm{a}\left(\frac{\mathrm{x}}{\mathrm{z}_{02}}\right)^{0.8}
$$

where $\mathrm{a}$ is a slowly varying function of $\mathrm{z}_{02} / \mathrm{z}_{01}$ which can be approximated by

$$
a=0.75-0.03 \ln \frac{z_{02}}{z_{01}}
$$

Although Elliott's velocity-profile assumption incorporates the essential features of the flow problem, it was found to yield results that were only roughly in agreement with observation. Attempts to improve Elliott's model without changing the method of approach have centered on improving the assumptions of Elliott. Plate and Hidy (1967) retained the velocity-profile assumptions of Elliott but dropped the assumption $\delta^{\prime}=0$. With $\delta^{\prime}$ small compared to $\delta$, it is possible to write Eq. 4.9 as

$$
\frac{\overline{\mathrm{u}}_{\delta}}{\mathrm{u}_{* 1}}=\frac{1}{\kappa}\left(\ln \frac{\delta}{\mathrm{z}_{01}}-\frac{\delta^{\prime}}{\delta}\right)
$$


They obtained a further simplification from integrating the momentum equation between $x=0$ and $x$. For this model the shear stress was set equal to an average value along $\mathrm{x}$, and the streamline passing through $\delta(\mathrm{x})$ was assumed to be a straight line. The advantage of the method of Plate and Hidy (1967) is that it readily permits the incorporation of pressure gradients and changes in roughness along the fetch. They also found that the method yielded results in good agreement with their laboratory data in the complex situation of wind blowing from a smooth shore onto water, in which case the wind gives rise to waves that determine the roughness of the surface. The comparison of the data with the theory is indicated in Fig. 4.4. The standard deviation $\sigma_{\mathrm{w}}$ of the water surface elevation is found to be proportional to $\mathrm{z}_{\mathbf{0 2}}$ and is, as shown in (b) of Fig. 4.4, a function of $\mathrm{x}$. Also, there existed a pressure gradient that was of some effect on the results of the calculations. The velocity distributions were found in good agreement with the experiments, as is seen in (a) of Fig. 4.4, until $x>2.5 \mathrm{~m}$, at which distance the theory loses its validity because the approach profile above the internal boundary layer begins to deviate from the assumed logarithmic form.

The results of the technique of Plate and Hidy for constant $z_{02}$ and zero-pressure gradient have been incorporated into a nomogram that can be used in similar situations. Introducing the abbreviations

$$
\begin{aligned}
& c=\ln \frac{\delta}{z_{02}} \\
& b=\ln \frac{z_{02}}{z_{01}}
\end{aligned}
$$

these results can be put into the form

$$
\begin{gathered}
\frac{\delta^{\prime}}{\delta}=\frac{b c^{2}}{D} \\
\frac{\mathrm{u}_{* 2}^{2}}{\mathrm{u}_{* 1}^{2}}=\frac{\mathrm{P}}{\mathrm{D}}+1 \\
\frac{\kappa^{2} \mathrm{x}}{\mathrm{z}_{02}}=\frac{\delta}{\mathrm{z}_{02}} \frac{\mathrm{N}}{\mathrm{P}}
\end{gathered}
$$

where $\mathrm{N}, \mathrm{P}$, and $\mathrm{D}$ are polynomials in $\mathrm{c}$ and $\mathrm{b}$ which are given by

$$
\begin{gathered}
D=c^{4}+c^{3}(b-1)+c^{2} \\
P=2 b c^{3}+c^{2}\left(3 b^{2}-2 b\right)+c\left(b^{3}-b^{2}\right)-b^{2} \\
N=c^{4} b+c^{3}\left(2 b^{2}-4 b\right)+c^{2}\left(b^{3}-6 b^{2}+2 b\right)-2 c b^{3}+2 b^{2}
\end{gathered}
$$




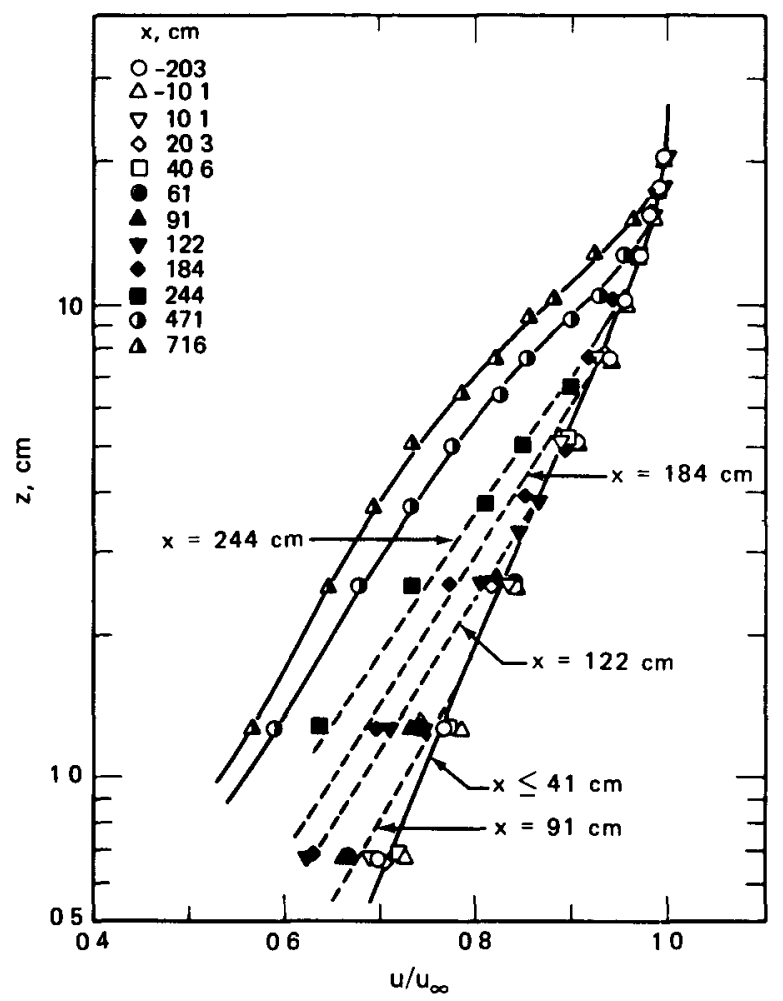

(a) Vertical distribution of average horizontal air flow over the smooth bottom and later over small wind waves The dotted lines represent theoretical profiles based on model of Plate and Hidy (1967)

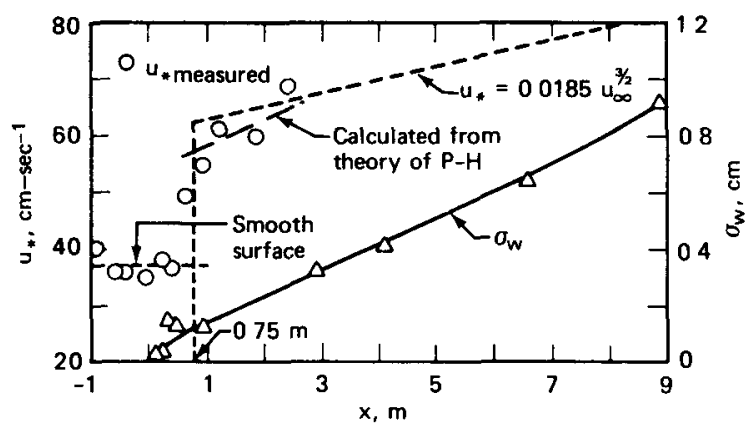

(b) Variation with fetch of the friction velocity $u_{*}$ and an average amplitude of surface waves, $\sigma_{w}$ Plate and Hidy (1967)

Fig. 4.4 Wind-profile development over small wind-generated water surface waves (from Plate and Hidy, 1967) 
Combinations of these polynomials were plotted for ready use in Fig. 4.5. From this figure it is comparatively simple to obtain a solution for a given situation. With given $\kappa$ and values of $\mathrm{z}_{0}$, we calculate $\kappa^{2} \times / \mathrm{z}_{02}$ and go into Fig. $4.5 \mathrm{a}$ from which we obtain, with suitable interpolation for $b$, the ratio $\delta / z_{02}$. With this ratio, enter Fig. $4.5 b$, get $\mathrm{N} / \mathrm{D}$, and calculate $u_{* 2}$ from Eq. 4.19. The whole process takes only a few minutes. By applying this method to Bradley's data for flow from smooth to rough, we find that $\delta$

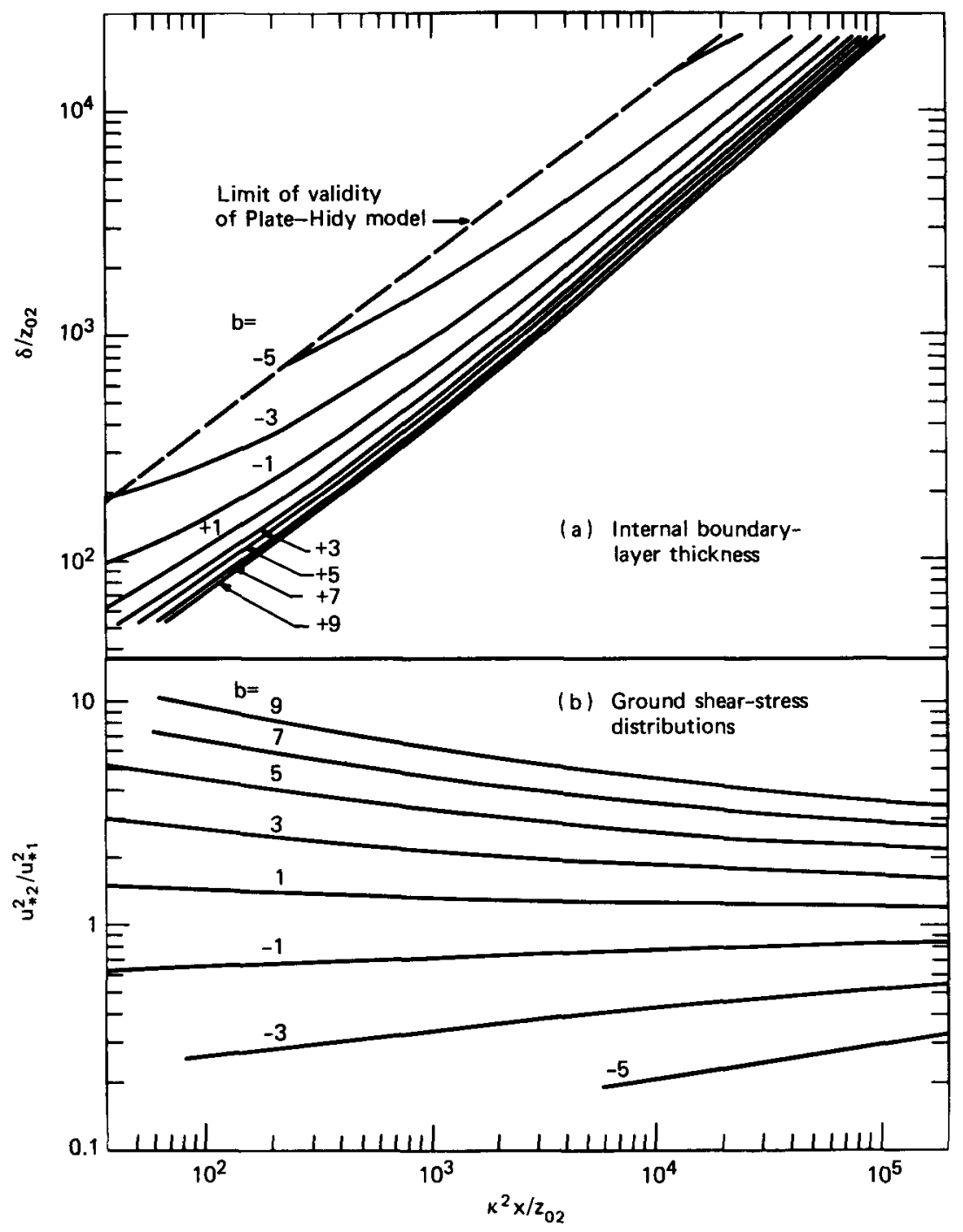

Fig. 4.5 Graphs for calculating internal boundary layers (from Plate and Hidy, 1967). 
is remarkably well predicted, as is seen in Fig. 4.2a, whereas initially the shear stress is slightly overestimated. At a fetch of $16 \mathrm{~m}$, however, the shear stress agrees very well with the measurements.

The modification of Elliott's method by Plate and Hidy probably yields satisfactory results as long as the blending region between the outer flow and the flow in the internal boundary layer is thin. This is not likely to apply for large distances $\mathrm{x}$ where the blending of the two profiles may extend over a substantial portion of the boundary layer, so that the blending function $f(z / \delta)$ becomes significant.

The introduction of the blending function does, however, require an additional condition for closing the set of equations. Panofsky and Townsend (1964) reasoned that the most unrealistic feature of Elliott's model is the condition, apparently implied by the logarithmic profile in the internal boundary layer, that the shear stress jumps discontinuously at $\mathrm{z}=\delta$ from $u_{* 2}^{2}$ to $u_{* 1}^{2}$. This criticism is, however, not entirely justified because it requires the mixing-length relation between shear stress and velocity profile:

$$
\tau=\mathrm{u}_{*}^{2}=(\kappa \mathrm{z})^{2}\left(\frac{\partial \bar{u}}{\partial \mathrm{z}}\right)^{2}
$$

which actually need not be invoked. Assuming it to be valid, however, and requiring continuity of shear stress, we impose an additional condition on the velocity distribution that can most simply be met by means of an additional function $\mathrm{f}\left(\mathrm{z} / l_{1}\right)$ given by the linear relation

$$
\mathrm{f}\left(\frac{\mathrm{z}}{l_{1}}\right)=\frac{\mathrm{u}_{* 1}-\mathrm{u}_{* 2}}{\kappa} \times \frac{\mathrm{z}}{l_{1}}
$$

This form was proposed by Panofsky and Townsend (1964). The addition of $\mathrm{f}\left(\mathrm{z} / \mathrm{l}_{1}\right)$ does not change the shape of the distribution very much; however, $l_{1}$ is the upper limit of the blending region between the outer and inner velocity profiles which is significantly different from the $\delta$ used by Elliott and by Plate and Hidy, where it denotes the intersection of the asymptotic forms of the inner and outer profiles. It is easily seen that the weakest link in the Panofsky-Townsend theory is the assumed form of the blending profile, other profiles leading to substantially different blending regions, and thus values of $l_{1}$, as Townsend (1965) and Taylor $(1969 \mathrm{a}, \mathrm{b})$ have shown. A realistic estimate of the width of the blending region requires a smoothing velocity distribution between the two intersecting asymptotic profiles that must be known with some accuracy. A rough guess as used by Panofsky and Townsend will not suffice.

The shear stress calculated by means of the Panofsky-Townsend model does not differ much from that found by Elliott's model. The calculations of $u_{* 2}^{2}$ for Bradley's data are found to be in slightly less satisfactory agreement with the data than the calculations using the curves of Fig. 4.6, but the reason for this lies probably more in the inclusion of the displacement height by Plate and Hidy which Panofsky and 


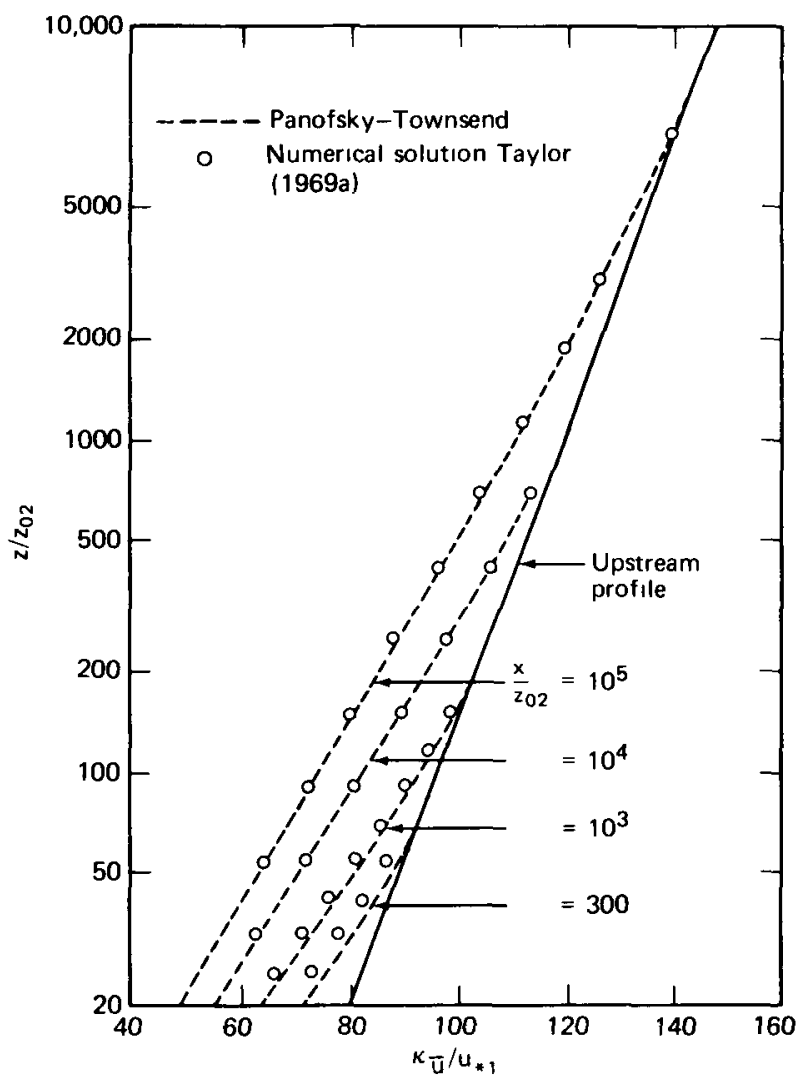

(a) Theoretical wind profiles, $b=5$

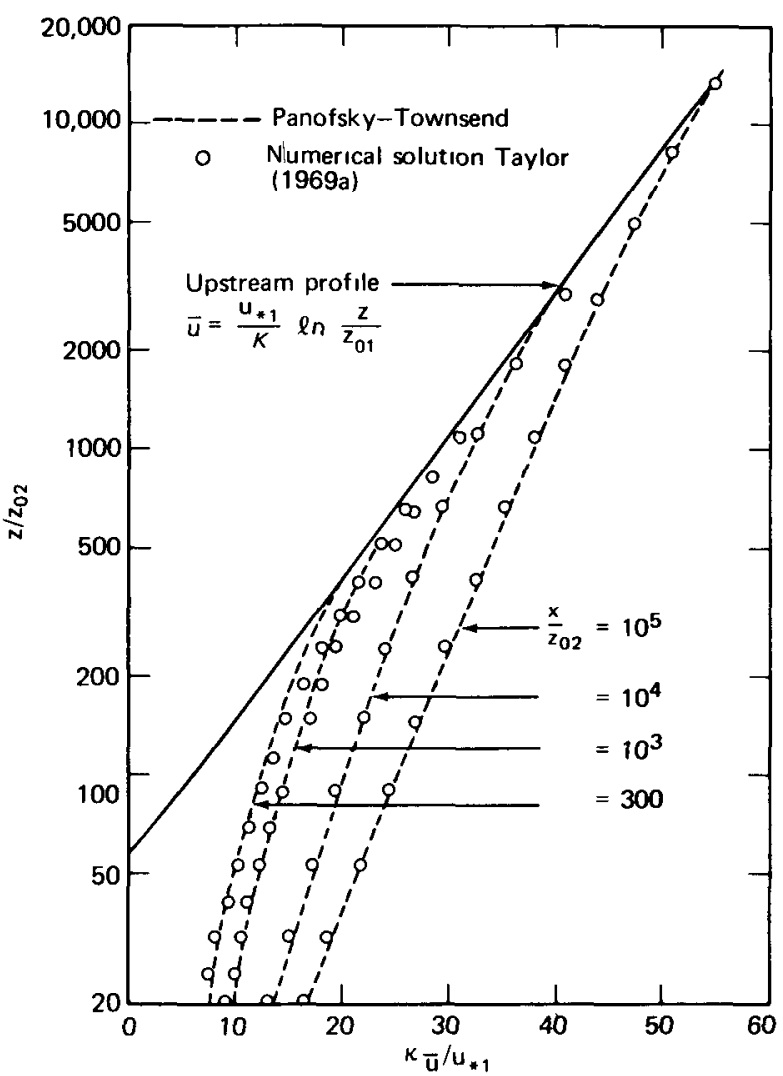

(b) Theoretical wind profiles, $b=-4$

Fig. 4.6 Comparison of numerical results from Taylor (1969a) with the Panofsky-Townsend model (from Taylor, 1969a) 
Townsend set deliberately equal to zero, and apparently the error resulting from this outweighs the slight improvement gained from the velocity profile

An attempt at improving the solution of Panofsky and Townsend was made by Taylor (1969a) who used a velocity profile that also gave continuity of the shear-stress gradient at the edge of the blending region This model was, however, not particularly successful, as Taylor has shown in the same paper

An approach based on the momentum equation was also given by Taylor (1962) Since he used the physically not particularly appealing proposition that $z_{0}$ is proportional to $\mathrm{u}_{*}$ to close his set of equations, his approach can serve only to emphasize the elegance of Elliott's model

\section{Methods Based on Similarity Arguments}

Townsend $(1965,1966)$ sought to improve the estımate for $f(z / \delta)$ by investigating the conditions under which it can be assumed to be a similarity profile (depending on no other length scale than $l_{1}$ ) Unlike the previously described models, Townsend's solution to the problem uses the equation of motion directly rather than in integrated form To obtain a solution, he assumed that the velocity downstream of the discontinuity consists of three parts the profile $\vec{u}_{1}(z)$ upstream of the discontinuity, a component $\Delta \overline{\mathrm{u}}$ that has to be added because of streamline displacement by $\delta^{\prime}(\mathrm{z})$, and the remaining difference $u^{\prime}$ between $\bar{u}_{2}$ and $\bar{u}_{1}$ which is due to an acceleration of the fluid in the internal boundary layer For displacement $\delta^{\prime}(\mathrm{z})$ values that are small compared with $\mathrm{z}, \Delta \overline{\mathrm{u}}$ can always be expressed by $\mathrm{u}_{*} \delta^{\prime}(\mathrm{z}) / \kappa \mathrm{z}$, obtained from the logarithmic $u_{1}$ profile by expansion in a Taylor series about $z$ Similarity enters through the assumptions that

$$
u^{\prime}=\frac{u_{0}}{\kappa} f(\eta)
$$

and

$$
\tau=u_{* 1}^{2}+\left(u_{* 2}^{2}-u_{* 1}^{2}\right) F(\eta)
$$

where $\eta=\mathrm{z} / l_{1}$, and $l_{1}$ and $\mathrm{u}_{0}$ are length and velocity scales, respectively, that depend on $\mathrm{x}$ only The functions $f$ and $F$ are assumed to depend on $\eta$ and not on $x$ The functions $\mathrm{f}$ and $\mathrm{F}$ are related through the mixing-length $\mathrm{Eq} 424$, which becomes

$$
\eta \mathrm{f}^{\prime}=\mathrm{F}
$$

where the prime denotes differentiation with respect to $\eta$ The equations of motion and continuity combine to yield for small $\delta^{\prime}(\mathrm{z})$

$$
-\eta \mathrm{f}^{\prime}=\mathrm{F}^{\prime}
$$


so that

$$
F=e^{-\eta}
$$

and

$$
f=E_{1}(-\eta)
$$

where $E_{1}$ is the exponential integral The scales $l_{1}$ and $\mathrm{u}_{0}$ are found from the conditions that the functions $f$ and $F$ are independent of $x$ and that the velocity profile must assume the logarithmic shape at the wall. The original calculations of Townsend had a small error in not yielding a velocity profile that exactly satisfied the latter boundary condition This inconsistency was removed by Blom and Wartena (1969) who gave the result for the length scale $l_{1}$

$$
\frac{l_{1}}{\mathrm{z}_{01}}\left(\ln \frac{l_{1}}{\mathrm{z}_{01}}-1\right)=2 \kappa^{2} \frac{\mathrm{x}}{\mathrm{z}_{01}}
$$

With the length scale known, the velocity scale is found to

$$
\mathrm{u}_{0}=\mathrm{u}_{* 1} \frac{\mathrm{b}}{1+\mathrm{M}}\left[\ln \frac{l_{1}}{\mathrm{z}_{02}}-\gamma+\left(1+\mathrm{M}^{-1}\right)^{-1}\right]^{-1}
$$

where

$$
\mathrm{M}=\left(\ln \frac{l_{1}}{\mathrm{z}_{01}}-1-\gamma\right)^{-1}
$$

and $\gamma=0577$ is Euler's constant. The shear stress downstream from the discontinuity can then be calculated from the relation

$$
u_{* 2}=u_{* 1}+u_{0}(1+M)
$$

The method of Townsend restores confidence in the meaning of the length $l_{1}$ as contrasted to the method of Panofsky and Townsend (1964). However, the Townsend method has the general shortcoming of the mixing-length assumption. Further improvement of the theory has come from numerical techniques in which more appropriate relations between shear stress and mean velocity distributions were used. The advantage of Townsend's method lies in the fact that, for the $\mathrm{x}$-dependent parameters, curves similar to those shown in $F_{1}$ 4.6 can be prepared which can be applied immediately to a given field situation. So it would be useful if improved shear relations could be found compatible with the similarity hypothesis of Townsend As yet such investigations have not been made 
The internal boundary-layer thickness downwind of a roughness discontinuity cannot be used directly as a measure of the distance below which the velocity profile represents local conditions only Because of the blending region, the velocity profile deviates significantly from a logarithmic form in the internal boundary layer, and theoretically it is nowhere equal to it An internal-layer height $h_{1}$ below which the loganthmic law holds must be smaller than either $\delta$ or $l_{1}$ A suitable definition of $h_{1}$ would be found by defining $h_{1}$ as that distance at which the actual velocity profile deviates by a specified percentage from the logarithmic profile A practical length based on the Townsend length $l_{1}$ has been proposed as $h_{1}=01 l_{1}$ With this definition the adjustment distances become very long For example, Blom and Wartena (1969) show that, for $a b$ of 2 , a distance of about $15 \mathrm{~km}$ is needed for an adjusted log profile over a height of $h_{1}=50 \mathrm{~m}$ to exist Such lengths make it virtually impossible to find anywhere fully developed turbulent boundary layers of thickness much less than $20 \mathrm{~m}$, and (if only for lack of steadiness) even a $10 \mathrm{~m} / \mathrm{sec}$ wind at a height of $50 \mathrm{~m}$ cannot be expected to keep very constant during the 25 min that 1 t takes to get from $x=0 \mathrm{~km}$ to $\mathrm{x}=15 \mathrm{~km}$

A method that is related to the similarity solutions of Townsend has been employed by Russian workers of the USSR Main Geophysical Observatory (Gandin, 1952, Dimitriev and Sokolova, 1954, see Panchev and Godev, 1970)

\section{Numerical Studies}

The internal boundary-layer problem has been treated numerically on high-speed computers by a number of workers (N1ckerson, 1968, Taylor, 1969a, Wagner, 1966, Onısh and Estoque, 1968) by means of the mixing-length relation These studies differ from each other mainly in the use of the boundary conditions and of the governing equations The most appropriate formulation of the internal boundary-layer problem is based on the set of Eqs 46 and 47 with the conditions at the boundary that the velocity profile must become logarithmic for $\mathrm{z} \rightarrow 0$ For this formulation, solutions have been obtained by Taylor (1969a) which show that the exact numerical solution has no significant advantages over the approximate treatments of the previous section In particular, Taylor found excellent agreement of the Panofsky-Townsend theory with his numerical calculations, except near the discontinuity, where neither method is valid This fact is illustrated in Fig 46

The crucial shortcoming of all these theories is a fallure to properly represent the relation between shear stress and velocity profile, a difficulty that can be overcome only by a more realistic representation of the effect of upstream conditions on the development of the shear stresses and the velocity profiles Whereas the mixing length is a local quantity, the shear stress develops over the whole length of the boundary layer, and only its rate of change is related to local conditions (Bradshaw, Ferriss, and Atwell, 1967) An equation for the shear stress that accounts for its development can be derived from the energy equation, Eq 243 of Chap 2 (with buoyancy omitted) 
Following Townsend (1956), a relation between the total turbulent kinetic energy at a point and the shear stress can be postulated

$$
\tau=-\overline{\mathrm{u}^{\prime} \mathrm{w}^{\prime}}=\mathrm{a}_{1} \overline{\mathrm{q}^{2}}
$$

where $a_{1}$ is a constant, equal to about 015 This relation is strictly empirical, but of surprisingly general validity, having been observed to hold in many different types of turbulent shear flows With Eq 436 the energy equation becomes

$$
\frac{1}{\mathrm{a}_{1}} \frac{\mathrm{d} \tau}{\mathrm{dt}}=\tau \frac{\partial \overline{\mathrm{u}}}{\partial \mathrm{z}}+\frac{\partial}{\partial \mathrm{z}}\left(\mathrm{w}^{\prime} \mathrm{q}^{\prime 2}+\mathrm{w}^{\prime} \frac{\mathrm{p}^{\prime}}{\rho}\right)-\epsilon
$$

The dissipation $\epsilon$ and the two diffusion terms must be related to $\tau$ and $\partial \bar{u} / \partial z$ to complete the analysis Bradshaw, Ferriss, and Atwell (1967) and independently Peterson (1969) have made the assumption that the dissipation is related to the shear stress as in a logarithmic equilibrum layer where production of shear stress equals dissipation, or

$$
\frac{\tau^{3 / 2}}{\mathrm{~L}}=\epsilon
$$

where $L$ is a characteristic length, corresponding to a characteristic time $t_{1}$ of Eq 287 in Chap 2 given by $\tau_{1}=\mathrm{L} / \tau^{1 / 2}$ In the constant-stress layer, $\tau=u_{*}^{2}$, and since near the wall dissipation and production are very nearly balanced (Bradshaw et al , 1967), it follows that near the wall $\mathrm{L}=\kappa \mathrm{z}$, and this form has been assumed by Peterson (1969) to be valid throughout the boundary layer This assumption is rather difficult to accept, on physical grounds, and if erroneous may also lead to nonrealistic results in view of Bradshaw's observation that the functional form of $L$ significantly affects the results

The pressure-diffusion term in Eq 437 was neglected, both by Peterson and by Bradshaw et al The latter also neglected the energy-diffusion term Peterson argued that the energy diffusion is of the gradient type

$$
\overline{w^{\prime} q^{\prime 2}}=-K \frac{\overline{\partial q^{\prime 2}}}{\partial z}
$$

It is logical to assume that the diffusion coefficient is the same as for momentum

$$
\overline{u^{\prime} w^{\prime}}=-K_{M} \frac{\partial \bar{u}}{\partial z}
$$

Apparently the exact form of the energy diffusion term is of no particular importance, and the assumptions of Eqs 439 and 440 are quite reasonable Inserting the forms for $\epsilon$ and the diffusion term into Eq 437 yields, for a steady flow, 


$$
\overrightarrow{\mathrm{u}} \frac{\partial \tau}{\partial \mathrm{x}}+\overline{\mathrm{w}} \frac{\partial \tau}{\partial \mathrm{z}}=0.16 \tau \frac{\partial \overline{\mathrm{u}}}{\partial \mathrm{z}}+\frac{\partial}{\partial \mathrm{z}}\left(\tau \frac{\partial \tau}{\partial \mathrm{z}} / \frac{\partial \overline{\mathrm{u}}}{\partial \mathrm{z}}\right)-04 \frac{\tau^{3 / 2}}{\mathrm{z}}
$$

where a Karman constant of 0.4 has been used

Equation 441 is the third equation required to solve the internal boundary-layer problem previously mentioned. Numerical solutions obtained by Peterson differ from those obtained on the basis of mixing theories by showing a transitional profile in the blending region with double curvature, the profile having a gradient which overshoots that of the two asymptotic logarithmic profiles This is evident in Fig. 4.7 where the nondimensional gradient calculated by Peterson

$$
\phi=\frac{04 \mathrm{z}}{\tau^{1 / 2}} \frac{\partial \overline{\mathrm{u}}}{\partial \mathrm{z}}
$$

has been plotted as a function of $\mathrm{z}$ which is made nondimensional by dividing it through the internal boundary-layer thickness $\delta$ (defined as that distance from the ground at which the shear stress $\tau=0.99 \mathrm{u}_{* 1}^{2}$ ). The types of profiles corresponding to

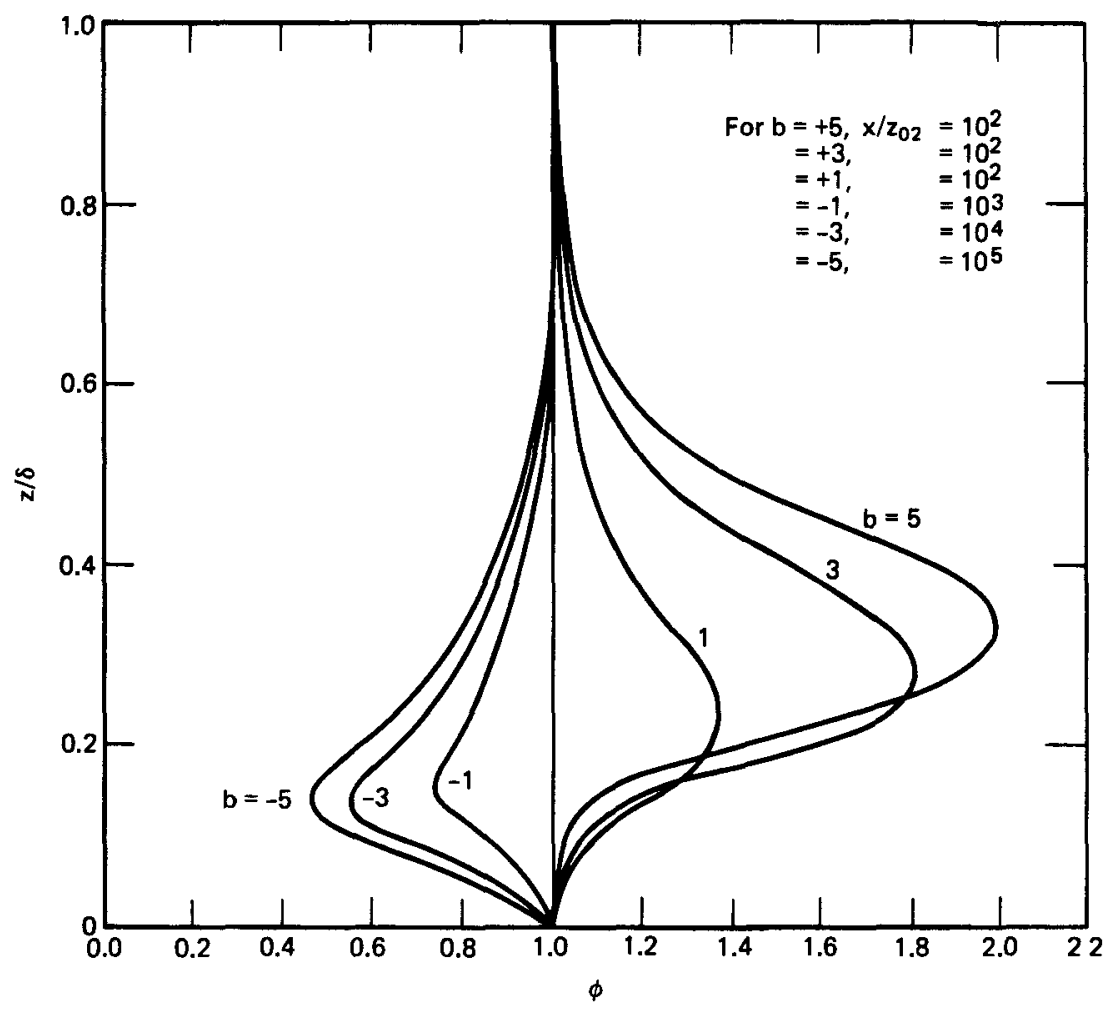

Fig. 4.7 Nondimensional wind-velocity gradient as a function of $z / \delta$ (from Peterson, 1969) 
this result are similar to the ones observed by Bradley (1968) Also, the shear-stress distributions at the ground computed by Peterson are in better agreement with Bradley's experimental results than any of the other theories, as is shown in Fig 42.

Other attempts have been made to develop a constituent equation for the shear stress on the basis of rate equations, but we mention only that of Smith (1967) who developed a conservation equation for the eddy diffusivity $K$, on rather questionable physical grounds.

\section{Further Developments in the Theory of Internal Boundary Layers}

The problem of the internal boundary layer has been developed as far as is justifiable for the change downstream of a logarithmic velocity profile, and further investigations along this line must either incorporate more elaborate upstream velocity distributions or must include added terrain features or boundary conditions of higher degrees of complexity. Thus the model of Townsend (1966) was extended by Blom and Wartena (1969) to more than one discontınuity in wall roughness, and the problem of the thermally stratified boundary layer was treated by Blackadar et al (1967), Taylor (1970, and Panchev and Godev (1970) who also refer to work on the subject by Nadejdina $(1964,1966)$ Basically the problem was treated by simultaneously solving the heat equation (Eq. 240 of Chap. 2) and Eqs. 4.1 to 4.3 and by connecting profiles of temperature and velocity through suitable exchange coefficients for heat flux and shear stress. Typical is the system of equations used by Taylor (1970) who adds to the system of equations (Eqs. 4.1 to 4.3 ) the heat-flux equation, Eq. 240 of Chap 2 (with $\mathrm{d} \theta / \mathrm{dt}$ instead of $\partial \theta / \partial \mathrm{t}$ and with conduction and dissipation neglected)

$$
\overrightarrow{\mathrm{u}} \frac{\partial \theta}{\partial \mathrm{x}}+\overrightarrow{\mathrm{w}} \frac{\partial \theta}{\partial \mathrm{z}}=-\frac{\partial \mathrm{H}}{\partial \mathrm{z}}
$$

and the Businger-Dyer formulation of the temperature and velocity profiles in unstably stratified flow

$$
\begin{gathered}
\tau^{1 / z}=\frac{\kappa z}{\phi_{M}} \frac{\partial \bar{u}}{\partial z} \\
H=-\frac{\kappa z \tau^{1 / 2}}{\phi_{\mathrm{H}}} \frac{\partial \theta}{\partial z}
\end{gathered}
$$

with

$$
\phi_{\mathrm{M}}=\left(1-\beta \frac{\mathrm{z}}{\mathrm{L}}\right)^{-1 / 4}
$$


and

$$
\phi_{\mathrm{H}}=\phi_{\mathrm{M}}^{2}
$$

with $\mathrm{L}$ assumed to be given by

$$
\mathrm{L}=-\bar{\theta}_{1} \frac{\mathrm{u}_{* 1}^{2}}{\mathrm{~kg}} \frac{\mathrm{u}_{* 1}}{\mathrm{H}_{01}}
$$

corresponding to the upstream condition, or for neutral upstream conditions

$$
\mathrm{L}=-\bar{\theta}_{1} \frac{\mathrm{u}_{* 1}^{2}}{\kappa \mathrm{g}}\left(\bar{\theta}_{2}-\bar{\theta}_{1}\right)
$$

The numerical results obtained from this model await verification by field experiments, and the validity of the basic assumptions requires laboratory checking. The presently available data by Dyer and Crawford (1965) are not sufficient for this purpose.

It can be expected that internal boundary-layer theory applies not only to the case of slight stratification, in which the log-linear law is approximately valid, but also to conditions of free convection. A remarkable feature of internal boundary layers with thermal "stratification is the possibility of finding free convection layers capped by a layer with unstable temperature gradients. This situation can occur when neutrally stratified but cold air moves onto a warm surface and is heated sufficiently from below so that a free-convection layer exists above the local superadiabatic layer. In the free-convection layer, the temperature is roughly constant but warmer than in the air above; consequently a heat flux to the outer air and an unstable gradient of temperature exist. Evidence for such a situation exists, for example, in temperature profiles (unpublished) taken in a wind tunnel by S. Arya. The structure of such layers is of some interest in environmental studies because they might be responsible for trapping air pollutants in environments where ordinary inversions cannot be found.

From a complete theory of the internal boundary layer, we must be able to predict the effects of simultaneous changes of heat flux and roughness, such as are encountered by sea breezes or similar flows. The internal boundary-layer model provides the lower boundary conditions for more general sea-breeze models, such as the ones that are studied by Estoque $(1961,1962)$. In nature such problems are further complicated by the diurnal cycle in heating of the ground and by the differences in heat-transfer characteristics of complex natural terrain. An illustration of this is given in Fig. 4.8 (from Carson and Nelson, 1969) in which temperature distributions over Chicago during a breeze from the cold water of Lake Michigan are shown for three different locations. Because the lake water is colder than the higher air masses, a strong inversion exists over the lake. Upon reaching the shore the inversion is lifted above the warmer ground. Further inland, not only is the inversion raised but the ground temperature increases also. Furthermore, since the profiles were taken 


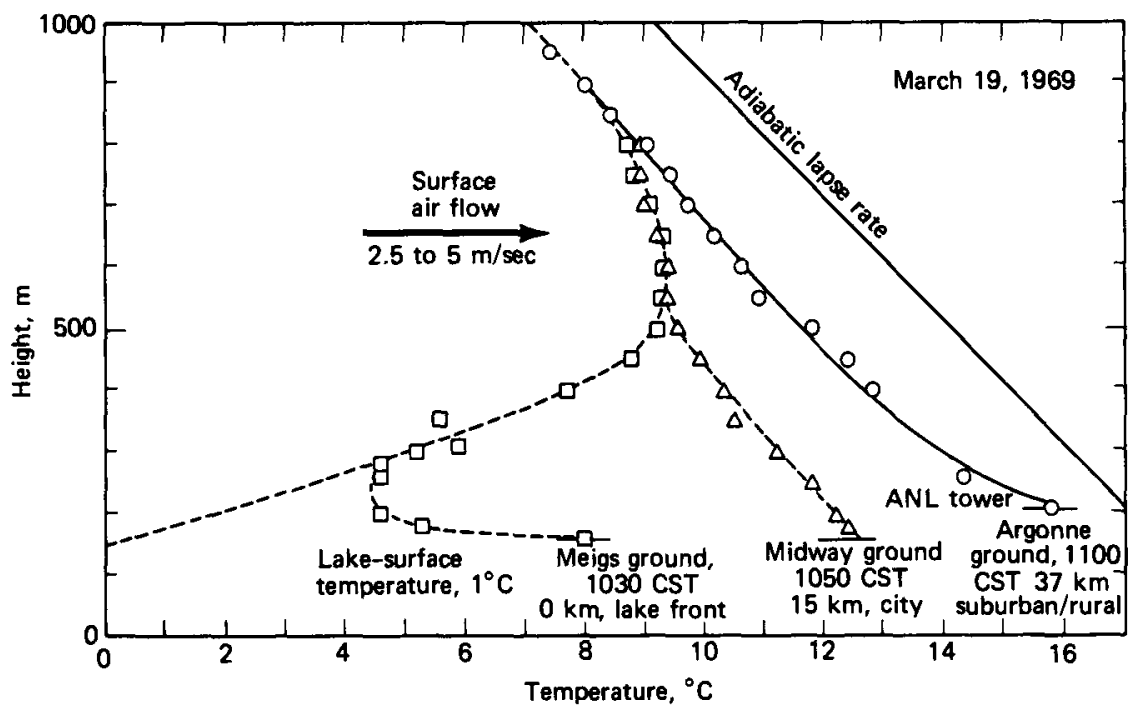

Fig. 4.8 Example of a thermal internal boundary layer: vertical temperature profiles in Chicago during an onshore wind from Lake Michigan, Mar. 19, 1969 (from Carson and Nelson, 1969). $\Delta$, Midway Airport. $\bigcirc$, Argonne National Laboratory. $\square$, Meigs Airport.

1. Undisturbed boundary layer (outer layer)

2 , Region of hill influence (middle layer)

3. Region of reestablishing boundary layer (inner layer)

4 , Blending region between middle and outer layer

5 , Blending region between inner and middle layer

6. Standing eddy zone

7. Potential outer flow

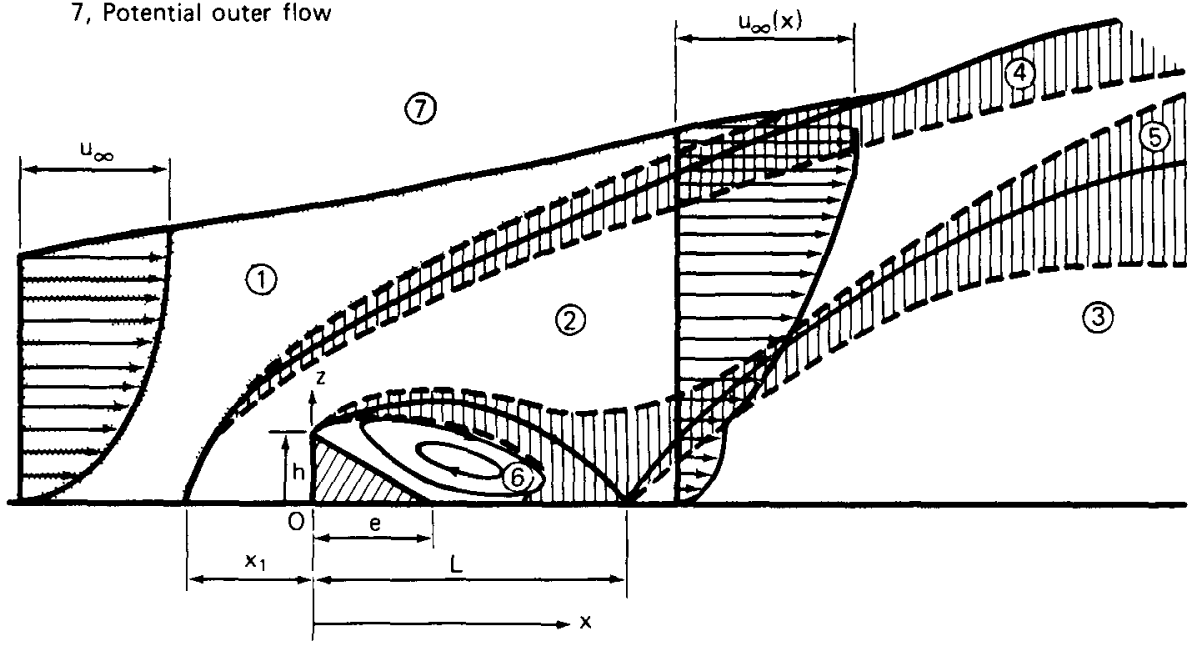

Fig. 4.9 The flow zones of a boundary layer disturbed by a shelterbelt (from Plate and Lin, 1965). 
almost simultaneously, the arr masses seen at the different stations are not the same. Thus, although the situation shown in Fig. 49 indicates unmistakably the existence of an internal boundary layer, at present we cannot calculate wind or temperature profiles for it. A more thorough discussion of the sea breeze over Lake Michigan has been given by Moroz (1967).

\section{SHELTERBELTS*}

The complexity of the flow around a wind shelter is evident from the schematic situation shown in Fig. 4.9. A boundary-layer flow is approaching a wedge-shaped obstruction that has been placed on a flat plate. No less than seven flow zones of different aerodynamic behavior can be distinguished In zone 1 the flow field is mostly determined by the conditions in the undisturbed boundary layer far upstream from the wedge. In zone 2 the flow field is displaced and distorted owing to the presence of the wedge, with the lower boundary of zone 2 given by the separation-induced shear layer that starts at the edge of the wedge and forms the transition to the highly retarded flow in zone 3 When the wedge is solid, backflow may occur, leading to a separation bubble with a reattachment point, at a distance L downstream from the wedge. Downstream of the reattachment point, the flow is again in the direction of the mean wind. In layer 5 the flow gradually increases in velocity until at some large distance the "inner layer" 5 has blended with the outer flow, and a new and thicker boundary layer is formed which adjusts to the local boundary conditions at the ground until the effect of the obstruction can only be inferred by comparing the boundary-layer thickness with the thickness that would have existed if the wedge had not been there. The flow in the region downstream from reattachment is that of the adjustment of an initial velocity profile to the local boundary conditions and can be determined, in principle, from an initial profile downwind of reattachment by methods of boundary-layer calculations, as has been done by Plate (1967) for distances larger than $35 \mathrm{~h}$, where $\mathrm{h}$ is the height of the obstruction. At present, however, such analyses depend on empincal observations and assumptions, and further research is needed to eliminate the empirical constants Here we shall be concerned mostly with zones 2 and 6 , which are most important to the sheltering problem

The most common design requirement for a shelterbelt is that the wind be reduced below the dangerous level over a maximum distance behind the shelterbelt. Discovering the shelter that does this most efficiently has been the objective of much of the research on shelterbelts. It is an enormously complex analytical problem since it requires a solution of the full turbulent Navier-Stokes equation for a complete treatment. It is not surprising that most shelterbelt research has been done experimentally and by trial and error, by evaluating the wind reduction behind existing belts. Most often quoted are the results of Nagelı (1941) who obtained an

\footnotetext{
*This section is based on a paper that is to be published in the Journal of Agricultural Meteorology, 1971
} 
optimum solution of sorts by showing that a screen having medium density reduced the velocity by at least $20 \%$ over a larger distance than either a very dense screen or a screen with very high porosity. Similar results were found in a wind tunnel by Blenk and Trienes (1956). A generally accepted optimum shape has not been found, and most field research has been conducted on existing shelterbelts for a post facto assessment of their effect. Reviews of field results are given by Geiger (1965) and van der Linde (1962).

The only theoretical treatment of the shelterbelt problem known to the writer was given by Kaiser (1959) who assumed that sheltering results from diffusion of the momentum defect downwind from the shelter as if it were a passive scalar. This model is physically unrealistic and somewhat oversimplified, yet it does point to the decisive role that the drag plays in the shelter problem. It also leads to a prediction of velocity profiles that are described by the error function over a part of the air layer in the sheltered region. It would indeed be too much to expect an analytical solution that covers all details of the shelterbelt flow. However, many aspects of the flow field are very similar to well-known aerodynamic situations, and in this chapter we shall concentrate on these aerodynamic features. They form the building blocks from which ultimately a model for calculating the effect of shelters must be constructed.

\section{The Flow in the Sheltered Region Behind a Solid Shelter}

A detailed study of the flow field directly downwind of the obstruction has been made, for a solid shelter, by Chang (1966) who used experimental data obtained in a wind tunnel at Colorado State University. The upper part of some of his mean velocity profiles is shown in Fig. 4.10a. The velocity profiles are poorly defined in the separation bubble below the parts of the profiles shown because large pressure gradients exist as indicated in Fig. $4.10 \mathrm{c}$ which distort pitot-static tube measurements and because the low mean velocities, at high turbulence levels, cast serious doubts on measurements obtained with hot wire anemometers in this region. Similar measurements behind obstacles consisting of a fence have been given by Good and Joubert (1967) and by Mueller, Korst, and Chow (1963) for an obstacle with a quarter-circle cross section that faces the flow with the round surface. Measurements behind a sharp-edged plate placed perpendicular to an airstream were reported by Arie and Rouse (1956). All these measurements have in common that they show a very rapid change of velocity across a curve that can be identified as the location of all velocities equal to one-half the velocity $u_{\infty}$ outside the boundary layer. This curve is identical to the separation streamline near the upper edge of the shelter. It will be taken as the intrinsic $x$-axis, with the $z$ coordinate measured from it. The flow field has all the characteristics of the flow that results if an upper airstream with initially uniform velocity $u_{\infty}$ is joined along the separation streamline with a lower stream of zero velocity, or, in the case of a porous shelter, with velocity $u_{b}$ through the shelter, with a lower stream of velocity $u_{b}$. The solution for this flow situation is well known (see 


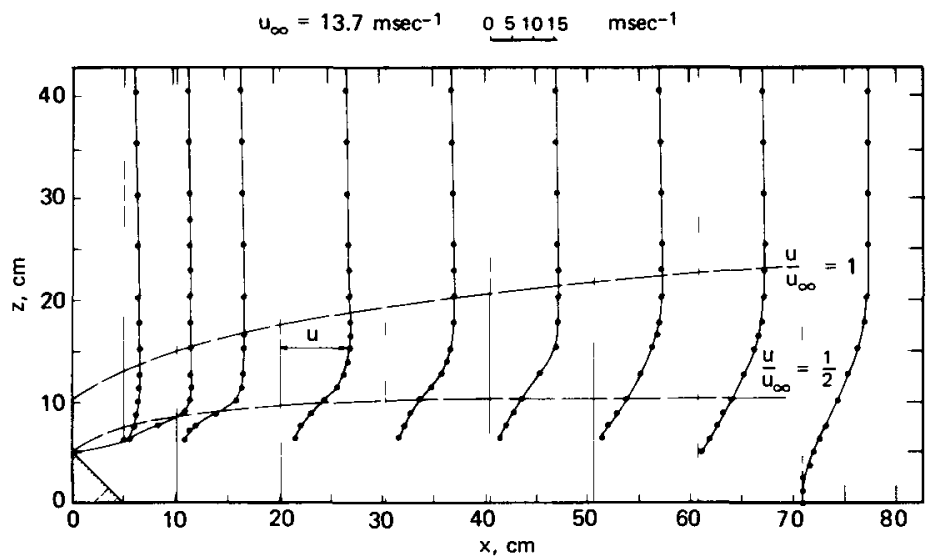

(a) Mean velocities



(b) Turbulence characterıstıcs

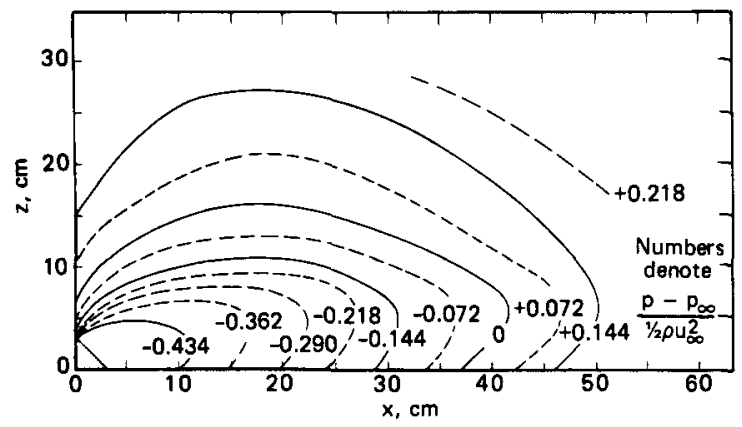

(c) Pressure

Fig. 4.10 Experimental data on the flow field downwind of a shelter (wind-tunnel results of Chang, 1966). (a) Mean velocities. (b) Turbulence characteristics. (c) Pressure. 


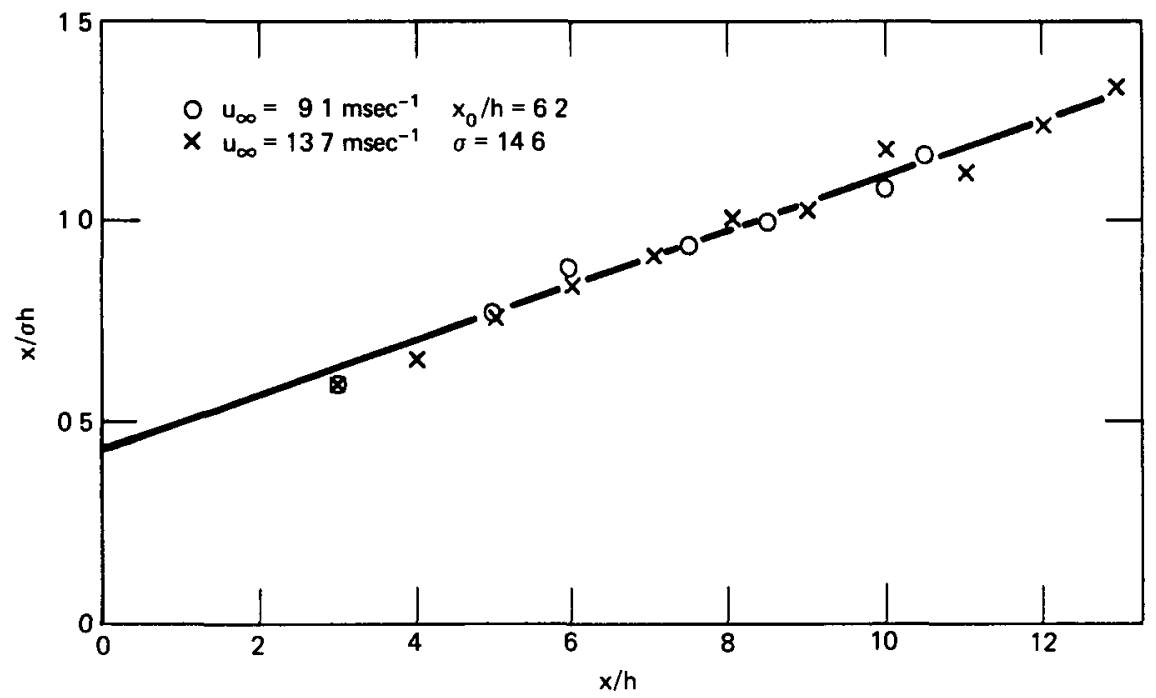

Fig. 4.13 The experimental values $\mathrm{x} / \mathrm{oh}$ vs $\mathrm{x} / \mathrm{h}$ from mean velocity data

How the wind profile develops near the ground downwind of the shelter is another aspect of flow with a porous shelter which at present is not fully understood Evidently the analytical model of the two parallel currents loses its usefulness as soon as the presence of the lower boundary makes itself felt on the blending region between the currents On the other hand, for the large values of $u_{b} / u_{\infty}$ the coefficient $\lambda$ de creases so that blending between inner and outer flow proceeds at a slower rate But large values $u_{b} / u_{\infty}$ mean little sheltering and large velocity gradients near the ground We can see that there exists an optımum porosity at which $u_{b}$ is a minimum over the longest distance As already mentioned, such an optimum has indeed been found in natural environments, as shown in Fig 4 14, which is from Nagelı (1941) The sheltering was determined by measuring the actual wind velocity at a height of $14 \mathrm{~m}$ and dividing it by the velocity that would have existed in the absence of a shelter A shelterbelt of medium density showed the best results Systematic wind-tunnel tests by Jensen (1954) and by Blenk and Trienes (1956) showed a maximum sheltering to be associated with porosities (defined as percentage of open area in the total area of the screen) of from 35 to $50 \%$

\section{Displacement of the Separation Streamline}

The error-function profile is a realistic representation of the mean velocity distribution in the neighborhood of the separation streamline But the development was assumed to take place along the curve given by $u / u_{\infty}=05$ which was found experimentally A prediction of the flow field between the shelter and the 


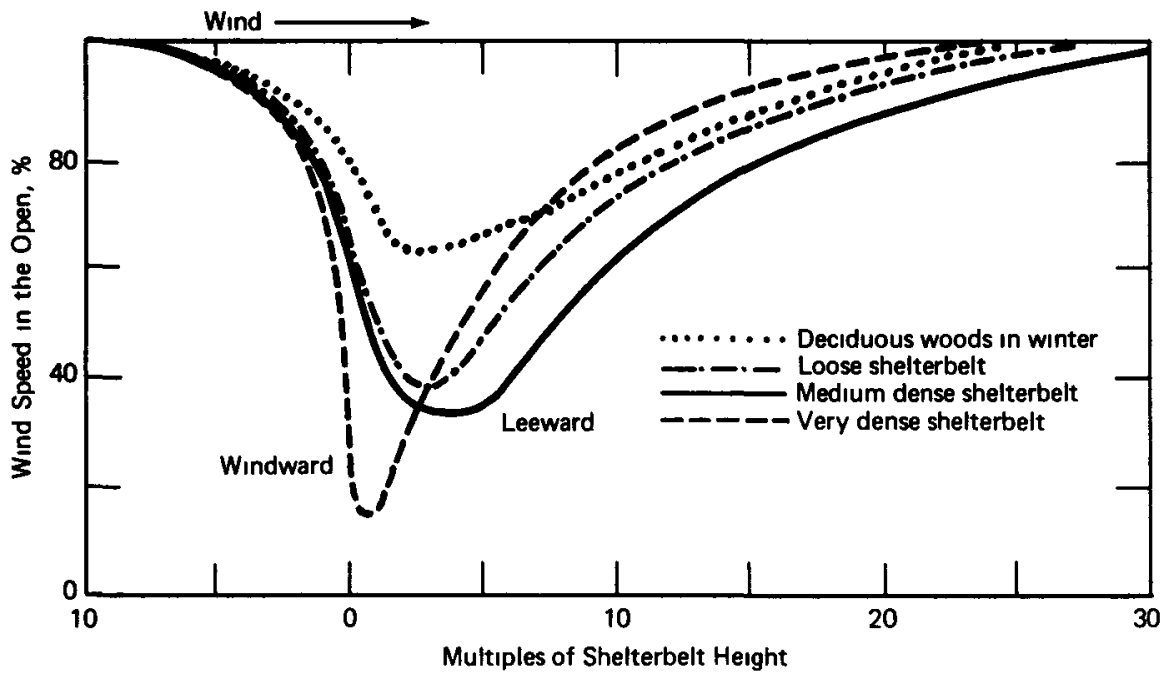

Fig. 4.14 Shelterıng at different porosities, according to Nagelı (1941)

reattachment point is possible only if the location of this curve can be determined with only a knowledge of the velocity distribution in the undisturbed boundary layer and of the characteristics of the shelter At present such a prediction is not possible We may, however, gain some understanding of how this location depends on the drag on the shelter and on the base pressure behind it, $1 \mathrm{e}$, how it depends on the pressure distribution about the shelter

The effect of the shelter on the separation streamline may be considered in an analysis that neglects the effect of the lower boundary except by letting the ground form a streamlıne The flow shown in Fig 415 represents a simplified model of this situation For generality, a porous screen is considered The screen introduces a momentum sink that leads to a deceleration of the flow upstream from $u_{\infty}$ to $u_{b}$, where $u_{b}$ is the velocity through the shelter, and to an upward deflection of the streamlines as a result of mass conservation A measure of the streamline displacement, or of the vertical extent of the sheltered area, can be obtained by applying the conservation of mass and of momentum principles to the free-body diagram indicated by A, B, C, and D in Fig 415 The change in momentum must be balanced by the external forces in the $\mathrm{x}$ direction If the effect of blending is neglected, which is likely to be permissible near the screen, then the sum $\Sigma_{C D}$ of momentum fluxes and forces on $\mathrm{CD}$

$$
\Sigma_{\mathrm{CD}}=\left(\rho \mathrm{u}_{\mathrm{b}}^{2}+\mathrm{p}_{\mathrm{b}}\right) \mathrm{h}^{*}+\left(\rho \mathrm{u}_{\infty}^{2}+\mathrm{p}_{\infty}\right)\left(l-\mathrm{h}^{*}\right)
$$

where the subscript $\infty$ refers to conditions in the undisturbed flow far from the lower boundary, the subscript $b$ refers to conditions slightly behind the screen, and $p$ is the 
pressure For definitions of the length parameters in Eq 4.54, see Fig 4.15 For AB one gets

$$
\Sigma_{\mathrm{AB}}=+\left(\rho \mathrm{u}_{\infty}^{2}+\mathrm{p}_{\infty}\right) l
$$

The momentum flux across $\mathrm{BC}$ is $+\rho \mathrm{u}_{\infty} \times \mathrm{Q}_{\mathrm{BC}}$ where $\mathrm{Q}_{\mathrm{BC}}$ is the volume flux across $B C$, which evidently is the difference in volume flux into $A B$ and out of $C D$, so that

$$
\Sigma_{\mathrm{BC}}=-\rho \mathrm{u}_{\infty}\left(\mathrm{u}_{\infty}-\mathrm{u}_{\mathrm{b}}\right) \mathrm{h} *
$$

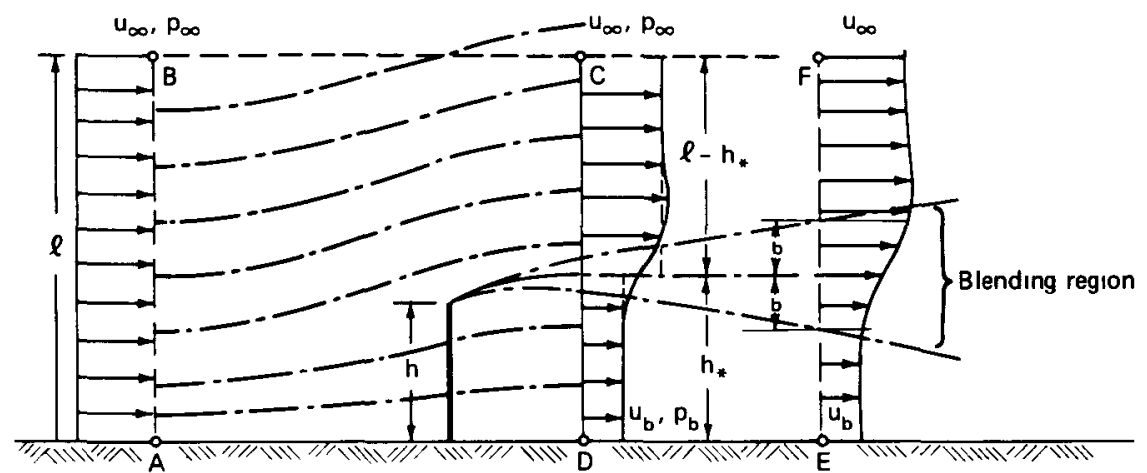

Fig. 4.15 Momentum balance with boundary-layer-type velocity profile neglected

The additional external force acting on the fluid is the drag on the screen, so that Newton's second law leads to

$$
\mathrm{D}=-\Sigma_{\mathrm{CD}}+\Sigma_{\mathrm{AB}}+\Sigma_{\mathrm{BC}}
$$

The solution $\dagger$ of this equation for $h^{*} / h$ ss, with Eqs 454 to 456

$$
\frac{h^{*}}{h}=\frac{C_{D}}{Q+\frac{2 u_{b}}{u_{\infty}}\left(1-\frac{u_{b}}{u_{\infty}}\right)}
$$

$\dagger$ A similar derivation for a solid plate in a stream of fluid has been given by Reichardt (as quoted by $\mathrm{G}$ Birkhoff, in Hydrodynamics, Dover Publications, Inc, 1950) who applied these 1deas to predict the width of a cavitation bubble 
where $C_{D}$ is the drag coefficient and $Q$ is the base pressure coefficient, defined by

$$
C_{D}=\frac{D}{1 / 2 \rho u_{*}^{2} h}=\frac{2 \int_{0}^{h}\left(p_{\text {front }}-p_{b}\right) d z}{\rho u_{\infty}^{2} h} ; Q=\frac{p_{\infty}-p_{b}}{1 / 2 \rho u_{\infty}^{2}}
$$

When $u_{b}$ is equal to zero, the height $h^{*}$ of the sheltered region is proportional to $\mathrm{C}_{\mathrm{D}} \mathrm{h} / \mathrm{Q}$. This result is in good agreement with experimental data, both for a flat plate in a free stream, like the result of Arie and Rouse (1956) and for a plate in a boundary layer, as shown by Plate (1964). Plate found that $C_{D}=1.65 \mathrm{Q}$ and $\mathrm{h} *=1.67 \mathrm{~h}$, the latter result based on data of Nagabhushanaiah (1961). Apparently Eq. 4.58 is an approximation only since both the approach velocity profile and the diffusion of the interface between flow in the shelter and outside the shelter are neglected. More accurate results by Good and Joubert (1968) have shown that $\mathrm{C}_{\mathrm{D}}=1.82 \mathrm{Q}$; however, these writers have not given parallel results on $h^{*}$.

When $u_{b} \rightarrow u_{\infty}$, the pressure at both front and back of the screen approaches $p_{\infty}$, and thus both $\mathrm{C}_{\mathrm{D}}$ and $\mathrm{Q}$ approach zero at the same rate, so that in the limit $\mathrm{u}_{\mathrm{b}}=\mathrm{u}_{\infty}$; $h^{*}=h$, as it must. For small values of $u_{b} / u_{\infty}$, it is likely that $h^{*} / h$ decreases below the value at $u_{b} / u_{\infty}=0$ because the ratio $C_{D} / Q$ remains roughly constant. Consequently a porous screen results in a smaller height of the sheltered volume as compared to a solid screen.

\section{The Drag on the Shelter}

The drag on the shelter is governed not only by the shape and porosity of the shelter but also by the aerodynamic characteristics of the approaching boundary-layer flow. This has been made clear in the researches of Plate (1964) and Good and Joubert (1968). The latter have shown that, if the height of the (solid) shelter is less than 0.5 times the thickness of the boundary layer (as is usual for real shelterbelts), then the approaching boundary-layer flow sets the scales for modeling all the features of the problem. Thus if the velocity profile that would exist at the location of the shelter in the absence of the shelter is logarithmic and described by a profile of the form

$$
\frac{\mathrm{u}}{\mathrm{u}_{*}}=\frac{1}{\kappa} \ln \frac{\mathrm{z}}{\mathrm{z}_{0}}
$$

where $\kappa$ is von Karman's constant $(\sim 0.4)$, then the shear velocity $u_{*}$ sets the velocity scale for the drag, and the roughness height $z_{0}$ of the surface configuration sets the length scale. Consequently we may expect to find that a drag coefficient $\mathrm{C}_{*}$ of the shelterbelt based on $u_{*}$

$$
\mathrm{C}_{*}=\frac{\mathrm{D}}{1 / 2 \rho \mathrm{u}_{*}^{2} \mathrm{~h}}
$$


is a universal function of $\mathrm{h} / \mathrm{z}_{0}$. The experiments of Good and Joubert (1968) were made with a smooth surface only. In that case there is no characteristic length of the rough surface, and the only length that can be used to represent the surface characteristics must be based on the kinematic viscosity $\nu$ and the shear velocity $u_{*}$. Consequently it is found that

$$
\mathrm{C}_{*}=\mathrm{f}_{1}\left(\frac{\mathrm{u}_{*} \mathrm{~h}}{\nu}\right)
$$

for a smooth surface (Good and Joubert, 1968), and for a rough surface we may put

$$
\mathrm{C}_{*}=\mathrm{f}_{2}\left(\frac{\mathrm{h}}{\mathrm{z}_{0}}\right)
$$



Fig. 4.16 Drag coefficients of fences in thick boundary layers.

The functions $\mathrm{f}_{1}$ and $\mathrm{f}_{2}$ are, of course, dependent also on shelter geometries. As yet only Eq. 4.62 has been tested experimentally and only for sharp-edged shelters, with the results shown in Fig. 4.16.

In Fig. 4.16 the data are fitted by a straight line, whose equation has been given by Good and Joubert (1968) to

$$
\mathrm{C}_{*}=277 \log \frac{\mathrm{u}_{*} \mathrm{~h}}{\nu}-268
$$


Analogously, we expect to find, for a fully rough surface

$$
\mathrm{C}_{*}=\mathrm{A} \log \frac{\mathrm{h}}{\mathrm{z}_{0}}+\mathrm{B}
$$

The results in Eqs. 4.64 and 4.65 have an important consequence for modeling a solid shelter in a wind tunnel. Identical drag coefficients are obtained if, for a rough approach flow, the ratio $\mathrm{h} / \mathrm{z}_{0}$ is kept constant-i.e., if the geometries of the shelter are identical in model and prototype and if $h / \delta<0.15$ in the model-which implies

$$
\frac{\mathrm{h}_{\text {model }}}{\mathrm{h}_{\text {prototype }}}=\frac{\mathrm{z}_{0 \text { model }}}{\mathrm{z}_{0 \text { prototype }}}
$$

Consequently, in selecting a profile of the mean velocity distribution upstream of the shelter, we determine the scale factor for the experiments. It should be noted that Blenk and Trienes (1956) did not attempt to scale Nageli's (1941) flows in this manner; they needed an arbitrary conversion factor of 0.5 for reducing the downwind distance to make their experimental data conform to the results of Nageli. This is a clear indication that modeling was not properly accomplished.

How the drag coefficient of a porous shelter is related to the approach velocity profile can now only be surmised. It should be defined more suitably by using $u_{b}$ as reference velocity:

$$
C_{p}=\frac{D}{1 / 2 \rho u_{b}^{2} h}
$$

in which $C_{p}, D$, and $u_{b}$ are unknowns whose interrelations are not known. The drag coefficient $C_{p}$ depends on the porosity and also on the Reynolds number of the elements forming the shelter. For screens consisting of wires, some systematic experiments have been made to determine this dependency by stretching screens across the whole cross section of a wind tunnel and measuring the pressure drop at a given approach velocity. Typical are the investigations of Schubauer et al. (1950). In a natural shelter it is usually not possible to specify the porosity and the sizes of the shelter elements, and all estimates of the drag must rely either on direct measurement of the flow near similar installations or on guesses. Such measurements must naturally be made at a time during the growing season which corresponds to the time shelter is required by the crops; otherwise differences in foliage or shelter growth might be sufficient to render valueless any conclusions based on the experiment.

\section{The Pressure Effects}

The main difficulty in predicting drag forces on the shelter stems from our inability to determine the base pressure, i.e., the pressure at the back of the shelter. It 
1s obvious from Eq 459 that the larger the difference between pressure on the front and the base pressure, the larger the drag The pressure on the front of the shelter is generally determined, for a solid shelter, by the flow pressure of the approaching flow on the face of the shelter, independent of the shape of the shelter This is strictly true for a plate suspended in the free stream, with velocity $u_{\infty}$ and pressure $p_{\infty}$, of a wind tunnel The pressure on the front in that case can be calculated from inviscid flow theory (Arie and Rouse, 1956), and the maximum pressure at the center of the plate is found to be exactly equal to the stagnation pressure $\mathrm{p}_{\mathrm{st}}=\mathrm{p}_{\infty}+1 / 2 \rho \mathrm{u}_{\infty}^{2}$ calculated from Bernoullı's equation For a shelter immersed in a boundary layer, the approach flow is modified by the presence of the ground This modification begins, according to surface-pressure measurements of Good and Joubert (1968) along a smooth floor at a distance of $\mathrm{x}=15(\delta / \mathrm{h})^{0}{ }^{7} \mathrm{~h}$ upstream of the shelter where $\delta$ is the boundary-layer thickness, upon approaching the shelter the flow is retarded more strongly near the surface than at higher elevations, and eventually separation occurs at a short distance in front of the shelter The separation "bubble" does not extend over the full height of the shelter, and a stagnation point exists at about $05 \mathrm{~h}$ to $07 \mathrm{~h}$ from the ground at the face of the shelter Below the stagnation streamline the flow is downward, a standing eddy forms in the corner between ground and shelter Above this eddy the flow is accelerating toward the upper edge of the shelter This flow field changes the pressure distribution from what would be expected had the ground not exerted any friction, in particular, the pressure maximum appears at the stagnation point and not at ground level

Clearly such a flow field does not exist in front of a porous shelter because the porosity does not permit a pressure buildup in front that leads to separation It is likely that at the rear of the screen the pressure is about constant, as it is for the solid-fence case, and that the velocity distribution across the screens is farly uniform The latter is evident in some of the velocity profiles reported by Blenk and Trienes (1956) A similar observation has also been made at the edge of a forest A low level jet is often observed which extends over the first few tree rows and then settles down to a roughly uniform velocity over a substantial part of the tree height (Reifsnyder, 1955, Meroney, 1967) This uniform velocity makes it likely that the pressure across the front of the shelters is also farly uniform, as long as the shelter is of approximately constant porosity In that case the pressure drop across the shelter is proportional to the square of the velocity through the shelter, with a factor of proportionality depending on the porosity only

The pressures in front and in the rear of the shelter are not independent because the alr flowing over the top of the shelter provides a "leak" connecting the two pressures However, the mechanism by which the pressure at the back side is governed is not yet understood The prediction of the base pressure is a problem of considerable importance in aerodynamics, and much work has been done on it, particularly for applications of supersonic aerodynamics (1 e, Brown and Stewartson, 1969, for a recent survey) For low-speed aerodynamics the problem apparently has parts that complicate its solution even further, such as the turbulence in the blending region which is likely to be an important factor in the transmission of pressure gradients, or 
the smaller pressure differences between base pressure and free-stream pressure, which leads to a curved separation streamline. The pressure field that arises is illustrated for the data of Chang (1966) in Fig. 4.10c, in which isobars have been indicated. It is noteworthy that substantial pressure gradients exist in both the longitudinal and vertical directions. These pressure gradients indicate that, in the sheltered region, it is not permissible to simplify the Navier-Stokes equations by using the boundary-layer assumptions.

Measurements along the ground have shown that the negative pressure downstream of the shelter is approximately constant over a distance of about three times the height $h$ and then starts rising very rapidly until, in the neighborhood of reattachment, it reaches a maximum that is slightly above the pressure in the free stream. Further downstream it slowly decreases and reaches asymptotically the free-stream pressure, as is required by the boundary-layer character of the redeveloping flow. This is illustrated in Fig. 4.17. The figure was obtained by plotting nondimensional pressure differences $\left(\mathrm{p}_{\mathrm{wall}}-\mathrm{p}_{\infty}\right) / \rho \mathrm{u}_{\infty}^{2}$ vs. $\mathrm{x} / \mathrm{h}$, and the distributions are independent of velocity and scale uniquely for a particular shelter

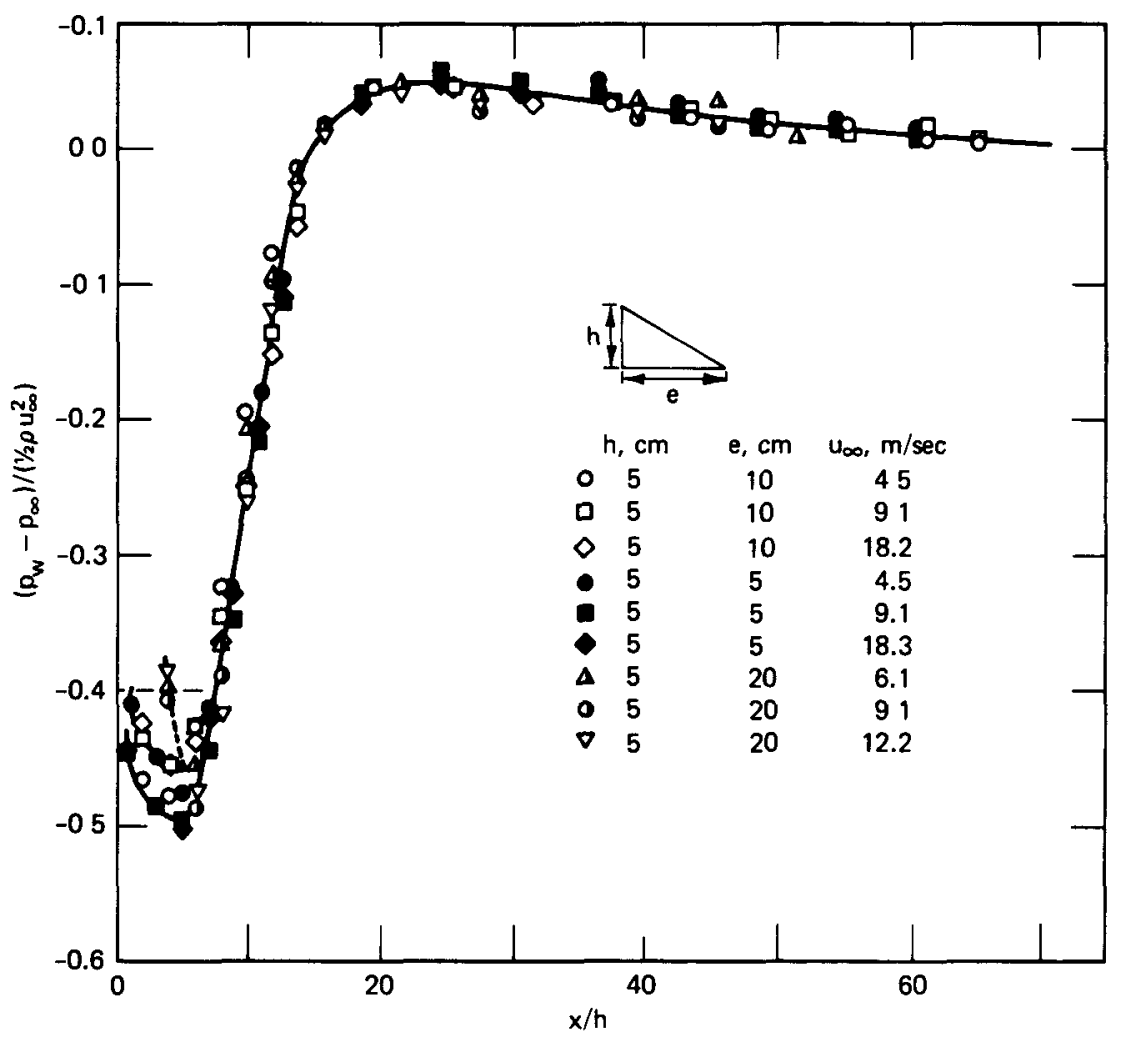

Fig. 4.17 Pressure distributions along the ground (from Plate and Lin, 1965) 
The difference between ground and free-stream pressure gives rise to a vertical force on the flow in the blending region which tends to push the separation streamline toward the ground. This is called the "Coanda" effect. The curvature of the streamlines, as expressed by the radius of curvature $\mathrm{R}$, can be approximately inferred by applying the momentum-conservation law to a small element out of the blending region, as indicated in Fig. 4.18.

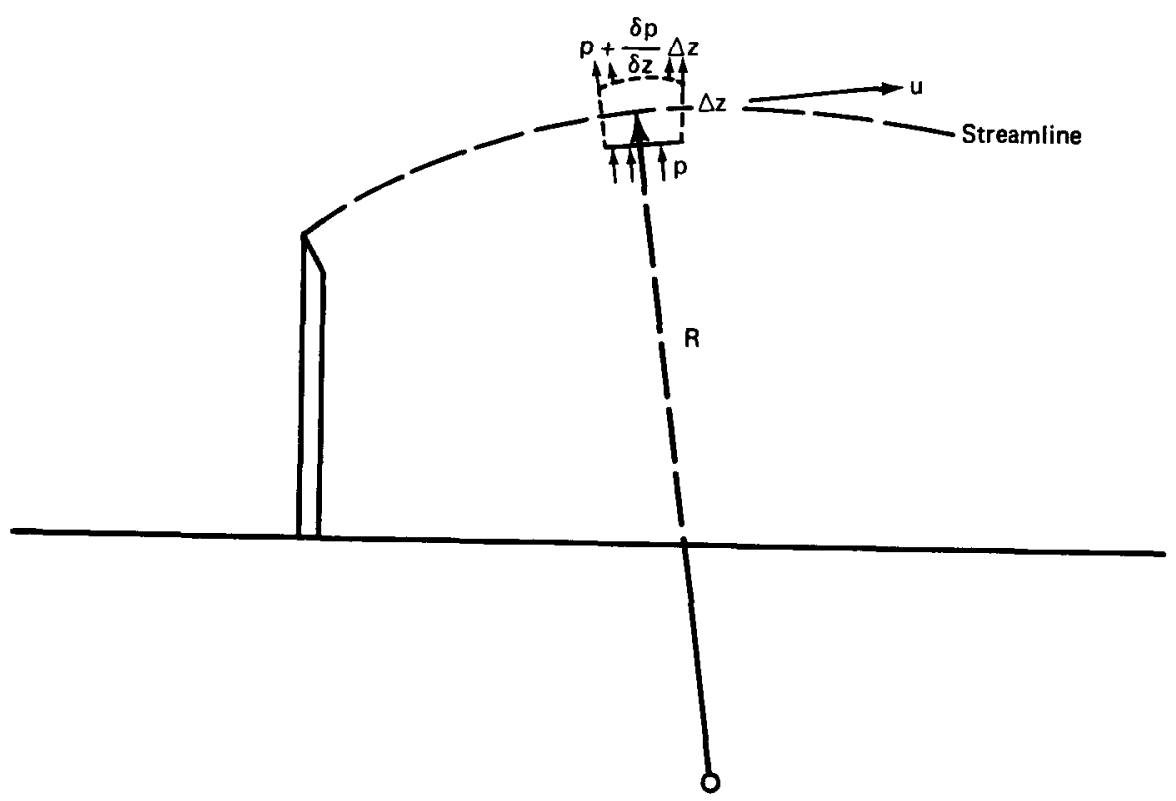

Fig. 4.18 Illustrating the Coanda effect.

Let the element have a velocity $u$, which, by the definition of a streamline, is directed normal to the radius of curvature $R$, and let the pressure across the element change by an amount $\partial \mathrm{p} / \partial \mathrm{z} \mathrm{dz}$. The centrifugal force on the element then must be balanced by the resultant of pressure forces and shear forces on the front and rear faces of the element; or, neglecting the latter, the momentum-balance equation yields

$$
\frac{1}{\mathrm{R}}=\frac{1}{\rho \mathrm{u}^{2}} \frac{\partial \mathrm{p}}{\partial \mathrm{z}}
$$

or in nondimensional form

$$
\frac{h}{R}=2 \frac{h}{x} \times \frac{p_{w a l l}-p_{\infty}}{1 / 2 \rho u_{\infty}^{2}} \times \frac{u_{\infty}^{2}}{u^{2}} \times \frac{\partial p^{\prime}}{\partial \eta}
$$


where

$$
\mathrm{p}^{\prime}=\frac{\mathrm{p}-\mathrm{p}_{\infty}}{\mathrm{p}_{\mathrm{wall}}-\mathrm{p}_{\infty}}=\mathrm{g}(\eta)
$$

The pressure-gradient function $\mathrm{g}(\eta)$ in Eq. 4.70 is probably depending on the geometry only and may be assumed to obey a similarity law depending on the same similarity variable $\eta$ as the mean velocity distribution. For the curve $u=0.5 u_{\infty}$, the function $2\left(\mathrm{u}_{\infty}^{2} / \mathrm{u}^{2}\right)\left(\partial \mathrm{p}^{\prime} / \partial \eta\right)$ is a constant $=\alpha$, and the radius of curvature becomes

$$
\frac{\mathrm{h}}{\mathrm{R}}=\alpha \frac{\mathrm{p}_{\mathrm{wall}}-\mathrm{p}_{\infty}}{1 / 2 \rho \mathrm{u}_{\infty}^{2}}
$$

with a maximum value of

$$
\frac{\mathrm{h}}{\mathrm{R}}=\alpha \mathrm{Q} \frac{\mathrm{h}}{\mathrm{X}}
$$

Although this equation represents only an approximation, it shows that the larger the pressure difference $p_{w a l l}-p_{\infty}$ the smaller the radius of curvature. A solid fence with a large base-pressure coefficient yields a more rapid turning of the $u=0.5 u_{\infty}$ curve toward the ground than a porous screen. The Coanda effect is at least in part responsible for the rapid loss of sheltering efficiency of a solid screen. A porous screen, with its higher velocity in the blending region and its lower base pressure, exhibits significantly smaller streamline curvature.

\section{Turbulence in the Sheltered Region}

More important than the Coanda effect on the wind field behind the shelter is the turbulence that is found in the air flow. The turbulence, whose vertical component is $\mathrm{w}^{\prime}$ and whose longitudinal component is $\mathrm{u}^{\prime}$, gives rise to turbulent shear stresses $\tau=-\rho \mathrm{u}^{\prime} \mathrm{w}^{\prime}$ that not only adjust the mean velocity distribution but are also responsible in producing turbulence. The strong mean velocity gradients generated by separation from the shelter edge interact with the shear stresses existing in the undisturbed flow to produce more turbulence for small values of $u_{b}$ than for large values. To see this, consider the energy balance of the turbulent motion, integrated over a volume consisting of a slice of (infinitesimal) length $\Delta x$, of infinite vertical extent and of unit width

$$
\Delta \mathrm{x} \int_{0}^{\infty} \frac{\mathrm{d \overline {q } ^ { 2 }}}{\mathrm{dt}} \mathrm{dz}=\Delta \mathrm{x} \int_{0}^{\infty} \frac{\tau}{\rho} \frac{\partial \overline{\mathrm{u}}}{\partial \mathrm{z}} \mathrm{dz}-\mathrm{D}
$$


where small terms have been neglected. The overbars denote time averages. In Eq. 4.73 $\mathrm{q}^{2}$ is the kinetic energy of the turbulent motion at a point

$$
\overline{q^{2}}=\frac{1}{2}\left(\overline{u^{\prime 2}}+\overline{v^{\prime 2}}+\overline{w^{\prime 2}}\right)
$$

and $D$ is the dissipation of kinetic energy into heat in the volume. The term on the left is the rate of change of turbulent energy in the volume which, for steady flows, can be expressed by

$$
\int_{0}^{\infty} \frac{d \overline{q^{2}}}{d t} d z=\frac{d}{d x} \int_{0}^{\infty} u q^{2} d z
$$

This term is determined mostly by the first term on the right in Eq. 4.73, which represents the turbulence production and which in the stages of growth of the separation layer considerably exceeds the dissipation. By inserting Eqs. 4.50 and 4.51 into the production term of Eq. 4.73, and by neglecting the dissipation term as compared with the production, we readily find that in the blending region the turbulence increases at a rate proportional to $\left(u_{\infty}^{2}-u_{b}^{2}\right)\left(u_{\infty}-u_{b}\right)$ and independent of $\mathrm{x}$, until the presence of the ground modifies the turbulence structure. Consequently a more solid fence has associated with it a larger turbulence level, i.e., the total amount of turbulence downstream of a porous screen is lower than that behind a solid screen. This situation will certainly affect the turbulent-transport processes in the sheltered region and will thus explain why higher evaporation rates are observed behind a solid screen than behind a porous screen, as was observed by Blenk and Trienes (1956). Perhaps a more important factor in determining exchange processes behind a solid screen is the return flow in the separation bubble which has associated with it strong vertical currents. These currents do not exist behind porous screens, so that for either of these reasons, if wind protection is the most important task of the shelterbelt, a porous screen has great advantages over a solid one.

\section{Application to Shelterbelt Design}

The preceding discussion indicates that sufficient information is not yet available for an aerodynamically most efficient shelterbelt design. But we now know what information is needed and how it can be obtained.

The first requirement is a knowledge of the velocity profile at the location of the shelter before the shelter is to be planted or constructed. This information is readily available; a good guess on the $u_{*}$ and $z_{0}$ values of the undisturbed boundary layer can be had either by taking some local measurements or by consulting Chap. 1 or the existing literature on the velocity distribution near natural surfaces, such as Geiger (1965, p. 275). But to go beyond this, we must make a number of assumptions that only for a solid shelterbelt are backed by experimental evidence. A drag coefficient 
and a base-pressure coefficient must be determined to predict the location of the separation streamline, and a velocity $u_{b}$ must be assumed Then it is possible to calculate the height $h^{*}$ from Eq 458 to a first approximation and to calculate the blending velocity profile by using Eq 452 , perhaps with $\sigma_{0}$ the solid-screen value

\section{RESEARCH NEEDS ON DISTURBED BOUNDARY LAYERS}

The two problems treated in this chapter are only a sample, albe1t an important one, of the types of problems that are encountered when real terrain effects must be considered Any added complexity of the terrain increases the difficulty of analytical treatment while at the same time making the situation more special and of less general applicability Very soon a point is reached when further investigations of more complex terrains become impractical, the amount of time and effort expended in solving the problem for the flow field is no longer in reasonable proportion to the value of the information obtained It is for these situations that field or wind-tunnel measurements on the actual terrain or a model thereof must be made There exist, however, a number of problems associated with aspects of disturbed boundary layers which are of sufficient generality to warrant further fundamental and analytical studies

A set of experiments must be made that exactly represents the analog of the analytical model of the internal boundary layer In this situation the turbulent energy balance should be determined to serve as a verification of the existing hypotheses (such as the shear-stress relation developed by Peterson, 1969) or as a starting point for improvements and modifications of existing models The modifications should include studies of the effect of zero-plane displacements (such as required for applying internal boundary-layer concepts to the flow field near the edge of a forest) and of finite lateral extent of the area of changed roughness Studies of interaction of internal boundary layers with the temperature field and with diffusing agents, as have already been done by Taylor (1970), need better experimental foundations and require laboratory investigations

All the internal boundary-layer models are concerned with an initially fully developed logarithmic profile, thus restricting their applicability to studies of the inner layer of the planetary boundary layer An investigation of the effect of a change of roughness on the geostrophic drag coefficient and on the outer part of the planetary boundary layer may yield useful information on how much area needs to be covered with uniform or approximately uniform roughness for the concept of a geostrophic drag coefficient to become valid The apparent success of inferring this coefficient from measured wind data over less than 1deal terrain, as shown in Fig 15 of Chap 1, lets it appear that such an area is much smaller than we would expect from internal boundary-layer calculations

To fill in the gaps in our knowledge of shelterbelt action, we should reanalyze existing wind-tunnel data and field data of flows behind shelterbelts and perform 
parallel experiments in wind tunnels and in the field. Natural shelter materials preclude the use of wind tunnels for the direct design of an optimum shelter. Since leaf area, growth rate, density, and shape of shelterbelts vary too much to be predictable in advance, the greatest use of a wind tunnel may be found in one of the two following areas.

The wind tunnel can be useful in evaluating the efficiency and arrangement of windbreaks made of nonrandom materials, which may consist of regular arrangements of slats, reeds, bamboo, or other material of a similar kind. These materials can be studied effectively in a wind tunnel because their geometry is reproducible, and their drag characteristics can be determined in the same manner as was described for screens. We can investigate in the same way the optimum design of a windbreak with respect to cost and efficiency. Some experiments by Japanese workers (Sato et al., 1952) along these lines have already been performed. During these experiments due regard must be given to the approach velocity profile, its roughness height $z_{0}$, and its shear velocity $\mathrm{u}_{*}$, as well as to the other aerodynamic factors of the surroundings which might affect the sheltering.

The second application is to the evaluation of the effect of terrain modifications in the neighborhood of the shelter on existing shelterbelts. Field experiments yield the necessary information on the approach wind distribution and on the sheltering from which a model of the undisturbed situation can be made. As was pointed out earlier, geometric similarity might suffice for this purpose in addition to scaling of the $z_{0}$ values by the scale of the shelter model. If the experiments yield significantly different sheltering coefficients, improvements can be made by trial and error by blocking or widening the interstices between the shelter elements.

Design of new shelters and classification of existing shelters may be aided by some fundamental research that would improve our understanding of the aerodynamic effects of shelterbelts. From the discussion in previous sections it is clear that the effect of porosity on the sheltering is incompletely understood, as is the effect of roughness on drag. To this we may add systematic investigations of the effect of finite-width shelters, shelters that are not oriented perpendicular to the wind, and the modification of the flow field by multiple shelterbelts.

\section{REFERENCES}

\section{Internal Boundary Layers}

Antonia, R. A., and R. E. Luxton, 1968, The Response of a Turbulent Boundary Layer to a Step Change in Surface Roughness, Preliminary Report, Department of Mechanical Engineering, University of Sydney.

Arago, L. R., 1959, On the Vertical Motions in the Atmosphere Above a Thermally Inhomogeneous Surface, Tr. Gl. Geofiz. Observ., 99: 112-121.

Blackadar, A. K., P. E. Glass, and H. A. Panofsky, 1967, A Theory of the Effects of Stability upon the Wind Profile Under Conditions of Inhomogeneous Terrain Roughness, in Proceedings of the 
USAEC Meteorological Information Meeting, Chalk River Nuclear Laboratories of Ontario, C A Mawson (Ed), Canadian Report AECL-2787, pp 453-461

Blackadar, A K, et al, 1967, Determination of the Effect of Roughness Change on the Wind Profile, in Boundary Layers and Turbulence, Phys Fludds Suppl 1967 209-211

Blom, J., and L Wartena, 1969, The Influence of Changes in Surface Roughness on the Development of the Turbulent Boundary Layer in the Lower Layers of the Atmosphere, $J$ Atmos Scl 26 255-265

Bradley, E F , 1968, A Micrometeorological Study of Velocity Profiles and Surface Drag in the Region Modified by a Change in Surface Roughness, Quart $J$ Roy Meteorol Soc 94 361-379

Bradshaw, P, 1969, Comments on On the Relation Between the Shear Stress and the Velocity Profile After a Change in Surface Roughness, $J$ Atmos Scl, 26: 1353-1354

Bradshaw, P, D H Ferriss, and N P Atwell, 1967, Calculation of Boundary-Layer Development Using the Turbulent Energy Equation, $J$ Fluld Mech $28 \quad 593-616$

Carson, J E, and D M Nelson, 1969, Chicago Aircraft Sounding Program, in Radiological Physics Division Annual Report, July 1968-June 1969, USAEC Report ANL-7615, pp 135-142, Argonne National Laboratory

Dimitriev, A A, and I N Sokolova, 1954 A Scheme for Evaluation of the Wind Velocity Changes in Alrflow, Passing over a Land-Sea Shore, Tr Hydrophys Institut IV: 87-93

Dyer, A J, 1963, Adjustment of Profiles and Eddy Fluxes, Quart $J$ Roy Meteorol Soc 89 276-280

Dyer, A J, and T V Crawford, 1965, Observations of the Modification of the Microclimate at a Leading Edge, Quart J Roy Meteorol Soc 97.345-348

Ellott, W P, 1958, The Growin of the Atmospheric Internal Boundary Layer, Trans Amer Geophys Unton 39 1048-1054

Estoque, M A , 1967, A Theoretical Investigation of the Sea Breeze, Quart J Roy Meteorol Soc 87: 136-146

Estoque, M A , 1962, A Sea Breeze as a Function of Prevalling Synoptic Situation, J Atmos Scl 19: $244-250$

Gandın, L S , 1952, On the Transtormation of the Wind Profile, $\operatorname{Tr}$ Gl Geofiz Observ 33 71-84

Jacobs, W, 1939, Unformung emes turbulenten Geschwindigkeitsprofiles, $Z$ Angew Math Mech 19 87-100 (Translation of National Advisory Committee for Aeronautics Technical Memorandum No 951)

Kung, R., 1970, Boundary-Layer Development over Equally Spaced Fences, unpublished Ph D Dissertation, Department of Civl Engineering, Colorado State Unıversity, Ft Collins, Colo

Kutzbach, J E, 1961, Investigations of the Modification of Wind Profiles by Artificially Controlled Surface Roughness, University of Wisconsin, Department of Meteorology, Annual Report, $p 71$

Logan, E, and J B Jones, 1963, Flow in a Pipe Following an Abrupt Increase in Surface Roughness, Trans ASME, (Amer Soc Mech Eng) Ser D, J Basic Eng, 85: 35-40

Meroney, R N, 1968, Characteristics of Wind and Turbulence in and Above Model Forests, $J$ Appl Meteorol , 7: 780-788

Moroz, W J , 1967, Lake Breeze on the Eastern Shore of Lake Michıgan Observation and Model, $J$ Atmos Scl, 24:337-355

Nadejdina, E D, 1964, On the Change of Meteorological Elements in Case of Aurflow Transformation, $\operatorname{Tr}$ Gl Geofiz Observ, 150 3-13

Nadejdina, E D, 1966, The Use of the Energy Balance Equation in the Problem for Auflow Transformation, $\operatorname{Tr}$ Gl Geofiz Observ, 187 69-76

Nickerson, E V , 1968, Boundary-Layer Adjustment as an Inıt1al Value Problem, J Atmos Scl 25: 207213

Onıshı, G, and M A Estoque, 1968, Numerical Study on Atmosphenc Boundary-Layer Flow over Inhomogeneous Terrain, J Meteorol Soc Jap 46 280-286 
Panchev, S, and N Godev, 1970, Wind Profile and Vertical Motions Above an Abrupt Change in Surface Roughness and Temperature, Department of Meteorology, Faculty of Physics, University of Sofia, Bulgana

Panofsky, H A, and A A Townsend, 1964, Change of Terrain Roughness and the Wind Profile, Quart J Roy Meteorol Soc, 90 147-155

Peterson, E W, 1969, Modification of Mean Flow and Turbulent Energy by Change in Surface Roughness Under Conditions of Neutral Stability, Quart J Roy Meteorol Soc, 90 561-576

Philip, J R, 1959, The Theory of Local Advection, J Meteorol, 16 535-547

Plate, E J , and G M. Hidy, 1967, Laboratory Study of Arr Flowing over a Smooth Surface onto Small Water Waves, $J$ Geophys Res $724627-4641$

Rider, N E, J R Philıp, and E F Bradley, 1963, The Horizontal Transport of Heat and Moisture - a Micrometeorological Study, Quart J Roy Meteorol Soc, 89 506-531

Schlichting, H, 1968, Boundary Layer Theory, 6th ed, McGraw-Hill Book Company, Inc, New York

Smith, F B , 1967, Modification of the Wind Profile Due to Changes in Surface Roughness, in Proceedings of the USAEC Meteorological Information Meeting, Chalk River Nuclear Laboratones, Sept 11-14, 1967, C A Mawson (Ed), Canadian Report AECL-2787, pp $463-475$

Stearns, C. R, and H H Lettau, 1963, Two Wind-Profile Measurement Experiments in Airflow over the Ice of Lake Mendota, Annual Report, p 115, Unıversity of Wisconsin, Department of Meteorology

Stearns, C R , 1964, Wind-Profile Modification Experiments Using Fields of Christmas Trees on the Ice of Lake Mendota, $l b l d, p$ 115, University of Wisconsin, Department of Meteorology

Tani, I , 1968, Review of Some Experimental Results on the Response of a Turbulent Boundary Layer to Sudden Perturbations, in AFOSR-IFP-Stanford Conference on Computations of Turbulent Boundary Layers, Vol 1, pp 483-494, S J Kline et al (Eds), Thermo Sciences Division, Department of Mechancal Engineering, Stanford Unıversity, Stanford, Cahf

Tanı, I, and H Makıta, 1968, Wind Tunnel Data, Department of Mechanical Engineerıng, Nihon University, Tokyo, unpublished

Taylor, R J, 1962, Small-Scale Advection and the Neutral Wind Profile, J Fluid Mech, 13 529-539

Taylor, P A, 1969a, On Wind and Shear-Stress Profiles Above a Change in Surface Roughness, Quart $J$ Roy Meteorol Soc, 95 77-91

Taylor, P A, 1969b, The Planetary Boundary Layer Above a Change in Surface Roughness, $I$ Atmos Scl, $26 \quad 432-440$

Taylor, P A, 1970, A Numerical Model of Airflow Above Changes in Surface Heat Flux, Temperature, and Roughness for Neutral and Unstable Conditions, to be published in $J$ Bound Layer Meteorol, 1: 18-39

Townsend, A A, 1956, The Structure of Turbulent Shear Flow, Cambridge University Press, $315 \mathrm{pp}$

Townsend, A. A, 1965, The Response of a Turbulent Boundary Layer to Abrupt Changes in Surface Conditions, $J$ Fluid Mech , 22: 799-892

Townsend, A A, 1966, The Flow in a Turbulent Boundary Layer After a Change in Surface Roughness, $J$ Fluid Mech , 26 255-266

Wagner, N K., 1966, A Two-Dimensional, Time Dependent Numerical Model of Atmospheric Boundary-Layer Flow over Inhomogeneous Terrain, in Theoretical Studies of the Atmospheric Boundary Layer, Final Report to U S Army Electronic Research and Development Activity, pp 1-80, Hawall Institute of Geophysics, University of Hawall

Yeh, F F, Dissertation

Zaitsev, A S , 1963, Transformation of the WInd Profile with the Change of Turbulence Intensity, $\operatorname{Tr} \mathrm{Gl}$ Geofiz Observ, 95 42-46. 


\section{Shelterbelts}

Arie, M , and H Rouse, 1956, Experıments on Two-Dimensional Flow over a Normal Wall, $J$ Fluıd Mech 1129141

Blenk, H, and H Trienes, 1956, Stroemungstechnische Beitraege zum Windschultz, in Grundlagen der Landtechnık Vol 8, Pt 1 and 2, VDI Verlag, Duesseldorf

Brown, S N, and K Stewartson, 1969, Lamınar Separation, Annu Rev Flutd Mech, 1: 45-72

Chang, S C, 1966, Velocity Distributions in the Separated Flow Behınd a Wedge-Shaped Model Hill, unpublished M S thesis, Colorado State University

Geiger, R, 1965, The Climate near the Ground, 4th ed, Harvard University Press

Good, M C , and P C Joubert, 1968, The Form Drag of Two-Dimensional Bluff-Plates Immersed 1n Turbulent Boundary Layers, $J$ Fluld Mech $31547-582$

Halitzky, J , 1968, Gas Diffusion Near Buildings, in Meteorology and Atomic Energy 1968 Chap 5 5, pp 221-255

Jensen, M , 1954, Shelter Effect Danish Technical Press, Copenhagen

Kaiser, H , 1959, Die Stromung an Windschutzstreıfen, Ber Deut Wetterdien 7(53)

Meroney, R N , 1968, Characteristics of Wind and Turbulence In and Above Model Forests, $J$ Appl Meteorol 7(5) 780-788

Mueller, T J, H H Korst, and W L Chow, 1964, On the Separation Reattachment and Redevelopment of Incompressible Turbulent Shear Flow, Trans ASME (Amer Soc Mech Eng ) Ser D, J Basic Eng 86 221-226

Nagabhushanaiah, H S, 1961, Separation Flow Downstream of a Plate Set Normal to a Plane Boundary, unpublished Ph D Dissertation, Colorado State University

Nagelı, W , 1941, Untersuchungen uber die Windverhaltnisse 1m Bereich von Windschutzstreifen, Mitt Schweiz Anst Forstl Versuchsw 23 221-276

Plate, E J , 1964, The Drag on a Smooth Flat Plate with a Fence Immersed in Its Turbulent Boundary Layer, in ASME 1964 Flulds Engineering Conference May 18-20, 1964, Philadelphia, $\mathrm{Pa}$, American Society of Mechanıcal Engineers

Plate, E J, and C Y Lin, 1965, The Velocity Field Downstream from a Two-Dimensional Model Hıll, Final Report, Part I, to U S Army Material Agency

Plate, E J , 1967, Diffusion from a Ground-Level Line Source into the Disturbed Boundary Layer Far Downstream from a Fence, Int $J$ Heat Mass Transfer, 10 181-194, Pergamon Press, London

Reifsnyder, W E , 1955, Wind Profiles in a Small Isolated Forest Stand, Forest Scl $1289-297$

Sabin, C M 1965, An Analytical and Experimental Study of the Plane, Incompressible, Turbulent Free Shear Layer with Arbitrary Velocity Ratio and Pressure Gradient, $J$ Basic Eng ASME Trans Ser D 87. 421428

Sato, K, M Tamachı, K Teranda, Y Watanabe, T Katoh, Y Sakanone, and M Iwasakı, 1952, Studies on Wind Breaks, Nippon Gakujutsu-Shiukoka1, Tokyo, 201 pages

Schlich ting, H , 1968, Boundary Layer Theory McGraw-Hill Book Company, Inc , New York

Schubauer, G B, W G Spangenberg, and P Klebanoff, 1950, Aerodynamics of Demping Screens National Advisory Committee for Aeronautics, Technical Note 2001, $16 \mathrm{pp}$

Townsend, A A, 1956, The Structure of Turbulent Shear Flow Cambndge University Press, New York

Uchida, S, and T Suzukı, 1968, On a Similar Solution for a Turbulent Half-Jet Along a Curved Streamline, J Flutd Mech, 33 379-398

van der Linde, J, 1962, Trees Outside the Forest, in Forest Influences, pp 141-208, Food and Agncultural Organization of the United Nations, Rome 
○ 


\section{AUTHOR INDEX}

Page numbers in italics represent primary points of reference to authors' works.

Albertson, M. L., 29, 47

Allen, L. H., 34, 35, 45

Antonio, R. A., 140, 178

Arago, L. R., 155, 178

Arie, M., 162, 169, 172, 181

Arya, S. P. S., $69,73-75,84,85,89,94$

Atwell, N. P., 155, 156, 179

Baines, W. D., 132, 133

Ball, F. K., 63, 94, 121, 125, 126, 131, 134, 135

Barad, M. L., 81, 94

Batchelor, G. K., 11, 45, 80, 94

Birkhoff, G., 168

Blackadar, A. K., 1, 10, 14, 16-18, 37, 45, $158,178,179$

Blenk, H., 162, 166, 171-172, 176, 181

Blom, J., 154, 155, 158, 179

Boussinesq, J., 54, 94

Bradley, E. F., 69, 73-75, 81, 82, 83, 84, 95,

$140,142,143,158,179,180$

Bradshaw, P., 155, 156, 179

Brown, S. N., 172, 181

Bryson, R. A., 88, 94

Bunker, A. F., 128, 134

Businger, J. A., 53, 65, 69, 73-75, 80-84, 95 , 132-134

Busse, F. H., 113, 134
Calder, K. L., 39, 45, 54, 80, 95

Carson, J. E., 159, 160, 179

Catton, I., 108, 111, 134

Cermak, J. E., 84, 95

Chalıkov, D. V., 81, 97

Chamberlain, A. C., 27, 28, 45

Chandrasekhar, S., 105, 106, 134

Chang, P. C., 24, 47

Chang, S. C., 162-164, 173, 181

Chow, W. L., 162, 181

Chuang, H., 84, 95

Cionco, R. M., 32, 34, 45

Clarke, R. H., 21, 45

Clauser, F. H., 14, 15, 23, 37, 46

Coantic, M., 65, 95

Counihan, C., 39, 46

Cramer, H. A., 73, 75, 84, 96

Crawford, R. V., 88, 95, 159, 179

Csanady, G. T., 1, 14, 16-18, 21, 46

Curne, I. G., 120, 134

Davenport, A. G., 39-41, 46

Davidson, B., 92, 96

Deacon, E. L., 27, 28, 29, 46, 84, 95

Deardorff, J., 10, 46, 111, 112, 114, 116, $124,127,132,134,135$

De Marra1s, G. A., 46

Dimitriev, A. A., 155,179 
Dropkın, D , 113, 134

Dutton, J A , 53, 55, 95

Dyer, A J , 21, 47, 86-88, 95, 96, 146 , 159,179

Elder, J W , 120, 121, 127, 133, 134

Ellott, W P , 65, 77, 87, 88, 95, 146, 179

Ellison, T H 71, 72, 73, 76, 8395

Estoque, M A , 144, 155, 159, 179

Ferris, D H $155,156,179$

Fichtl, G H , 53, 55, 95

Fleagle, R J , 53, 65, 95

Foster, T D , 116, 117, 119, 120, 133, 134

Gandın, L S , 155, 179

Gayevskaya, G N , 65, 95

Geiger, R, 162176,181

Glass, P E , 158, 178

Globe, S , 113, 134

Godev, N, 155, 158, 180

Goldstein, R J , 108, 134

Good, M C , 162, 169, 170, 172,181

Goody, R M , 65, 66, 67, 77, 95

Gowen, R A , 85, 95

Graham, D J , 108, 134

Halıtzky, J , 181

Hama, F H , 26, 46

Herring, J R 102, 109, 112-114, 121, 134

Hidy, G M , 24, 47, 144, 147, 148, 149, 150,180

Hinze, J , 24, 25, $46 \quad 86,95$

Hoeber, $\mathrm{H}, 16,46$

Holzman, B , 82, 95

Howard, L N , 113, 134

Hs1, G , 27, 28, 45, 46

Humphrey, H W, 130, 135

Iwasak1, M , 178, 181

Izumı, Y , 69, 73-75, 81-84, 95

Jacobs, W, 139, 179

Jensen, M , 166, 181

Johnson, O , 74, 46

Johnson, W B , 16, 46

Jones, J B , 140, 179

Joubert, P C , 162, 169, 170, 172, 181

Ka1ser, H , 162, I8I

Kao, S K , 80, 95

Kasansk1, A B , 16, 46

Katoh, T , 178, 181

Kestın, J , 74, 95

Klebanoff, P S , 21, 43, 46, 171, 181

Klug, W , 83, 95

Kondratiev, K Y , 65, 95

Korst, $\mathrm{H} \mathrm{H}, 162,181$

Kraichnan, R H . 113,134
Kung, E C , 27, 28, 46

Kung, R , 140, 179

Kutzbach, J E, 179

Lakhtman, D L , 16, 47 51, 93,95 97

Laufer, J , 25

Lemon, E R , 33, 34, 45, 46

Leovy, C B , 92, 95

Lettau, H H , 10, 16, 20, 29, 38, 44, 46 $92,96,140,180$

Lick, W, 120,134

Lilly, D K , 122, 124-125, 127, 129, 134

Lin, C W , 81, 84, 96

Lin, C Y , 160, 173, 181

Ling, S C , 29, 33, 47

Lloyd, A , 39, 46

Logan, E , 139, 179

Ludlam, F H , 128, 134135

Lumley, J L , 35, 46, 83, 96

Luxton, R E , 140, 178

Makıta, $\mathrm{H}, 140,180$

Malkus, J S , 128, 134

Malkus, M. V R , 9, 47, 109-111, 112, 113, 135

McVehil, G E , 84, 96

Meroney, R N , 140, 144, 145, 172, 179 , 181

Monin, A S , 16, 46, 47, 51, 80, 81, 96, 97

Montgomery, R B , 27, 47

Moroz, W J , 161, 179

Morton, B R, 129-131, 135

Mueller, T J , 162,181

Nadejdına, E D , 158, 179

Nagabhushanaiah, H S , 169, 181

Nagel, W , 161, 166, 167, 171, 181

Nath, J H , 27, 28, 45, 46

Nelson, D M , 159, 160, 179

Nickerson, E V , 155, 179

Obukhov, A M , 80, 81, 96

Ogura, Y , 54, 81, 96

Onishı, G , 144, 155, 179

Owen, P R , 90, 91, 93, 96

Paeschke, W , 27, 29, 30, 33, 47

Panchev, S , 155, 158, 180

Pandolfo, J P , 83, 96

Panofsky, H A , 35, 46, 83, 96, 151, 154, 158,178

Pellew, A , 106, 135

Pelton, W L , 29, 47

Peterson, E W , 156 157, 177,180

Philip, J R , 146. 180

Phillips, N W , 54, 96 
Plate, E., 24, 27, 29, 30, 32, 33, 45, 47, 69, $73-75,81,84,85,89,94,144,147-150$, $160,161,169,173,180,181$

Prandtl, L., 10, 13, 37, 47, 115, 135

Priestley, C H. B., 27, 47, 70, 83, 87, 93, 96, 99, 115, 131, 132,135

Proudman, J., 72, 73, 96

Qurasshı, A. A., 27, 29, 30, 32, 33, 45, 47

Rayleigh, Lord, 105, 108, 135

Record, F. A , 73, 75, 84, 96

Reichardt, H., 25, 168

Reifsnyder, W. E., 172, 181

Reynolds, W. C., 9, 47

Richardson, P. D , 74, 95

Rider, N. E., 81, 96, 180

Robinson, J., 120,135

Roll, H. U., 24, 47, 128, 135

Rossby, C. G., 27, 47

Rotta, J.C., 79, 96

Rouse, H., 130, 135, 162, 169, 172, 181

Rowland, W. R., 117, 135

Sabin, C. M., 165, 181

Sakanone, Y., 178,181

Sandborn, V.A., 22, 47

Sato, K, 178, 181

Sayre, W. W., 29, 47

Schlichting, H., 26, 37, 38, 44, 47, 71, 74, $96,129,135,146,164,165,180,181$

Schmıdt, W., 129, 135

Schubauer, G. B., 171, 181

Scorer, R. S., 128,134, 135

Seguin, B., 65, 95

Silveston, P. L., 108, 111, 113,135

Slotta, L. S., 23, 47

Smith, F. B., 158, 180

Smith, J. W., 85, 95

Sokolova, I. N., 155, 179

Southwell, R. V., 106, 135

Spangenberg, W. G., 117, 135, 171, 181

Spregel, E. A., 54, 56, 66, 96, 110, 135

Stearns, C. R., 140, 180

Stewartson, K., 172, 181

Stoller, T., 33, 47

Suzukı, T., 164, 181

Swinbank, W. C., 2, 19-21, 47, 81, 83, 88, 96
Tamachı, M , 178, 181

Tan, H. S., 29, 33, 47

Tanı, I., 140, 180

Tanner, C. B., 29, 47

Taylor, G. I., 70, 96, 129-131, 135

Taylor, P. A., 151-153, 155, 158, 177, 180

Taylor, R. J., 81, 96, 153, 180

Telford, J. W., 121, 122, 128, 131, 132,

Tennekes, H., 1, 14, 15, 45

Teranda, K., 178, 181

Thomas, D. B., 111, 116, 135

Thomson, W. R., 90, 91, 93, 96

Thorpe, S. A., 70, 96

Tiederman, W. G., 9, 47

Townsend, A. A., 22, 47, 50, 65, 66, 71, 77, $97,111,116,135,151,153,154,156,158$, 180,181

Trienes, H., 162, 166, 171, 172, 176, 181

Turner, J. S., 72, 73, 76, 95, 128, 129, 132, 135

Uchida, S., 164,181

Uchıima, Z., 32, 34, 47

Van der Linde, J., 162, 181

Van Dyke, M , 15, 47

Vehrenkamp, J. E., 92, 97

Veron1s, G., 54, 56, 96, 111, 135

Wagner, N. K , 144, 155, 180

Warner, J , 121, 122, 128, 132, 135

Wartena, L., 154, 155, 158, 179

Watanabe, Y., 178, 181

Webb, E. K , 50, 81, 84, 97

Webster, C A. G., 77, 97

We1ler, H. S., 47

Wesseling, P., 113, 135

Wieghardt, K., 22, 47

Willis, G. E., 111-114, 116, 124, 134, 135

Wood, J. D., 76, 97

Wright, J. L , 32, 34, 35, 47

Wyngaard, C. J., 69, 73-75, 81-83, 84, 95

Yakushevskaya, K. E., 65, 95

Yamamoto, G., 83, 97

Yeh, F. F., 144, 180

Ylh, C. S., 130,135

Yokum, C. S., 34, 35, 45

Zartsev, A. S., 180

Zilıtınkevıch, S. S., 16, 47, 51, 81, 97 
- 


\section{SUBJECT INDEX}

\author{
Absorption coefficient, 66 \\ Absolute humidity, 62 \\ Adrabatıc lapse rate, 54 \\ Atmospheric boundary layer \\ (see Planetary boundary layer) \\ Atmospheric stability, definition of, 54 \\ Atmosphenc surface layer \\ (see Planetary boundary layer) \\ Backward facing step, 144 \\ Base pressure, 171 \\ Base-pressure coefficient, 167, 175-176 \\ Bernoull's equation, 39 \\ Blending region, of internal boundary \\ layers, 139, 151 \\ simildrity profile for, 153 \\ width of, 151 \\ Boundary-layer equations, 141 \\ Boundary layers, artificially \\ thickened, 39 \\ Boussinesq approximation, 55 \\ Bowen ratio, 64 \\ Brunt-Vaisala frequency, 131 \\ Buoyancy, effect on turbulence, 66 \\ Buoyancy force, 128 \\ Buoyant vortex, 128 \\ Canopy flow, definition of, 31
}

Centrifugal force, 174

Charactertstic length,

for dissipation, 156

of eddy viscosity, 37

of free convection, 103

of turbulence in planetary boundary

layer, 37

of unsteady free convection, 120

Characteristic time, of boundary layer adjustment, 5

of free convection, 103

of turbulent decay, 71, 156

Characteristic temperature, for free convection, 103

Characteristic velocity, of eddy

viscosity, 37

Circulation, 128

Coanda effect, 174-175

Conductive sublayer, 120

Conservation of energy, 52

of constant-thickness layers, 7

effect of humidity on, 63

in free convection, $100,122,125$

for internal boundary layers, 177

for stratıfied flow, 60,83

of turbulence, 71

Conservation of mass, 4

for free convection, 100

for internal boundary layers, 146 
for sheltered region, 168

for thermal plume, 130

for turbulent flow, 4

Conservation of momentum, for constant-thıckness laver, 7, 11

for free convection, 102-103

integral equation of Kàrman, 41

for internal boundary layers, 146, 176

for sheltered region, 167

for thermal plume, 130

for turbulent flow, 4,5

Conservation of temperature,

fluctuations, 60,66

Constant stress layer, 29, 72

Convection, downdraft between, 131

Convection layer, development of, 49,50

thickness of, 124

Coriolis acceleration, definition of, 2, 3

role in energy equation, 8

Critical-flux Richardson number, 51, 70, 72,76

effect of radiation on, 77

effect of stability on, 76

Critical gradient Richardson number, 51,76

Critical Rayleigh number, 106-108

Critical Reynolds number, 94

Critical time in unsteady free convection, 120

Critical wavelength, for onset of free convection, 108

Diffusion, of energy, 156

Diffusion coefficient, of energy, 156

Displacement thickness, 41

Dissipation of energy (see Energy dissipation)

Dissipation of temperature fluctuations, 132

Disturbed boundary layers, definition of, 99

Drag coefficient, of crops, 32 geostrophıc, 16, 80, 177 of shelterbelts, 168-171

Eddy diffusivity, 36, 43, 156-157, 164 definition of, 36 in stratufied flow, 69, 93 structure of, 36

Eddy viscosity (see Eddy diffusivity)

Ekman layer, in laminar flow, 11 in stratified flow, 93 in turbulent flow, 12, 14

Energy diffusion, 8, 68, 156

Energy dissipation, definition of, 8 in disturbed boundary layers, 156 maximum of, 9

in sheltered region, 176

in stratıfied flow, 59, 68, 78

Energy equation (see Conservation of energy)

Entrainment, in thermals, 130

Entrainment coefficient, 130

Equation of continuity (see Conservation of mass)

Equation of motion (see Conservation of momentum)

Equation of state, 52, 53

Error function, 164-165

Euler number, 6

Evaporation, 138, 176

Flow zones, 161

Flux Richardson number, 68 definition of, 68

in logarithmic layer, 68

Forced-convection layer, 49

Free-convection layer, 49, 70, 93, $99 \mathrm{ff}$

Free turbulent shear flow, 129, 166

Geostrophic wind, 4, 11

Geostrophıc drag coefficient (see Drag coefficient, geostrophic)

Gradient Richardson number, critical, 70,71

definition of, 69

Heat-conduction equation, 117

Heat equation (see Conservation of energy)

Heat flux, in steady conduction, 106

Heat flux, turbulent, countergradient, 132

definition of, 63

at ground, 90

height dependency of, 79

space average of, 123

time dependency of, 127

Heat transfer in free convection, Malkus' model, 109

Herring's model, 112

Humidity fluctuations, 61 
Inner layer, of internal boundary

layer, 139

of planetary boundary layer, $12 \mathrm{ff} ., 177$

of shelterbelt flow, 161

Intermittency, 22

Internal boundary layer, definition of, 137

edge of, 146-147, 155

in stratified flow, 158-159

thickness of, 139

Inversion, 50, 94, 121, 159

Kàrmàn's constant, 16, 23, 68, 157

Kàrmàn-Pohlhausen method, 146

KEYPS equation, 83

Lake Mendota, $W_{1 s}, 140$

Lake Michigan, 159

Latent heat flux, 63

Leaf area, 34

Local isotropy, 141

Logarithmic law, derivation of, 15

in internal boundary layers, $140,147,154$

near smooth wall, 23

in stratified flow, 68

over water, 24

wind-tunnel modelıng of, 44

Matched expansions, method of, 15

Mean temperature distribution, in

free-convection region, 131,132

in internal boundary layers, 158

in steady conduction, 104

in stratıfied flow, $85 \mathrm{ff}$

Memory, of boundary layers, 137

Mixing length, defintion of, 36-37

in free convection, 115

in internal boundary layers, 151,153155

in stratified flow, 80

Molecular-conduction layer, 50

Momentum thickness, 41

Monın-Obukhov length, $68 \mathrm{ff}$.

Nusselt number, definition of, 104

in free convection, $109,111,129$

Optımum porosity, 166

Optımum shelter, 161, 177-178

Outer layer, of internal boundary

layer, 139

of planetary boundary layer, $17 \mathrm{ff}, 177$
Planck function, 66

Planetary boundary layer, characteristic length of, 37

inner layer of, 12

outer layer of, $17 \mathrm{ff}$

sublayers of, 12

thickness of, 21

wind-tunnel modeling of, 38

Potential temperature, 53, 54, 101

Porous shelter, 162, 167, 171

Prandtl number, 90, 103, 109, 113

Pressure distribution, in sheltered region, 167 near surface discontinuity, 141

Pressure fluctuations, in free convection, 125 in stratified flow, 56,68

Pressure gradient, in separated region, 172

Radiation, 65, 122, 127

Ratio $\mathrm{K}_{\mathrm{H}} / \mathrm{K}_{\mathrm{M}}$, critical value of, 72 in stable flow, 85

in unstable flow, 83

Rayleigh number, definition of, 103, 106, 108

in steady free convection, 109-114

in unsteady free convection, 117, 121

Reattachment, 160,173

Relaminarizatıon, 51, 133

Reynolds number, 6

definition of, 6

for rough surfaces, 27,90

Reynolds analogy, 86, 91

Rossby number, definition of, 6 geostrophic, 16

for a surface, 15

Roughness discontinuity, 138, 141

Roughness height, for canopy flow, 27 , definition of, 14,22

in stratıfied flow, 80

Sea breeze, 159

Separation, on shelterbelts, 162, 172

on trees, 144

Separation bubble, 161-162

Separation streamlıne, 162, 166-167

Shear-stress distribution, in boundary

layers, 39

in canopy flow, 32

near ground, 35

in planetary boundary layer, 20

across separation streamlıne, 166 
Shear temperature, 86

Sheltered region, height of, 169

Simularity theory, of Monin and

Obukhov, 80

Small perturbations, in free convection, 104,106

Stability, in free convection, 105 index of Businger, 80 of stratıfied flow, 71 in unsteady free convection, 117

Stagnation pressure, 172

Steady convection, 100

Stanton number, definition of, 90 in stratıfied flow, 90-91

Stratified planetary boundary layer, stability of, 70 structure of, 49.51

Streamline, curvature of, 174 displacement of, 140

Sublayer, of tree-convection layer 113 viscous, 23

Superddiabatic layer, $50 \quad 88-89,124 \quad 127$

Temperature distribution, 86,115

Temperature gradient, nondimensional 86 tt 159

Thermals, $50,88,120-121,127-128$

Thermal plumes, $50,88,131$

in stable stratification, 3,22

Time average, detinition of, 4

Terran, unstormity of , 5,177

Turbulence production, $8,144,176$

by buoyancy, $60,64,68$
Turbulent kınetıc energy, 8

Turbulent self-interaction, 102

Velocity distributions, in free convection, 130

near the ground, 13

for internal boundary layers, 147, $148 \mathrm{ft}$

in laminar Ekman layers, 11

loganithmic (see Logarithmic law)

power law, 38

behind shelterbelts, 164

in stratıfied boundary layers, $51,78 \mathrm{ff}$, 159

in thermals, 130

in viscous sublayer, 23

Velocity gradient, $79,80,83,159$

Virtual ongin, 165

Virtual potential temperature, 62

Viscous sublayer, thickness of, 23

Water vapor, 61

Wavelength, of instablity in steady free convection, 108

of instability in unsteady free convection, 117

Windless convection, 99, 106, 116

Wind-tunnel modeling, of internal boundary layers, 162, 178

of logarithmic layer, 44

of planetary boundary layer, 38

Zero-plane displacement, 27, 29, 177

\section{NOTICE}

This book was prepared under the sponsorship of the United States Government Neither the United States nor the United States Atomic Energy Commission nor any of their employees nor any of their contractors subcontractors, or their emplovees makes any warranty express or implied or assumes any legal liability or responsibility for the accuracy, completeness or usefulness of any information apparatus product or process disclosed or represents that its use would not infringe privately owned rights 